\title{
IUPAC-NIST Solubility Data Series. 98. Solubility of Polycyclic Aromatic Hydrocarbons in Pure and Organic Solvent Mixtures: Revised and Updated. Part 2. Ternary Solvent Mixtures
}

\author{
William E. Acree, Jr. ${ }^{\text {a) }}$ \\ Department of Chemistry, University of North Texas, Denton, Texas 76203, USA
}

(Received 13 November 2012; accepted 28 November 2012; published online 28 February 2013)

\begin{abstract}
This work updates Vols. 54, 58, and 59 in the IUPAC Solubility Data Series and presents solubility data for polycyclic aromatic hydrocarbon solutes dissolved in ternary organic solvent mixtures. Published solubility data for anthracene, phenanthrene, and pyrene that appeared in the primary literature between 1995 to the end of 2011 are compiled and critically evaluated. Experimental solubility data for 119 different solute-ternary solvent systems are included in the volume. Solubility data published prior to 1995 were contained in three earlier volumes (Vols. 54, 58, and 59) and are not repeated here. (C) 2013 American Institute of Physics. [http://dx.doi.org/10.1063/1.4773073]
\end{abstract}

Key words: alcohols; alkanes; alkoxyalcohols; anthracene; dialkyl ethers; phenanthrene; polycyclic aromatic hydrocarbons; pyrene; solubility; ternary solvents.

\section{CONTENTS}

1.

$\begin{array}{ll}\text { Preface } \ldots \ldots \ldots \ldots \ldots \ldots \ldots \ldots \ldots \ldots \ldots & 2 \\ 1.1 . & \text { Scope of this volume } \ldots \ldots \ldots \ldots \ldots \ldots \ldots \ldots\end{array}$

1.2. Procedures used in critical evaluation of published solubility data ..............

2. Solubility of Anthracene in Ternary Alkane + Alkane + Alcohol Solvent Mixtures..........

2.1. Critical evaluation of experimental solubility data.

2.2. Anthracene solubility data in ternary heptane + cyclohexane + alcohol solvent mixtures .

2.3. Anthracene solubility data in ternary $2,2,4-$ trimethylpentane + cyclohexane + alcohol solvent mixtures......................

3. Solubility of Anthracene in Ternary Alkane + Alcohol + Alcohol Solvent Mixtures .........

3.1. Critical evaluation of experimental solubility data.............................

3.2. Anthracene solubility data in ternary heptane + alcohol + alcohol solvent mixtures .....

3.3. Anthracene solubility data in ternary cyclohexane + alcohol + alcohol solvent mixtures ...........................

3.4. Anthracene solubility data in ternary $2,2,4-$ trimethylpentane + alcohol + alcohol solvent mixtures
4. Solubility of Anthracene in Ternary Alkane + Ether + Alcohol Solvent Mixtures ...........

4.1. Critical evaluation of experimental solubility data ......................

4.2. Anthracene solubility data in ternary alkane + $1,1^{\prime}$-oxybisbutane + alcohol solvent mixtures

\section{2}

3

4.3. Anthracene solubility data in ternary alkane + 2-methoxy-2-methylpropane + alcohol solvent mixtures.....................

4.4. Anthracene solubility data in ternary alkane + 1,4-dioxane + alcohol solvent mixtures....

5. Solubility of Anthracene in Ternary Alkane + Alkane + Alkoxyalcohol Solvent Mixtures .....

5.1. Critical evaluation of experimental solubility data $\ldots \ldots \ldots \ldots \ldots \ldots \ldots$

5.2. Anthracene solubility data in ternary alkane + alkane +2 -ethoxyethanol solvent mixtures

5.3. Anthracene solubility data in ternary alkane + alkane +2 -butoxyethanol solvent mixtures

6. Solubility of Anthracene in Ternary Alkane + Alcohol + Alkoxyalcohol Solvent Mixtures ....

6.1. Critical evaluation of experimental solubility data .....................

6.2. Anthracene solubility data in ternary alkane + alcohol + 2-butoxyethanol solvent mixtures

7. Solubility of Anthracene in Ternary Alcohol + Alcohol + Alkoxyalcohol Solvent Mixtures ....

7.1. Critical evaluation of experimental solubility data......................

7.2. Anthracene solubility data in ternary alcohol + alcohol + 2-butoxyethanol solvent mixtures

\footnotetext{
a)Electronic mail: acree@unt.edu.

(c) 2013 American Institute of Physics.
} 
8. Solubility of Anthracene in Ternary Alkane + Alkanone + Alcohol Solvent Mixtures. .......

8.1. Critical evaluation of experimental solubility data....................

8.2. Anthracene solubility data in ternary $2,2,4-$ trimethylpentane + propanone + alcohol solvent mixtures...................

9. Solubility of Anthracene in Miscellaneous Ternary Solvent Mixtures ................

9.1. Critical evaluation of experimental

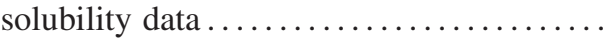

9.2. Anthracene solubility data in ternary cyclohexanone + ethyl ethanoate + methanol solvent mixtures....................

10. Solubility of Phenanthrene in Ternary Alcohol + Alcohol + Alcohol Solvent Mixtures ........

10.1. Critical evaluation of experimental solubility data

10.2. Phenanthrene solubility data in ternary alcohol + alcohol + alcohol solvent mixtures

11. Solubility of Pyrene in Ternary Alkane + Alkane + Alcohol Solvent Mixtures..............

11.1. Critical evaluation of experimental solubility data....................

11.2. Pyrene solubility data in ternary heptane + cyclohexane + alcohol solvent mixtures. .

12. Solubility of Pyrene in Ternary Alkane + Alcohol

+ Alcohol Solvent Mixtures ............

12.1. Critical evaluation of experimental

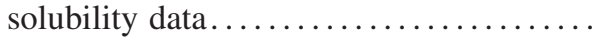

12.2. Pyrene solubility data in ternary heptane + alcohol + alcohol solvent mixtures......

12.3. Pyrene solubility data in ternary cyclohexane + alcohol + alcohol solvent mixtures ..........................

12.4. Pyrene solubility data in ternary $2,2,4-$ trimethylpentane + alcohol + alcohol solvent mixtures ....................

13.

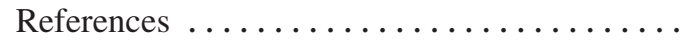

\section{List of Tables}

1. Combined NIBS/Redlich-Kister $S_{23, i}$ parameters for mathematical representation of anthracene mole fraction solubilities in binary solvent mixtures ...............................

2. Combined NIBS/Redlich-Kister $S_{23, i}$ parameters for mathematical representation of pyrene mole fraction solubilities in binary solvent

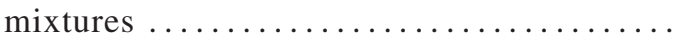

3. Summarized comparison between experimental solubilities of anthracene in ternary alkane + alkane + alcohol solvent mixtures and predicted values based on Eq. (2) .................

4. Summarized comparison between experimental solubilities of anthracene in ternary alkane + alcohol + alcohol solvent mixtures and predicted values based on Eq. (2)

5. Summarized comparison between experimental solubilities of anthracene in ternary alkane + ether + alcohol solvent mixtures and predicted values based on Eq. (2) ....................

6. Summarized comparison between experimental solubilities of anthracene in ternary alkane + alkane + alkoxyalcohol solvent mixtures and predicted values based on Eq. (2).............

7. Summarized comparison between experimental solubilities of anthracene in ternary alkane + alcohol + alkoxyalcohol solvent mixtures and predicted values based on Eq. (2)............

8. Summarized comparison between experimental solubilities of anthracene in ternary alcohol + alcohol + alkoxyalcohol solvent mixtures and predicted values based on Eq. (2)............

9. Combined NIBS/Redlich-Kister $S_{23, i}$ parameters for mathematical representation of phenanthrene molar solubilities in binary solvent mixtures ...

10. Summarized comparison between experimental solubilities of phenanthrene in ternary alcohol + alcohol + alcohol solvent mixtures and predicted values based on Eq. (5) .................

11. Summarized comparison between experimental solubilities of pyrene in ternary alkane + alkane + alcohol solvent mixtures and predicted values based on Eq. (2) .....................

12. Summarized comparison between experimental solubilities of pyrene in ternary alkane + alcohol + alcohol solvent mixtures and predicted values based on Eq. (2) .......................

\section{Preface}

\subsection{Scope of this volume}

Volumes $54,{ }^{1} 58,{ }^{2}$ and 59 (Ref. 3) in the IUPAC Solubility Data Series dealt with the solubility of polycyclic aromatic hydrocarbons (PAHs) and polycyclic aromatic hetero-atom compounds (PAHaCs) in both neat organic solvents and binary organic solvents. The specific solutes considered included: acenaphthene, acridine, anthracene, benz [a]anthracene, benzo [b]fluorene, benzo[ghi]perylene, benzo[a]pyrene, biphenyl, 2,2'-bipyridine, buckminsterfullerene (C60), carbazole, chrysene, coronene, dibenz[a,h]anthracene, dibenzofuran, dibenzothiophene, 1,2-diphenylbenzene, 1,3-diphenylbenzene, 1,4diphenylbenzene, fluoranthene, fluorene, indole, naphthacene, naphthalene, perylene, phenanthrene, 1,10-phenanthroline, phenothiazine, phenoxanthiin, phenoxazine, pyrene, thianthrene, thioxanthene, triphenylene, and xanthenes. The three volumes covered the published literature up to 1995, including 11 several articles that were in still in press at the time. 
This paper is the second part of a three-part series that will update the three earlier volumes on PAH and PAHaC solubilities. To conserve space, data from the earlier volumes will not be repeated here. Part 1 [Ref. 4] was devoted to solubilities in binary solvent mixtures, and incorporates compilations based on papers published in the peer-reviewed scientific literature between 1995 and the end of 2011. Part 2 (this paper) will deal with solubilities of PAHs and PAHaCs dissolved in ternary solvent mixtures, while Part 3 will focus on the organic monosolvents (neat organic solvents).

\subsection{Procedures used in critical evaluation of published solubility data}

The different concentration units that are used to express experimental solubility data, and the thermodynamic principles that govern solubility solid-liquid equilibria, were described in Part 1 of this volume. ${ }^{4}$ The only part of the earlier discussion that will be repeated here pertains to the mathematical representation of solubility data for crystalline nonelectrolyte solutes dissolved in binary solvent mixtures, and the use of the calculated curve-fit coefficients to predict the solute's solubility in ternary and higher order multicomponent solvent mixtures.

Procedures used in the critical evaluation of published solubility data for crystalline nonelectrolytes dissolved in organic monosolvents and organic solvent mixtures depend to a large extent on the quantity and type of data to be evaluated. In those instances where independent experimental measurements exist, one can compute the mean value and standard deviation for each set of replicate values (or set of values). This type of analysis will be limited primarily to the neat mono-solvents, as published data for binary and ternary solvent mixtures are relatively scarce compared to solubility data for solutes dissolved in single solvent systems. Researchers have tended to perform measurements on new mixtures as opposed to repeating measurements on already studied mixtures, even if measured at different temperatures.

The solvent composition dependence of solubility is generally evaluated using semi-theoretical solution models. Of the theoretical solution models developed over the last 50 years, the Combined Nearly Ideal Binary Solvent (NIBS)/RedlichKister equation

$$
\begin{aligned}
\ln x_{1}{ }^{\mathrm{sat}}= & x_{2}{ }^{(\mathrm{s})} \ln \left(x_{1}{ }^{\mathrm{sat}}\right)_{2}+x_{3}{ }^{(\mathrm{s})} \ln \left(x_{1}{ }^{\mathrm{sat}}\right)_{3} \\
& +x_{2}{ }^{(\mathrm{s})} x_{3}{ }^{(\mathrm{s})} \sum_{j=0}^{r} S_{23, j}\left(x_{2}{ }^{(\mathrm{s})}-x_{3}{ }^{(\mathrm{s})}\right)^{j}
\end{aligned}
$$

is ${ }^{5,6}$ likely the most popular of the proposed mathematical representations. In the above equation, $x_{i}{ }^{(\mathrm{s})}$ refers to the initial mole fraction solvent composition of component $i$ calculated as if the solute were not present, and $\left(x_{1}{ }^{\text {sat }}\right)_{i}$ denotes the measured solute solubility in pure solvent $i$. The summation in the last term on the right-hand side of Eq. (1) includes as many curve-fit $S_{23, i}$ parameters as are needed to accurately describe the observed solubility data. Generally, no more than three parameters will be needed in a given mathematical representation. The various $S_{23, i}$ parameters are determined by regression analysis. Numerical values of $S_{23, i}$ parameters used in the mathematical representation of the mole fraction solubility of anthracene and pyrene in selected binary solvent systems are compiled in Tables 1 and 2, respectively. More extensive compilations can be found in Part 1 of this volume 4 and in Vols. 58 (Ref. 2) and 59 (Ref. 3) of the IUPAC Solubility Data Series.

TABLE 1. Combined NIBS/Redlich-Kister $S_{23, i}$ parameters for mathematical representation of anthracene mole fraction solubilities in binary solvent

\begin{tabular}{|c|c|c|}
\hline Solvent (2) + solvent (3) & $S_{23, i}{ }^{\mathrm{a}}$ & $\% \operatorname{Dev}^{b}$ \\
\hline \multicolumn{3}{|l|}{ Alkane + alkane binary mixtures } \\
\hline Heptane (2) + cyclohexane (3) & $\begin{array}{r}0.153 \\
-0.089 \\
-0.084\end{array}$ & 1.3 \\
\hline Cyclohexane (2) $+2,2,4$-trimethylpentane (3) & $\begin{array}{l}-0.011 \\
-0.021\end{array}$ & 0.2 \\
\hline \multicolumn{3}{|l|}{ Alkane + alcohol binary mixtures } \\
\hline Heptane (2) + 1-propanol (3) & $\begin{array}{r}1.098 \\
-0.106 \\
0.324\end{array}$ & 0.4 \\
\hline Cyclohexane (2) + 1-propanol (3) & $\begin{array}{l}1.121 \\
0.040 \\
0.256\end{array}$ & 0.5 \\
\hline 2,2,4-Trimethylpentane (2) + 1-propanol (3) & $\begin{array}{r}0.825 \\
-0.103 \\
0.291\end{array}$ & 0.4 \\
\hline Heptane (2) + 2-propanol (3) & $\begin{array}{r}1.491 \\
-0.587 \\
0.566\end{array}$ & 0.3 \\
\hline Cyclohexane (2) + 2-propanol (3) & $\begin{array}{r}1.589 \\
-0.143 \\
0.248\end{array}$ & 0.3 \\
\hline 2,2,4-Trimethylpentane (2) + 2-propanol (3) & $\begin{array}{r}1.193 \\
-0.369 \\
0.333\end{array}$ & 0.3 \\
\hline Heptane (2) + 1-butanol (3) & $\begin{array}{l}0.723 \\
0.004 \\
0.201\end{array}$ & 0.2 \\
\hline Cyclohexane (2) + 1-butanol (3) & $\begin{array}{l}0.741 \\
0.345 \\
0.223\end{array}$ & 0.5 \\
\hline 2,2,4-Trimethylpentane (2) + 1-butanol (3) & $\begin{array}{l}0.536 \\
0.151 \\
0.142\end{array}$ & 0.3 \\
\hline Heptane (2) + 2-butanol (3) & $\begin{array}{r}1.225 \\
-0.292\end{array}$ & 0.2 \\
\hline Cyclohexane (2) + 2-Butanol (3) & $\begin{array}{l}1.260 \\
0.206\end{array}$ & 0.6 \\
\hline 2,2,4-Trimethylpentane (2) + 2-butanol (3) & $\begin{array}{r}1.070 \\
-0.213\end{array}$ & 0.8 \\
\hline Heptane (2) + 2-methyl-1-propanol (3) & $\begin{array}{r}1.284 \\
-0.301 \\
0.266\end{array}$ & 0.7 \\
\hline Cyclohexane (2) + 2-methyl-1-propanol (3) & $\begin{array}{l}1.116 \\
0.172 \\
0.341\end{array}$ & 0.3 \\
\hline 2,2,4-Trimethylpentane (2) + 2-methyl-1-propanol (3) & $\begin{array}{r}0.972 \\
-0.100 \\
0.462\end{array}$ & 0.4 \\
\hline Cyclohexane (2) + 1-pentanol (3) & $\begin{array}{l}0.591 \\
0.472 \\
0.010\end{array}$ & 0.7 \\
\hline
\end{tabular}
mixtures 
TABLE 1. Combined NIBS/Redlich-Kister $S_{23, i}$ parameters for mathematical representation of anthracene mole fraction solubilities in binary solvent mixtures-Continued

\begin{tabular}{lrc}
\hline \hline Solvent (2) + solvent (3) & $S_{23, i}{ }^{\mathrm{a}}$ & $\% \mathrm{Dev}^{\mathrm{b}}$ \\
\hline 2,2,4-Trimethylpentane (2) + 1-pentanol (3) & 0.530 & 0.5 \\
0.242 & \\
-0.011 &
\end{tabular}

Alkane + ether binary mixtures

Heptane (2) + 1,1'-oxybisbutane (3)

Cyclohexane (2) $+1,1^{\prime}$-oxybisbutane (3)

$0.220 \quad 0.3$

$0.467 \quad 0.3$

2,2,4-Trimethylpentane (2) +1,1'-oxybisbutane (3)

0.190

0.353

$-0.026$

Heptane (2) + 2-methoxy-2-methylpropane (3)

Cyclohexane (2) + 2-methoxy-2-methylpropane (3)

$-0.268$

0.741

0.174

$-0.305$

2,2,4-Trimethylpentane (2) + 2-methoxy-2-

methylpropane (3)

Heptane (2) + 1,4-dioxane (3)

Cyclohexane (2) + 1,4-dioxane (3)

2,2,4-Trimethylpentane (2) + 1,4-dioxane (3)

Alkane + alkoxyalcohol mixtures

Heptane (2) + 2-ethoxyethanol (3)

Cyclohexane (2) + 2-ethoxyethanol (3)

2,2,4-Trimethylpentane (2) + 2-ethoxyethanol (3)

Heptane (2) + 2-butoxyethanol (3)

Cyclohexane (2) + 2-butoxyethanol (3)

2,2,4-Trimethylpentane (2) + 2-butoxyethanol (3)

Alcohol + alcohol binary mixtures

1-Butanol (2) + 1-propanol (3)

2-Propanol (2) + 1-propanol (3)

2-Butanol (2) + 1-propanol (3)

1-Butanol (2) + 2-propanol (3)

2-Butanol (2) + 2-propanol (3)

2-Butanol (2) + 2-methyl-1-propanol (3)

1-Propanol (2) + 2-methyl-1-propanol (3)

2-Methyl-1-proapnol (2) + 2-propanol (3)

1-Propanol (2) + 1-pentanol (3)
TABLE 1. Combined NIBS/Redlich-Kister $S_{23, i}$ parameters for mathematical representation of anthracene mole fraction solubilities in binary solvent mixtures-Continued

\begin{tabular}{lcc}
\hline \hline Solvent (2) + solvent (3) & $S_{23, i}{ }^{\mathrm{a}}$ & $\% \mathrm{Dev}^{\mathrm{b}}$ \\
\hline 2-Propanol (2) + 1-pentanol (3) & 0.445 & 0.3 \\
& 0.111 & \\
& 0.033 &
\end{tabular}

1-Butanol (2) + 1-pentanol (3)

0.055

0.033

$-0.016$

2-Butanol (2) + 1-pentanol (3)

0.106

$-0.054$

$-0.035$

2-Methyl-1-propanol (2) + 1-pentanol (3)

0.267

0.044

0.078

Alcohol + ether binary mixtures

1-Propanol (2) + 1,1'-oxybisbutane (3)

2.167

0.931

0.891

2.588

1.235

0.866

1.736

0.488

0.574

2.109

0.849

0.726

2.231

0.932

0.929

1.688

0.283

0.272

1.976

0.514

0.651

1.305

0.190

0.282

1.580

0.272

0.294

1.652

2-Methyl-1-propanol (2) + 2-methoxy-2methylpropane (3)

0.2

0.3

0.3

1.1

0.8

0.9

0.6

0.2

0.5

0.2

0.3

0.4

0.428

0.353

2.308

1.305

0.112

2.559

1.745

0.748

1.792

1.140

$-0.330$

2.178

1.504

0.709

2.326

1.232

0.210

Alcohol + alkoxyalcohol binary mixtures

1-Propanol (2) + 2-butoxyethanol (3)

1.222

0.572

0.255
0.8

0.8

0.7

0.9

0.5

0.3 
TABLE 1. Combined NIBS/Redlich-Kister $S_{23, i}$ parameters for mathematical representation of anthracene mole fraction solubilities in binary solvent mixtures-Continued

\begin{tabular}{lcc}
\hline \hline Solvent (2) + solvent (3) & $S_{23, i}{ }^{\mathrm{a}}$ & $\% \mathrm{Dev}^{\mathrm{b}}$ \\
\hline 2-Propanol (2) + 2-butoxyethanol (3) & 1.550 & 0.6 \\
& 0.790 & \\
& 0.726 & \\
1-Butanol (2) + 2-butoxyethanol (3) & 0.800 & 0.5 \\
& 0.319 & \\
& 0.185 & \\
2-Butanol (2) + 2-butoxyethanol (3) & 1.155 & 0.3 \\
& 0.609 & \\
& 0.267 & \\
\hline
\end{tabular}

${ }^{\mathrm{a}}$ Combined NIBS/Redlich-Kister curve-fit parameters are ordered as $S_{23,0}$, $S_{23,1}$, and $S_{23,2}$. If only a single coefficient is listed, then both $S_{23,1}$ and $S_{23,2}$ are zero. Similarly, if two coefficients are listed, then $S_{23,2}$ is zero.

${ }^{\mathrm{b}} \operatorname{Dev}(\%)=(100 / N) \Sigma\left|\left[x_{1}{ }^{\exp }-x_{1}{ }^{\mathrm{cal}}\right] / x_{1}{ }^{\exp }\right|$.

The popularity of the Combined NIBS/Redlich-Kister model results from the fact that the computed $S_{I J, i}$ parameters can be used to predict solute solubility in ternary solvent systems

$$
\begin{aligned}
\ln x_{1}{ }^{\mathrm{sat}}= & x_{2}{ }^{(\mathrm{s})} \ln \left(x_{1}{ }^{\mathrm{sat}}\right)_{2}+x_{3}{ }^{(\mathrm{s})} \ln \left(x_{1}{ }^{\mathrm{sat}}\right)_{3}+x_{4}{ }^{(\mathrm{s})} \ln \left(x_{1}{ }^{\mathrm{sat}}\right)_{4} \\
& +x_{2}{ }^{(\mathrm{s})} x_{3}{ }^{(\mathrm{s})} \sum_{j=0}^{r} S_{23, j}\left(x_{2}{ }^{(\mathrm{s})}-x_{3}{ }^{(\mathrm{s})}\right)^{j} \\
& +x_{2}{ }^{(\mathrm{s})} x_{4}{ }^{(\mathrm{s})} \sum_{k=0}^{s} S_{24, k}\left(x_{2}{ }^{(\mathrm{s})}-x_{4}{ }^{(\mathrm{s})}\right)^{k} \\
& +x_{3}{ }^{(\mathrm{s})} x_{4}{ }^{(\mathrm{s})} \sum_{l=0}^{t} S_{34, l}\left(x_{3}{ }^{(\mathrm{s})}-x_{4}{ }^{(\mathrm{s})}\right)^{l}
\end{aligned}
$$

and in higher order multi-component solvent systems

$$
\ln x_{1}{ }^{\text {sat }}=\sum_{I}^{\text {Solvents }} \sum_{J>I}^{\text {Solvents }}\left[x_{I}^{(\mathrm{s})} x_{J}^{(\mathrm{s})} \sum_{k=0}^{n} S_{I J, i}\left(x_{I}^{(\mathrm{s})}-x_{J}^{(\mathrm{s})}\right)^{k}\right] .
$$

Equation (2) is referred to as the Combined Nearly Ideal Ternary Solvent (NITS)/Redlich-Kister model. To date, Eq. (2) has been shown to provide very accurate predictions for the solubility of anthracene and/or pyrene in 114 different ternary solvent mixtures including several alcohol + hydrocarbon + hydrocarbon, alcohol + alcohol + hydrocarbon, alkoxyalcohol + alcohol + hydrocarbon, alkoxyalcohol + alcohol + alcohol, and alkyl ether + alcohol + hydrocarbon solvent systems. ${ }^{7-34}$ The aforementioned solubility data are included in this part of Vol. 98.

\section{Solubility of Anthracene in Ternary} Alkane + Alkane + Alcohol Solvent Mixtures

\subsection{Critical evaluation of experimental solubility data}

Deng, Acree, and co-workers ${ }^{23,27}$ reported the solubility of

\begin{tabular}{|c|c|c|}
\hline Solvent $(2)+$ solvent $(3)$ & $S_{23, i}{ }^{\mathrm{a}}$ & $\% \operatorname{Dev}^{\mathrm{b}}$ \\
\hline \multicolumn{3}{|l|}{ Alkane + alkane binary mixtures } \\
\hline Cyclohexane (2) + heptane (3) & 0.232 & 0.4 \\
\hline \multicolumn{3}{|l|}{ Alkane + alcohol binary mixtures } \\
\hline \multirow[t]{3}{*}{ Heptane (2) + 1-propanol (3) } & 1.383 & 0.4 \\
\hline & -0.202 & \\
\hline & 0.280 & \\
\hline \multirow[t]{3}{*}{ Heptane (2) + 2-propanol (3) } & 1.799 & 0.2 \\
\hline & -0.478 & \\
\hline & 0.581 & \\
\hline \multirow[t]{3}{*}{ Heptane (2) + 1-butanol (3) } & 1.075 & 0.3 \\
\hline & 0.189 & \\
\hline & 0.388 & \\
\hline \multirow{3}{*}{ Heptane (2) + 2-butanol (3) } & 1.583 & 0.5 \\
\hline & -0.232 & \\
\hline & 0.392 & \\
\hline \multirow[t]{3}{*}{ Heptane (2) + 2-methyl-1-propanol (3) } & 1.604 & 0.4 \\
\hline & -0.151 & \\
\hline & 0.545 & \\
\hline \multirow[t]{3}{*}{ Cyclohexane (2) + 1-propanol (3) } & 1.396 & 0.7 \\
\hline & 0.268 & \\
\hline & 0.226 & \\
\hline \multirow[t]{3}{*}{ Cyclohexane (2) + 2-propanol (3) } & 1.726 & 0.1 \\
\hline & -0.165 & \\
\hline & 0.372 & \\
\hline \multirow[t]{3}{*}{ Cyclohexane (2) + 1-butanol (3) } & 0.976 & 0.6 \\
\hline & 0.462 & \\
\hline & 0.472 & \\
\hline \multirow[t]{3}{*}{ Cyclohexane (2) + 2-butanol (3) } & 1.426 & 0.5 \\
\hline & 0.064 & \\
\hline & 0.447 & \\
\hline \multirow[t]{3}{*}{ Cyclohexane (2) + 2-methyl-1-propanol (3) } & 1.316 & 0.6 \\
\hline & 0.427 & \\
\hline & 0.190 & \\
\hline \multirow[t]{3}{*}{ 2,2,4-Trimethylpentane (2) + 1-propanol (3) } & 0.864 & 0.1 \\
\hline & -0.255 & \\
\hline & 0.304 & \\
\hline \multirow[t]{3}{*}{ 2,2,4-Trimethylpentane (2) + 2-propanol (3) } & 1.329 & 0.5 \\
\hline & -0.232 & \\
\hline & 0.378 & \\
\hline \multirow[t]{3}{*}{ 2,2,4-Trimethylpentane (2) + 1-butanol (3) } & 0.826 & 0.3 \\
\hline & 0.105 & \\
\hline & 0.875 & \\
\hline \multirow[t]{3}{*}{ 2,2,4-Trimethylpentane (2) + 2-butanol (3) } & 1.148 & 0.7 \\
\hline & -0.274 & \\
\hline & 0.558 & \\
\hline \multicolumn{3}{|l|}{ Alcohol + alcohol binary mixtures } \\
\hline \multirow[t]{2}{*}{ 1-Butanol (2) + 1-propanol (3) } & 0.127 & 0.4 \\
\hline & 0.065 & \\
\hline 2-Butanol (2) + 1-propanol (3) & 0.000 & 0.5 \\
\hline 1-Butanol (2) + 2-propanol (3) & 0.215 & 0.5 \\
\hline 2-Butanol (2) + 2-propanol (3) & 0.270 & 0.3 \\
\hline
\end{tabular}
anthracene in ternary heptane + cyclohexane + alcohol and
TABLE 2. Combined NIBS/Redlich-Kister $S_{23, i}$ parameters for mathematical representation of pyrene mole fraction solubilities in binary solvent mixtures

${ }^{a}$ Combined NIBS/Redlich-Kister curve-fit parameters are ordered as $S_{23,0}$, $S_{23,1}$, and $S_{23,2}$. If only a single coefficient is listed, then both $S_{23,1}$ and $S_{23,2}$ are zero. Similarly, if two coefficients are listed, then $S_{23,2}$ is zero.

${ }^{\mathrm{b}} \operatorname{Dev}(\%)=(100 / N) \Sigma\left|\left[x_{1}{ }^{\mathrm{exp}}-x_{1}{ }^{\mathrm{cal}}\right] / x_{1}{ }^{\mathrm{exp}}\right|$.

2,2,4-trimethylpentane + cyclohexane + alcohol solvent mixtures at $298.15 \mathrm{~K}$ based on spectrophotometric measurements. The specific alcohol solvents studied were 1-propanol, 2propanol, 1-butanol, and 2-butanol. There has only been a single experimental determination for the solubility of anthracene in each of the ternary solvent systems studied. The 
TABLE 3. Summarized comparison between experimental solubilities of anthracene in ternary alkane + alkane + alcohol solvent mixtures and predicted values based on Eq. (2)

\begin{tabular}{lc}
\hline \hline Ternary solvent mixture & \% Dev. \\
\hline Heptane + cyclohexane + 1-propanol & 1.55 \\
Heptane + cyclohexane + 2-propanol & 0.92 \\
Heptane + cyclohexane + 1-butanol & 1.11 \\
Heptane + cyclohexane + 2-butanol & 1.58 \\
Cyclohexane + 2,2,4-trimethylpentane + 1-propanol & 1.60 \\
Cyclohexane + 2,2,4-trimethylpentane + 2-propanol & 1.40 \\
Cyclohexane $+2,2,4$-trimethylpentane +1 -butanol & 1.70 \\
Cyclohexane $+2,2,4-$ trimethylpentane +2 -butanol & 1.71 \\
\hline a Dev $(\%)$
\end{tabular}

experimental values were evaluated by curve-fitting the measured solubility data for the respective binary sub-systems to the Combined NIBS/Redlich-Kister equation, and then using the ternary solvent version of the basic model, Eq. (2), to estimate the mole fraction solubility of anthracene dissolved in ternary alkane + alkane + alcohol solvent mixtures.

The calculated $S_{23, i}$ parameters for all of the contributing binary alkane + alkane and alkane + alcohol solvent systems studied are summarized in Table 1, along with the average absolute deviation between the experimental and back-calculated mole fraction solubilities. The numerical values of the curve-fit parameters were determined through analysis of measured anthracene solubilities in the respective binary solvent system. Experimental solubility data for the binary solvents are contained in Vols. 54 and 58 of the IUPAC Solubility Data Series, ${ }^{1,2}$ as well as in Part 1 of this volume. ${ }^{4}$ The predictive ability of Eq. (2) is given in Table 3 for the eight ternary alkane + alkane + alcohol solvent systems. Examination of the numerical entries in the last column of Table 3 reveals that Eq. (2) does provide a fairly accurate prediction for how the measured mole fraction solubilities vary with ternary solvent composition. Average percent deviations range from $0.9 \%$ to $1.7 \%$, suggesting that there are no obvious outliers in an individual ternary solvent data set. As an informational note, the experimental uncertainties associated with the measured mole fraction solubilities were \pm 1.5 relative percent. None of the experimental data points was flagged as an outlier.

The experimental anthracene solubility data for ternary heptane + cyclohexane + alcohol and 2,2,4-trimethylpentane + cyclohexane + alcohol solvent mixtures are given in Secs. 2.2 and 2.3 .

\subsection{Anthracene solubility data in ternary heptane + cyclohexane + alcohol solvent mixtures}

\begin{tabular}{ll}
\hline \hline Components: & Original Measurements: \\
(1) Anthracene; $\mathrm{C}_{14} \mathrm{H}_{10} ;[120-12-7]$ & ${ }^{23}$ T. Deng, C. E. Hernández, L. E. \\
(2) Heptane; $\mathrm{C}_{7} \mathrm{H}_{16} ;[142-82-5]$ & Roy, and W. E. Acree, Jr., J. \\
(3) Cyclohexane; $\mathrm{C}_{6} \mathrm{H}_{12} ;[110-82-7]$ & Chem. Thermodyn. 31, 205 \\
(4) 1-Propanol; $\mathrm{C}_{3} \mathrm{H}_{8} \mathrm{O} ;[71-23-8]$ & $(1999)$. \\
\hline Variables: & Prepared by: \\
$T / \mathrm{K}=298.15 ;$ Solvent Composition & W. E. Acree, Jr. \\
\hline
\end{tabular}

Experimental Values

Solubility of anthracene in heptane + cyclohexane +1 -propanol mixtures ${ }^{\mathrm{a}}$

\begin{tabular}{lcccc}
\hline \hline$x_{2}{ }^{(\mathrm{s})}$ & $x_{4}{ }^{(\mathrm{s})}$ & $x_{2}$ & $x_{4}$ & $x_{1}$ \\
\hline 0.0000 & 0.0000 & 0.0000 & 0.0000 & 0.001553 \\
1.0000 & 0.0000 & 0.9984 & 0.0000 & 0.001571 \\
0.0000 & 1.0000 & 0.0000 & 0.9994 & 0.000591 \\
0.2324 & 0.4559 & 0.2321 & 0.4553 & 0.001324 \\
0.5809 & 0.2488 & 0.5800 & 0.2484 & 0.001508 \\
0.1782 & 0.3468 & 0.1779 & 0.3463 & 0.001448 \\
0.3708 & 0.3746 & 0.3703 & 0.3471 & 0.001397 \\
0.0573 & 0.7904 & 0.0573 & 0.7897 & 0.000863 \\
0.0731 & 0.7955 & 0.0730 & 0.7948 & 0.000849 \\
0.5713 & 0.3181 & 0.5705 & 0.3176 & 0.001451 \\
0.4012 & 0.1618 & 0.4006 & 0.1615 & 0.001591 \\
0.3482 & 0.5577 & 0.3478 & 0.5570 & 0.001202 \\
0.2589 & 0.6509 & 0.2586 & 0.6502 & 0.001071 \\
0.1117 & 0.2138 & 0.1115 & 0.2135 & 0.001550 \\
0.0866 & 0.7944 & 0.0865 & 0.7937 & 0.000871 \\
0.1618 & 0.6254 & 0.1616 & 0.6247 & 0.001115 \\
0.1512 & 0.1564 & 0.1510 & 0.1562 & 0.001574 \\
0.5981 & 0.1721 & 0.5972 & 0.1718 & 0.001532 \\
0.0692 & 0.2779 & 0.0691 & 0.2775 & 0.001506 \\
0.3127 & 0.1581 & 0.3122 & 0.1578 & 0.001603 \\
0.0646 & 0.5022 & 0.0645 & 0.5016 & 0.001276 \\
0.0642 & 0.6044 & 0.0641 & 0.6037 & 0.001125 \\
\hline
\end{tabular}

${ }^{\mathrm{a}} x_{2}{ }^{(\mathrm{s})}$ : initial mole fraction of component 2 in the ternary solvent mixture, $x_{4}{ }^{(\mathrm{s})}$ : initial mole fraction of component 4 in the ternary solvent mixture; $x_{1}$ : mole fraction solubility of the solute; $x_{2}$ : mole fraction of component 2 in the quaternary solution; $x_{4}$ : mole fraction of component 4 in the quaternary solution.

\section{Auxiliary Information}

\section{Method/Apparatus/Procedure:}

Constant-temperature bath, calorimetric thermometer, and an ultraviolet/ visible spectrophotometer.

Ternary solvent mixtures were prepared by mass. Excess solute and solvent were placed in amber glass bottles and allowed to equilibrate for several days at constant temperature. Attainment of equilibrium was verified by several repetitive measurements and by approaching equilibrium from supersaturation. Aliquots of saturated solutions were transferred through a coarse filter into volumetric flasks, weighed and diluted with methanol. Molar concentrations were determined by spectrophotometric measurements at $356 \mathrm{~nm}$.

\section{Source and Purity of Chemicals:}

(1) $99+\%$, Acros Chemical Company, USA, recrystallized three times from acetone.

(2) $99 \%$, HPLC grade, Aldrich Chemical Company, Milwaukee, WI, USA

(3) $99.9 \%$, HPLC grade, Aldrich Chemical Company.

(4) $99+\%$, anhydrous, Aldrich Chemical Company.

Components 2-4 were stored over molecular sieves and distilled shortly before use.

\section{Estimated Error:}

Temperature: $\pm 0.1 \mathrm{~K}$.

$x_{2}{ }^{(\mathrm{s})}: \pm 0.0001$.

$x_{4}^{(\mathrm{s})}: \pm 0.0001$.

$x_{1}: \pm 1.5 \%$ (relative error) 


\section{Components:}

(1) Anthracene; $\mathrm{C}_{14} \mathrm{H}_{10} ;[120-12-7]$

(2) Heptane; $\mathrm{C}_{7} \mathrm{H}_{16} ;[142-82-5]$

(3) Cyclohexane; $\mathrm{C}_{6} \mathrm{H}_{12} ;[110-82-7]$

(4) 2-Propanol; $\mathrm{C}_{3} \mathrm{H}_{8} \mathrm{O}$; [67-63-0]

\begin{tabular}{ll}
\hline Variables: & Prepared by: \\
$T / \mathrm{K}=298.15 ;$ Solvent Composition & W. E. Acree, Jr. \\
\hline
\end{tabular}

\section{Experimental Values}

Solubility of anthracene in heptane + cyclohexane +2 -propanol mixtures ${ }^{\mathrm{a}}$

\begin{tabular}{lcccc}
\hline \hline$x_{2}{ }^{(\mathrm{s})}$ & $x_{4}{ }^{(\mathrm{s})}$ & $x_{2}$ & $x_{4}$ & $x_{1}$ \\
\hline 0.0000 & 0.0000 & 0.0000 & 0.0000 & 0.001553 \\
1.0000 & 0.0000 & 0.9984 & 0.0000 & 0.001571 \\
0.0000 & 1.0000 & 0.0000 & 0.9996 & 0.000411 \\
0.2309 & 0.4479 & 0.2306 & 0.4473 & 0.001282 \\
0.5780 & 0.2467 & 0.5771 & 0.2463 & 0.001499 \\
0.1734 & 0.3396 & 0.1732 & 0.3391 & 0.001421 \\
0.3738 & 0.3640 & 0.3733 & 0.3635 & 0.001355 \\
0.0601 & 0.7813 & 0.0601 & 0.7807 & 0.000760 \\
0.1196 & 0.7989 & 0.1195 & 0.7983 & 0.000743 \\
0.5751 & 0.3117 & 0.5743 & 0.3113 & 0.001418 \\
0.4050 & 0.1600 & 0.4044 & 0.1597 & 0.001565 \\
0.3607 & 0.5443 & 0.3603 & 0.5437 & 0.001129 \\
0.2655 & 0.6429 & 0.2652 & 0.6423 & 0.000991 \\
0.1082 & 0.2133 & 0.1080 & 0.2130 & 0.001543 \\
0.0882 & 0.7895 & 0.0881 & 0.7889 & 0.000750 \\
0.1627 & 0.6171 & 0.1610 & 0.6165 & 0.001043 \\
0.1486 & 0.1459 & 0.1484 & 0.1457 & 0.001595 \\
0.6020 & 0.1681 & 0.6011 & 0.1678 & 0.001543 \\
0.0724 & 0.2730 & 0.0723 & 0.2726 & 0.001488 \\
0.3150 & 0.1534 & 0.3145 & 0.1532 & 0.001588 \\
0.0675 & 0.4939 & 0.0674 & 0.4933 & 0.001199 \\
0.0656 & 0.6000 & 0.0655 & 0.5994 & 0.001031 \\
\hline
\end{tabular}

${ }^{\mathrm{a}} x_{2}{ }^{(\mathrm{s})}$ : initial mole fraction of component 2 in the ternary solvent mixture, $x_{4}{ }^{(\mathrm{s})}$ initial mole fraction of component 4 in the ternary solvent mixture; $x_{1}$ : mole fraction solubility of the solute; $x_{2}$ : mole fraction of component 2 in the quaternary solution; $x_{4}$ : mole fraction of component 4 in the quaternary solution.

\section{Auxiliary Information}

\section{Method/Apparatus/Procedure:}

Constant-temperature bath, calorimetric thermometer, and an ultraviolet/ visible spectrophotometer.

Ternary solvent mixtures were prepared by mass. Excess solute and solvent were placed in amber glass bottles and allowed to equilibrate for several days at constant temperature. Attainment of equilibrium was verified by several repetitive measurements and by approaching equilibrium from supersaturation. Aliquots of saturated solutions were transferred through a coarse filter into volumetric flasks, weighed and diluted with methanol. Molar concentrations were determined by spectrophotometric measurements at $356 \mathrm{~nm}$.

\section{Source and Purity of Chemicals:}

(1) $99+\%$, Acros Chemical Company, USA, recrystallized three times from acetone.

(2) $99 \%$, HPLC grade, Aldrich Chemical Company, Milwaukee, WI, USA.

(3) $99.9 \%$, HPLC grade, Aldrich Chemical Company.

(4) $99+\%$, anhydrous, Aldrich Chemical Company.

Components $2-4$ were stored over molecular sieves and distilled shortly before use.
Estimated Error:

Temperature: $\pm 0.1 \mathrm{~K}$.

$x_{2}{ }^{(\mathrm{s})}: \pm 0.0001$.

$x_{4}{ }^{(\mathrm{s})}: \pm 0.0001$.

$x_{1}: \pm 1.5 \%$ (relative error).

\begin{tabular}{ll}
\hline \hline Components: & $\begin{array}{l}\text { Original Measurements: } \\
\text { (1) Anthracene; } \mathrm{C}_{14} \mathrm{H}_{10} ;[120-12-7]\end{array}$ \\
${ }^{23}$ T. Deng, C. E. Hernández, L. E. \\
(2) Heptane; $\mathrm{C}_{7} \mathrm{H}_{16} ;[142-82-5]$ & Roy, and W. E. Acree, Jr., J. \\
(3) Cyclohexane; $\mathrm{C}_{6} \mathrm{H}_{12} ;[110-82-7]$ & Chem. Thermodyn. 31, 205 \\
(4) 1-Butanol; $\mathrm{C}_{4} \mathrm{H}_{10} \mathrm{O} ;[71-36-3]$ & $(1999)$. \\
\hline Variables: & Prepared by: \\
$T / \mathrm{K}=298.15 ;$ Solvent Composition & W. E. Acree, Jr. \\
\hline
\end{tabular}

Experimental Values

Solubility of anthracene in heptane + cyclohexane +1 -butanol mixtures ${ }^{\mathrm{a}}$

\begin{tabular}{|c|c|c|c|c|}
\hline$x_{2}^{(\mathrm{s})}$ & $x_{4}{ }^{(s)}$ & $x_{2}$ & $x_{4}$ & $x_{1}$ \\
\hline 0.0000 & 0.0000 & 0.0000 & 0.0000 & 0.001553 \\
\hline 1.0000 & 0.0000 & 0.9984 & 0.0000 & 0.001571 \\
\hline 0.0000 & 1.0000 & 0.0000 & 0.9992 & 0.000801 \\
\hline 0.2496 & 0.4089 & 0.2492 & 0.4083 & 0.001492 \\
\hline 0.6053 & 0.2147 & 0.6043 & 0.2144 & 0.001589 \\
\hline 0.1822 & 0.2968 & 0.1819 & 0.2963 & 0.001575 \\
\hline 0.4014 & 0.3226 & 0.4008 & 0.3221 & 0.001540 \\
\hline 0.0707 & 0.7475 & 0.0706 & 0.7467 & 0.001086 \\
\hline 0.1370 & 0.7687 & 0.1369 & 0.7679 & 0.001071 \\
\hline 0.6005 & 0.2798 & 0.5996 & 0.2794 & 0.001542 \\
\hline 0.4223 & 0.1291 & 0.4216 & 0.1289 & 0.001606 \\
\hline 0.3954 & 0.4974 & 0.3949 & 0.4967 & 0.001358 \\
\hline 0.2993 & 0.5949 & 0.2989 & 0.5941 & 0.001266 \\
\hline 0.1127 & 0.1842 & 0.1125 & 0.1839 & 0.001625 \\
\hline 0.1002 & 0.7618 & 0.1001 & 0.7610 & 0.001075 \\
\hline 0.1970 & 0.6402 & 0.1968 & 0.6394 & 0.001220 \\
\hline 0.1534 & 0.1232 & 0.1531 & 0.1230 & 0.001643 \\
\hline 0.6183 & 0.1437 & 0.6173 & 0.1435 & 0.001590 \\
\hline 0.0730 & 0.2331 & 0.0729 & 0.2327 & 0.001573 \\
\hline 0.3221 & 0.1308 & 0.3216 & 0.1306 & 0.001623 \\
\hline 0.0721 & 0.4499 & 0.0720 & 0.4493 & 0.001394 \\
\hline 0.0713 & 0.5535 & 0.0712 & 0.5528 & 0.001293 \\
\hline
\end{tabular}

solution.

\section{Auxiliary Information}

\section{Method/Apparatus/Procedure:}

Constant-temperature bath, calorimetric thermometer, and an ultraviolet/ visible spectrophotometer.

Ternary solvent mixtures were prepared by mass. Excess solute and solvent were placed in amber glass bottles and allowed to equilibrate for several days at constant temperature. Attainment of equilibrium was verified by several repetitive measurements and by approaching equilibrium from supersaturation. Aliquots of saturated solutions were transferred through a coarse filter into volumetric flasks, weighed and diluted with methanol. Molar concentrations were determined by spectrophotometric measurements at $356 \mathrm{~nm}$. 
Source and Purity of Chemicals:

(1) $99+\%$, Acros Chemical Company, USA, recrystallized three times from acetone.

(2) $99 \%$, HPLC grade, Aldrich Chemical Company, Milwaukee, WI, USA.

(3) $99.9 \%$, HPLC grade, Aldrich Chemical Company.

(4) $99.8 \%$, HPLC grade, Aldrich Chemical Company.

Components 2-4 were stored over molecular sieves and distilled shortly before use.

Estimated Error:

Temperature: $\pm 0.1 \mathrm{~K}$.

$x_{2}{ }^{(\mathrm{s})}: \pm 0.0001$.

$x_{4}{ }^{(\mathrm{s})}: \pm 0.0001$.

$x_{1}: \pm 1.5 \%$ (relative error).

\begin{tabular}{ll}
\hline \hline Components: & Original Measurements: \\
(1) Anthracene; $\mathrm{C}_{14} \mathrm{H}_{10} ;[120-12-7]$ & ${ }^{23}$ T. Deng, C. E. Hernández, L. E. \\
(2) Heptane; $\mathrm{C}_{7} \mathrm{H}_{16} ;[142-82-5]$ & Roy, and W. E. Acree, Jr., J. \\
(3) Cyclohexane; $\mathrm{C}_{6} \mathrm{H}_{12} ;[110-82-7]$ & Chem. Thermodyn. 31, 205 \\
(4) 2-Butanol; $\mathrm{C}_{4} \mathrm{H}_{10} \mathrm{O} ;[78-92-2]$ & $(1999)$. \\
\hline Variables: & Prepared by: \\
$T / \mathrm{K}=298.15 ;$ Solvent Composition & W. E. Acree, Jr. \\
\hline
\end{tabular}

Experimental Values

Solubility of anthracene in heptane + cyclohexane +2 -butanol mixtures ${ }^{a}$

\begin{tabular}{lcccc}
\hline \hline$x_{2}{ }^{(\mathrm{s})}$ & $x_{4}{ }^{(\mathrm{s})}$ & $x_{2}$ & $x_{4}$ & $x_{1}$ \\
\hline 0.0000 & 0.0000 & 0.0000 & 0.0000 & 0.001553 \\
1.0000 & 0.0000 & 0.9984 & 0.0000 & 0.001571 \\
0.0000 & 1.0000 & 0.0000 & 0.9994 & 0.000585 \\
0.2629 & 0.3962 & 0.2625 & 0.3956 & 0.001423 \\
0.5758 & 0.1899 & 0.5749 & 0.1896 & 0.001541 \\
0.1977 & 0.2970 & 0.1974 & 0.2965 & 0.001530 \\
0.4269 & 0.3064 & 0.4263 & 0.3059 & 0.001491 \\
0.0567 & 0.7659 & 0.0566 & 0.7652 & 0.000933 \\
0.1262 & 0.7855 & 0.1261 & 0.7848 & 0.000914 \\
0.6115 & 0.2804 & 0.6106 & 0.2800 & 0.001525 \\
0.4138 & 0.1328 & 0.4131 & 0.1326 & 0.001613 \\
0.3891 & 0.5063 & 0.3886 & 0.5056 & 0.001297 \\
0.2923 & 0.6053 & 0.2920 & 0.6046 & 0.001163 \\
0.1225 & 0.1777 & 0.1223 & 0.1774 & 0.001583 \\
0.1175 & 0.8183 & 0.1174 & 0.8176 & 0.000883 \\
0.1896 & 0.5648 & 0.1894 & 0.5641 & 0.001217 \\
0.1635 & 0.1195 & 0.1632 & 0.1193 & 0.001604 \\
0.6388 & 0.1333 & 0.6378 & 0.1331 & 0.001570 \\
0.0732 & 0.2261 & 0.0731 & 0.2257 & 0.001577 \\
0.3187 & 0.1234 & 0.3162 & 0.1232 & 0.001640 \\
0.0655 & 0.4488 & 0.0654 & 0.4482 & 0.001369 \\
0.0662 & 0.5604 & 0.0661 & 0.5597 & 0.001197 \\
\hline${ }^{a} x_{2}{ }^{(\mathrm{s})}:$ initial mole fraction of component 2 in the ternary solvent mixture, $x_{4}{ }^{(\mathrm{s})}$ : \\
initial mole fraction of component 4 in the ternary solvent mixture; $x_{1}:$ mole \\
fraction solubility of the solute; $x_{2}:$ mole fraction of component 2 in the \\
quaternary solution $; x_{4}:$ mole fraction of component 4 in the quaternary \\
solution. & & & & \\
& & & &
\end{tabular}

\section{Auxiliary Information}

Method/Apparatus/Procedure:

Constant-temperature bath, calorimetric thermometer, and an ultraviolet/ visible spectrophotometer.

Ternary solvent mixtures were prepared by mass. Excess solute and solvent were placed in amber glass bottles and allowed to equilibrate for several days at constant temperature. Attainment of equilibrium was verified by several repetitive measurements and by approaching equilibrium from supersaturation. Aliquots of saturated solutions were transferred through a coarse filter into volumetric flasks, weighed and diluted with methanol. Molar concentrations were determined by spectrophotometric measurements at $356 \mathrm{~nm}$.

Source and Purity of Chemicals:

(1) $99+\%$, Acros Chemical Company, USA, recrystallized three times from acetone.

(2) $99 \%$, HPLC grade, Aldrich Chemical Company, Milwaukee, WI, USA.

(3) $99.9 \%$, HPLC grade, Aldrich Chemical Company.

(4) $99+\%$, anhydrous, Aldrich Chemical Company.

Components $2-4$ were stored over molecular sieves and distilled shortly before use.

Estimated Error:

Temperature: $\pm 0.1 \mathrm{~K}$.

$x_{2}{ }^{(\mathrm{s})}: \pm 0.0001$.

$x_{4}{ }^{(\mathrm{s})}: \pm 0.0001$.

$x_{1}: \pm 1.5 \%$ (relative error)

\subsection{Anthracene solubility data in ternary $2,2,4-$ trimethylpentane + cyclohexane + alcohol solvent mixtures}

\begin{tabular}{|c|c|}
\hline $\begin{array}{l}\text { Components: } \\
\text { (1) Anthracene; } \mathrm{C}_{14} \mathrm{H}_{10} ;[120-12-7] \\
\text { (2) } 2,2,4-\text { Trimethylpentane; } \mathrm{C}_{8} \mathrm{H}_{18} \text {; } \\
\text { [540-84-1] } \\
\text { (3) Cyclohexane; } \mathrm{C}_{6} \mathrm{H}_{12} ;[110-82-7] \\
\text { (4) 1-Propanol; } \mathrm{C}_{3} \mathrm{H}_{8} \mathrm{O} ;[71-23-8]\end{array}$ & $\begin{array}{l}\text { Original Measurements: } \\
{ }^{27} \text { T. Deng and W. E. Acree, Jr., J. } \\
\text { Chem. Eng. Data 43, } 1059 \text { (1998). }\end{array}$ \\
\hline $\begin{array}{l}\text { Variables: } \\
T / \mathrm{K}=298.15 \text {; Solvent Composition }\end{array}$ & $\begin{array}{l}\text { Prepared by: } \\
\text { W. E. Acree, Jr. }\end{array}$ \\
\hline
\end{tabular}


Experimental Values

Solubility of anthracene in 2,2,4-trimethylpentane + cyclohexane +1 -propanol mixtures $^{\mathrm{a}}$

\begin{tabular}{lcccc}
\hline \hline$x_{2}{ }^{(\mathrm{s})}$ & $x_{4}{ }^{(\mathrm{s})}$ & $x_{2}$ & $x_{4}$ & $x_{1}$ \\
\hline 0.0000 & 0.0000 & 0.0000 & 0.0000 & 0.001553 \\
1.0000 & 0.0000 & 0.9989 & 0.0000 & 0.001074 \\
0.0000 & 1.0000 & 0.0000 & 0.9994 & 0.000591 \\
0.2145 & 0.4603 & 0.2142 & 0.4598 & 0.001175 \\
0.5536 & 0.2597 & 0.5330 & 0.2594 & 0.001180 \\
0.1606 & 0.3512 & 0.1604 & 0.3507 & 0.001346 \\
0.3479 & 0.3826 & 0.3475 & 0.3821 & 0.001190 \\
0.0500 & 0.7937 & 0.0500 & 0.7930 & 0.000875 \\
0.1052 & 0.8129 & 0.1051 & 0.8122 & 0.000815 \\
0.5356 & 0.3443 & 0.5350 & 0.3439 & 0.001133 \\
0.3739 & 0.1695 & 0.3734 & 0.1693 & 0.001351 \\
0.3232 & 0.5746 & 0.3229 & 0.5740 & 0.001027 \\
0.2401 & 0.6654 & 0.2399 & 0.6648 & 0.000952 \\
0.0975 & 0.2112 & 0.0974 & 0.2109 & 0.001515 \\
0.0792 & 0.7983 & 0.0791 & 0.7976 & 0.000831 \\
0.1443 & 0.6334 & 0.1442 & 0.6327 & 0.001033 \\
0.1336 & 0.1517 & 0.1334 & 0.1515 & 0.001515 \\
0.5644 & 0.1860 & 0.5637 & 0.1858 & 0.001235 \\
0.0615 & 0.2748 & 0.0614 & 0.2744 & 0.001504 \\
0.2855 & 0.1643 & 0.2851 & 0.1641 & 0.001405 \\
0.0570 & 0.5069 & 0.0569 & 0.5063 & 0.001236 \\
0.0551 & 0.6086 & 0.0550 & 0.6079 & 0.001119 \\
\hline
\end{tabular}

${ }^{\mathrm{a}} x_{2}{ }^{(\mathrm{s})}$ : initial mole fraction of component 2 in the ternary solvent mixture, $x_{4}{ }^{(\mathrm{s})}$ : initial mole fraction of component 4 in the ternary solvent mixture; $x_{1}$ : mole fraction solubility of the solute; $x_{2}$ : mole fraction of component 2 in the quaternary solution; $x_{4}$ : mole fraction of component 4 in the quaternary solution.

\section{Auxiliary Information}

\section{Method/Apparatus/Procedure:}

Constant-temperature bath, calorimetric thermometer, and an ultraviolet/ visible spectrophotometer.

Ternary solvent mixtures were prepared by mass. Excess solute and solvent were placed in amber glass bottles and allowed to equilibrate for several days at constant temperature. Attainment of equilibrium was verified by several repetitive measurements and by approaching equilibrium from supersaturation. Aliquots of saturated solutions were transferred through a coarse filter into volumetric flasks, weighed and diluted with methanol. Molar concentrations were determined by spectrophotometric measurements at $356 \mathrm{~nm}$.

\section{Source and Purity of Chemicals:}

(1) $99+\%$, Acros Chemical Company, USA, recrystallized three times from acetone.

(2) $99.7 \%$, HPLC grade, Aldrich Chemical Company, Milwaukee, WI, USA. (3) $99.9 \%$, HPLC grade, Aldrich Chemical Company.

(4) $99+\%$, anhydrous, Aldrich Chemical Company.

Components 2-4 were stored over molecular sieves and distilled shortly before use.

\section{Estimated Error:}

Temperature: $\pm 0.1 \mathrm{~K}$.

$x_{2}^{(\mathrm{s})}: \pm 0.0001$.

$x_{4}{ }^{(\mathrm{s})}: \pm 0.0001$.

$x_{1}: \pm 1.5 \%$ (relative error)

\section{Components:}

(1) Anthracene; $\mathrm{C}_{14} \mathrm{H}_{10} ;[120-12-7]$

(2) 2,2,4-Trimethylpentane; $\mathrm{C}_{8} \mathrm{H}_{18}$; [540-84-1]

(3) Cyclohexane; $\mathrm{C}_{6} \mathrm{H}_{12} ;[110-82-7]$

(4) 2-Propanol; $\mathrm{C}_{3} \mathrm{H}_{8} \mathrm{O}$; [67-63-0]

\begin{tabular}{ll}
\hline Variables: & Prepared by: \\
$T / \mathrm{K}=298.15 ;$ Solvent Composition & W. E. Acree, Jr. \\
\hline
\end{tabular}

Experimental Values

Solubility of anthracene in heptane + cyclohexane +2 -propanol mixtures ${ }^{\mathrm{a}}$

\begin{tabular}{lcccc}
\hline \hline$x_{2}{ }^{(\mathrm{s})}$ & $x_{4}{ }^{(\mathrm{s})}$ & $x_{2}$ & $x_{4}$ & $x_{1}$ \\
\hline 0.0000 & 0.0000 & 0.0000 & 0.0000 & 0.001553 \\
1.0000 & 0.0000 & 0.9989 & 0.0000 & 0.001074 \\
0.0000 & 1.0000 & 0.0000 & 0.9996 & 0.000411 \\
0.2148 & 0.4621 & 0.2146 & 0.4616 & 0.001108 \\
0.5549 & 0.2607 & 0.5543 & 0.2604 & 0.001117 \\
0.1612 & 0.3516 & 0.1610 & 0.3512 & 0.001267 \\
0.3501 & 0.3847 & 0.3497 & 0.3843 & 0.001114 \\
0.0533 & 0.7899 & 0.0533 & 0.7894 & 0.000695 \\
0.1065 & 0.8115 & 0.1064 & 0.8110 & 0.000637 \\
0.5378 & 0.3441 & 0.5372 & 0.3437 & 0.001069 \\
0.3764 & 0.1696 & 0.3759 & 0.1694 & 0.001310 \\
0.3340 & 0.5718 & 0.3337 & 0.5713 & 0.000906 \\
0.2447 & 0.6621 & 0.2445 & 0.6616 & 0.000815 \\
0.0982 & 0.2199 & 0.0981 & 0.2196 & 0.001433 \\
0.0799 & 0.8023 & 0.0798 & 0.8018 & 0.000675 \\
0.1468 & 0.6340 & 0.1467 & 0.6334 & 0.000899 \\
0.1350 & 0.1468 & 0.1348 & 0.1466 & 0.001446 \\
0.5706 & 0.1808 & 0.5699 & 0.1806 & 0.001189 \\
0.0633 & 0.2747 & 0.0632 & 0.2743 & 0.001428 \\
0.2927 & 0.1557 & 0.2923 & 0.1555 & 0.001364 \\
0.0585 & 0.4998 & 0.0584 & 0.4992 & 0.001147 \\
0.0566 & 0.6017 & 0.0565 & 0.6011 & 0.000983 \\
\hline
\end{tabular}

${ }^{\mathrm{a}} x_{2}{ }^{(\mathrm{s})}$ : initial mole fraction of component 2 in the ternary solvent mixture, $x_{4}{ }^{(\mathrm{s})}$ : initial mole fraction of component 4 in the ternary solvent mixture; $x_{1}$ : mole fraction solubility of the solute; $x_{2}$ : mole fraction of component 2 in the quaternary solution; $x_{4}$ : mole fraction of component 4 in the quaternary solution.

\section{Auxiliary Information}

\section{Method/Apparatus/Procedure:}

Constant-temperature bath, calorimetric thermometer, and an ultraviolet/ visible spectrophotometer.

Ternary solvent mixtures were prepared by mass. Excess solute and solvent were placed in amber glass bottles and allowed to equilibrate for several days at constant temperature. Attainment of equilibrium was verified by several repetitive measurements and by approaching equilibrium from supersaturation. Aliquots of saturated solutions were transferred through a coarse filter into volumetric flasks, weighed and diluted with methanol. Molar concentrations were determined by spectrophotometric measurements at $356 \mathrm{~nm}$.

Source and Purity of Chemicals:

(1) $99+\%$, Acros Chemical Company, USA, recrystallized three times from acetone.

(2) $99.7 \%$, HPLC grade, Aldrich Chemical Company, Milwaukee, WI, USA. (3) $99.9 \%$, HPLC grade, Aldrich Chemical Company.

(4) $99+\%$, anhydrous, Aldrich Chemical Company.

Components $2-4$ were stored over molecular sieves and distilled shortly before use. 
Estimated Error:

Temperature: $\pm 0.1 \mathrm{~K}$.

$x_{2}^{(\mathrm{s})}: \pm 0.0001$.

$x_{4}{ }^{(\mathrm{s})}: \pm 0.0001$.

$x_{1}: \pm 1.5 \%$ (relative error)

\begin{tabular}{ll}
\hline \hline Components: & $\begin{array}{l}\text { Original Measurements: } \\
\text { (1) Anthracene; } \mathrm{C}_{14} \mathrm{H}_{10} ;[120-12-7]\end{array}$ \\
$\begin{array}{ll}{ }^{27} \mathrm{~T} \text {. Deng and W. E. Acree, Jr., J. } \\
\text { (2) 2,2,4-Trimethylpentane; } \mathrm{C}_{8} \mathrm{H}_{18} ;\end{array}$ & Chem. Eng. Data 43, 1059 (1998). \\
[540-84-1] & \\
(3) Cyclohexane; $\mathrm{C}_{6} \mathrm{H}_{12} ;[110-82-7]$ & \\
(4) 1-Butanol; $\mathrm{C}_{4} \mathrm{H}_{10} \mathrm{O} ;[71-36-3]$ & \\
\hline Variables: & Prepared by: \\
$T / \mathrm{K}=298.15 ;$ Solvent Composition & W. E. Acree, Jr. \\
\hline
\end{tabular}

\section{Experimental Values}

Solubility of anthracene in 2,2,4-trimethylpentane + cyclohexane +1 -butanol mixtures $^{\mathrm{a}}$

\begin{tabular}{lcccc}
\hline \hline$x_{2}{ }^{(\mathrm{s})}$ & $x_{4}{ }^{(\mathrm{s})}$ & $x_{2}$ & $x_{4}$ & $x_{1}$ \\
\hline 0.0000 & 0.0000 & 0.0000 & 0.0000 & 0.001553 \\
1.0000 & 0.0000 & 0.9989 & 0.0000 & 0.001074 \\
0.0000 & 1.0000 & 0.0000 & 0.9992 & 0.000801 \\
0.2251 & 0.4339 & 0.2248 & 0.4333 & 0.001287 \\
0.5821 & 0.2242 & 0.5814 & 0.2239 & 0.001224 \\
0.1717 & 0.3060 & 0.1715 & 0.3056 & 0.001414 \\
0.3762 & 0.3335 & 0.3757 & 0.3331 & 0.001269 \\
0.0612 & 0.7539 & 0.0611 & 0.7531 & 0.001045 \\
0.1246 & 0.7812 & 0.1245 & 0.7804 & 0.000988 \\
0.5749 & 0.2982 & 0.5742 & 0.2978 & 0.001192 \\
0.3869 & 0.1429 & 0.3864 & 0.1428 & 0.001370 \\
0.3620 & 0.5252 & 0.3616 & 0.5246 & 0.001135 \\
0.2737 & 0.6185 & 0.2734 & 0.6178 & 0.001098 \\
0.1027 & 0.1823 & 0.1025 & 0.1820 & 0.001520 \\
0.0928 & 0.7655 & 0.0927 & 0.7647 & 0.001018 \\
0.1639 & 0.5852 & 0.1637 & 0.5845 & 0.001175 \\
0.1401 & 0.1193 & 0.1399 & 0.1191 & 0.001497 \\
0.5933 & 0.1510 & 0.5926 & 0.1508 & 0.001244 \\
0.0655 & 0.2374 & 0.0654 & 0.2370 & 0.001536 \\
0.2984 & 0.1360 & 0.2980 & 0.1358 & 0.001407 \\
0.0648 & 0.4554 & 0.0647 & 0.4548 & 0.001364 \\
0.0625 & 0.5589 & 0.0624 & 0.5582 & 0.001269 \\
\hline${ }^{a} x_{2}{ }^{\left({ }^{s}\right)}$ initial mole fraction of component 2 in the ternary solvent mixture, $x_{4}{ }^{(\mathrm{s})}$ : \\
initial mole fraction of component 4 in the ternary solvent mixture; $x_{1}:$ mole \\
fraction solubility of the solute; $x_{2}:$ mole fraction of component 2 in the \\
quaternary solution; $x_{4}:$ mole fraction of component 4 in the quaternary \\
solution. & & & & \\
& & & &
\end{tabular}

\section{Auxiliary Information}

\section{Method/Apparatus/Procedure:}

Constant-temperature bath, calorimetric thermometer, and an ultraviolet/ visible spectrophotometer.

Ternary solvent mixtures were prepared by mass. Excess solute and solvent were placed in amber glass bottles and allowed to equilibrate for several days at constant temperature. Attainment of equilibrium was verified by several repetitive measurements and by approaching equilibrium from supersaturation. Aliquots of saturated solutions were transferred through a coarse filter into volumetric flasks, weighed and diluted with methanol. Molar concentrations were determined by spectrophotometric measurements at $356 \mathrm{~nm}$.

Source and Purity of Chemicals:

(1) $99+\%$, Acros Chemical Company, USA, recrystallized three times from acetone.

(2) $99.7 \%$, HPLC grade, Aldrich Chemical Company, Milwaukee, WI, USA.

(3) $99.9 \%$, HPLC grade, Aldrich Chemical Company.

(4) $99.8 \%$, HPLC grade, Aldrich Chemical Company.

Components 2-4 were stored over molecular sieves and distilled shortly before use.

\section{Estimated Error:}

Temperature: $\pm 0.1 \mathrm{~K}$.

$x_{2}{ }^{(s)}: \pm 0.0001$.

$x_{4}{ }^{(\mathrm{s})}: \pm 0.0001$.

$x_{1}: \pm 1.5 \%$ (relative error).

\section{Components:}

(1) Anthracene; $\mathrm{C}_{14} \mathrm{H}_{10}$; [120-12-7]

(2) 2,2,4-Trimethylpentane; $\mathrm{C}_{8} \mathrm{H}_{18}$;

[540-84-1]

(3) Cyclohexane; $\mathrm{C}_{6} \mathrm{H}_{12} ;[110-82-7]$

(4) 2-Butanol; $\mathrm{C}_{4} \mathrm{H}_{10} \mathrm{O}$; [78-92-2]

\begin{tabular}{ll}
\hline Variables: & Prepared by: \\
$T / \mathrm{K}=298.15$; Solvent Composition & W. E. Acree, Jr. \\
\hline
\end{tabular}

Experimental Values

Solubility of anthracene in 2,2,4-trimethylpentane + cyclohexane + 2-butanol mixtures $^{\mathrm{a}}$

\begin{tabular}{|c|c|c|c|c|}
\hline$x_{2}{ }^{(\mathrm{s})}$ & $x_{4}{ }^{(\mathrm{s})}$ & $x_{2}$ & $x_{4}$ & $x_{1}$ \\
\hline 0.0000 & 0.0000 & 0.0000 & 0.0000 & 0.001553 \\
\hline 1.0000 & 0.0000 & 0.9989 & 0.0000 & 0.001074 \\
\hline 0.0000 & 1.0000 & 0.0000 & 0.9994 & 0.000585 \\
\hline 0.2428 & 0.4142 & 0.2425 & 0.4137 & 0.001242 \\
\hline 0.5841 & 0.2231 & 0.5834 & 0.2228 & 0.001198 \\
\hline 0.1715 & 0.3036 & 0.1713 & 0.3032 & 0.001392 \\
\hline 0.3758 & 0.3359 & 0.3753 & 0.3355 & 0.001236 \\
\hline 0.0583 & 0.7570 & 0.0582 & 0.7563 & 0.000893 \\
\hline 0.1233 & 0.7810 & 0.1232 & 0.7803 & 0.000840 \\
\hline 0.5768 & 0.2937 & 0.5781 & 0.2934 & 0.001164 \\
\hline 0.3866 & 0.1387 & 0.3861 & 0.1385 & 0.001338 \\
\hline 0.3591 & 0.5293 & 0.3587 & 0.5287 & 0.001041 \\
\hline 0.2727 & 0.6182 & 0.2724 & 0.6176 & 0.000976 \\
\hline 0.1028 & 0.1792 & 0.1026 & 0.1789 & 0.001499 \\
\hline 0.0884 & 0.7706 & 0.0883 & 0.7699 & 0.000858 \\
\hline 0.1609 & 0.5869 & 0.1607 & 0.5863 & 0.001086 \\
\hline 0.1352 & 0.1120 & 0.1350 & 0.1118 & 0.001493 \\
\hline 0.5842 & 0.1569 & 0.5835 & 0.1567 & 0.001223 \\
\hline 0.0664 & 0.2346 & 0.0663 & 0.2342 & 0.001495 \\
\hline 0.2983 & 0.1268 & 0.2979 & 0.1266 & 0.001380 \\
\hline 0.0574 & 0.4186 & 0.0573 & 0.4180 & 0.001358 \\
\hline 0.0609 & 0.5570 & 0.0608 & 0.5564 & 0.001151 \\
\hline
\end{tabular}

${ }^{\mathrm{a}} x_{2}{ }^{(\mathrm{s})}$ : initial mole fraction of component 2 in the ternary solvent mixture, $x_{4}{ }^{(\mathrm{s})}$ : initial mole fraction of component 4 in the ternary solvent mixture; $x_{1}$ : mole fraction solubility of the solute; $x_{2}$ : mole fraction of component 2 in the quaternary solution; $x_{4}$ : mole fraction of component 4 in the quaternary solution. 


\section{Auxiliary Information}

\section{Method/Apparatus/Procedure:}

Constant-temperature bath, calorimetric thermometer, and an ultraviolet/ visible spectrophotometer.

Ternary solvent mixtures were prepared by mass. Excess solute and solvent were placed in amber glass bottles and allowed to equilibrate for several days at constant temperature. Attainment of equilibrium was verified by several repetitive measurements and by approaching equilibrium from supersaturation. Aliquots of saturated solutions were transferred through a coarse filter into volumetric flasks, weighed and diluted with methanol. Molar concentrations were determined by spectrophotometric measurements at $356 \mathrm{~nm}$.

\section{Source and Purity of Chemicals:}

(1) $99+\%$, Acros Chemical Company, USA, recrystallized three times from acetone.

(2) $99.7 \%$, HPLC grade, Aldrich Chemical Company, Milwaukee, WI, USA.

(3) $99.9 \%$, HPLC grade, Aldrich Chemical Company.

(4) $99+\%$, anhydrous, Aldrich Chemical Company.

Components 2-4 were stored over molecular sieves and distilled shortly before use.

Estimated Error:

Temperature: $\pm 0.1 \mathrm{~K}$.

$x_{2}{ }^{(\mathrm{s})}: \pm 0.0001$.

$x_{4}{ }^{(\mathrm{s})}: \pm 0.0001$.

$x_{1}: \pm 1.5 \%$ (relative error)

\section{Solubility of Anthracene in Ternary Alkane + Alcohol + Alcohol Solvent Mixtures}

\subsection{Critical evaluation of experimental solubility data}

Acree and co-workers ${ }^{7-9,11,16,26,28}$ measured the solubility of anthracene in ternary heptane + alcohol + alcohol, cyclohexane + alcohol + alcohol, and 2,2,4-trimethylpentane + alcohol + alcohol solvent mixtures at $298.15 \mathrm{~K}$. Alcohol cosolvents included 1-propanol, 2-propanol, 1-butanol, 2butanol, 2-methyl-1-propanol, and 1-pentanol. There has only been a single experimental determination for the solubility of anthracene in each of the ternary solvent systems studied. The experimental values were evaluated by curve-fitting the measured solubility data for the respective sub-binary systems to the Combined NIBS/Redlich-Kister equation, and then using the ternary solvent version of the basic model, Eq. (2), to estimate the mole fraction solubility of anthracene dissolved in ternary alkane + alcohol + alcohol solvent mixtures.

The calculated $S_{23, i}$ parameters for all of the contributing binary alkane + alcohol and alcohol + alcohol solvent systems studied are summarized in Table 1, along with the average absolute deviation between the experimental and back-calculated mole fraction solubilities. The predictive ability of Eq. (2) is given in Table 4 for the 28 ternary alkane + alcohol + alcohol solvent systems. Examination of the numerical entries in the last column of Table 4 reveals that Eq. (4) does provide accurate estimates for how the measured mole fraction solubilities vary with ternary solvent composition. Average
TABLE 4. Summarized comparison between experimental solubilities of anthracene in ternary alkane + alcohol + alcohol solvent mixtures and predicted values based on Eq. (2)

\begin{tabular}{lc}
\hline \hline Ternary solvent mixture & \% Dev. \\
\hline Heptane + 1-propanol + 1-butanol & 1.30 \\
Heptane + 1-propanol + 2-butanol & 0.96 \\
Heptane + 2-propanol + 1-butanol & 1.64 \\
Heptane + 2-propanol + 2-butanol & 1.69 \\
Heptane + 1-propanol + 1-pentanol & 1.48 \\
Heptane + 2-propanol + 1-pentanol & 1.74 \\
Heptane + 1-butanol + 1-pentanol & 1.81 \\
Heptane + 2-butanol + 1-pentanol & 2.03 \\
Cyclohexane + 1-propanol + 1-butanol & 1.45 \\
Cyclohexane + 1-propanol + 2-butanol & 1.76 \\
Cyclohexane + 2-propanol + 1-butanol & 1.70 \\
Cyclohexane + 2-propanol + 2-butanol & 1.57 \\
Cyclohexane + 1-propanol + 1-pentanol & 1.17 \\
Cyclohexane + 2-propanol + 1-pentanol & 1.10 \\
Cyclohexane + 1-butanol + 1-pentanol & 1.25 \\
Cyclohexane + 2-butanol + 1-pentanol & 1.29 \\
Cyclohexane + 1-propanol + 2-methyl-1-propanol & 1.88 \\
Cyclohexane + 2-propanol + 2-methyl-1-propanol & 1.21 \\
Cyclohexane + 1-butanol + 2-methyl-1-propanol & 0.97 \\
Cyclohexane + 2-butanol + 2-methyl-1-propanol & 1.09 \\
2,2,4-Trimethylpentane + 1-propanol + 1-butanol & 0.77 \\
2,2,4-Trimethylpentane + 1-propanol + 2-butanol & 0.52 \\
2,2,4-Trimethylpentane + 2-propanol + 1-butanol & 1.11 \\
2,2,4-Trimethylpentane + 2-propanol + 2-butanol & 1.27 \\
2,2,4-Trimethylpentane + 1-propanol + 1-pentanol & 1.31 \\
2,2,4-Trimethylpentane + 2-propanol + 1-pentanol & 1.30 \\
2,2,4-Trimethylpentane + 1-butanol + 1-pentanol & 1.74 \\
2,2,4-Trimethylpentane + 2-butanol + 1-pentanol & 1.56 \\
\hline a Dev (\%) = (100/ $N$ ) $\Sigma\left|\left[x_{1}{ }^{\text {exp } ~-~} x_{1}{ }^{\text {cal }}\right] / x_{1}{ }^{\text {exp }}\right|$. & \\
&
\end{tabular}

percent deviations range from $0.5 \%$ to $2.0 \%$, suggesting that there are no obvious outliers in an individual ternary solvent data set. None of the experimental data points was flagged as an outlier.

The experimental anthracene solubility data for ternary heptane + alcohol + alcohol, cyclohexane + alcohol + alcohol, and 2,2,4-trimethylpentane + alcohol + alcohol solvent mixtures are tabulated in Secs. 3.2-3.4.

\subsection{Anthracene solubility data in ternary heptane + alcohol + alcohol solvent mixtures}

\begin{tabular}{ll}
\hline \hline Components: & Original Measurements: \\
(1) Anthracene; $\mathrm{C}_{14} \mathrm{H}_{10} ;[120-12-7]$ & ${ }^{16}$ T. Deng, S. D. Childress, K. M. \\
(2) 1-Propanol; $\mathrm{C}_{3} \mathrm{H}_{8} \mathrm{O} ;[71-23-8]$ & De Fina, and W. E. Acree, Jr., \\
(3) 1-Butanol; $\mathrm{C}_{4} \mathrm{H}_{10} \mathrm{O} ;[71-36-3]$ & Chem. Eng. Commun. 172, 217 \\
(4) Heptane; $\mathrm{C}_{7} \mathrm{H}_{16} ;[142-82-5]$ & $(1999)$. \\
\hline Variables: & Prepared by: \\
$T / \mathrm{K}=298.15 ;$ Solvent Composition & W. E. Acree, Jr. \\
\hline
\end{tabular}


Experimental Values

Solubility of anthracene in 1-propanol +1 -butanol + heptane mixtures ${ }^{\mathrm{a}}$

\begin{tabular}{lcccc}
\hline \hline$x_{2}{ }^{(\mathrm{s})}$ & $x_{3}{ }^{(\mathrm{s})}$ & $x_{2}$ & $x_{3}$ & $x_{1}$ \\
\hline 0.0000 & 0.0000 & 0.0000 & 0.0000 & 0.001571 \\
1.0000 & 0.0000 & 0.9994 & 0.0000 & 0.000591 \\
0.0000 & 1.0000 & 0.0000 & 0.9992 & 0.000801 \\
0.4340 & 0.3473 & 0.4336 & 0.3470 & 0.000992 \\
0.1911 & 0.7121 & 0.1909 & 0.7115 & 0.000897 \\
0.3574 & 0.2855 & 0.3569 & 0.2852 & 0.001163 \\
0.3223 & 0.5161 & 0.3120 & 0.5156 & 0.000935 \\
0.7909 & 0.0929 & 0.7903 & 0.0928 & 0.000796 \\
0.7651 & 0.1790 & 0.7645 & 0.1789 & 0.000723 \\
0.2440 & 0.6922 & 0.2438 & 0.6916 & 0.000842 \\
0.1441 & 0.5718 & 0.1439 & 0.5712 & 0.001113 \\
0.4640 & 0.4749 & 0.4636 & 0.4745 & 0.000787 \\
0.5695 & 0.3704 & 0.5691 & 0.3701 & 0.000757 \\
0.2428 & 0.1953 & 0.2425 & 0.1950 & 0.001388 \\
0.7797 & 0.1340 & 0.7791 & 0.1339 & 0.000741 \\
0.6040 & 0.2435 & 0.6035 & 0.2433 & 0.000838 \\
0.1678 & 0.2630 & 0.1676 & 0.2626 & 0.001390 \\
0.1308 & 0.7371 & 0.1307 & 0.7364 & 0.000952 \\
0.3105 & 0.1336 & 0.3101 & 0.1334 & 0.001364 \\
0.1485 & 0.4795 & 0.1483 & 0.4778 & 0.001222 \\
0.5422 & 0.1114 & 0.5416 & 0.1113 & 0.001101 \\
0.6347 & 0.1042 & 0.6335 & 0.1041 & 0.000988 \\
\hline
\end{tabular}

${ }^{\mathrm{a}} x_{2}{ }^{(\mathrm{s})}$ : initial mole fraction of component 2 in the ternary solvent mixture, $x_{3}{ }^{(\mathrm{s})}$ : initial mole fraction of component 3 in the ternary solvent mixture; $x_{1}$ : mole fraction solubility of the solute; $x_{2}$ : mole fraction of component 2 in the quaternary solution; $x_{3}$ : mole fraction of component 3 in the quaternary solution.

\section{Auxiliary Information}

\section{Method/Apparatus/Procedure:}

Constant-temperature bath, calorimetric thermometer, and an ultraviolet/ visible spectrophotometer.

Ternary solvent mixtures were prepared by mass. Excess solute and solvent were placed in amber glass bottles and allowed to equilibrate for several days at constant temperature. Attainment of equilibrium was verified by several repetitive measurements and by approaching equilibrium from supersaturation. Aliquots of saturated solutions were transferred through a coarse filter into volumetric flasks, weighed and diluted with methanol. Molar concentrations were determined by spectrophotometric measurements at $356 \mathrm{~nm}$.

\section{Source and Purity of Chemicals:}

(1) $99+\%$, Acros Chemical Company, USA, recrystallized three times from acetone.

(2) $99+\%$, anhydrous, Aldrich Chemical Company, Milwaukee, WI, USA.

(3) $99.8 \%$, HPLC grade, Aldrich Chemical Company.

(4) $99+\%$, HPLC grade, Aldrich Chemical Company.

Components 2-4 were stored over molecular sieves and distilled shortly before use.

Estimated Error:

Temperature: $\pm 0.1 \mathrm{~K}$.

$x_{2}{ }^{(\mathrm{s})}: \pm 0.0001$.

$x_{3}{ }^{(\mathrm{s})}: \pm 0.0001$

$x_{1}: \pm 1.5 \%$ (relative error)

\section{Components:}

(1) Anthracene; $\mathrm{C}_{14} \mathrm{H}_{10} ;[120-12-7]$

(2) 1-Propanol; $\mathrm{C}_{3} \mathrm{H}_{8} \mathrm{O}$; [71-23-8]

(3) 2-Butanol; $\mathrm{C}_{4} \mathrm{H}_{10} \mathrm{O}$; [78-92-2]

(4) Heptane; $\mathrm{C}_{7} \mathrm{H}_{16} ;[142-82-5]$

\section{Original Measurements:}

${ }^{16}$ T. Deng, S. D. Childress, K. M. De Fina, and W. E. Acree, Jr., Chem. Eng. Commun. 172, 217 (1999).
Variables:

Prepared by:

$T / \mathrm{K}=298.15$; Solvent Composition $\quad$ W. E. Acree, Jr.

\section{Experimental Values}

Solubility of anthracene in 1-propanol +2 -butanol + heptane mixtures ${ }^{\mathrm{a}}$

\begin{tabular}{|c|c|c|c|c|}
\hline$x_{2}{ }^{(\mathrm{s})}$ & $x_{3}{ }^{(\mathrm{s})}$ & $x_{2}$ & $x_{3}$ & $x_{1}$ \\
\hline 0.0000 & 0.0000 & 0.0000 & 0.0000 & 0.001571 \\
\hline 1.0000 & 0.0000 & 0.9994 & 0.0000 & 0.000591 \\
\hline 0.0000 & 1.0000 & 0.0000 & 0.9994 & 0.000585 \\
\hline 0.4326 & 0.3478 & 0.4322 & 0.3475 & 0.000900 \\
\hline 0.1906 & 0.7123 & 0.1905 & 0.7118 & 0.000709 \\
\hline 0.3557 & 0.2863 & 0.3553 & 0.2860 & 0.001087 \\
\hline 0.3201 & 0.5169 & 0.3198 & 0.5165 & 0.000807 \\
\hline 0.7924 & 0.0930 & 0.7918 & 0.0929 & 0.000763 \\
\hline 0.7655 & 0.1785 & 0.7650 & 0.1784 & 0.000666 \\
\hline 0.2474 & 0.6916 & 0.2472 & 0.6911 & 0.000672 \\
\hline 0.1438 & 0.5713 & 0.1437 & 0.5707 & 0.001004 \\
\hline 0.4695 & 0.4707 & 0.4692 & 0.4704 & 0.000655 \\
\hline 0.5705 & 0.3712 & 0.5701 & 0.3710 & 0.000659 \\
\hline 0.2408 & 0.1950 & 0.2405 & 0.1947 & 0.001326 \\
\hline 0.7783 & 0.1357 & 0.7777 & 0.1356 & 0.000713 \\
\hline 0.6020 & 0.2440 & 0.6015 & 0.2438 & 0.000805 \\
\hline 0.1623 & 0.2633 & 0.1621 & 0.2630 & 0.001325 \\
\hline 0.1351 & 0.7343 & 0.1350 & 0.7337 & 0.000795 \\
\hline 0.3158 & 0.1255 & 0.3155 & 0.1253 & 0.001337 \\
\hline 0.1541 & 0.4724 & 0.1539 & 0.4719 & 0.001136 \\
\hline 0.5464 & 0.1069 & 0.5458 & 0.1068 & 0.001068 \\
\hline 0.6359 & 0.1052 & 0.6353 & 0.1051 & 0.000957 \\
\hline
\end{tabular}

${ }^{\mathrm{a}} x_{2}{ }^{(\mathrm{s})}$ : initial mole fraction of component 2 in the ternary solvent mixture, $x_{3}{ }^{(\mathrm{s})}$ : initial mole fraction of component 3 in the ternary solvent mixture; $x_{1}$ : mole fraction solubility of the solute; $x_{2}$ : mole fraction of component 2 in the quaternary solution; $x_{3}$ : mole fraction of component 3 in the quaternary solution.

\section{Auxiliary Information}

\section{Method/Apparatus/Procedure:}

Constant-temperature bath, calorimetric thermometer, and an ultraviolet/ visible spectrophotometer.

Ternary solvent mixtures were prepared by mass. Excess solute and solvent were placed in amber glass bottles and allowed to equilibrate for several days at constant temperature. Attainment of equilibrium was verified by several repetitive measurements and by approaching equilibrium from supersaturation. Aliquots of saturated solutions were transferred through a coarse filter into volumetric flasks, weighed and diluted with methanol. Molar concentrations were determined by spectrophotometric measurements at $356 \mathrm{~nm}$.

\section{Source and Purity of Chemicals:}

(1) $99+\%$, Acros Chemical Company, USA, recrystallized three times from acetone.

(2) $99+\%$, anhydrous, Aldrich Chemical Company, Milwaukee, WI, USA.

(3) $99+\%$, anhydrous, Aldrich Chemical Company.

(4) $99+\%$, HPLC grade, Aldrich Chemical Company.

Components 2-4 were stored over molecular sieves and distilled shortly before use.

\section{Estimated Error:}

Temperature: $\pm 0.1 \mathrm{~K}$.

$x_{2}{ }^{(\mathrm{s})}: \pm 0.0001$.

$x_{3}{ }^{(\mathrm{s})}: \pm 0.0001$.

$x_{1}: \pm 1.5 \%$ (relative error). 


\section{Components:}

(1) Anthracene; $\mathrm{C}_{14} \mathrm{H}_{10} ;[120-12-7]$

(2) 2-Propanol; $\mathrm{C}_{3} \mathrm{H}_{8} \mathrm{O} ;[67-63-0]$

(3) 1-Butanol; $\mathrm{C}_{4} \mathrm{H}_{10} \mathrm{O} ;[$ [71-36-3]

(4) Heptane; $\mathrm{C}_{7} \mathrm{H}_{16} ;[142-82-5]$

\begin{tabular}{ll}
\hline Variables: & Prepared by: \\
$T / \mathrm{K}=298.15 ;$ Solvent Composition & W. E. Acree, Jr. \\
\hline
\end{tabular}

Experimental Values

Solubility of anthracene in 2-propanol +1 -butanol + heptane mixtures ${ }^{\mathrm{a}}$

\begin{tabular}{lcccc}
\hline \hline$x_{2}{ }^{(\mathrm{s})}$ & $x_{3}{ }^{(\mathrm{s})}$ & $x_{2}$ & $x_{3}$ & $x_{1}$ \\
\hline 0.0000 & 0.0000 & 0.0000 & 0.0000 & 0.001571 \\
1.0000 & 0.0000 & 0.9996 & 0.0000 & 0.000411 \\
0.0000 & 1.0000 & 0.0000 & 0.9992 & 0.000801 \\
0.4261 & 0.3522 & 0.4257 & 0.3519 & 0.000917 \\
0.1938 & 0.7099 & 0.1936 & 0.7093 & 0.000854 \\
0.3507 & 0.2866 & 0.3503 & 0.2863 & 0.001093 \\
0.3102 & 0.5257 & 0.3099 & 0.5252 & 0.000876 \\
0.7882 & 0.0928 & 0.7877 & 0.0927 & 0.000651 \\
0.7565 & 0.1864 & 0.7561 & 0.1863 & 0.000584 \\
0.2363 & 0.7018 & 0.2361 & 0.7012 & 0.000821 \\
0.1381 & 0.5762 & 0.1379 & 0.5756 & 0.001109 \\
0.4538 & 0.4826 & 0.4535 & 0.4823 & 0.000705 \\
0.5661 & 0.3757 & 0.5657 & 0.3755 & 0.000652 \\
0.2417 & 0.2022 & 0.2414 & 0.2019 & 0.001330 \\
0.7647 & 0.1423 & 0.7642 & 0.1422 & 0.000625 \\
0.5694 & 0.2473 & 0.5690 & 0.2471 & 0.000787 \\
0.1658 & 0.2631 & 0.1656 & 0.2627 & 0.001373 \\
0.1269 & 0.7442 & 0.1268 & 0.7435 & 0.000904 \\
0.3082 & 0.1340 & 0.3078 & 0.1338 & 0.001308 \\
0.1432 & 0.4853 & 0.1430 & 0.4847 & 0.001233 \\
0.5336 & 0.1201 & 0.5331 & 0.1200 & 0.001022 \\
0.6264 & 0.1090 & 0.6258 & 0.1089 & 0.000881 \\
\hline
\end{tabular}

${ }^{\mathrm{a}} x_{2}{ }^{(\mathrm{s})}$ : initial mole fraction of component 2 in the ternary solvent mixture, $x_{3}{ }^{(\mathrm{s})}$ initial mole fraction of component 3 in the ternary solvent mixture; $x_{1}$ : mole fraction solubility of the solute; $x_{2}$ : mole fraction of component 2 in the quaternary solution; $x_{3}$ : mole fraction of component 3 in the quaternary solution.

\section{Auxiliary Information}

\section{Method/Apparatus/Procedure:}

Constant-temperature bath, calorimetric thermometer, and an ultraviolet/ visible spectrophotometer.

Ternary solvent mixtures were prepared by mass. Excess solute and solvent were placed in amber glass bottles and allowed to equilibrate for several days at constant temperature. Attainment of equilibrium was verified by several repetitive measurements and by approaching equilibrium from supersaturation. Aliquots of saturated solutions were transferred through a coarse filter into volumetric flasks, weighed and diluted with methanol. Molar concentrations were determined by spectrophotometric measurements at $356 \mathrm{~nm}$.

\section{Source and Purity of Chemicals:}

(1) $99+\%$, Acros Chemical Company, USA, recrystallized three times from acetone.

(2) $99+\%$, anhydrous, Aldrich Chemical Company, Milwaukee, WI, USA.

(3) $99.8 \%$, HPLC grade, Aldrich Chemical Company.

(4) $99+\%$, HPLC grade, Aldrich Chemical Company.

Components 2-4 were stored over molecular sieves and distilled shortly before use.
Estimated Error:

Temperature: $\pm 0.1 \mathrm{~K}$.

$x_{2}{ }^{(\mathrm{s})}: \pm 0.0001$.

$x_{3}{ }^{(\mathrm{s})}: \pm 0.0001$.

$x_{1}: \pm 1.5 \%$ (relative error).

\begin{tabular}{ll}
\hline \hline Components: & Original Measurements: \\
(1) Anthracene; $\mathrm{C}_{14} \mathrm{H}_{10} ;[120-12-7]$ & ${ }^{16}$ T. Deng, S. D. Childress, K. M. \\
(2) 2-Propanol; $\mathrm{C}_{3} \mathrm{H}_{8} \mathrm{O} ;[67-63-0]$ & De Fina, and W. E. Acree, Jr., \\
(3) 2-Butanol; $\mathrm{C}_{4} \mathrm{H}_{10} \mathrm{O} ;[78-92-2]$ & Chem. Eng. Commun. 172, 217 \\
(4) Heptane; $\mathrm{C}_{7} \mathrm{H}_{16} ;[142-82-5]$ & $(1999)$.
\end{tabular}

\begin{tabular}{ll}
\hline Variables: & Prepared by: \\
$T / \mathrm{K}=298.15 ;$ Solvent Composition & W. E. Acree, Jr. \\
\hline
\end{tabular}

Experimental Values

Solubility of anthracene in 2-propanol +2 -butanol + heptane mixtures ${ }^{\mathrm{a}}$

\begin{tabular}{lcccc}
\hline \hline$x_{2}{ }^{(\mathrm{s})}$ & $x_{3}{ }^{(\mathrm{s})}$ & $x_{2}$ & $x_{3}$ & $x_{1}$ \\
\hline 0.0000 & 0.0000 & 0.0000 & 0.0000 & 0.001571 \\
1.0000 & 0.0000 & 0.9996 & 0.0000 & 0.000411 \\
0.0000 & 1.0000 & 0.0000 & 0.9994 & 0.000585 \\
0.4280 & 0.3485 & 0.4276 & 0.3482 & 0.000844 \\
0.1882 & 0.7142 & 0.1881 & 0.7137 & 0.000713 \\
0.3522 & 0.2867 & 0.3518 & 0.2864 & 0.001044 \\
0.3155 & 0.5211 & 0.3153 & 0.5207 & 0.000778 \\
0.7870 & 0.0972 & 0.7865 & 0.0971 & 0.000625 \\
0.7600 & 0.1831 & 0.7596 & 0.1830 & 0.000544 \\
0.2383 & 0.6976 & 0.2381 & 0.6972 & 0.000644 \\
0.1401 & 0.5754 & 0.1400 & 0.5748 & 0.001000 \\
0.4600 & 0.4805 & 0.4597 & 0.4802 & 0.000593 \\
0.5624 & 0.3775 & 0.5621 & 0.3773 & 0.000580 \\
0.2392 & 0.1938 & 0.2389 & 0.1935 & 0.001302 \\
0.7748 & 0.1368 & 0.7743 & 0.1367 & 0.000589 \\
0.5982 & 0.2461 & 0.5974 & 0.2459 & 0.000713 \\
0.1604 & 0.2622 & 0.1602 & 0.2619 & 0.001330 \\
0.1303 & 0.7370 & 0.1302 & 0.7364 & 0.000767 \\
0.3044 & 0.1317 & 0.3040 & 0.1315 & 0.001290 \\
0.1474 & 0.4795 & 0.1472 & 0.4790 & 0.001127 \\
0.5362 & 0.1152 & 0.5357 & 0.1151 & 0.000987 \\
0.6248 & 0.1106 & 0.6243 & 0.1105 & 0.000866 \\
\hline${ }^{a} x_{2}^{(\mathrm{s})}:$ initial mole fraction of component 2 in the ternary solvent mixture, $x_{3}^{(\mathrm{s})}$ : \\
initial mole fraction of component 3 in the ternary solvent mixture; $x_{1}:$ mole \\
fraction solubility of the solute; $x_{2}:$ mole fraction of component 2 in the \\
quaternary solution; $x_{3}:$ mole fraction of component 3 in the quaternary \\
solution. & & & & \\
& & & &
\end{tabular}

Auxiliary Information

Method/Apparatus/Procedure:

Constant-temperature bath, calorimetric thermometer, and an ultraviolet/ visible spectrophotometer.

Ternary solvent mixtures were prepared by mass. Excess solute and solvent were placed in amber glass bottles and allowed to equilibrate for several days at constant temperature. Attainment of equilibrium was verified by several repetitive measurements and by approaching equilibrium from supersaturation. Aliquots of saturated solutions were transferred through a coarse filter into volumetric flasks, weighed and diluted with methanol. Molar concentrations were determined by spectrophotometric measurements at $356 \mathrm{~nm}$. 
Source and Purity of Chemicals:

(1) $99+\%$, Acros Chemical Company, USA, recrystallized three times from acetone.

(2) $99+\%$, anhydrous, Aldrich Chemical Company, Milwaukee, WI, USA.

(3) $99+\%$, anhydrous, Aldrich Chemical Company.

(4) $99+\%$, HPLC grade, Aldrich Chemical Company.

Components 2-4 were stored over molecular sieves and distilled shortly before use.

Estimated Error:

Temperature: $\pm 0.1 \mathrm{~K}$.

$x_{2}{ }^{(\mathrm{s})}: \pm 0.0001$.

$x_{3}{ }^{(\mathrm{s})}: \pm 0.0001$

$x_{1}: \pm 1.5 \%$ (relative error).

\begin{tabular}{ll}
\hline \hline Components: & $\begin{array}{l}\text { Original Measurements: } \\
\text { (1) Anthracene; } \mathrm{C}_{14} \mathrm{H}_{10} ;[120-12-7]\end{array}$ \\
${ }^{8}$ A. Proctor, B. A. Martine, and \\
(2) Heptane; $\mathrm{C}_{7} \mathrm{H}_{16} ;[142-82-5]$ & W. E. Acree, Jr., J. Chem. Eng. \\
(3) 1-Propanol; $\mathrm{C}_{3} \mathrm{H}_{8} \mathrm{O} ;[71-23-8]$ & Data 53, 2197 (2008). \\
(4) 1-Pentanol; $\mathrm{C}_{5} \mathrm{H}_{12} \mathrm{O} ;[71-41-0]$ & \\
\hline Variables: & Prepared by: \\
$T / \mathrm{K}=298.15 ;$ Solvent Composition & W. E. Acree, Jr. \\
\hline
\end{tabular}

Experimental Values

Solubility of anthracene in heptane +1 -propanol +1 -pentanol mixtures ${ }^{\mathrm{a}}$

\begin{tabular}{lcccc}
\hline \hline$x_{2}{ }^{(\mathrm{s})}$ & $x_{3}{ }^{(\mathrm{s})}$ & $x_{2}$ & $x_{3}$ & $x_{1}$ \\
\hline 0.0000 & 0.0000 & 0.0000 & 0.0000 & 0.001097 \\
1.0000 & 0.0000 & 0.9984 & 0.0000 & 0.001571 \\
0.0000 & 1.0000 & 0.0000 & 0.9994 & 0.000591 \\
0.0555 & 0.7846 & 0.0555 & 0.7840 & 0.000771 \\
0.0645 & 0.4982 & 0.0644 & 0.4977 & 0.000932 \\
0.0652 & 0.2894 & 0.0651 & 0.2891 & 0.001038 \\
0.0913 & 0.7920 & 0.0912 & 0.7914 & 0.000804 \\
0.0706 & 0.5966 & 0.0705 & 0.5961 & 0.000891 \\
0.1136 & 0.2229 & 0.1135 & 0.2227 & 0.001107 \\
0.1138 & 0.8121 & 0.1137 & 0.8114 & 0.000803 \\
0.1464 & 0.1478 & 0.1462 & 0.1477 & 0.001192 \\
0.1578 & 0.6208 & 0.1577 & 0.6202 & 0.000949 \\
0.1778 & 0.3425 & 0.1776 & 0.3421 & 0.001114 \\
0.2329 & 0.4449 & 0.2326 & 0.4444 & 0.001117 \\
0.2662 & 0.6497 & 0.2659 & 0.6490 & 0.001031 \\
0.3035 & 0.1575 & 0.3031 & 0.1573 & 0.001326 \\
0.3502 & 0.5383 & 0.3498 & 0.5377 & 0.001168 \\
0.3684 & 0.3654 & 0.3679 & 0.3649 & 0.001273 \\
0.3976 & 0.1625 & 0.3970 & 0.1623 & 0.001403 \\
0.5675 & 0.3218 & 0.5667 & 0.3213 & 0.001441 \\
0.5788 & 0.2271 & 0.5779 & 0.2268 & 0.001483 \\
0.5790 & 0.1835 & 0.5781 & 0.1832 & 0.001507 \\
\hline${ }^{a} x_{2}{ }^{(s)}:$ initial mole fraction of component 2 in the ternary solvent mixture, $x_{3}{ }^{(\mathrm{s})}:$ \\
initial mole fraction of component 3 in the ternary solvent mixture; $x_{1}:$ mole \\
fraction solubility of the solute; $x_{2}:$ mole fraction of component 2 in the \\
quaternary solution $; x_{3}:$ mole fraction of component 3 in the quaternary \\
solution. & & & & \\
& & & &
\end{tabular}

\section{Auxiliary Information}

\section{Method/Apparatus/Procedure:}

Constant-temperature bath, calorimetric thermometer, and an ultraviolet/ visible spectrophotometer.
Ternary solvent mixtures were prepared by mass. Excess solute and solvent were placed in amber glass bottles and allowed to equilibrate for several days at constant temperature. Attainment of equilibrium was verified by several repetitive measurements and by approaching equilibrium from supersaturation. Aliquots of saturated solutions were transferred through a coarse filter into volumetric flasks, weighed and diluted with methanol. Molar concentrations were determined by spectrophotometric measurements at $356 \mathrm{~nm}$.

\section{Source and Purity of Chemicals:}

(1) $99+\%$, Aldrich Chemical Company, Milwaukee, Wisconsin, USA, was recrystallized several times from acetone.

(2) $99 \%$, anhydrous, Aldrich Chemical Company, Milwaukee, WI, USA.

(3) $99+\%$, anhydrous, Aldrich Chemical Company.

(4) $99 \%$, Aldrich Chemical Company.

Components $2-4$ were stored over molecular sieves and distilled shortly before use.

\section{Estimated Error:}

Temperature: $\pm 0.05 \mathrm{~K}$

$x_{2}{ }^{(\mathrm{s})}: \pm 0.0001$.

$x_{3}{ }^{(\mathrm{s})}: \pm 0.0001$.

$x_{1}: \pm 1.5 \%$ (relative error).

\begin{tabular}{ll}
\hline \hline Components: & Original Measurements: \\
(1) Anthracene; $\mathrm{C}_{14} \mathrm{H}_{10} ;[120-12-7]$ & ${ }^{8}$ A. Proctor, B. A. Martine, and \\
(2) Heptane; $\mathrm{C}_{7} \mathrm{H}_{16} ;[142-82-5]$ & W. E. Acree, Jr., J. Chem. Eng. \\
(3) 2-Propanol; $\mathrm{C}_{3} \mathrm{H}_{8} \mathrm{O} ;[67-63-0]$ & Data 53, 2197 (2008). \\
(4) 1-Pentanol; $\mathrm{C}_{5} \mathrm{H}_{12} \mathrm{O} ;[71-41-0]$ & \\
\hline Variables: & Prepared by: \\
$T / \mathrm{K}=298.15 ;$ Solvent Composition & W. E. Acree, Jr. \\
\hline
\end{tabular}

Experimental Values

Solubility of anthracene in heptane +2 -propanol +1 -pentanol mixtures ${ }^{\mathrm{a}}$

\begin{tabular}{lcccc}
\hline \hline$x_{2}{ }^{(\mathrm{s})}$ & $x_{3}{ }^{(\mathrm{s})}$ & $x_{2}$ & $x_{3}$ & $x_{1}$ \\
\hline 0.0000 & 0.0000 & 0.0000 & 0.0000 & 0.001097 \\
1.0000 & 0.0000 & 0.9984 & 0.0000 & 0.001571 \\
0.0000 & 1.0000 & 0.0000 & 0.9996 & 0.000411 \\
0.0620 & 0.5132 & 0.0619 & 0.5128 & 0.000817 \\
0.0642 & 0.6030 & 0.0642 & 0.6025 & 0.000759 \\
0.0671 & 0.2616 & 0.0670 & 0.2613 & 0.001001 \\
0.0681 & 0.7774 & 0.0681 & 0.7769 & 0.000643 \\
0.0894 & 0.7770 & 0.0893 & 0.7765 & 0.000666 \\
0.1057 & 0.2210 & 0.1056 & 0.2208 & 0.001044 \\
0.1124 & 0.8117 & 0.1123 & 0.8112 & 0.000666 \\
0.1451 & 0.1773 & 0.1449 & 0.1771 & 0.001144 \\
0.1647 & 0.6083 & 0.1646 & 0.6078 & 0.000852 \\
0.1832 & 0.3400 & 0.1830 & 0.3396 & 0.001071 \\
0.2299 & 0.4592 & 0.2297 & 0.4587 & 0.001027 \\
0.2615 & 0.6233 & 0.2613 & 0.6227 & 0.000937 \\
0.3117 & 0.1651 & 0.3113 & 0.1649 & 0.001307 \\
0.3591 & 0.5338 & 0.3587 & 0.3583 & 0.001085 \\
0.3742 & 0.3521 & 0.3738 & 0.3517 & 0.001222 \\
0.4095 & 0.1416 & 0.4089 & 0.1414 & 0.001403 \\
0.5784 & 0.3035 & 0.5776 & 0.3031 & 0.001383 \\
0.5975 & 0.2349 & 0.5966 & 0.2346 & 0.001438 \\
0.6010 & 0.1596 & 0.6000 & 0.1594 & 0.001526 \\
\hline
\end{tabular}

${ }^{\mathrm{a}} x_{2}{ }^{(\mathrm{s})}$ : initial mole fraction of component 2 in the ternary solvent mixture, $x_{3}{ }^{(\mathrm{s})}$ : initial mole fraction of component 3 in the ternary solvent mixture; $x_{1}$ : mole fraction solubility of the solute; $x_{2}$ : mole fraction of component 2 in the quaternary solution; $x_{3}$ : mole fraction of component 3 in the quaternary solution. 


\section{Auxiliary Information}

Method/Apparatus/Procedure:

Constant-temperature bath, calorimetric thermometer, and an ultraviolet/ visible spectrophotometer.

Ternary solvent mixtures were prepared by mass. Excess solute and solvent were placed in amber glass bottles and allowed to equilibrate for several days at constant temperature. Attainment of equilibrium was verified by several repetitive measurements and by approaching equilibrium from supersaturation. Aliquots of saturated solutions were transferred through a coarse filter into volumetric flasks, weighed and diluted with methanol. Molar concentrations were determined by spectrophotometric measurements at $356 \mathrm{~nm}$.

\section{Source and Purity of Chemicals:}

(1) $99+\%$, Aldrich Chemical Company, Milwaukee, WI, USA, was recrystallized several times from acetone.

(2) $99 \%$, anhydrous, Aldrich Chemical Company, Milwaukee, WI, USA.

(3) $99+\%$, anhydrous, Aldrich Chemical Company

(4) $99 \%$, Aldrich Chemical Company.

Components 2-4 were stored over molecular sieves and distilled shortly before use.

\section{Estimated Error:}

Temperature: $\pm 0.05 \mathrm{~K}$.

$x_{2}{ }^{(\mathrm{s})}: \pm 0.0001$.

$x_{3}{ }^{(\mathrm{s})}: \pm 0.0001$.

$x_{1}: \pm 1.5 \%$ (relative error).

\section{Components:}

(1) Anthracene; $\mathrm{C}_{14} \mathrm{H}_{10} ;[120-12-7]$

(2) Heptane; $\mathrm{C}_{7} \mathrm{H}_{16} ;[142-82-5]$

(3) 1-Butanol; $\mathrm{C}_{4} \mathrm{H}_{10} \mathrm{O} ;[71-36-3]$

(4) 1-Pentanol; $\mathrm{C}_{5} \mathrm{H}_{12} \mathrm{O}$; [71-41-0]

\begin{tabular}{ll}
\hline Variables: & Prepared by: \\
$T / \mathrm{K}=298.15 ;$ Solvent Composition & W. E. Acree, Jr. \\
\hline
\end{tabular}

Experimental Values

Solubility of anthracene in heptane +1 -butanol +1 -pentanol mixtures ${ }^{\mathrm{a}}$

\begin{tabular}{lcccc}
\hline \hline$x_{2}{ }^{(\mathrm{s})}$ & $x_{3}{ }^{(\mathrm{s})}$ & $x_{2}$ & $x_{3}$ & $x_{1}$ \\
\hline 0.0000 & 0.0000 & 0.0000 & 0.0000 & 0.001097 \\
1.0000 & 0.0000 & 0.9984 & 0.0000 & 0.001571 \\
0.0000 & 1.0000 & 0.0000 & 0.9992 & 0.000801 \\
0.0686 & 0.7538 & 0.0685 & 0.7531 & 0.000967 \\
0.0777 & 0.5553 & 0.0776 & 0.5547 & 0.001020 \\
0.0794 & 0.4373 & 0.0793 & 0.4368 & 0.001063 \\
0.0839 & 0.2295 & 0.0838 & 0.2292 & 0.001129 \\
0.0942 & 0.7642 & 0.0941 & 0.7634 & 0.000982 \\
0.1017 & 0.1968 & 0.1016 & 0.1966 & 0.001168 \\
0.1389 & 0.7520 & 0.1388 & 0.7512 & 0.001014 \\
0.1647 & 0.1185 & 0.1645 & 0.1184 & 0.001234 \\
0.1792 & 0.3186 & 0.1790 & 0.3182 & 0.001194 \\
0.1796 & 0.5751 & 0.1794 & 0.5745 & 0.001114 \\
0.2548 & 0.3944 & 0.2545 & 0.3939 & 0.001247 \\
0.2992 & 0.5828 & 0.2988 & 0.5821 & 0.001221
\end{tabular}

\begin{tabular}{|c|c|c|c|c|}
\hline 0.3299 & 0.1225 & 0.3294 & 0.1223 & 0.001372 \\
\hline 0.3826 & 0.4895 & 0.3821 & 0.4889 & 0.001315 \\
\hline 0.3931 & 0.3375 & 0.3926 & 0.3370 & 0.001365 \\
\hline 0.4032 & 0.1428 & 0.4026 & 0.1426 & 0.001403 \\
\hline 0.6023 & 0.2682 & 0.6014 & 0.2678 & 0.001530 \\
\hline 0.6052 & 0.1952 & 0.6043 & 0.1949 & 0.001534 \\
\hline 0.6126 & 0.1489 & 0.6117 & 0.1487 & 0.001507 \\
\hline
\end{tabular}

\section{Auxiliary Information}

\begin{abstract}
Method/Apparatus/Procedure:
Constant-temperature bath, calorimetric thermometer, and an ultraviolet/ visible spectrophotometer.

Ternary solvent mixtures were prepared by mass. Excess solute and solvent were placed in amber glass bottles and allowed to equilibrate for several days at constant temperature. Attainment of equilibrium was verified by several repetitive measurements and by approaching equilibrium from supersaturation. Aliquots of saturated solutions were transferred through a coarse filter into volumetric flasks, weighed and diluted with methanol. Molar concentrations were determined by spectrophotometric measurements at $356 \mathrm{~nm}$.
\end{abstract}

\section{Source and Purity of Chemicals:}

(1) $99+\%$, Aldrich Chemical Company, Milwaukee, WI, USA, was recrystallized several times from acetone.

(2) $99 \%$, anhydrous, Aldrich Chemical Company, Milwaukee, WI, USA.

(3) $99+\%$, anhydrous, Aldrich Chemical Company.

(4) $99 \%$, Aldrich Chemical Company.

Components 2-4 were stored over molecular sieves and distilled shortly before use.

\section{Estimated Error:}

Temperature: $\pm 0.05 \mathrm{~K}$

$x_{2}{ }^{(\mathrm{s})}: \pm 0.0001$.

$x_{3}{ }^{(\mathrm{s})}: \pm 0.0001$.

$x_{1}: \pm 1.5 \%$ (relative error).

\begin{tabular}{|c|c|}
\hline $\begin{array}{l}\text { Components: } \\
\text { (1) Anthracene; } \mathrm{C}_{14} \mathrm{H}_{10} ;[120-12-7] \\
\text { (2) Heptane; } \mathrm{C}_{7} \mathrm{H}_{16} ;[142-82-5] \\
\text { (3) 2-Butanol; } \mathrm{C}_{4} \mathrm{H}_{10} \mathrm{O} ;[78-92-2] \\
\text { (4) 1-Pentanol; } \mathrm{C}_{5} \mathrm{H}_{12} \mathrm{O} ;[71-41-0]\end{array}$ & $\begin{array}{l}\text { Original Measurements: } \\
{ }^{8} \text { A. Proctor, B. A. Martine, and } \\
\text { W. E. Acree, Jr., J. Chem. Eng. } \\
\text { Data 53, } 2197 \text { (2008). }\end{array}$ \\
\hline $\begin{array}{l}\text { Variables: } \\
T / \mathrm{K}=298.15 ; \text { Solvent Composition }\end{array}$ & $\begin{array}{l}\text { Prepared by: } \\
\text { W. E. Acree, Jr. }\end{array}$ \\
\hline
\end{tabular}


Experimental Values

Solubility of anthracene in heptane +2 -butanol +1 -pentanol mixtures ${ }^{\mathrm{a}}$

\begin{tabular}{lcccc}
\hline \hline$x_{2}{ }^{(\mathrm{s})}$ & $x_{3}{ }^{(\mathrm{s})}$ & $x_{2}$ & $x_{3}$ & $x_{1}$ \\
\hline 0.0000 & 0.0000 & 0.0000 & 0.0000 & 0.001097 \\
1.0000 & 0.0000 & 0.9984 & 0.0000 & 0.001571 \\
0.0000 & 1.0000 & 0.0000 & 0.9994 & 0.000585 \\
0.0686 & 0.7690 & 0.0685 & 0.7684 & 0.000780 \\
0.0800 & 0.2279 & 0.0799 & 0.2277 & 0.001085 \\
0.0882 & 0.5597 & 0.0881 & 0.5592 & 0.000911 \\
0.0886 & 0.4578 & 0.0885 & 0.4574 & 0.000952 \\
0.1131 & 0.7609 & 0.1130 & 0.7603 & 0.000834 \\
0.1263 & 0.1641 & 0.1262 & 0.1639 & 0.001153 \\
0.1368 & 0.7832 & 0.1367 & 0.7825 & 0.000843 \\
0.1814 & 0.1294 & 0.1812 & 0.1292 & 0.001232 \\
0.1875 & 0.5670 & 0.1873 & 0.5664 & 0.001002 \\
0.2017 & 0.3049 & 0.2015 & 0.3045 & 0.001152 \\
0.2475 & 0.4092 & 0.2472 & 0.4087 & 0.001147 \\
0.2984 & 0.5998 & 0.2981 & 0.5991 & 0.001117 \\
0.3415 & 0.1255 & 0.3410 & 0.1253 & 0.001376 \\
0.3945 & 0.3312 & 0.3940 & 0.3308 & 0.001319 \\
0.4013 & 0.4860 & 0.4008 & 0.4854 & 0.001259 \\
0.4193 & 0.1307 & 0.4187 & 0.1305 & 0.001445 \\
0.5817 & 0.2774 & 0.5809 & 0.2770 & 0.001471 \\
0.6022 & 0.1372 & 0.6013 & 0.1370 & 0.001556 \\
0.6251 & 0.2052 & 0.6242 & 0.2049 & 0.001514 \\
\hline$x_{2}(\mathrm{~s})$ & & 0.259
\end{tabular}

${ }^{\mathrm{a}} x_{2}{ }^{(\mathrm{s})}$ : initial mole fraction of component 2 in the ternary solvent mixture, $x_{3}{ }^{(\mathrm{s})}$ : initial mole fraction of component 3 in the ternary solvent mixture; $x_{1}$ : mole fraction solubility of the solute; $x_{2}$ : mole fraction of component 2 in the quaternary solution; $x_{3}$ : mole fraction of component 3 in the quaternary solution.

\section{Auxiliary Information}

Method/Apparatus/Procedure:

Constant-temperature bath, calorimetric thermometer, and an ultraviolet/ visible spectrophotometer.

Ternary solvent mixtures were prepared by mass. Excess solute and solvent were placed in amber glass bottles and allowed to equilibrate for several days at constant temperature. Attainment of equilibrium was verified by several repetitive measurements and by approaching equilibrium from supersaturation. Aliquots of saturated solutions were transferred through a coarse filter into volumetric flasks, weighed and diluted with methanol. Molar concentrations were determined by spectrophotometric measurements at $356 \mathrm{~nm}$.

Source and Purity of Chemicals:

(1) $99+\%$, Aldrich Chemical Company, Milwaukee, WI, USA, was recrystallized several times from acetone.

(2) $99 \%$, anhydrous, Aldrich Chemical Company, Milwaukee, WI, USA.

(3) $99+\%$, anhydrous, Aldrich Chemical Company.

(4) $99 \%$, Aldrich Chemical Company.

Components 2-4 were stored over molecular sieves and distilled shortly before use.

\section{Estimated Error:}

Temperature: $\pm 0.05 \mathrm{~K}$.

$x_{2}^{(\mathrm{s})}: \pm 0.0001$

$x_{3}{ }^{(\mathrm{s})}: \pm 0.0001$.

$x_{1}: \pm 1.5 \%$ (relative error).

\subsection{Anthracene solubility data in ternary cyclohexane + alcohol + alcohol solvent mixtures}

\begin{tabular}{ll}
\hline \hline Components: & $\begin{array}{l}\text { Original Measurements: } \\
\text { (1) Anthracene; } \mathrm{C}_{14} \mathrm{H}_{10} ;[120-12-7]\end{array}$ \\
$\begin{array}{l}\text { 28 T. Deng and W. E. Acree, Jr., J. } \\
\text { (2) 1-Propanol; } \mathrm{C}_{3} \mathrm{H}_{8} \mathrm{O} ;[71-23-8]\end{array}$ & Chem. Eng. Data 43, 1062 (1998). \\
(3) 1-Butanol; $\mathrm{C}_{4} \mathrm{H}_{10} \mathrm{O} ;[71-36-3]$ & \\
(4) Cyclohexane; $\mathrm{C}_{6} \mathrm{H}_{12} ;[110-82-7]$ & \\
\hline Variables: & Prepared by: \\
$T / \mathrm{K}=298.15 ;$ Solvent Composition & W. E. Acree, Jr. \\
\hline
\end{tabular}

Experimental Values

Solubility of anthracene in 1-propanol +1 -butanol + cyclohexane mixtures $^{\mathrm{a}}$

\begin{tabular}{lcccl}
\hline \hline$x_{2}^{(\mathrm{s})}$ & $x_{3}{ }^{(\mathrm{s})}$ & $x_{2}$ & \multicolumn{1}{c}{$x_{3}$} & \multicolumn{1}{c}{$x_{1}$} \\
\hline 0.0000 & 0.0000 & 0.0000 & 0.0000 & 0.001553 \\
1.0000 & 0.0000 & 0.9994 & 0.0000 & 0.000591 \\
0.0000 & 1.0000 & 0.0000 & 0.9992 & 0.000801 \\
0.4028 & 0.3242 & 0.4024 & 0.3239 & 0.00103 \\
0.1855 & 0.6881 & 0.1853 & 0.6875 & 0.000908 \\
0.3161 & 0.2543 & 0.3157 & 0.2540 & 0.00124 \\
0.3021 & 0.4885 & 0.3018 & 0.4880 & 0.000962 \\
0.7549 & 0.0932 & 0.7543 & 0.0931 & 0.000801 \\
0.7477 & 0.1763 & 0.7472 & 0.1762 & 0.000722 \\
0.2323 & 0.6835 & 0.2321 & 0.6829 & 0.000845 \\
0.1248 & 0.5214 & 0.1247 & 0.5208 & 0.00117 \\
0.4545 & 0.4645 & 0.4541 & 0.4641 & 0.000792 \\
0.5566 & 0.3649 & 0.5562 & 0.3646 & 0.000758 \\
0.2050 & 0.1611 & 0.2047 & 0.1608 & 0.00147 \\
0.7561 & 0.1317 & 0.7555 & 0.1316 & 0.000762 \\
0.5720 & 0.2322 & 0.5715 & 0.2320 & 0.000898 \\
0.1425 & 0.2171 & 0.1423 & 0.2168 & 0.00146 \\
0.1260 & 0.7052 & 0.1259 & 0.7045 & 0.000961 \\
0.2620 & 0.1061 & 0.2616 & 0.1059 & 0.00145 \\
0.1323 & 0.4225 & 0.1321 & 0.4220 & 0.00128 \\
0.4857 & 0.1009 & 0.4851 & 0.1008 & 0.00116 \\
0.5820 & 0.0966 & 0.5814 & 0.0965 & 0.00104 \\
\hline$x_{2}(\mathrm{~s}: 1210$
\end{tabular}

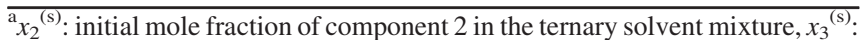
initial mole fraction of component 3 in the ternary solvent mixture; $x_{1}$ : mole fraction solubility of the solute; $x_{2}$ : mole fraction of component 2 in the quaternary solution; $x_{3}$ : mole fraction of component 3 in the quaternary solution.

\section{Auxiliary Information}

\section{Method/Apparatus/Procedure:}

Constant-temperature bath, calorimetric thermometer, and an ultraviolet/ visible spectrophotometer.

Ternary solvent mixtures were prepared by mass. Excess solute and solvent were placed in amber glass bottles and allowed to equilibrate for several days at constant temperature. Attainment of equilibrium was verified by several repetitive measurements and by approaching equilibrium from supersaturation. Aliquots of saturated solutions were transferred through a coarse filter into volumetric flasks, weighed and diluted with methanol. Molar concentrations were determined by spectrophotometric measurements at $356 \mathrm{~nm}$. 
Source and Purity of Chemicals:

(1) $99+\%$, Acros Chemical Company, USA, recrystallized three times from acetone.

(2) $99+\%$, anhydrous, Aldrich Chemical Company, Milwaukee, WI, USA.

(3) $99.8 \%$, HPLC grade, Aldrich Chemical Company.

(4) $99.9+\%$, HPLC grade, Aldrich Chemical Company.

Components $2-4$ were stored over molecular sieves and distilled shortly before use.

Estimated Error:

Temperature: $\pm 0.1 \mathrm{~K}$

$x_{2}{ }^{(\mathrm{s})}: \pm 0.0001$.

$x_{3}{ }^{(\mathrm{s})}: \pm 0.0001$

$x_{1}: \pm 1.5 \%$ (relative error).

\section{Components:}

(1) Anthracene; $\mathrm{C}_{14} \mathrm{H}_{10} ;[120-12-7]$

(2) 1-Propanol; $\mathrm{C}_{3} \mathrm{H}_{8} \mathrm{O}$; [71-23-8]

(3) 2-Butanol; $\mathrm{C}_{4} \mathrm{H}_{10} \mathrm{O}$; [78-92-2]

(4) Cyclohexane; $\mathrm{C}_{6} \mathrm{H}_{12} ;[110-82-7]$

\begin{tabular}{ll}
\hline Variables: & Prepared by: \\
$T / \mathrm{K}=298.15 ;$ Solvent Composition & W. E. Acree, Jr. \\
\hline
\end{tabular}

\section{Experimental Values}

Solubility of anthracene in 1-propanol + 2-butanol + cyclohexane mixtures ${ }^{\mathrm{a}}$

\begin{tabular}{lcccl}
\hline \hline$x_{2}{ }^{(\mathrm{s})}$ & $x_{3}{ }^{(\mathrm{s})}$ & $x_{2}$ & $x_{3}$ & \multicolumn{1}{c}{$x_{1}$} \\
\hline 0.0000 & 0.0000 & 0.0000 & 0.0000 & 0.001553 \\
1.0000 & 0.0000 & 0.9994 & 0.0000 & 0.000591 \\
0.0000 & 1.0000 & 0.0000 & 0.9994 & 0.000585 \\
0.3954 & 0.3274 & 0.3950 & 0.3271 & 0.000968 \\
0.1829 & 0.6898 & 0.1828 & 0.6893 & 0.000744 \\
0.3100 & 0.2560 & 0.3096 & 0.2557 & 0.00118 \\
0.2990 & 0.4892 & 0.2987 & 0.4888 & 0.000865 \\
0.7618 & 0.0907 & 0.7612 & 0.0906 & 0.000783 \\
0.7512 & 0.1751 & 0.7507 & 0.1750 & 0.000676 \\
0.2376 & 0.6800 & 0.2374 & 0.6795 & 0.000687 \\
0.1330 & 0.5172 & 0.1329 & 0.5167 & 0.00106 \\
0.4588 & 0.4628 & 0.4585 & 0.4625 & 0.000672 \\
0.5606 & 0.3622 & 0.5602 & 0.3620 & 0.000676 \\
0.1987 & 0.1628 & 0.1984 & 0.1626 & 0.00144 \\
0.7537 & 0.1327 & 0.7532 & 0.1326 & 0.000722 \\
0.5693 & 0.2327 & 0.5688 & 0.2325 & 0.000842 \\
0.1343 & 0.2183 & 0.1341 & 0.2180 & 0.00147 \\
0.1273 & 0.7061 & 0.1272 & 0.7055 & 0.000807 \\
0.2663 & 0.1078 & 0.2659 & 0.1076 & 0.00143 \\
0.1323 & 0.4217 & 0.1321 & 0.4212 & 0.00122 \\
0.4864 & 0.0989 & 0.4858 & 0.0988 & 0.00115 \\
0.5819 & 0.0959 & 0.5813 & 0.0958 & 0.00101 \\
\hline$x_{2}(\mathrm{~s}: 17195$
\end{tabular}

${ }^{\mathrm{a}} x_{2}{ }^{(\mathrm{s})}$ : initial mole fraction of component 2 in the ternary solvent mixture, $x_{3}{ }^{(\mathrm{s})}$ : initial mole fraction of component 3 in the ternary solvent mixture; $x_{1}$ : mole fraction solubility of the solute; $x_{2}$ : mole fraction of component 2 in the quaternary solution; $x_{3}$ : mole fraction of component 3 in the quaternary solution.

\section{Auxiliary Information}

\section{Method/Apparatus/Procedure:}

Constant-temperature bath, calorimetric thermometer, and an ultraviolet/ visible spectrophotometer.
Ternary solvent mixtures were prepared by mass. Excess solute and solvent were placed in amber glass bottles and allowed to equilibrate for several days at constant temperature. Attainment of equilibrium was verified by several repetitive measurements and by approaching equilibrium from supersaturation. Aliquots of saturated solutions were transferred through a coarse filter into volumetric flasks, weighed and diluted with methanol. Molar concentrations were determined by spectrophotometric measurements at $356 \mathrm{~nm}$.

\section{Source and Purity of Chemicals:}

(1) $99+\%$, Acros Chemical Company, USA, recrystallized three times from acetone.

(2) $99+\%$, anhydrous, Aldrich Chemical Company, Milwaukee, WI, USA. (3) $99+\%$, anhydrous, Aldrich Chemical Company.

(4) $99.9+\%$, HPLC grade, Aldrich Chemical Company.

Components $2-4$ were stored over molecular sieves and distilled shortly before use.

\section{Estimated Error:}

Temperature: $\pm 0.1 \mathrm{~K}$.

$x_{2}{ }^{(s)}: \pm 0.0001$.

$x_{3}{ }^{(\mathrm{s})}: \pm 0.0001$.

$x_{1}: \pm 1.5 \%$ (relative error).

\begin{tabular}{ll}
\hline \hline Components: & $\begin{array}{l}\text { Original Measurements: } \\
\text { (1) Anthracene; } \mathrm{C}_{14} \mathrm{H}_{10} ;[120-12-7]\end{array}$ \\
$\begin{array}{l}{ }^{28} \mathrm{~T} \text {. Deng and W. E. Acree, Jr., J. } \\
\text { (2) 2-Propanol; } \mathrm{C}_{3} \mathrm{H}_{8} \mathrm{O} ;[67-63-0]\end{array}$ & Chem. Eng. Data 43, 1062 (1998). \\
(3) 1-Butanol; $\mathrm{C}_{4} \mathrm{H}_{10} \mathrm{O} ;[71-36-3]$ & \\
(4) Cyclohexane; $\mathrm{C}_{6} \mathrm{H}_{12} ;[110-82-7]$ & \\
\hline Variables: & Prepared by: \\
$T / \mathrm{K}=298.15 ;$ Solvent Composition & W. E. Acree, Jr. \\
\hline
\end{tabular}

Experimental Values

Solubility of anthracene in 2-propanol +1 -butanol + cyclohexane mixtures $^{\mathrm{a}}$

\begin{tabular}{lcccl}
\hline \hline$x_{2}{ }^{(\mathrm{s})}$ & $x_{3}{ }^{(\mathrm{s})}$ & $x_{2}$ & \multicolumn{1}{c}{$x_{3}$} & \multicolumn{1}{c}{$x_{1}$} \\
\hline 0.0000 & 0.0000 & 0.0000 & 0.0000 & 0.001553 \\
1.0000 & 0.0000 & 0.9996 & 0.0000 & 0.000411 \\
0.0000 & 1.0000 & 0.0000 & 0.9992 & 0.000801 \\
0.3954 & 0.3278 & 0.3950 & 0.3275 & 0.000947 \\
0.1837 & 0.6868 & 0.1835 & 0.6862 & 0.000865 \\
0.2950 & 0.2483 & 0.2946 & 0.2480 & 0.00122 \\
0.3002 & 0.4961 & 0.2999 & 0.4957 & 0.000894 \\
0.7621 & 0.0887 & 0.7616 & 0.0886 & 0.000657 \\
0.7514 & 0.1725 & 0.7510 & 0.1724 & 0.000583 \\
0.2348 & 0.6791 & 0.2346 & 0.6786 & 0.000798 \\
0.1296 & 0.5185 & 0.1295 & 0.5179 & 0.00113 \\
0.4555 & 0.4697 & 0.4552 & 0.4694 & 0.000700 \\
0.5520 & 0.3711 & 0.5516 & 0.3709 & 0.000662 \\
0.2010 & 0.1705 & 0.2007 & 0.1703 & 0.00143 \\
0.7444 & 0.1414 & 0.7437 & 0.1413 & 0.000629 \\
0.5644 & 0.2340 & 0.5639 & 0.2338 & 0.000799 \\
0.1469 & 0.2222 & 0.1467 & 0.2219 & 0.00144 \\
0.1256 & 0.7022 & 0.1255 & 0.7015 & 0.000929 \\
0.2530 & 0.1066 & 0.2526 & 0.1064 & 0.00141 \\
0.1564 & 0.4067 & 0.1562 & 0.4062 & 0.00123 \\
0.4811 & 0.1002 & 0.4806 & 0.1001 & 0.00108 \\
0.5771 & 0.1016 & 0.5766 & 0.1015 & 0.000936 \\
\hline
\end{tabular}

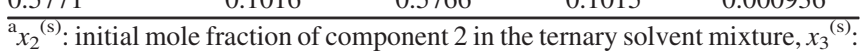
initial mole fraction of component 3 in the ternary solvent mixture; $x_{1}$ : mole fraction solubility of the solute; $x_{2}$ : mole fraction of component 2 in the quaternary solution; $x_{3}$ : mole fraction of component 3 in the quaternary solution. 


\section{Auxiliary Information}

\section{Method/Apparatus/Procedure:}

Constant-temperature bath, calorimetric thermometer, and an ultraviolet/ visible spectrophotometer.

Ternary solvent mixtures were prepared by mass. Excess solute and solvent were placed in amber glass bottles and allowed to equilibrate for several days at constant temperature. Attainment of equilibrium was verified by several repetitive measurements and by approaching equilibrium from supersaturation. Aliquots of saturated solutions were transferred through a coarse filter into volumetric flasks, weighed and diluted with methanol. Molar concentrations were determined by spectrophotometric measurements at $356 \mathrm{~nm}$.

\section{Source and Purity of Chemicals:}

(1) $99+\%$, Acros Chemical Company, USA, recrystallized three times from acetone.

(2) $99+\%$, anhydrous, Aldrich Chemical Company, Milwaukee, WI, USA.

(3) $99.8 \%$, HPLC grade, Aldrich Chemical Company.

(4) $99.9+\%$, HPLC grade, Aldrich Chemical Company.

Components $2-4$ were stored over molecular sieves and distilled shortly before use.

\section{Estimated Error:}

Temperature: $\pm 0.1 \mathrm{~K}$

$x_{2}{ }^{(\mathrm{s})}: \pm 0.0001$.

$x_{3}{ }^{(\mathrm{s})}: \pm 0.0001$

$x_{1}: \pm 1.5 \%$ (relative error)

\section{Components:}

(1) Anthracene; $\mathrm{C}_{14} \mathrm{H}_{10} ;[120-12-7]$

(2) 2-Propanol; $\mathrm{C}_{3} \mathrm{H}_{8} \mathrm{O}$; [67-63-0]

(3) 2-Butanol; $\mathrm{C}_{4} \mathrm{H}_{10} \mathrm{O}$; [78-92-2]

(4) Cyclohexane; $\mathrm{C}_{6} \mathrm{H}_{12} ;$ [110-82-7]

\begin{tabular}{ll}
\hline Variables: & Prepared by: \\
$T / \mathrm{K}=298.15 ;$ Solvent Composition & W. E. Acree, Jr. \\
\hline
\end{tabular}

\section{Experimental Values}

Solubility of anthracene in 2-propanol +2 -butanol + cyclohexane mixtures

\begin{tabular}{lcccl}
\hline \hline$x_{2}{ }^{(\mathrm{s})}$ & $x_{3}{ }^{(\mathrm{s})}$ & $x_{2}$ & $x_{3}$ & \multicolumn{1}{c}{$x_{1}$} \\
\hline 0.0000 & 0.0000 & 0.0000 & 0.0000 & 0.001553 \\
1.0000 & 0.0000 & 0.9996 & 0.0000 & 0.000411 \\
0.0000 & 1.0000 & 0.0000 & 0.9994 & 0.000585 \\
0.3961 & 0.3220 & 0.3957 & 0.3217 & 0.000901 \\
0.1687 & 0.7065 & 0.1686 & 0.7060 & 0.000718 \\
0.2997 & 0.2619 & 0.2994 & 0.2616 & 0.00115 \\
0.2991 & 0.4964 & 0.2989 & 0.4958 & 0.000797 \\
0.7598 & 0.0919 & 0.7593 & 0.0918 & 0.000641 \\
0.7422 & 0.1829 & 0.7418 & 0.1828 & 0.000550 \\
0.2324 & 0.6804 & 0.2322 & 0.6799 & 0.000651 \\
0.1274 & 0.5218 & 0.1273 & 0.5213 & 0.00104 \\
0.4458 & 0.4727 & 0.4455 & 0.4724 & 0.000599 \\
0.5459 & 0.3711 & 0.5456 & 0.3709 & 0.000600 \\
0.1891 & 0.1695 & 0.1888 & 0.1693 & 0.00141 \\
0.7587 & 0.1331 & 0.7583 & 0.1330 & 0.000591 \\
0.5631 & 0.2387 & 0.5627 & 0.2385 & 0.000738
\end{tabular}

$\begin{array}{lllll}0.1236 & 0.2196 & 0.1234 & 0.2193 & 0.00144 \\ 0.1233 & 0.7068 & 0.1232 & 0.7062 & 0.000789 \\ 0.2564 & 0.1142 & 0.2560 & 0.1140 & 0.00138 \\ 0.1228 & 0.4273 & 0.1227 & 0.4268 & 0.00120 \\ 0.4746 & 0.1033 & 0.4741 & 0.1032 & 0.00106 \\ 0.5815 & 0.0972 & 0.5810 & 0.0971 & 0.000911\end{array}$

${ }^{\mathrm{a}} x_{2}{ }^{(\mathrm{s})}$ : initial mole fraction of component 2 in the ternary solvent mixture, $x_{3}{ }^{(\mathrm{s})}$ : initial mole fraction of component 3 in the ternary solvent mixture; $x_{1}$ : mole fraction solubility of the solute; $x_{2}$ : mole fraction of component 2 in the quaternary solution; $x_{3}$ : mole fraction of component 3 in the quaternary solution.

\section{Method/Apparatus/Procedure:}

Constant-temperature bath, calorimetric thermometer, and an ultraviolet/ visible spectrophotometer.

Ternary solvent mixtures were prepared by mass. Excess solute and solvent were placed in amber glass bottles and allowed to equilibrate for several days at constant temperature. Attainment of equilibrium was verified by several repetitive measurements and by approaching equilibrium from supersaturation. Aliquots of saturated solutions were transferred through a coarse filter into volumetric flasks, weighed and diluted with methanol. Molar concentrations were determined by spectrophotometric measurements at $356 \mathrm{~nm}$.

\section{Source and Purity of Chemicals:}

(1) $99+\%$, Acros Chemical Company, USA, recrystallized three times from acetone.

(2) $99+\%$, anhydrous, Aldrich Chemical Company, Milwaukee, WI, USA.

(3) $99+\%$, anhydrous, Aldrich Chemical Company.

(4) $99.9+\%$, HPLC grade, Aldrich Chemical Company.

Components 2-4 were stored over molecular sieves and distilled shortly before use.

\section{Estimated Error:}

Temperature: $\pm 0.1 \mathrm{~K}$.

$x_{2}{ }^{(s)}: \pm 0.0001$.

$x_{3}{ }^{(\mathrm{s})}: \pm 0.0001$.

$x_{1}: \pm 1.5 \%$ (relative error).

\begin{tabular}{|c|c|}
\hline $\begin{array}{l}\text { Components: } \\
\text { (1) Anthracene; } \mathrm{C}_{14} \mathrm{H}_{10} ;[120-12-7] \\
\text { (2) Cyclohexane; } \mathrm{C}_{6} \mathrm{H}_{12} ;[110-82-7] \\
\text { (3) 1-Propanol; } \mathrm{C}_{3} \mathrm{H}_{8} \mathrm{O} ;[71-23-8] \\
\text { (4) 2-Methyl-1-propanol; } \mathrm{C}_{4} \mathrm{H}_{10} \mathrm{O} \text {; } \\
\text { [78-83-1] }\end{array}$ & $\begin{array}{l}\text { Original Measurements: } \\
{ }^{7} \text { A. Proctor, B. H. Blake-Taylor, } \\
\text { and W. E. Acree, Jr., J. Chem. } \\
\text { Eng. Data 53, } 2910 \text { (2008). }\end{array}$ \\
\hline $\begin{array}{l}\text { Variables: } \\
T / \mathrm{K}=298.15 ; \text { Solvent Composition }\end{array}$ & $\begin{array}{l}\text { Prepared by: } \\
\text { W. E. Acree, Jr. }\end{array}$ \\
\hline
\end{tabular}


Experimental Values

Solubility of anthracene in cyclohexane +1 -propanol +2 -methyl-1-propanol mixtures $^{\mathrm{a}}$

\begin{tabular}{lcccc}
\hline \hline$x_{2}{ }^{(\mathrm{s})}$ & $x_{3}{ }^{(\mathrm{s})}$ & $x_{2}$ & $x_{3}$ & $x_{1}$ \\
\hline 0.0000 & 0.0000 & 0.0000 & 0.0000 & 0.000470 \\
1.0000 & 0.0000 & 0.9984 & 0.0000 & 0.001554 \\
0.0000 & 1.0000 & 0.0000 & 0.9994 & 0.000591 \\
0.0668 & 0.7420 & 0.0668 & 0.7415 & 0.000671 \\
0.0865 & 0.2351 & 0.0864 & 0.2350 & 0.000625 \\
0.0896 & 0.5500 & 0.0895 & 0.5496 & 0.000676 \\
0.0901 & 0.4509 & 0.0900 & 0.4506 & 0.000647 \\
0.1161 & 0.7317 & 0.1160 & 0.7312 & 0.000743 \\
0.1422 & 0.1786 & 0.1421 & 0.1785 & 0.000688 \\
0.1589 & 0.7473 & 0.1588 & 0.7467 & 0.000811 \\
0.1811 & 0.1157 & 0.1810 & 0.1156 & 0.000727 \\
0.2057 & 0.5728 & 0.2055 & 0.5723 & 0.000847 \\
0.2238 & 0.2954 & 0.2236 & 0.2952 & 0.000816 \\
0.2716 & 0.3631 & 0.2714 & 0.3628 & 0.000911 \\
0.3200 & 0.5780 & 0.3197 & 0.5774 & 0.001030 \\
0.3478 & 0.1382 & 0.3475 & 0.1381 & 0.000955 \\
0.4064 & 0.4722 & 0.4059 & 0.4717 & 0.001147 \\
0.4324 & 0.1479 & 0.4319 & 0.1477 & 0.001095 \\
0.4404 & 0.3073 & 0.4399 & 0.3069 & 0.001157 \\
0.6135 & 0.1632 & 0.6126 & 0.1630 & 0.001393 \\
0.6309 & 0.2730 & 0.6300 & 0.2726 & 0.001443 \\
0.6356 & 0.1853 & 0.6347 & 0.1850 & 0.001432 \\
\hline
\end{tabular}

${ }^{\mathrm{a}} x_{2}{ }^{(\mathrm{s})}$ : initial mole fraction of component 2 in the ternary solvent mixture, $x_{3}{ }^{(\mathrm{s})}$ : initial mole fraction of component 3 in the ternary solvent mixture; $x_{1}$ : mole fraction solubility of the solute; $x_{2}$ : mole fraction of component 2 in the quaternary solution; $x_{3}$ : mole fraction of component 3 in the quaternary solution.

\section{Auxiliary Information}

\section{Method/Apparatus/Procedure:}

Constant-temperature bath, calorimetric thermometer, and an ultraviolet/ visible spectrophotometer.

Ternary solvent mixtures were prepared by mass. Excess solute and solvent were placed in amber glass bottles and allowed to equilibrate for several days at constant temperature. Attainment of equilibrium was verified by several repetitive measurements and by approaching equilibrium from supersaturation. Aliquots of saturated solutions were transferred through a coarse filter into volumetric flasks, weighed and diluted with methanol. Molar concentrations were determined by spectrophotometric measurements at $356 \mathrm{~nm}$.

\section{Source and Purity of Chemicals:}

(1) $99+\%$, Acros Chemical Company, USA, recrystallized several times from acetone.

(2) $99.5 \%$, anhydrous, Aldrich Chemical Company, Milwaukee, WI, USA.

(3) $99.7 \%$, anhydrous, Aldrich Chemical Company.

(4) $99.5 \%$, anhydrous, Aldrich Chemical Company.

Components $2-4$ were stored over molecular sieves and distilled shortly before use.

\section{Estimated Error:}

Temperature: $\pm 0.05 \mathrm{~K}$.

$x_{2}^{(\mathrm{s})}: \pm 0.0001$.

$x_{3}{ }^{(\mathrm{s})}: \pm 0.0001$.

$x_{1}: \pm 1.5 \%$ (relative error).
Components:

(1) Anthracene; $\mathrm{C}_{14} \mathrm{H}_{10} ;[120-12-7]$

(2) Cyclohexane; $\mathrm{C}_{6} \mathrm{H}_{12} ;[110-82-7]$

(3) 2-Propanol; $\mathrm{C}_{3} \mathrm{H}_{8} \mathrm{O}$; [67-63-0]

(4) 2-Methyl-1-propanol; $\mathrm{C}_{4} \mathrm{H}_{10} \mathrm{O}$; [78-83-1]

\begin{tabular}{ll}
\hline Variables: & Prepared by: \\
$T / \mathrm{K}=298.15$; Solvent Composition & W. E. Acree, Jr. \\
\hline
\end{tabular}

Experimental Values

Solubility of anthracene in cyclohexane +2 -propanol +2 -methyl-1-propanol mixtures $^{\mathrm{a}}$

\begin{tabular}{lcccc}
\hline \hline$x_{2}^{(\mathrm{s})}$ & $x_{3}{ }^{(\mathrm{s})}$ & $x_{2}$ & $x_{3}$ & $x_{1}$ \\
\hline 0.0000 & 0.0000 & 0.0000 & 0.0000 & 0.000470 \\
1.0000 & 0.0000 & 0.9984 & 0.0000 & 0.001554 \\
0.0000 & 1.0000 & 0.0000 & 0.9996 & 0.000411 \\
0.0695 & 0.7549 & 0.0695 & 0.7545 & 0.000532 \\
0.0808 & 0.2249 & 0.0808 & 0.2248 & 0.000569 \\
0.0858 & 0.4635 & 0.0858 & 0.4632 & 0.000563 \\
0.0875 & 0.5532 & 0.0875 & 0.5529 & 0.000565 \\
0.1220 & 0.1973 & 0.1219 & 0.1972 & 0.000619 \\
0.1233 & 0.7353 & 0.1232 & 0.7348 & 0.000604 \\
0.1585 & 0.7448 & 0.1583 & 0.7441 & 0.000967 \\
0.1863 & 0.1573 & 0.1862 & 0.1572 & 0.000711 \\
0.2040 & 0.3139 & 0.2038 & 0.3137 & 0.000737 \\
0.2067 & 0.5534 & 0.2065 & 0.5530 & 0.000739 \\
0.2783 & 0.3966 & 0.2781 & 0.3963 & 0.000840 \\
0.3208 & 0.5835 & 0.3205 & 0.5830 & 0.000909 \\
0.3505 & 0.1361 & 0.3502 & 0.1360 & 0.000921 \\
0.4201 & 0.4833 & 0.4196 & 0.4828 & 0.001081 \\
0.4224 & 0.3102 & 0.4220 & 0.3099 & 0.001061 \\
0.4482 & 0.1322 & 0.4477 & 0.1321 & 0.001084 \\
0.6321 & 0.1976 & 0.6312 & 0.1973 & 0.001373 \\
0.6322 & 0.2695 & 0.6313 & 0.2691 & 0.001383 \\
0.6482 & 0.1305 & 0.6473 & 0.1303 & 0.001383 \\
\hline
\end{tabular}

${ }^{\mathrm{a}} x_{2}{ }^{(\mathrm{s})}$ : initial mole fraction of component 2 in the ternary solvent mixture, $x_{3}{ }^{(\mathrm{s})}$ : initial mole fraction of component 3 in the ternary solvent mixture; $x_{1}$ : mole fraction solubility of the solute; $x_{2}$ : mole fraction of component 2 in the quaternary solution; $x_{3}$ : mole fraction of component 3 in the quaternary solution.

\section{Auxiliary Information}

\section{Method/Apparatus/Procedure:}

Constant-temperature bath, calorimetric thermometer, and an ultraviolet/ visible spectrophotometer.

Ternary solvent mixtures were prepared by mass. Excess solute and solvent were placed in amber glass bottles and allowed to equilibrate for several days at constant temperature. Attainment of equilibrium was verified by several repetitive measurements and by approaching equilibrium from supersaturation. Aliquots of saturated solutions were transferred through a coarse filter into volumetric flasks, weighed and diluted with methanol. Molar concentrations were determined by spectrophotometric measurements at $356 \mathrm{~nm}$.

Source and Purity of Chemicals:

(1) $99+\%$, Acros Chemical Company, USA, recrystallized several times from acetone.

(2) $99.5 \%$, anhydrous, Aldrich Chemical Company, Milwaukee, WI, USA.

(3) $99.5 \%$, anhydrous, Aldrich Chemical Company.

(4) $99.5 \%$, anhydrous, Aldrich Chemical Company.

Components 2-4 were stored over molecular sieves and distilled shortly before use. 
Estimated Error:

Temperature: $\pm 0.05 \mathrm{~K}$.

$x_{2}{ }^{(\mathrm{s})}: \pm 0.0001$

$x_{3}{ }^{(\mathrm{s})}: \pm 0.0001$.

$x_{1}: \pm 1.5 \%$ (relative error).

\begin{tabular}{|c|c|}
\hline $\begin{array}{l}\text { Components: } \\
\text { (1) Anthracene; } \mathrm{C}_{14} \mathrm{H}_{10} ;[120-12-7] \\
\text { (2) Cyclohexane; } \mathrm{C}_{6} \mathrm{H}_{12} ;[110-82-7] \\
\text { (3) 1-Butanol; } \mathrm{C}_{4} \mathrm{H}_{10} \mathrm{O} ;[71-36-3] \\
\text { (4) 2-Methyl-1-propanol; } \mathrm{C}_{4} \mathrm{H}_{10} \mathrm{O} \text {; } \\
\text { [78-83-1] }\end{array}$ & $\begin{array}{l}\text { Original Measurements: } \\
{ }^{7} \text { A. Proctor, B. H. Blake-Taylor, } \\
\text { and W. E. Acree, Jr., J. Chem. } \\
\text { Eng. Data 53, } 2910 \text { (2008). }\end{array}$ \\
\hline $\begin{array}{l}\text { Variables: } \\
T / \mathrm{K}=298.15 \text {; Solvent Composition }\end{array}$ & $\begin{array}{l}\text { Prepared by: } \\
\text { W. E. Acree, Jr. }\end{array}$ \\
\hline
\end{tabular}

\section{Experimental Values}

Solubility of anthracene in cyclohexane +1 -butanol +2 -methyl-1-propanol mixtures $^{\mathrm{a}}$

\begin{tabular}{lcccc}
\hline \hline$x_{2}{ }^{(\mathrm{s})}$ & $x_{3}{ }^{(\mathrm{s})}$ & $x_{2}$ & $x_{3}$ & $x_{1}$ \\
\hline 0.0000 & 0.0000 & 0.0000 & 0.0000 & 0.000470 \\
1.0000 & 0.0000 & 0.9984 & 0.0000 & 0.001554 \\
0.0000 & 1.0000 & 0.0000 & 0.9992 & 0.000801 \\
0.0792 & 0.2125 & 0.0791 & 0.2124 & 0.000641 \\
0.0946 & 0.6945 & 0.0945 & 0.6939 & 0.000826 \\
0.1051 & 0.4014 & 0.1050 & 0.4011 & 0.000742 \\
0.1104 & 0.5029 & 0.1102 & 0.5024 & 0.000772 \\
0.1160 & 0.7209 & 0.1159 & 0.7203 & 0.000879 \\
0.1205 & 0.1660 & 0.1205 & 0.1659 & 0.000672 \\
0.1890 & 0.7009 & 0.1888 & 0.7002 & 0.000984 \\
0.1958 & 0.1042 & 0.1957 & 0.1041 & 0.000734 \\
0.2197 & 0.5280 & 0.2195 & 0.5275 & 0.000949 \\
0.2342 & 0.2856 & 0.2340 & 0.2854 & 0.000866 \\
0.2830 & 0.3497 & 0.2827 & 0.3494 & 0.000978 \\
0.3593 & 0.1287 & 0.3589 & 0.1286 & 0.000977 \\
0.3616 & 0.5304 & 0.3612 & 0.5298 & 0.001174 \\
0.4372 & 0.2703 & 0.4367 & 0.2700 & 0.001173 \\
0.4563 & 0.1092 & 0.4558 & 0.1091 & 0.001131 \\
0.4629 & 0.4308 & 0.4623 & 0.4302 & 0.001302 \\
0.6529 & 0.2289 & 0.6519 & 0.2285 & 0.001499 \\
0.6583 & 0.1182 & 0.6574 & 0.1180 & 0.001438 \\
0.6754 & 0.1627 & 0.6744 & 0.1625 & 0.001476 \\
\hline${ }^{a}{ }_{2}{ }^{(s)}:$ initial mole fraction of component 2 in the ternary solvent mixture, $x_{3}{ }^{(s)}$ : \\
initial mole fraction of component 3 in the ternary solvent mixture; $x_{1}:$ mole \\
fraction solubility of the solute; $x_{2}:$ mole fraction of component 2 in the \\
quaternary solution; $x_{3}:$ mole fraction of component 3 in the quaternary \\
solution. & & & & \\
& & & &
\end{tabular}

\section{Auxiliary Information}

\section{Method/Apparatus/Procedure:}

Constant-temperature bath, calorimetric thermometer, and an ultraviolet/ visible spectrophotometer.

Ternary solvent mixtures were prepared by mass. Excess solute and solvent were placed in amber glass bottles and allowed to equilibrate for several days at constant temperature. Attainment of equilibrium was verified by several repetitive measurements and by approaching equilibrium from supersaturation. Aliquots of saturated solutions were transferred through a coarse filter into volumetric flasks, weighed and diluted with methanol. Molar concentrations were determined by spectrophotometric measurements at $356 \mathrm{~nm}$.

Source and Purity of Chemicals:

(1) $99+\%$, Acros Chemical Company, USA, recrystallized several times from acetone.

(2) $99.5 \%$, anhydrous, Aldrich Chemical Company, Milwaukee, WI, USA.

(3) $99.8 \%$, anhydrous, Aldrich Chemical Company.

(4) $99.5 \%$, anhydrous, Aldrich Chemical Company.

Components $2-4$ were stored over molecular sieves and distilled shortly before use.

\section{Estimated Error:}

Temperature: $\pm 0.05 \mathrm{~K}$.

$x_{2}{ }^{(s)}: \pm 0.0001$.

$x_{3}{ }^{(\mathrm{s})}: \pm 0.0001$.

$x_{1}: \pm 1.5 \%$ (relative error).

\section{Components:}

(1) Anthracene; $\mathrm{C}_{14} \mathrm{H}_{10} ;$ [120-12-7]

(2) Cyclohexane; $\mathrm{C}_{6} \mathrm{H}_{12} ;[110-82-7]$

(3) 2-Butanol; $\mathrm{C}_{4} \mathrm{H}_{10} \mathrm{O}$; [78-92-2]

(4) 2-Methyl-1-propanol; $\mathrm{C}_{4} \mathrm{H}_{10} \mathrm{O}$;

[78-83-1]

\begin{tabular}{ll}
\hline Variables: & Prepared by: \\
$T / \mathrm{K}=298.15$; Solvent Composition & W. E. Acree, Jr. \\
\hline
\end{tabular}

Experimental Values

Solubility of anthracene in cyclohexane +2 -butanol +2 -methyl-1-propanol mixtures $^{\mathrm{a}}$

\begin{tabular}{lcccc}
\hline \hline$x_{2}^{(\mathrm{s})}$ & $x_{3}(\mathrm{~s})$ & $x_{2}$ & $x_{3}$ & $x_{1}$ \\
\hline 0.0000 & 0.0000 & 0.0000 & 0.0000 & 0.000470 \\
1.0000 & 0.0000 & 0.9984 & 0.0000 & 0.001554 \\
0.0000 & 1.0000 & 0.0000 & 0.9994 & 0.000585 \\
0.0892 & 0.4137 & 0.0891 & 0.4314 & 0.000637 \\
0.0920 & 0.2084 & 0.0919 & 0.2083 & 0.000610 \\
0.1010 & 0.6953 & 0.1009 & 0.6948 & 0.000704 \\
0.1037 & 0.5052 & 0.1036 & 0.5049 & 0.000675 \\
0.1307 & 0.1597 & 0.1306 & 0.1596 & 0.000649 \\
0.1387 & 0.7059 & 0.1386 & 0.7054 & 0.000755 \\
0.1843 & 0.1292 & 0.1842 & 0.1291 & 0.000718 \\
0.1898 & 0.6947 & 0.1896 & 0.6941 & 0.000841 \\
0.2162 & 0.2707 & 0.2160 & 0.2705 & 0.000775 \\
0.2306 & 0.5013 & 0.2304 & 0.5009 & 0.000859 \\
0.2961 & 0.3570 & 0.2958 & 0.3567 & 0.000916 \\
0.3551 & 0.1389 & 0.3548 & 0.1388 & 0.000968 \\
0.3613 & 0.5254 & 0.3609 & 0.5248 & 0.001080 \\
0.4508 & 0.2673 & 0.4503 & 0.2670 & 0.001162 \\
0.4596 & 0.1200 & 0.4591 & 0.1199 & 0.001176 \\
0.4843 & 0.3913 & 0.4837 & 0.3908 & 0.001236 \\
0.6427 & 0.1228 & 0.6418 & 0.1226 & 0.001412 \\
0.6438 & 0.1721 & 0.6429 & 0.1719 & 0.001416 \\
0.6704 & 0.2339 & 0.6694 & 0.2336 & 0.001479 \\
\hline
\end{tabular}

${ }^{\mathrm{a}} x_{2}{ }^{(\mathrm{s})}$ : initial mole fraction of component 2 in the ternary solvent mixture, $x_{3}{ }^{(\mathrm{s})}$ : initial mole fraction of component 3 in the ternary solvent mixture; $x_{1}$ : mole fraction solubility of the solute; $x_{2}$ : mole fraction of component 2 in the quaternary solution; $x_{3}$ : mole fraction of component 3 in the quaternary solution. 


\section{Auxiliary Information}

Method/Apparatus/Procedure:

Constant-temperature bath, calorimetric thermometer, and an ultraviolet/ visible spectrophotometer.

Ternary solvent mixtures were prepared by mass. Excess solute and solvent were placed in amber glass bottles and allowed to equilibrate for several days at constant temperature. Attainment of equilibrium was verified by several repetitive measurements and by approaching equilibrium from supersaturation. Aliquots of saturated solutions were transferred through a coarse filter into volumetric flasks, weighed and diluted with methanol. Molar concentrations were determined by spectrophotometric measurements at $356 \mathrm{~nm}$.

\section{Source and Purity of Chemicals:}

(1) $99+\%$, Acros Chemical Company, USA, recrystallized several times from acetone.

(2) $99.5 \%$, anhydrous, Aldrich Chemical Company, Milwaukee, WI, USA.

(3) $99.7 \%$, anhydrous, Aldrich Chemical Company.

(4) $99.5 \%$, anhydrous, Aldrich Chemical Company.

Components 2-4 were stored over molecular sieves and distilled shortly before use.

\section{Estimated Error:}

Temperature: $\pm 0.05 \mathrm{~K}$.

$x_{2}{ }^{(\mathrm{s})}: \pm 0.0001$.

$x_{3}{ }^{(\mathrm{s})}: \pm 0.0001$.

$x_{1}: \pm 1.5 \%$ (relative error).

\section{Components:}

(1) Anthracene; $\mathrm{C}_{14} \mathrm{H}_{10} ;[120-12-7]$

(2) Cyclohexane; $\mathrm{C}_{6} \mathrm{H}_{12} ;$ [110-82-7]

(3) 1-Propanol; $\mathrm{C}_{3} \mathrm{H}_{8} \mathrm{O}$; [71-23-8]

(4) 1-Pentanol; $\mathrm{C}_{5} \mathrm{H}_{12} \mathrm{O}$; [71-41-0]

\section{Original Measurements:}

${ }^{11}$ B. A. Martine, B. H. Brooke-

Taylor, and W. E. Acree, Jr., J.

Chem. Eng. Data 53, 556 (2008).

\begin{tabular}{ll}
\hline Variables: & Prepared by: \\
$T / \mathrm{K}=298.15 ;$ Solvent Composition & W. E. Acree, Jr. \\
\hline
\end{tabular}

Experimental Values

Solubility of anthracene in cyclohexane +1 -propanol +1 -pentanol mixtures ${ }^{\mathrm{a}}$

\begin{tabular}{lcccc}
\hline \hline$x_{2}{ }^{(\mathrm{s})}$ & $x_{3}{ }^{(\mathrm{s})}$ & $x_{2}$ & $x_{3}$ & $x_{1}$ \\
\hline 0.0000 & 0.0000 & 0.0000 & 0.0000 & 0.001097 \\
1.0000 & 0.0000 & 0.9984 & 0.0000 & 0.001553 \\
0.0000 & 1.0000 & 0.0000 & 0.9994 & 0.000591 \\
0.2935 & 0.4130 & 0.2932 & 0.4125 & 0.001169 \\
0.1625 & 0.1919 & 0.1623 & 0.1917 & 0.001157 \\
0.1155 & 0.7647 & 0.1154 & 0.7641 & 0.000828 \\
0.6479 & 0.2091 & 0.6469 & 0.2088 & 0.001524 \\
0.2247 & 0.3263 & 0.2244 & 0.3259 & 0.001132 \\
0.4451 & 0.3285 & 0.4445 & 0.3281 & 0.001327 \\
0.2065 & 0.5796 & 0.2063 & 0.5790 & 0.001008 \\
0.1001 & 0.2660 & 0.1000 & 0.2657 & 0.001068 \\
0.6766 & 0.1285 & 0.6755 & 0.1283 & 0.001597 \\
0.0917 & 0.7588 & 0.0916 & 0.7582 & 0.000810 \\
0.6331 & 0.2611 & 0.6322 & 0.2607 & 0.001484 \\
0.1875 & 0.1483 & 0.1873 & 0.1481 & 0.001197 \\
0.1561 & 0.7609 & 0.1560 & 0.7602 & 0.000863 \\
0.0988 & 0.4855 & 0.0987 & 0.4850 & 0.000960 \\
0.0859 & 0.5946 & 0.0858 & 0.5941 & 0.000896
\end{tabular}

$\begin{array}{lllll}0.3958 & 0.1298 & 0.3953 & 0.1296 & 0.001381 \\ 0.3232 & 0.5797 & 0.3228 & 0.5791 & 0.001114 \\ 0.4679 & 0.1395 & 0.4672 & 0.1393 & 0.001450 \\ 0.4296 & 0.4786 & 0.4291 & 0.4780 & 0.001251\end{array}$

${ }^{\mathrm{a}} x_{2}{ }^{(\mathrm{s})}$ : initial mole fraction of component 2 in the ternary solvent mixture, $x_{3}{ }^{(\mathrm{s})}$ : initial mole fraction of component 3 in the ternary solvent mixture; $x_{1}$ : mole fraction solubility of the solute; $x_{2}$ : mole fraction of component 2 in the quaternary solution; $x_{3}$ : mole fraction of component 3 in the quaternary solution.

\section{Auxiliary Information}

\section{Method/Apparatus/Procedure:}

Constant-temperature bath, calorimetric thermometer, and an ultraviolet/ visible spectrophotometer.

Ternary solvent mixtures were prepared by mass. Excess solute and solvent were placed in amber glass bottles and allowed to equilibrate for several days at constant temperature. Attainment of equilibrium was verified by several repetitive measurements and by approaching equilibrium from supersaturation. Aliquots of saturated solutions were transferred through a coarse filter into volumetric flasks, weighed and diluted with methanol. Molar concentrations were determined by spectrophotometric measurements at $356 \mathrm{~nm}$.

\section{Source and Purity of Chemicals:}

(1) $99+\%$, Aldrich Chemical Company, Milwaukee, WI, USA, was recrystallized several times from acetone.

(2) $99 \%$, anhydrous, Aldrich Chemical Company, Milwaukee, WI, USA.

(3) $99+\%$, anhydrous, Aldrich Chemical Company.

(4) $99 \%$, Aldrich Chemical Company.

Components 2-4 were stored over molecular sieves and distilled shortly before use.

\section{Estimated Error:}

Temperature: $\pm 0.05 \mathrm{~K}$.

$x_{2}{ }^{(\mathrm{s})}: \pm 0.0001$.

$x_{3}{ }^{(\mathrm{s})}: \pm 0.0001$.

$x_{1}: \pm 1.5 \%$ (relative error).

\begin{tabular}{|c|c|}
\hline $\begin{array}{l}\text { Components: } \\
\text { (1) Anthracene; } \mathrm{C}_{14} \mathrm{H}_{10} ;[120-12-7] \\
\text { (2) Cyclohexane; } \mathrm{C}_{6} \mathrm{H}_{12} ;[110-82-7] \\
\text { (3) 2-Propanol; } \mathrm{C}_{3} \mathrm{H}_{8} \mathrm{O} ;[67-63-0] \\
\text { (4) 1-Pentanol; } \mathrm{C}_{5} \mathrm{H}_{12} \mathrm{O} ;[71-41-0]\end{array}$ & $\begin{array}{l}\text { Original Measurements: } \\
{ }^{11} \text { B. A. Martine, B. H. Brooke- } \\
\text { Taylor, and W. E. Acree, Jr., J. } \\
\text { Chem. Eng. Data 53, } 556 \text { (2008). }\end{array}$ \\
\hline $\begin{array}{l}\text { Variables: } \\
T / \mathrm{K}=298.15 \text {; Solvent Composition }\end{array}$ & $\begin{array}{l}\text { Prepared by: } \\
\text { W. E. Acree, Jr. }\end{array}$ \\
\hline
\end{tabular}




\section{Experimental Values}

Solubility of anthracene in cyclohexane +2 -propanol +1 -pentanol mixtures ${ }^{\mathrm{a}}$

\begin{tabular}{lcccc}
\hline \hline$x_{2}{ }^{(\mathrm{s})}$ & $x_{3}{ }^{(\mathrm{s})}$ & $x_{2}$ & $x_{3}$ & $x_{1}$ \\
\hline 0.0000 & 0.0000 & 0.0000 & 0.0000 & 0.001097 \\
1.0000 & 0.0000 & 0.9984 & 0.0000 & 0.001553 \\
0.0000 & 1.0000 & 0.0000 & 0.9996 & 0.000411 \\
0.2848 & 0.4268 & 0.2845 & 0.4263 & 0.001073 \\
0.1425 & 0.1957 & 0.1423 & 0.1955 & 0.001104 \\
0.1253 & 0.7544 & 0.1252 & 0.7539 & 0.000683 \\
0.6658 & 0.1913 & 0.6648 & 0.1910 & 0.001521 \\
0.2271 & 0.3334 & 0.2269 & 0.3330 & 0.001079 \\
0.4469 & 0.3246 & 0.4463 & 0.3242 & 0.001281 \\
0.2019 & 0.5918 & 0.2017 & 0.5913 & 0.000891 \\
0.0950 & 0.2645 & 0.0949 & 0.2642 & 0.001019 \\
0.6679 & 0.1390 & 0.6668 & 0.1388 & 0.001603 \\
0.0818 & 0.7588 & 0.0817 & 0.7583 & 0.000652 \\
0.6495 & 0.2483 & 0.6485 & 0.2479 & 0.001502 \\
0.1918 & 0.1384 & 0.1916 & 0.1382 & 0.001189 \\
0.1664 & 0.7497 & 0.1663 & 0.7492 & 0.000726 \\
0.0995 & 0.4696 & 0.0994 & 0.4692 & 0.000880 \\
0.0959 & 0.5702 & 0.0958 & 0.5697 & 0.000798 \\
0.3794 & 0.1367 & 0.3789 & 0.1365 & 0.001347 \\
0.3352 & 0.5805 & 0.3349 & 0.5799 & 0.000999 \\
0.4857 & 0.1323 & 0.4850 & 0.1321 & 0.001441 \\
0.4239 & 0.4851 & 0.4234 & 0.4845 & 0.001146 \\
\hline
\end{tabular}

${ }^{\mathrm{a}} x_{2}{ }^{(\mathrm{s})}$ : initial mole fraction of component 2 in the ternary solvent mixture, $x_{3}{ }^{(\mathrm{s})}$ : initial mole fraction of component 3 in the ternary solvent mixture; $x_{1}$ : mole fraction solubility of the solute; $x_{2}$ : mole fraction of component 2 in the quaternary solution; $x_{3}$ : mole fraction of component 3 in the quaternary solution.

\section{Auxiliary Information}

\section{Method/Apparatus/Procedure:}

Constant-temperature bath, calorimetric thermometer, and an ultraviolet/ visible spectrophotometer.

Ternary solvent mixtures were prepared by mass. Excess solute and solvent were placed in amber glass bottles and allowed to equilibrate for several days at constant temperature. Attainment of equilibrium was verified by several repetitive measurements and by approaching equilibrium from supersaturation. Aliquots of saturated solutions were transferred through a coarse filter into volumetric flasks, weighed and diluted with methanol. Molar concentrations were determined by spectrophotometric measurements at $356 \mathrm{~nm}$.

\section{Source and Purity of Chemicals:}

(1) $99+\%$, Aldrich Chemical Company, Milwaukee, WI, USA, was recrystallized several times from acetone.

(2) $99 \%$, anhydrous, Aldrich Chemical Company, Milwaukee, WI, USA.

(3) $99+\%$, anhydrous, Aldrich Chemical Company.

(4) $99 \%$, Aldrich Chemical Company.

Components $2-4$ were stored over molecular sieves and distilled shortly before use.

\section{Estimated Error:}

Temperature: $\pm 0.05 \mathrm{~K}$.

$x_{2}{ }^{(\mathrm{s})}: \pm 0.0001$

$x_{3}{ }^{(\mathrm{s})}: \pm 0.0001$.

$x_{1}: \pm 1.5 \%$ (relative error).

\begin{tabular}{ll}
\hline \hline Components: & Original Measurements: \\
(1) Anthracene; $\mathrm{C}_{14} \mathrm{H}_{10} ;[120-12-7]$ & ${ }^{11} \mathrm{~B}$. A. Martine, B. H. Brooke- \\
(2) Cyclohexane; $\mathrm{C}_{6} \mathrm{H}_{12} ;[110-82-7]$ & Taylor, and W. E. Acree, Jr., J. \\
(3) 1-Butanol; $\mathrm{C}_{4} \mathrm{H}_{10} \mathrm{O} ;[71-36-3]$ & Chem. Eng. Data 53, 556 (2008). \\
(4) 1-Pentanol; $\mathrm{C}_{5} \mathrm{H}_{12} \mathrm{O} ;[71-41-0]$ & \\
\hline Variables: & Prepared by: \\
$T / \mathrm{K}=298.15 ;$ Solvent Composition & W. E. Acree, Jr. \\
\hline
\end{tabular}

Experimental Values

Solubility of anthracene in cyclohexane +1 -butanol +1 -pentanol mixtures ${ }^{\mathrm{a}}$

\begin{tabular}{lcccc}
\hline \hline$x_{2}{ }^{(\mathrm{s})}$ & $x_{3}{ }^{(\mathrm{s})}$ & $x_{2}$ & $x_{3}$ & $x_{1}$ \\
\hline 0.0000 & 0.0000 & 0.0000 & 0.0000 & 0.001097 \\
1.0000 & 0.0000 & 0.9984 & 0.0000 & 0.001553 \\
0.0000 & 1.0000 & 0.0000 & 0.9992 & 0.000801 \\
0.3156 & 0.3683 & 0.3152 & 0.3678 & 0.001261 \\
0.1487 & 0.1575 & 0.1485 & 0.1573 & 0.001183 \\
0.1381 & 0.7288 & 0.1380 & 0.7281 & 0.000992 \\
0.6720 & 0.1721 & 0.6709 & 0.1718 & 0.001574 \\
0.2491 & 0.2826 & 0.2488 & 0.2823 & 0.001231 \\
0.4729 & 0.2823 & 0.4722 & 0.2819 & 0.001425 \\
0.2378 & 0.5382 & 0.2375 & 0.5376 & 0.001144 \\
0.1010 & 0.2315 & 0.1008 & 0.2312 & 0.001132 \\
0.6710 & 0.1187 & 0.6699 & 0.1185 & 0.001601 \\
0.0863 & 0.7246 & 0.0862 & 0.7239 & 0.000963 \\
0.6689 & 0.2301 & 0.6679 & 0.2297 & 0.001523 \\
0.2101 & 0.1200 & 0.2098 & 0.1198 & 0.001252 \\
0.1724 & 0.7255 & 0.1722 & 0.7247 & 0.001038 \\
0.0939 & 0.4331 & 0.0938 & 0.4326 & 0.001072 \\
0.0916 & 0.5499 & 0.0915 & 0.5493 & 0.001021 \\
0.3954 & 0.1115 & 0.3948 & 0.1113 & 0.001410 \\
0.3672 & 0.5394 & 0.3667 & 0.5387 & 0.001266 \\
0.4887 & 0.1217 & 0.4880 & 0.1215 & 0.001504 \\
0.4621 & 0.4380 & 0.4615 & 0.4374 & 0.001360 \\
\hline $\mathrm{a}_{x_{2}}{ }^{(\mathrm{s})}$. & initial mole fraction of component2 in the ternary solvent mixture, $x_{3}{ }^{(\mathrm{s})}$.
\end{tabular}

${ }^{a} x_{2}{ }^{(\mathrm{s})}$ : initial mole fraction of component 2 in the ternary solvent mixture, $x_{3}{ }^{(\mathrm{s})}$ : initial mole fraction of component 3 in the ternary solvent mixture; $x_{1}$ : mole fraction solubility of the solute; $x_{2}$ : mole fraction of component 2 in the quaternary solution; $x_{3}$ : mole fraction of component 3 in the quaternary solution.
Information

Method/Apparatus/Procedure:

Constant-temperature bath, calorimetric thermometer, and an ultraviolet/ visible spectrophotometer.

Ternary solvent mixtures were prepared by mass. Excess solute and solvent were placed in amber glass bottles and allowed to equilibrate for several days at constant temperature. Attainment of equilibrium was verified by several repetitive measurements and by approaching equilibrium from supersaturation. Aliquots of saturated solutions were transferred through a coarse filter into volumetric flasks, weighed and diluted with methanol. Molar concentrations were determined by spectrophotometric measurements at $356 \mathrm{~nm}$.

Source and Purity of Chemicals:

(1) $99+\%$, Aldrich Chemical Company, Milwaukee, WI, USA, was recrystallized several times from acetone.

(2) $99 \%$, anhydrous, Aldrich Chemical Company, Milwaukee, WI, USA.

(3) $99+\%$, anhydrous, Aldrich Chemical Company.

(4) $99 \%$, Aldrich Chemical Company.

Components $2-4$ were stored over molecular sieves and distilled shortly before use. 
Estimated Error:

Temperature: $\pm 0.05 \mathrm{~K}$.

$x_{2}{ }^{(\mathrm{s})}: \pm 0.0001$.

$x_{3}{ }^{(\mathrm{s})}: \pm 0.0001$.

$x_{1}: \pm 1.5 \%$ (relative error).

\begin{tabular}{ll}
\hline \hline Components: & Original Measurements: \\
(1) Anthracene; $\mathrm{C}_{14} \mathrm{H}_{10} ;[120-12-7]$ & ${ }^{11}$ B. A. Martine, B. H. Brooke- \\
(2) Cyclohexane; $\mathrm{C}_{6} \mathrm{H}_{12} ;[110-82-7]$ & Taylor, and W. E. Acree, Jr., J. \\
(3) 2-Butanol; $\mathrm{C}_{4} \mathrm{H}_{10} \mathrm{O} ;[78-92-2]$ & Chem. Eng. Data 53, 556 (2008). \\
(4) 1-Pentanol; $\mathrm{C}_{5} \mathrm{H}_{12} \mathrm{O} ;[71-41-0]$ & \\
\hline Variables: & Prepared by: \\
$T / \mathrm{K}=298.15 ;$ Solvent Composition & W. E. Acree, Jr. \\
\hline
\end{tabular}

\section{Experimental Values}

Solubility of anthracene in cyclohexane +2 -butanol +1 -pentanol mixtures ${ }^{\mathrm{a}}$

\begin{tabular}{|c|c|c|c|c|}
\hline$x_{2}{ }^{(\mathrm{s})}$ & $x_{3}{ }^{(\mathrm{s})}$ & $x_{2}$ & $x_{3}$ & $x_{1}$ \\
\hline 0.0000 & 0.0000 & 0.0000 & 0.0000 & 0.001097 \\
\hline 1.0000 & 0.0000 & 0.9984 & 0.0000 & 0.001553 \\
\hline 0.0000 & 1.0000 & 0.0000 & 0.9994 & 0.000585 \\
\hline 0.3165 & 0.3765 & 0.3161 & 0.3761 & 0.001195 \\
\hline 0.1468 & 0.1786 & 0.1466 & 0.1784 & 0.001135 \\
\hline 0.1291 & 0.7324 & 0.1290 & 0.7318 & 0.000836 \\
\hline 0.6818 & 0.1714 & 0.6807 & 0.1711 & 0.001571 \\
\hline 0.2397 & 0.2759 & 0.2394 & 0.2756 & 0.001161 \\
\hline 0.4738 & 0.2824 & 0.4731 & 0.2820 & 0.001388 \\
\hline 0.2268 & 0.5405 & 0.2266 & 0.5399 & 0.001028 \\
\hline 0.0910 & 0.2397 & 0.0909 & 0.2394 & 0.001062 \\
\hline 0.6935 & 0.1179 & 0.6924 & 0.1177 & 0.001607 \\
\hline 0.0954 & 0.7189 & 0.0953 & 0.7183 & 0.000803 \\
\hline 0.6740 & 0.2312 & 0.6730 & 0.2308 & 0.001540 \\
\hline 0.1924 & 0.1212 & 0.1922 & 0.1211 & 0.001203 \\
\hline 0.1782 & 0.7290 & 0.1780 & 0.7284 & 0.000885 \\
\hline 0.0935 & 0.4444 & 0.0934 & 0.4440 & 0.000942 \\
\hline 0.1158 & 0.5243 & 0.1157 & 0.5238 & 0.000915 \\
\hline 0.3968 & 0.1260 & 0.3963 & 0.1258 & 0.001360 \\
\hline 0.3737 & 0.5330 & 0.3733 & 0.5324 & 0.001173 \\
\hline 0.4946 & 0.1183 & 0.4939 & 0.1181 & 0.001454 \\
\hline 0.4723 & 0.4389 & 0.4717 & 0.4383 & 0.001301 \\
\hline
\end{tabular}

${ }^{\mathrm{a}} x_{2}{ }^{(\mathrm{s})}$ : initial mole fraction of component 2 in the ternary solvent mixture, $x_{3}{ }^{(\mathrm{s})}$ : initial mole fraction of component 3 in the ternary solvent mixture; $x_{1}$ : mole fraction solubility of the solute; $x_{2}$ : mole fraction of component 2 in the quaternary solution; $x_{3}$ : mole fraction of component 3 in the quaternary solution.

\section{Auxiliary Information}

\section{Method/Apparatus/Procedure:}

Constant-temperature bath, calorimetric thermometer, and an ultraviolet/ visible spectrophotometer.

Ternary solvent mixtures were prepared by mass. Excess solute and solvent were placed in amber glass bottles and allowed to equilibrate for several days at constant temperature. Attainment of equilibrium was verified by several repetitive measurements and by approaching equilibrium from supersaturation. Aliquots of saturated solutions were transferred through a coarse filter into volumetric flasks, weighed and diluted with methanol. Molar concentrations were determined by spectrophotometric measurements at $356 \mathrm{~nm}$.
Source and Purity of Chemicals:

(1) $99+\%$, Aldrich Chemical Company, Milwaukee, WI, USA, was recrystallized several times from acetone.

(2) $99 \%$, anhydrous, Aldrich Chemical Company, Milwaukee, WI, USA.

(3) $99+\%$, anhydrous, Aldrich Chemical Company.

(4) $99 \%$, Aldrich Chemical Company.

Components 2-4 were stored over molecular sieves and distilled shortly before use.

\section{Estimated Error:}

Temperature: $\pm 0.05 \mathrm{~K}$

$x_{2}{ }^{(s)}: \pm 0.0001$.

$x_{3}{ }^{(\mathrm{s})}: \pm 0.0001$.

$x_{1}: \pm 1.5 \%$ (relative error)

\subsection{Anthracene solubility data in ternary $2,2,4-$ trimethylpentane + alcohol + alcohol solvent mixtures}

\begin{tabular}{ll}
\hline \hline Components: & Original Measurements: \\
(1) Anthracene; $\mathrm{C}_{14} \mathrm{H}_{10} ;[120-12-7]$ & ${ }^{26} \mathrm{~T}$. Deng, S. D. Childress, K. M. \\
(2) 1-Propanol; $\mathrm{C}_{3} \mathrm{H}_{8} \mathrm{O} ;[71-23-8]$ & De Fina, T. L. Sharp, and W. E. \\
(3) 1-Butanol; $\mathrm{C}_{4} \mathrm{H}_{10} \mathrm{O} ;[71-36-3]$ & Acree, Jr., J. Chem. Eng. Data 43, \\
(4) 2,2,4-Trimethylpentane; $\mathrm{C}_{8} \mathrm{H}_{18} ;$ & 1065 (1999). \\
[540-84-1] & \\
\hline Variables: & Prepared by: \\
$T / \mathrm{K}=298.15 ;$ Solvent Composition & W. E. Acree, Jr. \\
\hline
\end{tabular}

\section{Experimental Values}

Solubility of anthracene in 1-propanol + 1-butanol + 2,2,4-trimethylpentane mixtures $^{\mathrm{a}}$

\begin{tabular}{lcccc}
\hline \hline$x_{2}{ }^{(\mathrm{s})}$ & $x_{3}{ }^{(\mathrm{s})}$ & $x_{2}$ & $x_{3}$ & $x_{1}$ \\
\hline 0.0000 & 0.0000 & 0.0000 & 0.0000 & 0.001074 \\
1.0000 & 0.0000 & 0.9994 & 0.0000 & 0.000591 \\
0.0000 & 1.0000 & 0.0000 & 0.9992 & 0.000801 \\
0.4435 & 0.2395 & 0.4431 & 0.2393 & 0.000859 \\
0.1929 & 0.7217 & 0.1927 & 0.7211 & 0.000834 \\
0.3694 & 0.2952 & 0.3691 & 0.2949 & 0.000943 \\
0.3254 & 0.5270 & 0.3251 & 0.5266 & 0.000848 \\
0.8169 & 0.0894 & 0.8163 & 0.0893 & 0.000707 \\
0.7674 & 0.1803 & 0.7669 & 0.1802 & 0.000687 \\
0.2459 & 0.6932 & 0.2457 & 0.6926 & 0.000804 \\
0.1458 & 0.5880 & 0.1457 & 0.5874 & 0.000937 \\
0.4571 & 0.4883 & 0.4568 & 0.4879 & 0.000746 \\
0.5695 & 0.3721 & 0.5691 & 0.3718 & 0.000727 \\
0.2645 & 0.2247 & 0.2642 & 0.2245 & 0.001035 \\
0.7877 & 0.1379 & 0.7871 & 0.1378 & 0.000701 \\
0.6094 & 0.2510 & 0.6089 & 0.2508 & 0.000787 \\
0.1729 & 0.2282 & 0.1727 & 0.2280 & 0.001056 \\
0.1350 & 0.7436 & 0.1349 & 0.7430 & 0.000869 \\
0.3431 & 0.1416 & 0.3427 & 0.1415 & 0.001022 \\
0.1534 & 0.4995 & 0.1532 & 0.4990 & 0.000998 \\
0.5683 & 0.1189 & 0.5678 & 0.1188 & 0.000878 \\
0.6548 & 0.1104 & 0.6543 & 0.1103 & 0.000825 \\
\hline
\end{tabular}

${ }^{\mathrm{a}} x_{2}{ }^{(\mathrm{s})}$ : initial mole fraction of component 2 in the ternary solvent mixture, $x_{3}{ }^{(\mathrm{s})}$ : initial mole fraction of component 3 in the ternary solvent mixture; $x_{1}$ : mole fraction solubility of the solute; $x_{2}$ : mole fraction of component 2 in the quaternary solution; $x_{3}$ : mole fraction of component 3 in the quaternary solution. 


\section{Auxiliary Information}

\section{Method/Apparatus/Procedure:}

Constant-temperature bath, calorimetric thermometer, and an ultraviolet/ visible spectrophotometer.

Ternary solvent mixtures were prepared by mass. Excess solute and solvent were placed in amber glass bottles and allowed to equilibrate for several days at constant temperature. Attainment of equilibrium was verified by several repetitive measurements and by approaching equilibrium from supersaturation. Aliquots of saturated solutions were transferred through a coarse filter into volumetric flasks, weighed and diluted with methanol. Molar concentrations were determined by spectrophotometric measurements at $356 \mathrm{~nm}$.

\section{Source and Purity of Chemicals:}

(1) $99+\%$, Acros Chemical Company, USA, recrystallized three times from acetone.

(2) $99+\%$, anhydrous, Aldrich Chemical Company, Milwaukee, WI, USA.

(3) $99.8 \%$, HPLC grade, Aldrich Chemical Company.

(4) $99.7 \%$, HPLC grade, Aldrich Chemical Company.

Components $2-4$ were stored over molecular sieves and distilled shortly before use.

\section{Estimated Error:}

Temperature: $\pm 0.1 \mathrm{~K}$

$x_{2}{ }^{(\mathrm{s})}: \pm 0.0001$.

$x_{3}{ }^{(\mathrm{s})}: \pm 0.0001$

$x_{1}: \pm 1.5 \%$ (relative error)

\section{Components:}

(1) Anthracene; $\mathrm{C}_{14} \mathrm{H}_{10} ;[120-12-7]$

(2) 1-Propanol; $\mathrm{C}_{3} \mathrm{H}_{8} \mathrm{O}$; [71-23-8]

(3) 2-Butanol; $\mathrm{C}_{4} \mathrm{H}_{10} \mathrm{O}$; [78-92-2]

(4) 2,2,4-Trimethylpentane; $\mathrm{C}_{8} \mathrm{H}_{18}$; [540-84-1]

\begin{tabular}{ll}
\hline Variables: & Prepared by: \\
$T / \mathrm{K}=298.15 ;$ Solvent Composition & W. E. Acree, Jr. \\
\hline
\end{tabular}

\section{Experimental Values}

Solubility of anthracene in 1-propanol +2 -butanol $+2,2,4$-trimethylpentane mixtures $^{\mathrm{a}}$

\begin{tabular}{lcccc}
\hline \hline$x_{2}{ }^{(\mathrm{s})}$ & $x_{3}{ }^{(\mathrm{s})}$ & $x_{2}$ & $x_{3}$ & $x_{1}$ \\
\hline 0.0000 & 0.0000 & 0.0000 & 0.0000 & 0.001074 \\
1.0000 & 0.0000 & 0.9994 & 0.0000 & 0.000591 \\
0.0000 & 1.0000 & 0.0000 & 0.9994 & 0.000585 \\
0.4377 & 0.3595 & 0.4374 & 0.3592 & 0.000781 \\
0.1928 & 0.7220 & 0.1927 & 0.7215 & 0.000675 \\
0.3684 & 0.3005 & 0.3681 & 0.3002 & 0.000881 \\
0.3263 & 0.5277 & 0.3261 & 0.5273 & 0.000725 \\
0.7996 & 0.0900 & 0.7990 & 0.0899 & 0.000697 \\
0.7771 & 0.1745 & 0.7766 & 0.1744 & 0.000636 \\
0.2464 & 0.6966 & 0.2462 & 0.6962 & 0.000646 \\
0.1480 & 0.5903 & 0.1479 & 0.5898 & 0.000845 \\
0.4701 & 0.4770 & 0.4698 & 0.4767 & 0.000637 \\
0.5720 & 0.3736 & 0.5716 & 0.3734 & 0.000630 \\
0.2494 & 0.2078 & 0.2491 & 0.2076 & 0.001017 \\
0.7883 & 0.1377 & 0.7878 & 0.1376 & 0.000665 \\
0.6149 & 0.2466 & 0.6145 & 0.2464 & 0.000721
\end{tabular}

$\begin{array}{lllll}0.1720 & 0.2865 & 0.1718 & 0.2862 & 0.001009 \\ 0.1287 & 0.7485 & 0.1286 & 0.7480 & 0.000718 \\ 0.4497 & 0.1113 & 0.4493 & 0.1112 & 0.000949 \\ 0.1553 & 0.4965 & 0.1552 & 0.4960 & 0.000911 \\ 0.5660 & 0.1153 & 0.5655 & 0.1152 & 0.000874 \\ 0.6568 & 0.1052 & 0.6563 & 0.1051 & 0.000805\end{array}$

${ }^{\mathrm{a}} x_{2}{ }^{(\mathrm{s})}$ : initial mole fraction of component 2 in the ternary solvent mixture, $x_{3}{ }^{(\mathrm{s})}$ : initial mole fraction of component 3 in the ternary solvent mixture; $x_{1}$ : mole fraction solubility of the solute; $x_{2}$ : mole fraction of component 2 in the quaternary solution; $x_{3}$ : mole fraction of component 3 in the quaternary solution.

\section{Method/Apparatus/Procedure:}

Constant-temperature bath, calorimetric thermometer, and an ultraviolet/ visible spectrophotometer.

Ternary solvent mixtures were prepared by mass. Excess solute and solvent were placed in amber glass bottles and allowed to equilibrate for several days at constant temperature. Attainment of equilibrium was verified by several repetitive measurements and by approaching equilibrium from supersaturation. Aliquots of saturated solutions were transferred through a coarse filter into volumetric flasks, weighed and diluted with methanol. Molar concentrations were determined by spectrophotometric measurements at $356 \mathrm{~nm}$.

\section{Source and Purity of Chemicals:}

(1) $99+\%$, Acros Chemical Company, USA, recrystallized three times from acetone.

(2) $99+\%$, anhydrous, Aldrich Chemical Company, Milwaukee, WI, USA.

(3) $99+\%$, anhydrous, Aldrich Chemical Company.

(4) $99.7 \%$, HPLC grade, Aldrich Chemical Company.

Components 2-4 were stored over molecular sieves and distilled shortly before use.

\section{Estimated Error:}

Temperature: $\pm 0.1 \mathrm{~K}$.

$x_{2}{ }^{(\mathrm{s})}: \pm 0.0001$.

$x_{3}{ }^{(\mathrm{s})}: \pm 0.0001$.

$x_{1}: \pm 1.5 \%$ (relative error)

\begin{tabular}{ll}
\hline \hline Components: & Original Measurements: \\
(1) Anthracene; $\mathrm{C}_{14} \mathrm{H}_{10} ;[120-12-7]$ & ${ }^{26} \mathrm{~T}$. Deng, S. D. Childress, K. M. \\
(2) 2-Propanol; $\mathrm{C}_{3} \mathrm{H}_{8} \mathrm{O} ;[67-63-0]$ & De Fina, T. L. Sharp, and W. E. \\
(3) 1-Butanol; $\mathrm{C}_{4} \mathrm{H}_{10} \mathrm{O} ;[71-36-3]$ & Acree, Jr., J. Chem. Eng. Data 43, \\
(4) 2,2,4-Trimethylpentane; $\mathrm{C}_{8} \mathrm{H}_{18} ;$ & 1065 (1999). \\
[540-84-1] & \\
\hline Variables: & Prepared by: \\
T/K = 298.15; Solvent Composition & W. E. Acree, Jr. \\
\hline
\end{tabular}


Experimental Values

Solubility of anthracene in 2-propanol + 1-butanol + 2,2,4-trimethylpentane mixtures $^{\mathrm{a}}$

\begin{tabular}{lcccc}
\hline \hline$x_{2}{ }^{(\mathrm{s})}$ & $x_{3}{ }^{(\mathrm{s})}$ & $x_{2}$ & $x_{3}$ & $x_{1}$ \\
\hline 0.0000 & 0.0000 & 0.0000 & 0.0000 & 0.001074 \\
1.0000 & 0.0000 & 0.9996 & 0.0000 & 0.000411 \\
0.0000 & 1.0000 & 0.0000 & 0.9992 & 0.000801 \\
0.4292 & 0.3609 & 0.4287 & 0.3606 & 0.000790 \\
0.1910 & 0.7235 & 0.1908 & 0.7229 & 0.000794 \\
0.3641 & 0.2998 & 0.3638 & 0.2995 & 0.000883 \\
0.3170 & 0.5335 & 0.3168 & 0.5331 & 0.000786 \\
0.7964 & 0.0964 & 0.7959 & 0.0963 & 0.000579 \\
0.7636 & 0.1840 & 0.7632 & 0.1839 & 0.000545 \\
0.2375 & 0.7068 & 0.2373 & 0.7063 & 0.000758 \\
0.1400 & 0.5964 & 0.1399 & 0.5958 & 0.000933 \\
0.4599 & 0.4855 & 0.4596 & 0.4852 & 0.000661 \\
0.5662 & 0.3817 & 0.5658 & 0.3815 & 0.000623 \\
0.2482 & 0.2120 & 0.2479 & 0.2118 & 0.001015 \\
0.7791 & 0.1406 & 0.7787 & 0.1405 & 0.000562 \\
0.6034 & 0.2523 & 0.6030 & 0.2521 & 0.000678 \\
0.1717 & 0.2768 & 0.1715 & 0.2765 & 0.001051 \\
0.1247 & 0.7546 & 0.1246 & 0.7540 & 0.000850 \\
0.3424 & 0.1350 & 0.3421 & 0.1349 & 0.000967 \\
0.1508 & 0.5030 & 0.1507 & 0.5025 & 0.000979 \\
0.5590 & 0.1166 & 0.5586 & 0.1165 & 0.000802 \\
0.6495 & 0.1082 & 0.6490 & 0.1081 & 0.000720 \\
\hline $\mathrm{a}_{x_{2}}{ }^{(\mathrm{s})}$. initial mole fraction of component 2 in the ternary solvent mixture, $x_{3}^{(\mathrm{s})}$
\end{tabular}

in the ternary solvent mixture, $x_{3}{ }^{(\mathrm{s})}$ : initial mole fraction of component 3 in the ternary solvent mixture; $x_{1}$ : mole fraction solubility of the solute; $x_{2}$ : mole fraction of component 2 in the quaternary solution; $x_{3}$ : mole fraction of component 3 in the quaternary solution.

\section{Auxiliary Information}

\section{Method/Apparatus/Procedure:}

Constant-temperature bath, calorimetric thermometer, and an ultraviolet/ visible spectrophotometer.

Ternary solvent mixtures were prepared by mass. Excess solute and solvent were placed in amber glass bottles and allowed to equilibrate for several days at constant temperature. Attainment of equilibrium was verified by several repetitive measurements and by approaching equilibrium from supersaturation. Aliquots of saturated solutions were transferred through a coarse filter into volumetric flasks, weighed and diluted with methanol. Molar concentrations were determined by spectrophotometric measurements at $356 \mathrm{~nm}$.

\section{Source and Purity of Chemicals:}

(1) $99+\%$, Acros Chemical Company, USA, recrystallized three times from acetone.

(2) $99+\%$, anhydrous, Aldrich Chemical Company, Milwaukee, WI, USA.

(3) $99.8 \%$, HPLC grade, Aldrich Chemical Company.

(4) $99.7 \%$, HPLC grade, Aldrich Chemical Company.

Components 2-4 were stored over molecular sieves and distilled shortly before use.

\section{Estimated Error:}

Temperature: $\pm 0.1 \mathrm{~K}$.

$x_{2}^{(\mathrm{s})}: \pm 0.0001$.

$x_{3}{ }^{(\mathrm{s})}: \pm 0.0001$.

$x_{1}: \pm 1.5 \%$ (relative error).

\begin{tabular}{|c|c|}
\hline $\begin{array}{l}\text { Components: } \\
\text { (1) Anthracene; } \mathrm{C}_{14} \mathrm{H}_{10} ;[120-12-7] \\
\text { (2) 2-Propanol; } \mathrm{C}_{3} \mathrm{H}_{8} \mathrm{O} ;[67-63-0] \\
\text { (3) 2-Butanol; } \mathrm{C}_{4} \mathrm{H}_{10} \mathrm{O} ;[78-92-2] \\
\text { (4) 2,2,4-Trimethylpentane; } \mathrm{C}_{8} \mathrm{H}_{18} \text {; } \\
\text { [540-84-1] }\end{array}$ & $\begin{array}{l}\text { Original Measurements: } \\
{ }^{26} \text { T. Deng, S. D. Childress, K. M. } \\
\text { De Fina, T. L. Sharp, and W. E. } \\
\text { Acree, Jr., J. Chem. Eng. Data 43, } \\
1065 \text { (1999). }\end{array}$ \\
\hline $\begin{array}{l}\text { Variables: } \\
T / \mathrm{K}=298.15 ; \text { Solvent Composition }\end{array}$ & $\begin{array}{l}\text { Prepared by: } \\
\text { W. E. Acree, Jr. }\end{array}$ \\
\hline
\end{tabular}

Experimental Values

Solubility of anthracene in 2-propanol + 2-butanol $+2,2,4$-trimethylpentane mixtures $^{\text {a }}$

\begin{tabular}{lcccc}
\hline \hline$x_{2}{ }^{(\mathrm{s})}$ & $x_{3}{ }^{(\mathrm{s})}$ & $x_{2}$ & $x_{3}$ & $x_{1}$ \\
\hline 0.0000 & 0.0000 & 0.0000 & 0.0000 & 0.001074 \\
1.0000 & 0.0000 & 0.9996 & 0.0000 & 0.000411 \\
0.0000 & 1.0000 & 0.0000 & 0.9994 & 0.000585 \\
0.4326 & 0.3660 & 0.4323 & 0.3657 & 0.000709 \\
0.1880 & 0.7267 & 0.1879 & 0.7262 & 0.000651 \\
0.3650 & 0.3021 & 0.3647 & 0.3018 & 0.000833 \\
0.3220 & 0.5324 & 0.3218 & 0.5320 & 0.000674 \\
0.7965 & 0.0989 & 0.7961 & 0.0988 & 0.000557 \\
0.7605 & 0.1861 & 0.7601 & 0.1860 & 0.000514 \\
0.2462 & 0.6990 & 0.2460 & 0.6986 & 0.000611 \\
0.1475 & 0.5947 & 0.1474 & 0.5942 & 0.000822 \\
0.4665 & 0.4786 & 0.4662 & 0.4783 & 0.000561 \\
0.5636 & 0.3803 & 0.5633 & 0.3801 & 0.000552 \\
0.2566 & 0.2110 & 0.2563 & 0.2108 & 0.000977 \\
0.7813 & 0.1414 & 0.7809 & 0.1413 & 0.000532 \\
0.6075 & 0.2552 & 0.6071 & 0.2550 & 0.000621 \\
0.1760 & 0.2841 & 0.1758 & 0.2838 & 0.001016 \\
0.1317 & 0.7471 & 0.1316 & 0.7466 & 0.000702 \\
0.3232 & 0.1402 & 0.3229 & 0.1401 & 0.000966 \\
0.1516 & 0.4991 & 0.1515 & 0.4986 & 0.000904 \\
0.5605 & 0.1256 & 0.5601 & 0.1255 & 0.000778 \\
0.6477 & 0.1152 & 0.6472 & 0.1151 & 0.000703 \\
\hline${ }^{a} x_{2}{ }^{(s)}:$ initial mole fraction of component 2 in the ternary solvent mixture, $x_{3}^{(\mathrm{s})}$ : \\
initial mole fraction of component 3 in the ternary solvent mixture; $x_{1}:$ mole \\
fraction solubility of the solute; $x_{2}:$ mole fraction of component 2 in the \\
quaternary solution $; x_{3}:$ mole fraction of component 3 in the quaternary \\
solution. & & & & \\
& & & &
\end{tabular}

\section{Auxiliary Information}

Method/Apparatus/Procedure:

Constant-temperature bath, calorimetric thermometer, and an ultraviolet/ visible spectrophotometer.

Ternary solvent mixtures were prepared by mass. Excess solute and solvent were placed in amber glass bottles and allowed to equilibrate for several days at constant temperature. Attainment of equilibrium was verified by several repetitive measurements and by approaching equilibrium from supersaturation. Aliquots of saturated solutions were transferred through a coarse filter into volumetric flasks, weighed and diluted with methanol. Molar concentrations were determined by spectrophotometric measurements at $356 \mathrm{~nm}$.

Source and Purity of Chemicals:

(1) $99+\%$, Acros Chemical Company, USA, recrystallized three times from acetone.

(2) $99+\%$, anhydrous, Aldrich Chemical Company, Milwaukee, WI, USA.

(3) $99+\%$, anhydrous, Aldrich Chemical Company.

(4) $99.7 \%$, HPLC grade, Aldrich Chemical Company.

Components $2-4$ were stored over molecular sieves and distilled shortly before use. 
Estimated Error:

Temperature: $\pm 0.1 \mathrm{~K}$

$x_{2}^{(\mathrm{s})}: \pm 0.0001$.

$x_{3}{ }^{(\mathrm{s})}: \pm 0.0001$.

$x_{1}: \pm 1.5 \%$ (relative error)

\begin{tabular}{|c|c|}
\hline $\begin{array}{l}\text { Components: } \\
\text { (1) Anthracene; } \mathrm{C}_{14} \mathrm{H}_{10} ;[120-12-7] \\
\text { (2) 2,2,4-Trimethylpentane; } \mathrm{C}_{8} \mathrm{H}_{18} \text {; } \\
\text { [540-84-1] } \\
\text { (3) 1-Propanol; } \mathrm{C}_{3} \mathrm{H}_{8} \mathrm{O} ;[71-23-8] \\
\text { (4) 1-Pentanol; } \mathrm{C}_{5} \mathrm{H}_{12} \mathrm{O} ;[71-41-0]\end{array}$ & $\begin{array}{l}\text { Original Measurements: } \\
{ }^{9} \text { B. H. Blake-Taylor, B. A. } \\
\text { Martine, and W. E. Acree, Jr., J. } \\
\text { Chem. Eng. Data 53, } 970 \text { (2008). }\end{array}$ \\
\hline $\begin{array}{l}\text { Variables: } \\
T / \mathrm{K}=298.15 \text {; Solvent Composition }\end{array}$ & $\begin{array}{l}\text { Prepared by: } \\
\text { W. E. Acree, Jr. }\end{array}$ \\
\hline
\end{tabular}

\section{Experimental Values}

Solubility of anthracene in 2,2,4-trimethylpentane + 1-propanol + 1-pentanol mixtures $^{\mathrm{a}}$

\begin{tabular}{lcccc}
\hline \hline$x_{2}{ }^{(\mathrm{s})}$ & $x_{3}{ }^{(\mathrm{s})}$ & $x_{2}$ & $x_{3}$ & $x_{1}$ \\
\hline 0.0000 & 0.0000 & 0.0000 & 0.0000 & 0.001097 \\
1.0000 & 0.0000 & 0.9989 & 0.0000 & 0.001074 \\
0.0000 & 1.0000 & 0.0000 & 0.9994 & 0.000591 \\
0.2062 & 0.4675 & 0.2060 & 0.4670 & 0.000975 \\
0.0995 & 0.2209 & 0.0994 & 0.2207 & 0.001044 \\
0.0889 & 0.7856 & 0.0888 & 0.7850 & 0.000756 \\
0.5354 & 0.2836 & 0.5348 & 0.2833 & 0.001106 \\
0.1602 & 0.3608 & 0.1600 & 0.3604 & 0.001001 \\
0.3472 & 0.3838 & 0.3468 & 0.3834 & 0.001032 \\
0.1410 & 0.6330 & 0.1409 & 0.6325 & 0.000859 \\
0.0631 & 0.2794 & 0.0630 & 0.2791 & 0.000996 \\
0.5647 & 0.1917 & 0.5641 & 0.1915 & 0.001140 \\
0.0621 & 0.7839 & 0.0621 & 0.7833 & 0.000736 \\
0.5304 & 0.3426 & 0.5298 & 0.3422 & 0.001082 \\
0.1330 & 0.1575 & 0.1329 & 0.1573 & 0.001084 \\
0.1035 & 0.8105 & 0.1034 & 0.8099 & 0.000752 \\
0.0720 & 0.4995 & 0.0719 & 0.4991 & 0.000898 \\
0.0633 & 0.5970 & 0.0632 & 0.5965 & 0.000841 \\
0.2967 & 0.1588 & 0.2964 & 0.1586 & 0.001140 \\
0.2383 & 0.6691 & 0.2381 & 0.6685 & 0.000870 \\
0.3707 & 0.1823 & 0.3703 & 0.1821 & 0.001152 \\
0.3336 & 0.5642 & 0.3333 & 0.5637 & 0.000941 \\
\hline${ }^{a} x_{2}{ }^{\left({ }^{s}\right)}$ initial mole fraction of component 2 in the ternary solvent mixture, $x_{3}{ }^{(\mathrm{s})}$ : \\
initial mole fraction of component 3 in the ternary solvent mixture; $x_{1}:$ mole \\
fraction solubility of the solute; $x_{2}:$ mole fraction of component 2 in the \\
quaternary solution; $x_{3}:$ mole fraction of component 3 in the quaternary \\
solution. & & & & \\
& & & &
\end{tabular}

\section{Auxiliary Information}

\section{Method/Apparatus/Procedure:}

Constant-temperature bath, calorimetric thermometer, and an ultraviolet/ visible spectrophotometer.

Ternary solvent mixtures were prepared by mass. Excess solute and solvent were placed in amber glass bottles and allowed to equilibrate for several days at constant temperature. Attainment of equilibrium was verified by several repetitive measurements and by approaching equilibrium from supersaturation. Aliquots of saturated solutions were transferred through a coarse filter into volumetric flasks, weighed and diluted with methanol. Molar concentrations were determined by spectrophotometric measurements at $356 \mathrm{~nm}$.

Source and Purity of Chemicals:

(1) $99+\%$, Aldrich Chemical Company, Milwaukee, WI, USA, was recrystallized several times from acetone.

(2) $99.8 \%$, anhydrous, Aldrich Chemical Company.

(3) $99+\%$, anhydrous, Aldrich Chemical Company.

(4) $99 \%$, Aldrich Chemical Company.

Components 2-4 were stored over molecular sieves and distilled shortly before use.

\section{Estimated Error:}

Temperature: $\pm 0.05 \mathrm{~K}$

$x_{2}{ }^{(s)}: \pm 0.0001$.

$x_{3}{ }^{(\mathrm{s})}: \pm 0.0001$.

$x_{1}: \pm 1.5 \%$ (relative error).

\section{Components:}

(1) Anthracene; $\mathrm{C}_{14} \mathrm{H}_{10} ;[120-12-7]$

(2) 2,2,4-Trimethylpentane; $\mathrm{C}_{8} \mathrm{H}_{18}$; [540-84-1]

(3) 2-Propanol; $\mathrm{C}_{3} \mathrm{H}_{8} \mathrm{O}$; [67-63-0]

(4) 1-Pentanol; $\mathrm{C}_{5} \mathrm{H}_{12} \mathrm{O} ;[$ [71-41-0]

\begin{tabular}{ll}
\hline Variables: & Prepared by: \\
$T / \mathrm{K}=298.15 ;$ Solvent Composition & W. E. Acree, Jr. \\
\hline
\end{tabular}

Experimental Values

Solubility of anthracene in 2,2,4-trimethylpentane + 2-propanol + 1-pentanol mixtures $^{\mathrm{a}}$

\begin{tabular}{|c|c|c|c|c|}
\hline$x_{2}{ }^{(s)}$ & $x_{3}{ }^{(s)}$ & $x_{2}$ & $x_{3}$ & $x_{1}$ \\
\hline 0.0000 & 0.0000 & 0.0000 & 0.0000 & 0.001097 \\
\hline 1.0000 & 0.0000 & 0.9989 & 0.0000 & 0.001074 \\
\hline 0.0000 & 1.0000 & 0.0000 & 0.9996 & 0.000411 \\
\hline 0.2129 & 0.4662 & 0.2127 & 0.4658 & 0.000887 \\
\hline 0.0763 & 0.2307 & 0.0762 & 0.2305 & 0.000983 \\
\hline 0.0903 & 0.7414 & 0.0902 & 0.7409 & 0.000618 \\
\hline 0.5654 & 0.2600 & 0.5648 & 0.2597 & 0.001062 \\
\hline 0.1634 & 0.3432 & 0.1632 & 0.3429 & 0.000943 \\
\hline 0.3453 & 0.3684 & 0.3450 & 0.3680 & 0.000991 \\
\hline 0.1437 & 0.6294 & 0.1436 & 0.6289 & 0.000746 \\
\hline 0.0592 & 0.2747 & 0.0591 & 0.2744 & 0.000943 \\
\hline 0.5535 & 0.2047 & 0.5529 & 0.2045 & 0.001122 \\
\hline 0.0595 & 0.7832 & 0.0595 & 0.7827 & 0.000590 \\
\hline 0.5281 & 0.3511 & 0.5276 & 0.3507 & 0.001027 \\
\hline 0.1317 & 0.1484 & 0.1315 & 0.1482 & 0.001058 \\
\hline 0.1080 & 0.8053 & 0.1079 & 0.8048 & 0.000604 \\
\hline 0.0581 & 0.4938 & 0.0581 & 0.4934 & 0.000795 \\
\hline 0.0611 & 0.5983 & 0.0611 & 0.5979 & 0.000726 \\
\hline 0.2812 & 0.1550 & 0.2809 & 0.1548 & 0.001113 \\
\hline 0.2375 & 0.6561 & 0.2373 & 0.6556 & 0.000761 \\
\hline 0.3571 & 0.1912 & 0.3567 & 0.1910 & 0.001115 \\
\hline 0.3197 & 0.5670 & 0.3194 & 0.5665 & 0.000843 \\
\hline
\end{tabular}

${ }^{\mathrm{a}} x_{2}{ }^{(\mathrm{s})}$ : initial mole fraction of component 2 in the ternary solvent mixture, $x_{3}{ }^{(\mathrm{s})}$ : initial mole fraction of component 3 in the ternary solvent mixture; $x_{1}$ : mole fraction solubility of the solute; $x_{2}$ : mole fraction of component 2 in the quaternary solution; $x_{3}$ : mole fraction of component 3 in the quaternary solution. 


\section{Auxiliary Information}

Method/Apparatus/Procedure:

Constant-temperature bath, calorimetric thermometer, and an ultraviolet/ visible spectrophotometer.

Ternary solvent mixtures were prepared by mass. Excess solute and solvent were placed in amber glass bottles and allowed to equilibrate for several days at constant temperature. Attainment of equilibrium was verified by several repetitive measurements and by approaching equilibrium from supersaturation. Aliquots of saturated solutions were transferred through a coarse filter into volumetric flasks, weighed and diluted with methanol. Molar concentrations were determined by spectrophotometric measurements at $356 \mathrm{~nm}$.

\section{Source and Purity of Chemicals:}

(1) $99+\%$, Aldrich Chemical Company, Milwaukee, WI, USA, was

recrystallized several times from acetone.

(2) $99.8 \%$, anhydrous, Aldrich Chemical Company.

(3) $99+\%$, anhydrous, Aldrich Chemical Company.

(4) $99 \%$, Aldrich Chemical Company.

Components 2-4 were stored over molecular sieves and distilled shortly before use.

\section{Estimated Error:}

Temperature: $\pm 0.05 \mathrm{~K}$.

$x_{2}{ }^{(\mathrm{s})}: \pm 0.0001$.

$x_{3}{ }^{(\mathrm{s})}: \pm 0.0001$

$x_{1}: \pm 1.5 \%$ (relative error).

\section{Components:}

(1) Anthracene; $\mathrm{C}_{14} \mathrm{H}_{10}$; [120-12-7]

(2) 2,2,4-Trimethylpentane; $\mathrm{C}_{8} \mathrm{H}_{18}$;

[540-84-1]

(3) 1-Butanol; $\mathrm{C}_{4} \mathrm{H}_{10} \mathrm{O}$; [71-36-3]

(4) 1-Pentanol; $\mathrm{C}_{5} \mathrm{H}_{12} \mathrm{O}$; [71-41-0]

\section{Variables:}

$T / \mathrm{K}=298.15$; Solvent Composition

Prepared by:

W. E. Acree, Jr.

\section{Experimental Values}

Solubility of anthracene in 2,2,4-trimethylpentane + 1-butanol +1 -pentanol mixtures $^{\mathrm{a}}$

\begin{tabular}{lcccc}
\hline \hline$x_{2}{ }^{(\mathrm{s})}$ & $x_{3}{ }^{(\mathrm{s})}$ & $x_{2}$ & $x_{3}$ & $x_{1}$ \\
\hline 0.0000 & 0.0000 & 0.0000 & 0.0000 & 0.001097 \\
1.0000 & 0.0000 & 0.9989 & 0.0000 & 0.001074 \\
0.0000 & 1.0000 & 0.0000 & 0.9992 & 0.000801 \\
0.2318 & 0.4236 & 0.2316 & 0.4231 & 0.001069 \\
0.1092 & 0.1894 & 0.1091 & 0.1892 & 0.001107 \\
0.0894 & 0.7683 & 0.0893 & 0.7676 & 0.000919 \\
0.5754 & 0.2307 & 0.5747 & 0.2304 & 0.001183 \\
0.1679 & 0.3258 & 0.1677 & 0.3254 & 0.001080 \\
0.3728 & 0.3505 & 0.3724 & 0.3501 & 0.001133 \\
0.1641 & 0.5876 & 0.1639 & 0.5870 & 0.001001 \\
0.0769 & 0.2522 & 0.0768 & 0.2519 & 0.001071 \\
0.5933 & 0.1446 & 0.5926 & 0.1444 & 0.001231 \\
0.1288 & 0.7067 & 0.1287 & 0.7060 & 0.000956 \\
0.5745 & 0.2912 & 0.5738 & 0.2909 & 0.001167
\end{tabular}

$\begin{array}{lllll}0.1455 & 0.1277 & 0.1453 & 0.1276 & 0.001128 \\ 0.1337 & 0.7689 & 0.1336 & 0.7682 & 0.000931 \\ 0.0747 & 0.4475 & 0.0746 & 0.4470 & 0.001007 \\ 0.0748 & 0.5490 & 0.0747 & 0.5485 & 0.000983 \\ 0.2977 & 0.1361 & 0.2973 & 0.1359 & 0.001176 \\ 0.2799 & 0.6186 & 0.2796 & 0.6180 & 0.001013 \\ 0.3827 & 0.1435 & 0.3822 & 0.1433 & 0.001202 \\ 0.3694 & 0.5121 & 0.3690 & 0.5116 & 0.001069\end{array}$

${ }^{\mathrm{a}} x_{2}{ }^{(\mathrm{s})}$ : initial mole fraction of component 2 in the ternary solvent mixture, $x_{3}{ }^{(\mathrm{s})}$ : initial mole fraction of component 3 in the ternary solvent mixture; $x_{1}$ : mole fraction solubility of the solute; $x_{2}$ : mole fraction of component 2 in the quaternary solution; $x_{3}$ : mole fraction of component 3 in the quaternary solution.

\section{Auxiliary Information}

\section{Method/Apparatus/Procedure:}

Constant-temperature bath, calorimetric thermometer, and an ultraviolet/ visible spectrophotometer.

Ternary solvent mixtures were prepared by mass. Excess solute and solvent were placed in amber glass bottles and allowed to equilibrate for several days at constant temperature. Attainment of equilibrium was verified by several repetitive measurements and by approaching equilibrium from supersaturation. Aliquots of saturated solutions were transferred through a coarse filter into volumetric flasks, weighed and diluted with methanol. Molar concentrations were determined by spectrophotometric measurements at $356 \mathrm{~nm}$.

Source and Purity of Chemicals:

(1) $99+\%$, Aldrich Chemical Company, Milwaukee, WI, USA, was recrystallized several times from acetone.

(2) $99.8 \%$, anhydrous, Aldrich Chemical Company.

(3) $99+\%$, anhydrous, Aldrich Chemical Company.

(4) $99 \%$, Aldrich Chemical Company.

Components 2-4 were stored over molecular sieves and distilled shortly before use.

Estimated Error:

Temperature: $\pm 0.05 \mathrm{~K}$.

$x_{2}{ }^{(\mathrm{s})}: \pm 0.0001$.

$x_{3}{ }^{(\mathrm{s})}: \pm 0.0001$.

$x_{1}: \pm 1.5 \%$ (relative error)

\begin{tabular}{|c|c|}
\hline $\begin{array}{l}\text { Components: } \\
\text { (1) Anthracene; } \mathrm{C}_{14} \mathrm{H}_{10} ;[120-12-7] \\
\text { (2) } 2,2,4 \text {-Trimethylpentane; } \mathrm{C}_{8} \mathrm{H}_{18} ; \\
\text { [540-84-1] } \\
\text { (3) 2-Butanol; } \mathrm{C}_{4} \mathrm{H}_{10} \mathrm{O} ;[78-92-2] \\
\text { (4) } 1 \text {-Pentanol; } \mathrm{C}_{5} \mathrm{H}_{12} \mathrm{O} ;[71-41-0]\end{array}$ & $\begin{array}{l}\text { Original Measurements: } \\
{ }^{9} \text { B. H. Blake-Taylor, B. A. } \\
\text { Martine, and W. E. Acree, Jr., J. } \\
\text { Chem. Eng. Data 53, } 970 \text { (2008). }\end{array}$ \\
\hline $\begin{array}{l}\text { Variables: } \\
T / \mathrm{K}=298.15 ; \text { Solvent Composition }\end{array}$ & $\begin{array}{l}\text { Prepared by: } \\
\text { W. E. Acree, Jr. }\end{array}$ \\
\hline
\end{tabular}


Experimental Values

Solubility of anthracene in 2,2,4-trimethylpentane +2 -butanol +1 -pentanol mixtures $^{\mathrm{a}}$

\begin{tabular}{lcccc}
\hline \hline$x_{2}{ }^{(\mathrm{s})}$ & $x_{3}{ }^{(\mathrm{s})}$ & $x_{2}$ & $x_{3}$ & $x_{1}$ \\
\hline 0.0000 & 0.0000 & 0.0000 & 0.0000 & 0.001097 \\
1.0000 & 0.0000 & 0.9989 & 0.0000 & 0.001074 \\
0.0000 & 1.0000 & 0.0000 & 0.9994 & 0.000585 \\
0.2273 & 0.4253 & 0.2271 & 0.4249 & 0.000995 \\
0.1036 & 0.1896 & 0.1035 & 0.1894 & 0.001051 \\
0.0831 & 0.7698 & 0.0830 & 0.7692 & 0.000749 \\
0.5590 & 0.2369 & 0.5584 & 0.2366 & 0.001145 \\
0.1736 & 0.3061 & 0.1734 & 0.3058 & 0.001026 \\
0.3658 & 0.3502 & 0.3654 & 0.3498 & 0.001079 \\
0.1670 & 0.5880 & 0.1669 & 0.5875 & 0.000896 \\
0.0744 & 0.2269 & 0.0743 & 0.2267 & 0.001021 \\
0.5874 & 0.1584 & 0.5867 & 0.1582 & 0.001200 \\
0.0635 & 0.7470 & 0.0635 & 0.7464 & 0.000748 \\
0.5731 & 0.3149 & 0.5725 & 0.3145 & 0.001124 \\
0.1368 & 0.1326 & 0.1366 & 0.1325 & 0.001107 \\
0.1290 & 0.7816 & 0.1289 & 0.7810 & 0.000779 \\
0.0709 & 0.4424 & 0.0708 & 0.4420 & 0.000910 \\
0.0664 & 0.5627 & 0.0663 & 0.5622 & 0.000842 \\
0.2949 & 0.1525 & 0.2946 & 0.1523 & 0.001153 \\
0.2792 & 0.6110 & 0.2789 & 0.6104 & 0.000942 \\
0.3640 & 0.1629 & 0.3636 & 0.1627 & 0.001128 \\
0.3643 & 0.5234 & 0.3639 & 0.5229 & 0.001012 \\
\hline${ }^{a}{ }_{2}{ }^{(s)}:$ initial mole fraction of component 2 in the ternary solvent mixture, $x_{3}{ }^{(\mathrm{s})}$ : \\
initial mole fraction of component 3 in the ternary solvent mixture; $x_{1}:$ mole \\
fraction solubility of the solute; $x_{2}:$ mole fraction of component 2 in the \\
quaternary solution $; x_{3}:$ mole fraction of component 3 in the quaternary \\
solution. & & & & \\
& & & &
\end{tabular}

\section{Auxiliary Information}

\section{Method/Apparatus/Procedure:}

Constant-temperature bath, calorimetric thermometer, and an ultraviolet/ visible spectrophotometer.

Ternary solvent mixtures were prepared by mass. Excess solute and solvent were placed in amber glass bottles and allowed to equilibrate for several days at constant temperature. Attainment of equilibrium was verified by several repetitive measurements and by approaching equilibrium from supersaturation. Aliquots of saturated solutions were transferred through a coarse filter into volumetric flasks, weighed and diluted with methanol. Molar concentrations were determined by spectrophotometric measurements at $356 \mathrm{~nm}$.

\section{Source and Purity of Chemicals:}

(1) $99+\%$, Aldrich Chemical Company, Milwaukee, WI, USA, was recrystallized several times from acetone.

(2) $99.8 \%$, anhydrous, Aldrich Chemical Company.

(3) $99+\%$, anhydrous, Aldrich Chemical Company.

(4) $99 \%$, Aldrich Chemical Company.

Components $2-4$ were stored over molecular sieves and distilled shortly before use.

\section{Estimated Error:}

Temperature: $\pm 0.05 \mathrm{~K}$.

$x_{2}^{(\mathrm{s})}: \pm 0.0001$.

$x_{3}{ }^{(\mathrm{s})}: \pm 0.0001$.

$x_{1}: \pm 1.5 \%$ (relative error)

\section{Solubility of Anthracene in Ternary Alkane + Ether + Alcohol Solvent Mixtures}

\subsection{Critical evaluation of experimental solubility data}

Pribyla et al. ${ }^{12-15,17-21}$ reported the solubility of anthracene in ternary alkane $+1,1^{\prime}$-oxybisbutane + alcohol, alkane +2 methoxy-2-methylpropane + alcohol, and alkane +1 ,4-dioxane + alcohol solvent mixtures at $298.15 \mathrm{~K}$. Three alkane cosolvents: heptane (a linear alkane), cyclohexane (a cycloalkane), and 2,2,4-trimethylpentane (a highly branched alkane) and five alcohol cosolvents (1-propanol, 2-propanol, 1-butanol, 2-butanol, and 2-methyl-1-propanol) were studied. There has only been a single experimental determination for the solubility of anthracene in each of the ternary solvent systems studied. In the absence of replicate independent measurements, the experimental values were evaluated by curve-fitting the measured solubility data for the respective sub-binary solvent systems to the Combined NIBS/Redlich-Kister equation, and then using Eq. (2) to estimate the mole fraction solubility of anthracene in ternary alkane + ether + alcohol solvent mixtures.

The calculated $S_{23, i}$ parameters for all of the contributing binary alkane + ether, alkane + alcohol, and ether + alcohol solvent systems studied are summarized in Table 1, along with the average absolute deviation between the experimental and back-calculated mole fraction solubilities. The predictive ability of Eq. (2) is given in Table 5 for the 45 different ternary alkane + ether + alcohol solvent systems. Examination of the numerical entries in the last column of Table 5 reveals that Eq. (2) does provide a very accurate mathematical representation for how the measured mole fraction solubilities vary with binary solvent composition. Average percent deviations range from $1.1 \%$ to $5.4 \%$, suggesting that there are no obvious outliers in an individual ternary solvent data set. The larger deviations were noted in the ternary solvent mixtures containing 1,4-dioxane, where the observed solubility exhibits up to a 20 -fold range in mole fraction solubility. The solubility of anthracene in 1,4-dioxane $\left(x_{1}=0.008329\right)$ is considerably larger than in the five alcohol cosolvents $\left(x_{1}=0.000411\right.$ in 2propanol, $x_{1}=0.000591$ in 1-propanol, $x_{1}=0.000801$ in 1butanol, $x_{1}=0.000585$ in 2-butanol, and $x_{1}=0.000470$ in 2methyl-1-propanol). None of the experimental data points was flagged as an outlier.

The experimental anthracene solubility data for ternary $1,1^{\prime}$ oxybisbutane + alkane + alcohol, 2-methoxy-2-methylpropane + alkane + alcohol, and 1,4-dioxane + alkane + alcohol solvent mixtures are given in Secs. 4.2-4.4. 
TABLE 5. Summarized comparison between experimental solubilities of anthracene in ternary alkane + ether + alcohol solvent mixtures and predicted values based on Eq. (2)

\begin{tabular}{|c|c|}
\hline Ternary solvent mixture & $\%$ Dev. $^{\mathrm{a}}$ \\
\hline $1,1^{\prime}$-Oxybisbutane + heptane +1 -propanol & 1.78 \\
\hline 1,1'-Oxybisbutane + heptane +2 -propanol & 1.37 \\
\hline 1,1'-Oxybisbutane + heptane +1 -butanol & 1.70 \\
\hline 1,1'-Oxybisbutane + heptane +2 -butanol & 1.22 \\
\hline 1,1'-Oxybisbutane + heptane +2 -methyl-1-propanol & 1.65 \\
\hline 1,1'-Oxybisbutane + cyclohexane +1 -propanol & 1.68 \\
\hline 1,1'-Oxybisbutane + cyclohexane +2 -propanol & 1.16 \\
\hline 1,1'-Oxybisbutane + cyclohexane +1 -butanol & 1.94 \\
\hline $1,1^{\prime}$-Oxybisbutane + cyclohexane +2 -butanol & 1.07 \\
\hline 1,1'-Oxybisbutane + cyclohexane +2 -methyl-1-propanol & 1.77 \\
\hline 1,1'-Oxybisbutane $+2,2,4$-trimethylpentane +1 -propanol & 1.71 \\
\hline 1,1'-Oxybisbutane $+2,2,4$-trimethylpentane + 2-propanol & 1.08 \\
\hline 1,1'-Oxybisbutane $+2,2,4$-trimethylpentane +1 -butanol & 1.39 \\
\hline 1,1'-Oxybisbutane $+2,2,4$-trimethylpentane +2 -butanol & 1.29 \\
\hline $\begin{array}{l}\text { 1,1'-Oxybisbutane }+2,2,4 \text {-trimethylpentane }+2 \text {-methyl-1- } \\
\text { propanol }\end{array}$ & 1.45 \\
\hline 2-Methoxy-2-methylpropane + heptane +1 -propanol & 1.85 \\
\hline 2-Methoxy-2-methylpropane + heptane +2 -propanol & 1.60 \\
\hline 2-Methoxy-2-methylpropane + heptane +1 -butanol & 1.92 \\
\hline 2-Methoxy-2-methylpropane + heptane +2 -butanol & 1.16 \\
\hline 2-Methoxy-2-methylpropane + heptane + 2-methyl-1-propanol & 1.60 \\
\hline 2-Methoxy-2-methylpropane + cyclohexane + 1-propanol & 1.70 \\
\hline 2-Methoxy-2-methylpropane + cyclohexane +2 -propanol & 1.66 \\
\hline 2-Methoxy-2-methylpropane + cyclohexane +1 -butanol & 1.73 \\
\hline 2-Methoxy-2-methylpropane + cyclohexane +2 -butanol & 1.78 \\
\hline $\begin{array}{l}\text { 2-Methoxy-2-methylpropane + cyclohexane }+2 \text {-methyl-1- } \\
\text { propanol }\end{array}$ & 1.63 \\
\hline $\begin{array}{l}\text { 2-Methoxy-2-methylpropane }+ \text { 2,2,4-trimethylpentane } \\
+1 \text {-propanol }\end{array}$ & 1.60 \\
\hline $\begin{array}{l}\text { 2-Methoxy-2-methylpropane }+ \text { 2,2,4-trimethylpentane } \\
+2 \text {-propanol }\end{array}$ & 1.61 \\
\hline $\begin{array}{l}\text { 2-Methoxy-2-methylpropane }+ \text { 2,2,4-trimethylpentane } \\
+1 \text {-butanol }\end{array}$ & 1.93 \\
\hline $\begin{array}{l}\text { 2-Methoxy-2-methylpropane }+ \text { 2,2,4-trimethylpentane } \\
+ \text { 2-butanol }\end{array}$ & 1.63 \\
\hline $\begin{array}{l}\text { 2-Methoxy-2-methylpropane }+2,2,4 \text {-trimethylpentane } \\
+2 \text {-methyl-1-propanol }\end{array}$ & 1.68 \\
\hline 1,4-Dioxane + heptane + 1-propanol & 4.02 \\
\hline 1,4-Dioxane + heptane +2 -propanol & 4.38 \\
\hline 1,4-Dioxane + heptane + 1-butanol & 3.53 \\
\hline 1,4-Dioxane + heptane + 2-butanol & 3.41 \\
\hline 1,4-Dioxane + heptane +2 -methyl-1-propanol & 3.22 \\
\hline 1,4-Dioxane + cyclohexane + 1-propanol & 5.13 \\
\hline 1,4-Dioxane + cyclohexane + 2-propanol & 4.62 \\
\hline 1,4-Dioxane + cyclohexane +1 -butanol & 5.41 \\
\hline 1,4-Dioxane + cyclohexane +2 -butanol & 4.18 \\
\hline 1,4-Dioxane + cyclohexane + 2-methyl-1-propanol & 4.01 \\
\hline 1,4-Dioxane + 2,2,4-trimethylpentane + 1-propanol & 3.51 \\
\hline 1,4-Dioxane + 2,2,4-trimethylpentane +2 -propanol & 4.89 \\
\hline 1,4-Dioxane + 2,2,4-trimethylpentane + 1-butanol & 4.82 \\
\hline 1,4-Dioxane $+2,2,4$-trimethylpentane +2 -butanol & 4.32 \\
\hline 1,4-Dioxane $+2,2,4$-trimethylpentane +2 -methyl-1-propanol & 2.54 \\
\hline
\end{tabular}

${ }^{\mathrm{a}} \operatorname{Dev}(\%)=(100 / N) \Sigma\left|\left[x_{1}{ }^{\mathrm{exp}}-x_{1}{ }^{\mathrm{cal}}\right] / x_{1}{ }^{\mathrm{exp}}\right|$.

\subsection{Anthracene solubility data in ternary alkane + $1,1^{\prime}$-oxybisbutane + alcohol solvent mixtures}

\section{Components:}

(1) Anthracene; $\mathrm{C}_{14} \mathrm{H}_{10}$; [120-12-7]

(2) Heptane; $\mathrm{C}_{7} \mathrm{H}_{16} ;[142-82-5]$

(3) 1,1'-Oxybisbutane; $\mathrm{C}_{5} \mathrm{H}_{12} \mathrm{O}$; [142-96-1]

(4) 1-Propanol; $\mathrm{C}_{3} \mathrm{H}_{8} \mathrm{O} ;$; $\left.71-23-8\right]$

\begin{tabular}{ll}
\hline Variables: & Prepared by: \\
$T / \mathrm{K}=298.15 ;$ Solvent Composition & W. E. Acree, Jr.
\end{tabular}

Experimental Values

Solubility of anthracene in heptane $+1,1^{\prime}$-oxybisbutane +1 -propanol mixtures $^{\mathrm{a}}$

\begin{tabular}{|c|c|c|c|c|}
\hline$x_{3}{ }^{(\mathrm{s})}$ & $x_{4}{ }^{(\mathrm{s})}$ & $x_{3}$ & $x_{4}$ & $x_{1}$ \\
\hline 0.0000 & 0.0000 & 0.0000 & 0.0000 & 0.001571 \\
\hline 1.0000 & 0.0000 & 0.9964 & 0.0000 & 0.003615 \\
\hline 0.0000 & 1.0000 & 0.0000 & 0.9994 & 0.000591 \\
\hline 0.2156 & 0.4883 & 0.2152 & 0.4874 & 0.001771 \\
\hline 0.0833 & 0.8087 & 0.0832 & 0.8079 & 0.001042 \\
\hline 0.1736 & 0.3808 & 0.1733 & 0.3801 & 0.001777 \\
\hline 0.1508 & 0.6450 & 0.1506 & 0.6441 & 0.001441 \\
\hline 0.5668 & 0.2090 & 0.5652 & 0.2084 & 0.002802 \\
\hline 0.5307 & 0.3533 & 0.5293 & 0.3523 & 0.002694 \\
\hline 0.1062 & 0.8154 & 0.1061 & 0.8145 & 0.001073 \\
\hline 0.0582 & 0.6332 & 0.0581 & 0.6324 & 0.001247 \\
\hline 0.2409 & 0.6702 & 0.2405 & 0.6691 & 0.001635 \\
\hline 0.3160 & 0.5829 & 0.3154 & 0.5818 & 0.001957 \\
\hline 0.1106 & 0.2440 & 0.1104 & 0.2436 & 0.001706 \\
\hline 0.5496 & 0.2765 & 0.5481 & 0.2757 & 0.002789 \\
\hline 0.3491 & 0.4060 & 0.3483 & 0.4051 & 0.002158 \\
\hline 0.0728 & 0.3039 & 0.0727 & 0.3034 & 0.001604 \\
\hline 0.0598 & 0.7858 & 0.0597 & 0.7850 & 0.001030 \\
\hline 0.1475 & 0.1787 & 0.1472 & 0.1784 & 0.001796 \\
\hline 0.0682 & 0.5334 & 0.0681 & 0.5327 & 0.001382 \\
\hline 0.2989 & 0.1932 & 0.2983 & 0.1928 & 0.002128 \\
\hline 0.3801 & 0.1870 & 0.3793 & 0.1866 & 0.002130 \\
\hline
\end{tabular}

${ }^{\mathrm{a}} x_{3}{ }^{(\mathrm{s})}$ : initial mole fraction of component 3 in the ternary solvent mixture, $x_{4}{ }^{(\mathrm{s})}$ : initial mole fraction of component 4 in the ternary solvent mixture; $x_{1}$ : mole fraction solubility of the solute; $x_{3}$ : mole fraction of component 3 in the quaternary solution; $x_{4}$ : mole fraction of component 4 in the quaternary solution.

\section{Auxiliary Information}

Method/Apparatus/Procedure:

Constant-temperature bath, calorimetric thermometer, and an ultraviolet/ visible spectrophotometer.

Ternary solvent mixtures were prepared by mass. Excess solute and solvent were placed in amber glass bottles and allowed to equilibrate for several days at constant temperature. Attainment of equilibrium was verified by several repetitive measurements and by approaching equilibrium from supersaturation. Aliquots of saturated solutions were transferred through a coarse filter into volumetric flasks, weighed and diluted with methanol. Molar concentrations were determined by spectrophotometric measurements at $356 \mathrm{~nm}$. 
Source and Purity of Chemicals:

(1) $99+\%$, Acros Chemical Company, USA, was recrystallized three times from acetone.

(2) $99+\%$, HPLC grade, Aldrich Chemical Company, Milwaukee, WI, USA (3) $99.3 \%$, anhydrous, Aldrich Chemical Company.

(4) $99+\%$, anhydrous, Aldrich Chemical Company.

Components $2-4$ were stored over molecular sieves and distilled shortly before use.

\section{Estimated Error:}

Temperature: $\pm 0.1 \mathrm{~K}$

$x_{3}{ }^{(\mathrm{s})}: \pm 0.0001$.

$x_{4}{ }^{(\mathrm{s})}: \pm 0.0001$.

$x_{1}: \pm 1.5 \%$ (relative error).

\section{Components:}

(1) Anthracene; $\mathrm{C}_{14} \mathrm{H}_{10} ;[120-12-7]$

(2) Heptane; $\mathrm{C}_{7} \mathrm{H}_{16} ;[142-82-5]$

(3) 1,1'-Oxybisbutane; $\mathrm{C}_{8} \mathrm{H}_{18} \mathrm{O}$; [142-96-1]

(4) 2-Propanol; $\mathrm{C}_{3} \mathrm{H}_{8} \mathrm{O} ;[67-63-0]$

\begin{tabular}{ll}
\hline Variables: & Prepared by: \\
$T / \mathrm{K}=298.15 ;$ Solvent Composition & W. E. Acree, Jr. \\
\hline
\end{tabular}

\section{Experimental Values}

Solubility of anthracene in heptane $+1,1^{\prime}$-oxybisbutane +2 -propanol mixtures $^{\mathrm{a}}$

\begin{tabular}{|c|c|c|c|c|}
\hline$x_{3}{ }^{(\mathrm{s})}$ & $x_{4}{ }^{(\mathrm{s})}$ & $x_{3}$ & $x_{4}$ & $x_{1}$ \\
\hline 0.0000 & 0.0000 & 0.0000 & 0.0000 & 0.001571 \\
\hline 1.0000 & 0.0000 & 0.9964 & 0.0000 & 0.003615 \\
\hline 0.0000 & 1.0000 & 0.0000 & 0.9996 & 0.000411 \\
\hline 0.2275 & 0.5065 & 0.2271 & 0.5056 & 0.001686 \\
\hline 0.0843 & 0.8172 & 0.0842 & 0.8165 & 0.000873 \\
\hline 0.1837 & 0.4095 & 0.1834 & 0.4088 & 0.001705 \\
\hline 0.1528 & 0.6687 & 0.1526 & 0.6678 & 0.001280 \\
\hline 0.5867 & 0.2124 & 0.5850 & 0.2118 & 0.002844 \\
\hline 0.5445 & 0.3604 & 0.5440 & 0.3601 & 0.002651 \\
\hline 0.1119 & 0.8176 & 0.1118 & 0.8168 & 0.000927 \\
\hline 0.0664 & 0.6514 & 0.0663 & 0.6507 & 0.001119 \\
\hline 0.2460 & 0.6726 & 0.2456 & 0.6716 & 0.001467 \\
\hline 0.3283 & 0.5865 & 0.3277 & 0.5854 & 0.001801 \\
\hline 0.1244 & 0.2662 & 0.1242 & 0.2658 & 0.001679 \\
\hline 0.5708 & 0.2795 & 0.5692 & 0.2787 & 0.002763 \\
\hline 0.3601 & 0.4187 & 0.3593 & 0.4178 & 0.002126 \\
\hline 0.0802 & 0.3299 & 0.0801 & 0.3294 & 0.001554 \\
\hline 0.0585 & 0.8147 & 0.0584 & 0.8132 & 0.000829 \\
\hline 0.1689 & 0.1899 & 0.1686 & 0.1896 & 0.001821 \\
\hline 0.0735 & 0.5603 & 0.0734 & 0.5596 & 0.001256 \\
\hline 0.3320 & 0.1972 & 0.3313 & 0.1968 & 0.002228 \\
\hline 0.4149 & 0.2018 & 0.4139 & 0.2013 & 0.002380 \\
\hline \multicolumn{5}{|c|}{ 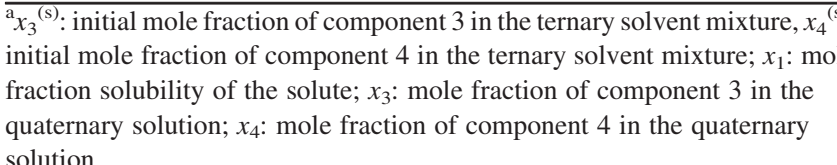 } \\
\hline
\end{tabular}

\section{Auxiliary Information}

\section{Method/Apparatus/Procedure:}

Constant-temperature bath, calorimetric thermometer, and an ultraviolet/ visible spectrophotometer.
Ternary solvent mixtures were prepared by mass. Excess solute and solvent were placed in amber glass bottles and allowed to equilibrate for several days at constant temperature. Attainment of equilibrium was verified by several repetitive measurements and by approaching equilibrium from supersaturation. Aliquots of saturated solutions were transferred through a coarse filter into volumetric flasks, weighed and diluted with methanol. Molar concentrations were determined by spectrophotometric measurements at $356 \mathrm{~nm}$.

\section{Source and Purity of Chemicals:}

(1) $99+\%$, Acros Chemical Company, USA, was recrystallized three times from acetone.

(2) $99+\%$, HPLC grade, Aldrich Chemical Company, Milwaukee, WI, USA. (3) $99.3 \%$, anhydrous, Aldrich Chemical Company.

(4) $99+\%$, anhydrous, Aldrich Chemical Company.

Components 2-4 were stored over molecular sieves and distilled shortly before use.

\section{Estimated Error:}

Temperature: $\pm 0.1 \mathrm{~K}$.

$x_{3}{ }^{(s)}: \pm 0.0001$.

$x_{4}{ }^{(\mathrm{s})}: \pm 0.0001$.

$x_{1}: \pm 1.5 \%$ (relative error)

\begin{tabular}{ll}
\hline \hline Components: & Original Measurements: \\
(1) Anthracene; $\mathrm{C}_{14} \mathrm{H}_{10} ;[120-12-7]$ & ${ }^{20} \mathrm{~K}$. J. Pribyla and W. E. \\
(2) Heptane; $\mathrm{C}_{7} \mathrm{H}_{16} ;[142-82-5]$ & Acree, Jr., J. Chem. Eng. \\
(3) $1,1^{\prime}-$-Oxybisbutane; $\mathrm{C}_{8} \mathrm{H}_{18} \mathrm{O} ;[142-96-1]$ & Data 44, 1259 (1999). \\
(4) 1-Butanol; $\mathrm{C}_{4} \mathrm{H}_{10} \mathrm{O} ;[71-36-3]$ & \\
\hline Variables: & Prepared by: \\
$T / \mathrm{K}=298.15 ;$ Solvent Composition & W. E. Acree, Jr. \\
\hline
\end{tabular}

Experimental Values

Solubility of anthracene in heptane $+1,1^{\prime}$-oxybisbutane +1 -butanol mixtures ${ }^{\mathrm{a}}$

\begin{tabular}{lcccc}
\hline \hline$x_{3}{ }^{(\mathrm{s})}$ & $x_{4}{ }^{(\mathrm{s})}$ & $x_{3}$ & $x_{4}$ & $x_{1}$ \\
\hline 0.0000 & 0.0000 & 0.0000 & 0.0000 & 0.001571 \\
1.0000 & 0.0000 & 0.9964 & 0.0000 & 0.003615 \\
0.0000 & 1.0000 & 0.0000 & 0.9992 & 0.000801 \\
0.2513 & 0.4580 & 0.2508 & 0.4751 & 0.001993 \\
0.0964 & 0.7913 & 0.0963 & 0.7903 & 0.001248 \\
0.1983 & 0.3638 & 0.1979 & 0.3631 & 0.001895 \\
0.1682 & 0.6280 & 0.1679 & 0.6270 & 0.001664 \\
0.6137 & 0.1748 & 0.6118 & 0.1743 & 0.003024 \\
0.5788 & 0.3198 & 0.5771 & 0.5189 & 0.002861 \\
0.1250 & 0.7955 & 0.1248 & 0.7944 & 0.001330 \\
0.0757 & 0.6158 & 0.0755 & 0.6149 & 0.001447 \\
0.2762 & 0.6356 & 0.2757 & 0.6344 & 0.001916 \\
0.3584 & 0.5467 & 0.3576 & 0.5455 & 0.002223 \\
0.1297 & 0.2351 & 0.1295 & 0.2347 & 0.001796 \\
0.5962 & 0.2489 & 0.5909 & 0.2482 & 0.002910 \\
0.3900 & 0.3754 & 0.3891 & 0.3745 & 0.002425 \\
0.0808 & 0.2986 & 0.0807 & 0.2981 & 0.001703 \\
0.0656 & 0.7851 & 0.0655 & 0.7842 & 0.001209 \\
0.1690 & 0.1717 & 0.1687 & 0.1714 & 0.001931 \\
0.0802 & 0.5185 & 0.0801 & 0.5177 & 0.001556 \\
0.3376 & 0.1781 & 0.3368 & 0.1771 & 0.002338 \\
0.4234 & 0.1801 & 0.4223 & 0.1796 & 0.002544 \\
\hline${ }^{a} x_{3}{ }^{(s)}:$ initial mole fraction of component 3 in the ternary solvent mixture, $x_{4}{ }^{(s)}$ : \\
initial mole fraction of component 4 in the ternary solvent mixture; $x_{1}:$ mole \\
fraction solubility of the solute; $x_{3}:$ mole fraction of component 3 in the \\
quaternary solution; $x_{4}:$ mole fraction of component 4 in the quaternary \\
solution. & & & & \\
& & & &
\end{tabular}




\section{Auxiliary Information}

Method/Apparatus/Procedure:

Constant-temperature bath, calorimetric thermometer, and an ultraviolet/ visible spectrophotometer.

Ternary solvent mixtures were prepared by mass. Excess solute and solvent were placed in amber glass bottles and allowed to equilibrate for several days at constant temperature. Attainment of equilibrium was verified by several repetitive measurements and by approaching equilibrium from supersaturation. Aliquots of saturated solutions were transferred through a coarse filter into volumetric flasks, weighed and diluted with methanol. Molar concentrations were determined by spectrophotometric measurements at $356 \mathrm{~nm}$.

\section{Source and Purity of Chemicals:}

(1) $99+\%$, Acros Chemical Company, USA, was recrystallized three times from acetone.

(2) $99+\%$, HPLC grade, Aldrich Chemical Company, Milwaukee, WI, USA

(3) $99.3 \%$, anhydrous, Aldrich Chemical Company.

(4) $99.8+\%$, HPLC grade, Aldrich Chemical Company.

Components 2-4 were stored over molecular sieves and distilled shortly before use.

\section{Estimated Error:}

Temperature: $\pm 0.1 \mathrm{~K}$.

$x_{3}{ }^{(\mathrm{s})}: \pm 0.0001$.

$x_{4}{ }^{(\mathrm{s})}: \pm 0.0001$.

$x_{1}: \pm 1.5 \%$ (relative error).

\section{Components:}

(1) Anthracene; $\mathrm{C}_{14} \mathrm{H}_{10} ;[120-12-7]$

(2) Heptane; $\mathrm{C}_{7} \mathrm{H}_{16} ;[142-82-5]$

(3) 1,1'-Oxybisbutane; $\mathrm{C}_{8} \mathrm{H}_{18} \mathrm{O}$; [142-96-1]

(4) 2-Butanol; $\mathrm{C}_{4} \mathrm{H}_{10} \mathrm{O}$; [78-92-2]

\begin{tabular}{ll}
\hline Variables: & Prepared by: \\
$T / \mathrm{K}=298.15 ;$ Solvent Composition & W. E. Acree, Jr. \\
\hline
\end{tabular}

Experimental Values

Solubility of anthracene in heptane $+1,1^{\prime}$-oxybisbutane +2 -butanol mixtures ${ }^{\mathrm{a}}$

\begin{tabular}{lcccc}
\hline \hline$x_{3}{ }^{(\mathrm{s})}$ & $x_{4}{ }^{(\mathrm{s})}$ & $x_{3}$ & $x_{4}$ & $x_{1}$ \\
\hline 0.0000 & 0.0000 & 0.0000 & 0.0000 & 0.001571 \\
1.0000 & 0.0000 & 0.9964 & 0.0000 & 0.003615 \\
0.0000 & 1.0000 & 0.0000 & 0.9994 & 0.000585 \\
0.2524 & 0.4595 & 0.2519 & 0.4586 & 0.001880 \\
0.1268 & 0.7278 & 0.1266 & 0.7269 & 0.001278 \\
0.1964 & 0.3626 & 0.1960 & 0.3619 & 0.001866 \\
0.1781 & 0.6210 & 0.1778 & 0.6200 & 0.001542 \\
0.6113 & 0.1772 & 0.6095 & 0.1267 & 0.002909 \\
0.5762 & 0.3165 & 0.5746 & 0.3156 & 0.002762 \\
0.1269 & 0.7901 & 0.1268 & 0.7892 & 0.001171 \\
0.0746 & 0.6175 & 0.0745 & 0.6167 & 0.001308 \\
0.2759 & 0.6369 & 0.2754 & 0.6358 & 0.001726 \\
0.3640 & 0.5399 & 0.3633 & 0.5388 & 0.002047 \\
0.1280 & 0.2295 & 0.1278 & 0.2291 & 0.001772 \\
0.5913 & 0.2523 & 0.5896 & 0.2516 & 0.002858
\end{tabular}

\begin{tabular}{|c|c|c|c|c|}
\hline 0.3941 & 0.3715 & 0.3932 & 0.3706 & 0.002319 \\
\hline 0.0826 & 0.2921 & 0.0825 & 0.2916 & 0.001659 \\
\hline 0.0652 & 0.7881 & 0.0651 & 0.7873 & 0.001060 \\
\hline 0.1714 & 0.1637 & 0.1711 & 0.1634 & 0.001892 \\
\hline 0.0784 & 0.5205 & 0.0783 & 0.5197 & 0.001460 \\
\hline 0.3387 & 0.1759 & 0.3379 & 0.1755 & 0.002233 \\
\hline 0.4265 & 0.1711 & 0.4255 & 0.1707 & 0.002420 \\
\hline
\end{tabular}

Auxiliary Information

\section{Method/Apparatus/Procedure:}

Constant-temperature bath, calorimetric thermometer, and an ultraviolet/ visible spectrophotometer.

Ternary solvent mixtures were prepared by mass. Excess solute and solvent were placed in amber glass bottles and allowed to equilibrate for several days at constant temperature. Attainment of equilibrium was verified by several repetitive measurements and by approaching equilibrium from supersaturation. Aliquots of saturated solutions were transferred through a coarse filter into volumetric flasks, weighed and diluted with methanol. Molar concentrations were determined by spectrophotometric measurements at $356 \mathrm{~nm}$.

\section{Source and Purity of Chemicals:}

(1) $99+\%$, Acros Chemical Company, USA, was recrystallized three times from acetone.

(2) $99+\%$, HPLC grade, Aldrich Chemical Company, Milwaukee, WI, USA.

(3) $99.3 \%$, anhydrous, Aldrich Chemical Company.

(4) $99+\%$, anhydrous, Aldrich Chemical Company.

Components 2-4 were stored over molecular sieves and distilled shortly before use.

\section{Estimated Error:}

Temperature: $\pm 0.1 \mathrm{~K}$.

$x_{3}{ }^{(\mathrm{s})}: \pm 0.0001$.

$x_{4}{ }^{(\mathrm{s})}: \pm 0.0001$.

$x_{1}: \pm 1.5 \%$ (relative error).

\begin{tabular}{|c|c|}
\hline $\begin{array}{l}\text { Components: } \\
\text { (1) Anthracene; } \mathrm{C}_{14} \mathrm{H}_{10} ;[120-12-7] \\
\text { (2) Heptane; } \mathrm{C}_{7} \mathrm{H}_{16} ;[142-82-5] \\
\text { (3) 1,1'-Oxybisbutane; } \mathrm{C}_{8} \mathrm{H}_{18} \mathrm{O} ;[142-96-1] \\
\text { (4) 2-Methyl-1-propanol; } \mathrm{C}_{4} \mathrm{H}_{10} \mathrm{O} ;[78-83-1]\end{array}$ & $\begin{array}{l}\text { Original Measurements: } \\
{ }^{20} \text { K. J. Pribyla and W. E. } \\
\text { Acree, Jr., J. Chem. Eng. } \\
\text { Data 44, } 1259 \text { (1999). }\end{array}$ \\
\hline $\begin{array}{l}\text { Variables: } \\
T / \mathrm{K}=298.15 \text {; Solvent Composition }\end{array}$ & $\begin{array}{l}\text { Prepared by: } \\
\text { W. E. Acree, Jr. }\end{array}$ \\
\hline
\end{tabular}




\section{Experimental Values}

Solubility of anthracene in heptane $+1,1^{\prime}$-oxybisbutane +2 -methyl-1-propanol mixtures ${ }^{\mathrm{a}}$

\begin{tabular}{|c|c|c|c|c|}
\hline$x_{3}{ }^{(\mathrm{s})}$ & $x_{4}^{(\mathrm{s})}$ & $x_{3}$ & $x_{4}$ & $x_{1}$ \\
\hline 0.0000 & 0.0000 & 0.0000 & 0.0000 & 0.001571 \\
\hline 1.0000 & 0.0000 & 0.9964 & 0.0000 & 0.003615 \\
\hline 0.0000 & 1.0000 & 0.0000 & 0.9995 & 0.000470 \\
\hline 0.2541 & 0.4532 & 0.2536 & 0.4524 & 0.001815 \\
\hline 0.0958 & 0.7912 & 0.0957 & 0.7904 & 0.000968 \\
\hline 0.1957 & 0.3630 & 0.1954 & 0.3624 & 0.001784 \\
\hline 0.1773 & 0.6224 & 0.1771 & 0.6215 & 0.001382 \\
\hline 0.6149 & 0.1748 & 0.6131 & 0.1743 & 0.002910 \\
\hline 0.5803 & 0.3132 & 0.5787 & 0.3123 & 0.002725 \\
\hline 0.1278 & 0.7938 & 0.1277 & 0.7930 & 0.001026 \\
\hline 0.0753 & 0.6137 & 0.0752 & 0.6130 & 0.001175 \\
\hline 0.2807 & 0.6333 & 0.2802 & 0.6323 & 0.001616 \\
\hline 0.3619 & 0.5407 & 0.3612 & 0.5396 & 0.002003 \\
\hline 0.1286 & 0.2319 & 0.1284 & 0.2315 & 0.001752 \\
\hline 0.5963 & 0.2453 & 0.5946 & 0.2446 & 0.002832 \\
\hline 0.3924 & 0.3733 & 0.3915 & 0.3725 & 0.002213 \\
\hline 0.0833 & 0.2919 & 0.0832 & 0.2914 & 0.001562 \\
\hline 0.0679 & 0.7838 & 0.0678 & 0.7831 & 0.000917 \\
\hline 0.1731 & 0.1666 & 0.1728 & 0.1663 & 0.001888 \\
\hline 0.0798 & 0.5198 & 0.0797 & 0.5191 & 0.001310 \\
\hline 0.3384 & 0.1793 & 0.3376 & 0.1789 & 0.002223 \\
\hline 0.4235 & 0.1839 & 0.4225 & 0.1834 & 0.002461 \\
\hline
\end{tabular}

${ }^{\mathrm{a}} x_{3}{ }^{(\mathrm{s})}$ : initial mole fraction of component 3 in the ternary solvent mixture, $x_{4}{ }^{(\mathrm{s})}$ initial mole fraction of component 4 in the ternary solvent mixture; $x_{1}$ : mole fraction solubility of the solute; $x_{3}$ : mole fraction of component 3 in the quaternary solution; $x_{4}$ : mole fraction of component 4 in the quaternary solution.

\section{Auxiliary Information}

\section{Method/Apparatus/Procedure:}

Constant-temperature bath, calorimetric thermometer, and an ultraviolet/ visible spectrophotometer.

Ternary solvent mixtures were prepared by mass. Excess solute and solvent were placed in amber glass bottles and allowed to equilibrate for several days at constant temperature. Attainment of equilibrium was verified by several repetitive measurements and by approaching equilibrium from supersaturation. Aliquots of saturated solutions were transferred through a coarse filter into volumetric flasks, weighed and diluted with methanol. Molar concentrations were determined by spectrophotometric measurements at $356 \mathrm{~nm}$.

\section{Source and Purity of Chemicals:}

(1) $99+\%$, Acros Chemical Company, USA, was recrystallized three times from acetone.

(2) $99+\%$, HPLC grade, Aldrich Chemical Company, Milwaukee, WI, USA. (3) $99.3 \%$, anhydrous, Aldrich Chemical Company.

(4) $99+\%$, anhydrous, Aldrich Chemical Company.

Components 2-4 were stored over molecular sieves and distilled shortly before use.

\section{Estimated Error:}

Temperature: $\pm 0.1 \mathrm{~K}$

$x_{3}^{(\mathrm{s})}: \pm 0.0001$.

$x_{4}{ }^{(\mathrm{s})}: \pm 0.0001$

$x_{1}: \pm 1.5 \%$ (relative error).

\begin{tabular}{|c|c|}
\hline $\begin{array}{l}\text { Components: } \\
\text { (1) Anthracene; } \mathrm{C}_{14} \mathrm{H}_{10} ;[120-12-7] \\
\text { (2) Cyclohexane; } \mathrm{C}_{6} \mathrm{H}_{12} ;[110-82-7] \\
\text { (3) 1,1'-Oxybisbutane; } \mathrm{C}_{5} \mathrm{H}_{12} \mathrm{O} ; \\
\text { [142-96-1] } \\
\text { (4) 1-Propanol; } \mathrm{C}_{3} \mathrm{H}_{8} \mathrm{O} ;[71-23-8]\end{array}$ & $\begin{array}{l}\text { Original Measurements: } \\
{ }^{21} \text { K. J. Pribyla and W. E. Acree, } \\
\text { Jr., J. Chem. Eng. Data 44, } 1020 \\
(1999) \text {. }\end{array}$ \\
\hline $\begin{array}{l}\text { Variables: } \\
T / \mathrm{K}=298.15 \text {; Solvent Composition }\end{array}$ & $\begin{array}{l}\text { Prepared by: } \\
\text { W. E. Acree, Jr. }\end{array}$ \\
\hline
\end{tabular}

Experimental Values

Solubility of anthracene in cyclohexane $+1,1^{\prime}$-oxybisbutane +1 -propanol mixtures $^{\mathrm{a}}$

\begin{tabular}{lcccc}
\hline \hline$x_{3}^{(\mathrm{s})}$ & $x_{4}{ }^{(\mathrm{s})}$ & $x_{3}$ & $x_{4}$ & $x_{1}$ \\
\hline 0.0000 & 0.0000 & 0.0000 & 0.0000 & 0.001553 \\
1.0000 & 0.0000 & 0.9964 & 0.0000 & 0.003615 \\
0.0000 & 1.0000 & 0.0000 & 0.9994 & 0.000591 \\
0.2048 & 0.4725 & 0.2044 & 0.4716 & 0.001883 \\
0.0829 & 0.7919 & 0.0828 & 0.7910 & 0.001078 \\
0.1571 & 0.3599 & 0.1568 & 0.3592 & 0.001868 \\
0.1444 & 0.6322 & 0.1442 & 0.6313 & 0.001477 \\
0.5557 & 0.1911 & 0.5541 & 0.1906 & 0.002866 \\
0.5146 & 0.3534 & 0.5132 & 0.3524 & 0.002707 \\
0.1064 & 0.8015 & 0.1063 & 0.8006 & 0.001114 \\
0.0611 & 0.6006 & 0.0610 & 0.5998 & 0.001285 \\
0.2761 & 0.6272 & 0.2756 & 0.6261 & 0.001783 \\
0.3104 & 0.5792 & 0.3098 & 0.5780 & 0.002007 \\
0.1013 & 0.2196 & 0.1011 & 0.2192 & 0.001817 \\
0.5267 & 0.2862 & 0.5352 & 0.2854 & 0.002812 \\
0.3307 & 0.4042 & 0.3300 & 0.4033 & 0.002260 \\
0.0673 & 0.2755 & 0.0672 & 0.2750 & 0.001700 \\
0.0581 & 0.7822 & 0.0580 & 0.7814 & 0.001021 \\
0.1353 & 0.1581 & 0.1350 & 0.1578 & 0.001946 \\
0.0636 & 0.5051 & 0.0635 & 0.5044 & 0.001432 \\
0.2819 & 0.1716 & 0.2813 & 0.1712 & 0.002258 \\
0.3002 & 0.1940 & 0.2995 & 0.1935 & 0.002409 \\
\hline
\end{tabular}

${ }^{\mathrm{a}} x_{3}{ }^{(\mathrm{s})}$ : initial mole fraction of component 3 in the ternary solvent mixture, $x_{4}{ }^{(\mathrm{s})}$ : initial mole fraction of component 4 in the ternary solvent mixture; $x_{1}$ : mole fraction solubility of the solute; $x_{3}$ : mole fraction of component 3 in the quaternary solution; $x_{4}$ : mole fraction of component 4 in the quaternary solution.

\section{Auxiliary Information}

\section{Method/Apparatus/Procedure:}

Constant-temperature bath, calorimetric thermometer, and an ultraviolet/ visible spectrophotometer.

Ternary solvent mixtures were prepared by mass. Excess solute and solvent were placed in amber glass bottles and allowed to equilibrate for several days at constant temperature. Attainment of equilibrium was verified by several repetitive measurements and by approaching equilibrium from supersaturation. Aliquots of saturated solutions were transferred through a coarse filter into volumetric flasks, weighed and diluted with methanol. Molar concentrations were determined by spectrophotometric measurements at $356 \mathrm{~nm}$.

Source and Purity of Chemicals:

(1) $99+\%$, Acros Chemical Company, USA, was recrystallized three times from acetone.

(2) $99+\%$, HPLC grade, Aldrich Chemical Company, Milwaukee, WI, USA.

(3) $99.3 \%$, anhydrous, Aldrich Chemical Company.

(4) $99+\%$, anhydrous, Aldrich Chemical Company.

Components 2-4 were stored over molecular sieves and distilled shortly before use. 
Estimated Error:

Temperature: $\pm 0.1 \mathrm{~K}$.

$x_{3}{ }^{(\mathrm{s})}: \pm 0.0001$.

$x_{4}{ }^{(\mathrm{s})}: \pm 0.0001$.

$x_{1}: \pm 1.5 \%$ (relative error).

\begin{tabular}{|c|c|}
\hline $\begin{array}{l}\text { Components: } \\
\text { (1) Anthracene; } \mathrm{C}_{14} \mathrm{H}_{10} ;[120-12-7] \\
\text { (2) Cyclohexane; } \mathrm{C}_{6} \mathrm{H}_{12} ;[110-82-7] \\
\text { (3) 1,1'-Oxybisbutane; } \mathrm{C}_{8} \mathrm{H}_{18} \mathrm{O} ; \\
\text { [142-96-1] } \\
\text { (4) 2-Propanol; } \mathrm{C}_{3} \mathrm{H}_{8} \mathrm{O} ;[67-63-0]\end{array}$ & $\begin{array}{l}\text { Original Measurements: } \\
{ }^{21} \text { K. J. Pribyla and W. E. Acree, } \\
\text { Jr., J. Chem. Eng. Data 44, } 1020 \\
\text { (1999). }\end{array}$ \\
\hline $\begin{array}{l}\text { Variables: } \\
T / \mathrm{K}=298.15 \text {; Solvent Composition }\end{array}$ & $\begin{array}{l}\text { Prepared by: } \\
\text { W. E. Acree, Jr. }\end{array}$ \\
\hline
\end{tabular}

Experimental Values

Solubility of anthracene in cyclohexane $+1,1^{\prime}$-oxybisbutane +2 -propanol mixtures $^{\mathrm{a}}$

\begin{tabular}{lcccc}
\hline \hline$x_{3}{ }^{(\mathrm{s})}$ & $x_{4}{ }^{(\mathrm{s})}$ & $x_{3}$ & $x_{4}$ & $x_{1}$ \\
\hline 0.0000 & 0.0000 & 0.0000 & 0.0000 & 0.001553 \\
1.0000 & 0.0000 & 0.9964 & 0.0000 & 0.003615 \\
0.0000 & 1.0000 & 0.0000 & 0.9996 & 0.000411 \\
0.2085 & 0.4629 & 0.2081 & 0.4621 & 0.001764 \\
0.0804 & 0.7946 & 0.0803 & 0.7939 & 0.000901 \\
0.1595 & 0.3583 & 0.1592 & 0.3576 & 0.001829 \\
0.1423 & 0.6291 & 0.1421 & 0.6283 & 0.001337 \\
0.5532 & 0.1930 & 0.5516 & 0.1924 & 0.002866 \\
0.5205 & 0.3386 & 0.5191 & 0.3377 & 0.002609 \\
0.1095 & 0.8006 & 0.1094 & 0.7998 & 0.000943 \\
0.0624 & 0.5968 & 0.0623 & 0.5961 & 0.001176 \\
0.2398 & 0.6594 & 0.2394 & 0.6584 & 0.001487 \\
0.3150 & 0.5723 & 0.3144 & 0.5713 & 0.001816 \\
0.0876 & 0.2459 & 0.0874 & 0.2455 & 0.001783 \\
0.5322 & 0.2793 & 0.5308 & 0.2785 & 0.002725 \\
0.3408 & 0.3834 & 0.3401 & 0.3826 & 0.002166 \\
0.0659 & 0.2768 & 0.0658 & 0.2763 & 0.001631 \\
0.0581 & 0.7789 & 0.0580 & 0.7782 & 0.000876 \\
0.1342 & 0.1578 & 0.1339 & 0.1575 & 0.001903 \\
0.0649 & 0.5031 & 0.0648 & 0.5024 & 0.001330 \\
0.2799 & 0.1784 & 0.2793 & 0.1780 & 0.002287 \\
0.3611 & 0.1831 & 0.3602 & 0.1827 & 0.002434 \\
\hline${ }^{a} x_{3}{ }^{\left({ }^{3}\right)}$ : initial mole fraction of component 3 in the ternary solvent mixture, $x_{4}{ }^{(\mathrm{s})}$ : \\
initial mole fraction of component 4 in the ternary solvent mixture; $x_{1}:$ mole \\
fraction solubility of the solute; $x_{3}:$ mole fraction of component 3 in the \\
quaternary solution; $x_{4}:$ mole fraction of component 4 in the quaternary \\
solution. & & & & \\
& & & &
\end{tabular}

\section{Auxiliary Information}

\section{Method/Apparatus/Procedure:}

Constant-temperature bath, calorimetric thermometer, and an ultraviolet/ visible spectrophotometer.

Ternary solvent mixtures were prepared by mass. Excess solute and solvent were placed in amber glass bottles and allowed to equilibrate for several days at constant temperature. Attainment of equilibrium was verified by several repetitive measurements and by approaching equilibrium from supersaturation. Aliquots of saturated solutions were transferred through a coarse filter into volumetric flasks, weighed and diluted with methanol. Molar concentrations were determined by spectrophotometric measurements at $356 \mathrm{~nm}$.

Source and Purity of Chemicals:

(1) $99+\%$, Acros Chemical Company, USA, was recrystallized three times from acetone.

(2) $99+\%$, HPLC grade, Aldrich Chemical Company, Milwaukee, WI, USA.

(3) $99.3 \%$, anhydrous, Aldrich Chemical Company.

(4) $99+\%$, anhydrous, Aldrich Chemical Company.

Components 2-4 were stored over molecular sieves and distilled shortly before use.

Estimated Error:

Temperature: $\pm 0.1 \mathrm{~K}$.

$x_{3}{ }^{(s)}: \pm 0.0001$.

$x_{4}{ }^{(\mathrm{s})}: \pm 0.0001$.

$x_{1}: \pm 1.5 \%$ (relative error).

\section{Components:}

(1) Anthracene; $\mathrm{C}_{14} \mathrm{H}_{10} ;$ [120-12-7]

(2) Cyclohexane; $\mathrm{C}_{6} \mathrm{H}_{12} ;[110-82-7]$

(3) $1,1^{\prime}$-Oxybisbutane; $\mathrm{C}_{8} \mathrm{H}_{18} \mathrm{O}$; [142-96-1]

(4) 1-Butanol; $\mathrm{C}_{4} \mathrm{H}_{10} \mathrm{O} ;[$ [71-36-3]

\begin{tabular}{ll}
\hline Variables: & Prepared by: \\
$T / \mathrm{K}=298.15 ;$ Solvent Composition & W. E. Acree, Jr. \\
\hline
\end{tabular}

Experimental Values

Solubility of anthracene in cyclohexane $+1,1^{\prime}$-oxybisbutane +1 -butanol mixtures $^{\mathrm{a}}$

\begin{tabular}{lcccc}
\hline \hline$x_{3}{ }^{(\mathrm{s})}$ & $x_{4}{ }^{(\mathrm{s})}$ & $x_{3}$ & $x_{4}$ & $x_{1}$ \\
\hline 0.0000 & 0.0000 & 0.0000 & 0.0000 & 0.001553 \\
1.0000 & 0.0000 & 0.9964 & 0.0000 & 0.003615 \\
0.0000 & 1.0000 & 0.0000 & 0.9992 & 0.000801 \\
0.2276 & 0.4225 & 0.2271 & 0.4216 & 0.002015 \\
0.0932 & 0.7567 & 0.0931 & 0.7557 & 0.001310 \\
0.1718 & 0.3132 & 0.1715 & 0.3126 & 0.001956 \\
0.1623 & 0.5790 & 0.1620 & 0.5780 & 0.001718 \\
0.5686 & 0.1685 & 0.5669 & 0.1680 & 0.002936 \\
0.5536 & 0.3029 & 0.5520 & 0.3020 & 0.002840 \\
0.1237 & 0.7723 & 0.1235 & 0.7712 & 0.001370 \\
0.0683 & 0.5553 & 0.0682 & 0.5545 & 0.001499 \\
0.2684 & 0.6125 & 0.2679 & 0.6113 & 0.001967 \\
0.3477 & 0.5235 & 0.3469 & 0.5223 & 0.002233 \\
0.1032 & 0.1886 & 0.1030 & 0.1882 & 0.001889 \\
0.5607 & 0.2398 & 0.5591 & 0.2391 & 0.002908 \\
0.3549 & 0.3531 & 0.3541 & 0.3523 & 0.002347 \\
0.0699 & 0.2412 & 0.0698 & 0.2408 & 0.001794 \\
0.0642 & 0.7467 & 0.0641 & 0.7458 & 0.001244 \\
0.1354 & 0.1357 & 0.1351 & 0.1354 & 0.001999 \\
0.0721 & 0.4500 & 0.0720 & 0.4493 & 0.001604 \\
0.2926 & 0.1472 & 0.2919 & 0.1469 & 0.002290 \\
0.3690 & 0.1587 & 0.3681 & 0.1583 & 0.002408 \\
\hline$x_{3}(\mathrm{~s})$ & & 0.351 &
\end{tabular}

${ }^{\mathrm{a}} x_{3}{ }^{(\mathrm{s})}$ : initial mole fraction of component 3 in the ternary solvent mixture, $x_{4}{ }^{(\mathrm{s})}$ : initial mole fraction of component 4 in the ternary solvent mixture; $x_{1}$ : mole fraction solubility of the solute; $x_{3}$ : mole fraction of component 3 in the quaternary solution; $x_{4}$ : mole fraction of component 4 in the quaternary solution. 


\section{Auxiliary Information}

\section{Method/Apparatus/Procedure:}

Constant-temperature bath, calorimetric thermometer, and an ultraviolet/ visible spectrophotometer.

Ternary solvent mixtures were prepared by mass. Excess solute and solvent were placed in amber glass bottles and allowed to equilibrate for several days at constant temperature. Attainment of equilibrium was verified by several repetitive measurements and by approaching equilibrium from supersaturation. Aliquots of saturated solutions were transferred through a coarse filter into volumetric flasks, weighed and diluted with methanol. Molar concentrations were determined by spectrophotometric measurements at $356 \mathrm{~nm}$.

\section{Source and Purity of Chemicals:}

(1) $99+\%$, Acros Chemical Company, USA, was recrystallized three times from acetone.

(2) $99+\%$, HPLC grade, Aldrich Chemical Company, Milwaukee, WI, USA

(3) $99.3 \%$, anhydrous, Aldrich Chemical Company.

(4) $99.8+\%$, HPLC grade, Aldrich Chemical Company.

Components 2-4 were stored over molecular sieves and distilled shortly before use.

\section{Estimated Error:}

Temperature: $\pm 0.1 \mathrm{~K}$

$x_{3}{ }^{(\mathrm{s})}: \pm 0.0001$.

$x_{4}{ }^{(\mathrm{s})}: \pm 0.0001$

$x_{1}: \pm 1.5 \%$ (relative error).

\section{Components:}

(1) Anthracene; $\mathrm{C}_{14} \mathrm{H}_{10} ;[120-12-7]$

(2) Cyclohexane; $\mathrm{C}_{6} \mathrm{H}_{12} ;[110-82-7]$

(3) 1,1'-Oxybisbutane; $\mathrm{C}_{8} \mathrm{H}_{18} \mathrm{O}$; [142-96-1]

(4) 2-Butanol; $\mathrm{C}_{4} \mathrm{H}_{10} \mathrm{O}$; [78-92-2]

\begin{tabular}{ll}
\hline Variables: & Prepared by: \\
$T / \mathrm{K}=298.15 ;$ Solvent Composition & W. E. Acree, Jr. \\
\hline
\end{tabular}

\section{Experimental Values}

Solubility of anthracene in cyclohexane $+1,1^{\prime}$-oxybisbutane +2 -butanol mixtures $^{\mathrm{a}}$

\begin{tabular}{lcccc}
\hline \hline$x_{3}{ }^{(\mathrm{s})}$ & $x_{4}{ }^{(\mathrm{s})}$ & $x_{3}$ & $x_{4}$ & $x_{1}$ \\
\hline 0.0000 & 0.0000 & 0.0000 & 0.0000 & 0.001553 \\
1.0000 & 0.0000 & 0.9964 & 0.0000 & 0.003615 \\
0.0000 & 1.0000 & 0.0000 & 0.9994 & 0.000585 \\
0.2263 & 0.4202 & 0.2259 & 0.4194 & 0.001953 \\
0.0883 & 0.7556 & 0.0882 & 0.7548 & 0.001118 \\
0.1620 & 0.3218 & 0.1617 & 0.3212 & 0.001915 \\
0.1639 & 0.5791 & 0.1636 & 0.5782 & 0.001594 \\
0.5718 & 0.1685 & 0.5701 & 0.1680 & 0.002910 \\
0.5549 & 0.3047 & 0.5534 & 0.3039 & 0.002764 \\
0.1227 & 0.7703 & 0.1226 & 0.7694 & 0.001184 \\
0.0692 & 0.5495 & 0.0691 & 0.5487 & 0.001399 \\
0.2677 & 0.6133 & 0.2672 & 0.6122 & 0.001779 \\
0.3530 & 0.5220 & 0.3523 & 0.5209 & 0.002112 \\
0.1068 & 0.1852 & 0.1066 & 0.1849 & 0.001888
\end{tabular}

$\begin{array}{lllll}0.5520 & 0.2327 & 0.5504 & 0.2320 & 0.002876 \\ 0.3553 & 0.3571 & 0.3545 & 0.3563 & 0.002334 \\ 0.0688 & 0.2384 & 0.0687 & 0.2380 & 0.001750 \\ 0.0623 & 0.7482 & 0.0622 & 0.7474 & 0.001074 \\ 0.1353 & 0.1363 & 0.1350 & 0.1360 & 0.001971 \\ 0.0646 & 0.4555 & 0.0645 & 0.4548 & 0.001513 \\ 0.2894 & 0.1478 & 0.2887 & 0.1475 & 0.002339 \\ 0.3818 & 0.1541 & 0.3808 & 0.1537 & 0.002541\end{array}$

${ }^{\mathrm{a}} x_{3}{ }^{(\mathrm{s})}$ : initial mole fraction of component 3 in the ternary solvent mixture, $x_{4}{ }^{(\mathrm{s})}$ : initial mole fraction of component 4 in the ternary solvent mixture; $x_{1}$ : mole fraction solubility of the solute; $x_{3}$ : mole fraction of component 3 in the quaternary solution; $x_{4}$ : mole fraction of component 4 in the quaternary solution.

\section{Auxiliary Information}

\section{Method/Apparatus/Procedure:}

Constant-temperature bath, calorimetric thermometer, and an ultraviolet/ visible spectrophotometer.

Ternary solvent mixtures were prepared by mass. Excess solute and solvent were placed in amber glass bottles and allowed to equilibrate for several days at constant temperature. Attainment of equilibrium was verified by several repetitive measurements and by approaching equilibrium from supersaturation. Aliquots of saturated solutions were transferred through a coarse filter into volumetric flasks, weighed and diluted with methanol. Molar concentrations were determined by spectrophotometric measurements at $356 \mathrm{~nm}$.

Source and Purity of Chemicals:

(1) $99+\%$, Acros Chemical Company, USA, was recrystallized three times from acetone.

(2) $99+\%$, HPLC grade, Aldrich Chemical Company, Milwaukee, WI, USA.

(3) $99.3 \%$, anhydrous, Aldrich Chemical Company.

(4) $99+\%$, anhydrous, Aldrich Chemical Company.

Components 2-4 were stored over molecular sieves and distilled shortly before use.

\section{Estimated Error:}

Temperature: $\pm 0.1 \mathrm{~K}$.

$x_{3}{ }^{(\mathrm{s})}: \pm 0.0001$.

$x_{4}{ }^{(s)}: \pm 0.0001$.

$x_{1}: \pm 1.5 \%$ (relative error).

\begin{tabular}{|c|c|}
\hline $\begin{array}{l}\text { Components: } \\
\text { (1) Anthracene; } \mathrm{C}_{14} \mathrm{H}_{10} ;[120-12-7] \\
\text { (2) Cyclohexane; } \mathrm{C}_{6} \mathrm{H}_{12} ;[110-82-7] \\
\text { (3) 1,1'-Oxybisbutane; } \mathrm{C}_{8} \mathrm{H}_{18} \mathrm{O} ; \\
\text { [142-96-1] } \\
\text { (4) 2-Methyl-1-propanol; } \mathrm{C}_{4} \mathrm{H}_{10} \mathrm{O} \text {; } \\
\text { [78-83-1] }\end{array}$ & $\begin{array}{l}\text { Original Measurements: } \\
{ }^{21} \text { K. J. Pribyla and W. E. Acree, } \\
\text { Jr., J. Chem. Eng. Data 44, } 1020 \\
(1999) \text {. }\end{array}$ \\
\hline $\begin{array}{l}\text { Variables: } \\
T / \mathrm{K}=298.15 ; \text { Solvent Composition }\end{array}$ & $\begin{array}{l}\text { Prepared by: } \\
\text { W. E. Acree, Jr. }\end{array}$ \\
\hline
\end{tabular}


Experimental Values

Solubility of anthracene in cyclohexane $+1,1^{\prime}$-oxybisbutane +2 -methyl-1propanol mixtures ${ }^{\mathrm{a}}$

\begin{tabular}{lcccc}
\hline \hline$x_{3}{ }^{(\mathrm{s})}$ & $x_{4}{ }^{(\mathrm{s})}$ & $x_{3}$ & $x_{4}$ & $x_{1}$ \\
\hline 0.0000 & 0.0000 & 0.0000 & 0.0000 & 0.001553 \\
1.0000 & 0.0000 & 0.9964 & 0.0000 & 0.003615 \\
0.0000 & 1.0000 & 0.0000 & 0.9995 & 0.000470 \\
0.2279 & 0.4132 & 0.2275 & 0.4124 & 0.001816 \\
0.0943 & 0.7510 & 0.0942 & 0.7503 & 0.000991 \\
0.1766 & 0.3052 & 0.1763 & 0.3046 & 0.001848 \\
0.1634 & 0.5744 & 0.1632 & 0.5736 & 0.001414 \\
0.5694 & 0.1686 & 0.5678 & 0.1681 & 0.002811 \\
0.5573 & 0.3059 & 0.5558 & 0.3051 & 0.002740 \\
0.1268 & 0.7694 & 0.1267 & 0.7686 & 0.001032 \\
0.0697 & 0.5503 & 0.0696 & 0.5496 & 0.001229 \\
0.2681 & 0.6128 & 0.2677 & 0.6118 & 0.001594 \\
0.3527 & 0.5251 & 0.3520 & 0.5241 & 0.001946 \\
0.1058 & 0.1875 & 0.1056 & 0.1872 & 0.001818 \\
0.5620 & 0.2305 & 0.5604 & 0.2299 & 0.002810 \\
0.3639 & 0.3433 & 0.3631 & 0.3425 & 0.002222 \\
0.0740 & 0.2402 & 0.0739 & 0.2398 & 0.001653 \\
0.0696 & 0.7413 & 0.0695 & 0.7406 & 0.000939 \\
0.1400 & 0.1377 & 0.1397 & 0.1374 & 0.001941 \\
0.0703 & 0.4527 & 0.0702 & 0.4521 & 0.001375 \\
0.2902 & 0.1523 & 0.2895 & 0.1520 & 0.002268 \\
0.3756 & 0.1556 & 0.3747 & 0.1552 & 0.002402 \\
\hline$x_{3}(5)$ & &
\end{tabular}

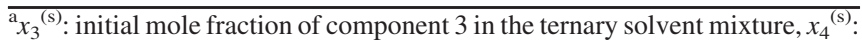
initial mole fraction of component 4 in the ternary solvent mixture; $x_{1}$ : mole fraction solubility of the solute; $x_{3}$ : mole fraction of component 3 in the quaternary solution; $x_{4}$ : mole fraction of component 4 in the quaternary solution.

\section{Auxiliary Information}

\section{Method/Apparatus/Procedure:}

Constant-temperature bath, calorimetric thermometer, and an ultraviolet/ visible spectrophotometer.

Ternary solvent mixtures were prepared by mass. Excess solute and solvent were placed in amber glass bottles and allowed to equilibrate for several days at constant temperature. Attainment of equilibrium was verified by several repetitive measurements and by approaching equilibrium from supersaturation. Aliquots of saturated solutions were transferred through a coarse filter into volumetric flasks, weighed and diluted with methanol. Molar concentrations were determined by spectrophotometric measurements at $356 \mathrm{~nm}$.

\section{Source and Purity of Chemicals:}

(1) $99+\%$, Acros Chemical Company, USA, was recrystallized three times from acetone.

(2) $99+\%$, HPLC grade, Aldrich Chemical Company, Milwaukee, WI, USA.

(3) $99.3 \%$, anhydrous, Aldrich Chemical Company.

(4) $99+\%$, anhydrous, Aldrich Chemical Company.

Components 2-4 were stored over molecular sieves and distilled shortly before use.

\section{Estimated Error:}

Temperature: $\pm 0.1 \mathrm{~K}$.

$x_{3}{ }^{(\mathrm{s})}: \pm 0.0001$.

$x_{4}{ }^{(\mathrm{s})}: \pm 0.0001$.

$x_{1}: \pm 1.5 \%$ (relative error).

\begin{tabular}{|c|c|}
\hline $\begin{array}{l}\text { Components: } \\
\text { (1) Anthracene; } \mathrm{C}_{14} \mathrm{H}_{10} ;[120-12-7] \\
\text { (2) } 2,2,4-\text { Trimethylpentane; } \mathrm{C}_{8} \mathrm{H}_{18} \text {; } \\
\text { [540-84-1] } \\
\text { (3) } 1,1^{\prime}-\text { Oxybisbutane; } \mathrm{C}_{5} \mathrm{H}_{12} \mathrm{O} \text {; } \\
\text { [142-96-1] } \\
\text { (4) 1-Propanol; } \mathrm{C}_{3} \mathrm{H}_{8} \mathrm{O} ;[71-23-8]\end{array}$ & $\begin{array}{l}\text { Original Measurements: } \\
{ }^{19} \text { K. J. Pribyla, M. A. Spurgin, I. } \\
\text { Chuca, and W. E. Acree, Jr., J. } \\
\text { Chem. Eng. Data 44, } 1265 \text { (1999). }\end{array}$ \\
\hline $\begin{array}{l}\text { Variables: } \\
T / \mathrm{K}=298.15 \text {; Solvent Composition } \\
\end{array}$ & $\begin{array}{l}\text { Prepared by: } \\
\text { W. E. Acree, Jr. }\end{array}$ \\
\hline
\end{tabular}

Experimental Values

Solubility of anthracene in 2,2,4-trimethylpentane $+1,1^{\prime}$-oxybisbutane +1 propanol mixtures $^{\mathrm{a}}$

\begin{tabular}{lcccc}
\hline \hline$x_{3}{ }^{(\mathrm{s})}$ & $x_{4}{ }^{(\mathrm{s})}$ & $x_{3}$ & $x_{4}$ & $x_{1}$ \\
\hline 0.0000 & 0.0000 & 0.0000 & 0.0000 & 0.001074 \\
1.0000 & 0.0000 & 0.9964 & 0.0000 & 0.003615 \\
0.0000 & 1.0000 & 0.0000 & 0.9994 & 0.000591 \\
0.2322 & 0.5275 & 0.2318 & 0.5266 & 0.001614 \\
0.0841 & 0.8301 & 0.0840 & 0.8293 & 0.000976 \\
0.1911 & 0.4260 & 0.1908 & 0.4253 & 0.001530 \\
0.1564 & 0.6849 & 0.1562 & 0.6841 & 0.001292 \\
0.6297 & 0.2036 & 0.6279 & 0.2030 & 0.002825 \\
0.5363 & 0.3822 & 0.5349 & 0.3812 & 0.002608 \\
0.0954 & 0.8520 & 0.0953 & 0.8512 & 0.000987 \\
0.0671 & 0.6827 & 0.0670 & 0.6820 & 0.001028 \\
0.2596 & 0.6760 & 0.2592 & 0.6749 & 0.001633 \\
0.3345 & 0.6094 & 0.3338 & 0.6082 & 0.001956 \\
0.1563 & 0.3499 & 0.1561 & 0.3494 & 0.001432 \\
0.5910 & 0.2741 & 0.5893 & 0.2733 & 0.002829 \\
0.3955 & 0.4020 & 0.3947 & 0.4011 & 0.002143 \\
0.0880 & 0.3581 & 0.0879 & 0.3576 & 0.001265 \\
0.0546 & 0.8429 & 0.0546 & 0.8422 & 0.000866 \\
0.1782 & 0.1955 & 0.1779 & 0.1952 & 0.001538 \\
0.0736 & 0.5961 & 0.0735 & 0.5954 & 0.001128 \\
0.3493 & 0.2152 & 0.3486 & 0.2148 & 0.001955 \\
0.4710 & 0.1778 & 0.4699 & 0.1774 & 0.002308 \\
\hline$x_{3} 5$ & & &
\end{tabular}

${ }^{\mathrm{a}} x_{3}{ }^{(\mathrm{s})}$ : initial mole fraction of component 3 in the ternary solvent mixture, $x_{4}{ }^{(\mathrm{s})}$ : initial mole fraction of component 4 in the ternary solvent mixture; $x_{1}$ : mole fraction solubility of the solute; $x_{3}$ : mole fraction of component 3 in the quaternary solution; $x_{4}$ : mole fraction of component 4 in the quaternary solution.

\section{Auxiliary Information}

Method/Apparatus/Procedure:

Constant-temperature bath, calorimetric thermometer, and an ultraviolet/ visible spectrophotometer.

Ternary solvent mixtures were prepared by mass. Excess solute and solvent were placed in amber glass bottles and allowed to equilibrate for several days at constant temperature. Attainment of equilibrium was verified by several repetitive measurements and by approaching equilibrium from supersaturation. Aliquots of saturated solutions were transferred through a coarse filter into volumetric flasks, weighed and diluted with methanol. Molar concentrations were determined by spectrophotometric measurements at $356 \mathrm{~nm}$.

Source and Purity of Chemicals:

(1) $99+\%$, Acros Chemical Company, USA, was recrystallized three times from acetone.

(2) $99+\%$, HPLC grade, Aldrich Chemical Company, Milwaukee, WI, USA.

(3) $99.3 \%$, anhydrous, Aldrich Chemical Company.

(4) $99+\%$, anhydrous, Aldrich Chemical Company.

Components 2-4 were stored over molecular sieves and distilled shortly before use. 
Estimated Error:

Temperature: $\pm 0.1 \mathrm{~K}$

$x_{3}{ }^{(\mathrm{s})}: \pm 0.0001$

$x_{4}{ }^{(\mathrm{s})}: \pm 0.0001$

$x_{1}: \pm 1.5 \%$ (relative error).

\begin{tabular}{ll}
\hline \hline Components: & Original Measurements: \\
(1) Anthracene; $\mathrm{C}_{14} \mathrm{H}_{10} ;[120-12-7]$ & ${ }^{19}$ K. J. Pribyla, M. A. Spurgin, I. \\
(2) 2,2,4-Trimethylpentane; $\mathrm{C}_{8} \mathrm{H}_{18} ;$ & Chuca, and W. E. Acree, Jr., J. \\
[540-84-1] & Chem. Eng. Data 44, 1265 (1999). \\
$\begin{array}{l}\text { (3) 1,1'-Oxybisbutane; } \mathrm{C}_{8} \mathrm{H}_{18} \mathrm{O} ; \\
{[142-96-1]}\end{array}$ & \\
(4) 2-Propanol; $\mathrm{C}_{3} \mathrm{H}_{8} \mathrm{O} ;$ [67-63-0] & \\
\hline Variables: & Prepared by: \\
$T / \mathrm{K}=298.15 ;$ Solvent Composition & W. E. Acree, Jr. \\
\hline
\end{tabular}

Experimental Values

Solubility of anthracene in 2,2,4-trimethylpentane $+1,1^{\prime}$-oxybisbutane +2 propanol mixtures ${ }^{\mathrm{a}}$

\begin{tabular}{|c|c|c|c|c|}
\hline$x_{3}{ }^{(\mathrm{s})}$ & $x_{4}{ }^{(\mathrm{s})}$ & $x_{3}$ & $x_{4}$ & $x_{1}$ \\
\hline 0.0000 & 0.0000 & 0.0000 & 0.0000 & 0.001074 \\
\hline 1.0000 & 0.0000 & 0.9964 & 0.0000 & 0.003615 \\
\hline 0.0000 & 1.0000 & 0.0000 & 0.9996 & 0.000411 \\
\hline 0.2336 & 0.5237 & 0.2333 & 0.5229 & 0.001496 \\
\hline 0.0834 & 0.8265 & 0.0833 & 0.8258 & 0.000808 \\
\hline 0.1926 & 0.4284 & 0.1923 & 0.4278 & 0.001416 \\
\hline 0.1589 & 0.6771 & 0.1587 & 0.6763 & 0.001143 \\
\hline 0.5998 & 0.2146 & 0.5982 & 0.2140 & 0.002666 \\
\hline 0.5476 & 0.3587 & 0.5462 & 0.3578 & 0.002542 \\
\hline 0.1471 & 0.7714 & 0.1469 & 0.7706 & 0.001030 \\
\hline 0.0684 & 0.6779 & 0.0683 & 0.6773 & 0.000905 \\
\hline 0.2492 & 0.6802 & 0.2488 & 0.6792 & 0.001431 \\
\hline 0.3314 & 0.5913 & 0.3308 & 0.5903 & 0.001763 \\
\hline 0.1293 & 0.2886 & 0.1291 & 0.2882 & 0.001319 \\
\hline 0.5654 & 0.2981 & 0.5639 & 0.2973 & 0.002577 \\
\hline 0.3732 & 0.4279 & 0.3725 & 0.4271 & 0.001915 \\
\hline 0.0885 & 0.3575 & 0.0884 & 0.3571 & 0.001173 \\
\hline 0.0585 & 0.8278 & 0.0585 & 0.8272 & 0.000742 \\
\hline 0.1770 & 0.2093 & 0.1767 & 0.2090 & 0.001424 \\
\hline 0.0747 & 0.5888 & 0.0746 & 0.5882 & 0.001014 \\
\hline 0.3485 & 0.2137 & 0.3479 & 0.2133 & 0.001865 \\
\hline 0.4303 & 0.2133 & 0.4294 & 0.2128 & 0.002153 \\
\hline
\end{tabular}

\section{Auxiliary Information}

\section{Method/Apparatus/Procedure:}

Constant-temperature bath, calorimetric thermometer, and an ultraviolet/ visible spectrophotometer.

Ternary solvent mixtures were prepared by mass. Excess solute and solvent were placed in amber glass bottles and allowed to equilibrate for several days at constant temperature. Attainment of equilibrium was verified by several repetitive measurements and by approaching equilibrium from supersaturation. Aliquots of saturated solutions were transferred through a coarse filter into volumetric flasks, weighed and diluted with methanol. Molar concentrations were determined by spectrophotometric measurements at $356 \mathrm{~nm}$.
Source and Purity of Chemicals:

(1) $99+\%$, Acros Chemical Company, USA, was recrystallized three times from acetone.

(2) $99+\%$, HPLC grade, Aldrich Chemical Company, Milwaukee, WI, USA.

(3) $99.3 \%$, anhydrous, Aldrich Chemical Company.

(4) $99+\%$, anhydrous, Aldrich Chemical Company.

Components 2-4 were stored over molecular sieves and distilled shortly before use.

\section{Estimated Error:}

Temperature: $\pm 0.1 \mathrm{~K}$.

$x_{3}{ }^{(s)}: \pm 0.0001$.

$x_{4}{ }^{(\mathrm{s})}: \pm 0.0001$.

$x_{1}: \pm 1.5 \%$ (relative error).

\begin{tabular}{|c|c|}
\hline $\begin{array}{l}\text { Components: } \\
\text { (1) Anthracene; } \mathrm{C}_{14} \mathrm{H}_{10} ;[120-12-7] \\
\text { (2) } 2,2,4-\text {-Trimethylpentane; } \mathrm{C}_{8} \mathrm{H}_{18} \text {; } \\
\text { [540-84-1] } \\
\text { (3) } 1,1^{\prime}-\text {-Oxybisbutane; } \mathrm{C}_{8} \mathrm{H}_{18} \mathrm{O} ; \\
\text { [142-96-1] } \\
\text { (4) } 1 \text {-Butanol; } \mathrm{C}_{4} \mathrm{H}_{10} \mathrm{O} ;[71-36-3]\end{array}$ & $\begin{array}{l}\text { Original Measurements: } \\
{ }^{19} \text { K. J. Pribyla, M. A. Spurgin, I. } \\
\text { Chuca, and W. E. Acree, Jr., J. } \\
\text { Chem. Eng. Data 44, } 1265 \text { (1999). }\end{array}$ \\
\hline $\begin{array}{l}\text { Variables: } \\
T / \mathrm{K}=298.15 ; \text { Solvent Composition }\end{array}$ & $\begin{array}{l}\text { Prepared by: } \\
\text { W. E. Acree, Jr. }\end{array}$ \\
\hline
\end{tabular}

Experimental Values

Solubility of anthracene in 2,2,4-trimethylpentane $+1,1^{\prime}$-oxybisbutane +1 butanol mixtures ${ }^{\mathrm{a}}$

\begin{tabular}{lcccc}
\hline \hline$x_{3}{ }^{(\mathrm{s})}$ & $x_{4}{ }^{(\mathrm{s})}$ & $x_{3}$ & $x_{4}$ & $x_{1}$ \\
\hline 0.0000 & 0.0000 & 0.0000 & 0.0000 & 0.001074 \\
1.0000 & 0.0000 & 0.9964 & 0.0000 & 0.003615 \\
0.0000 & 1.0000 & 0.0000 & 0.9992 & 0.000801 \\
0.2328 & 0.5360 & 0.2324 & 0.5351 & 0.001646 \\
0.0818 & 0.8368 & 0.0817 & 0.8359 & 0.001120 \\
0.1910 & 0.4336 & 0.1907 & 0.4329 & 0.001532 \\
0.1558 & 0.6862 & 0.1556 & 0.6852 & 0.001442 \\
0.6055 & 0.2124 & 0.6038 & 0.2118 & 0.002734 \\
0.5445 & 0.3634 & 0.5431 & 0.3624 & 0.002633 \\
0.1066 & 0.8334 & 0.1065 & 0.8324 & 0.001190 \\
0.0678 & 0.6881 & 0.0677 & 0.6873 & 0.001171 \\
0.2431 & 0.6884 & 0.2427 & 0.6872 & 0.001684 \\
0.3233 & 0.6021 & 0.3227 & 0.6009 & 0.001959 \\
0.1344 & 0.2940 & 0.1342 & 0.2936 & 0.001394 \\
0.5682 & 0.2998 & 0.5667 & 0.2990 & 0.002668 \\
0.3718 & 0.4350 & 0.3710 & 0.4341 & 0.002097 \\
0.0892 & 0.3637 & 0.0891 & 0.3632 & 0.001273 \\
0.0604 & 0.8286 & 0.0503 & 0.8277 & 0.001063 \\
0.1741 & 0.2157 & 0.1738 & 0.2154 & 0.001491 \\
0.0728 & 0.5952 & 0.0727 & 0.5945 & 0.001186 \\
0.3429 & 0.2238 & 0.3422 & 0.2234 & 0.001936 \\
0.4257 & 0.2215 & 0.4248 & 0.2210 & 0.002179 \\
\hline$x_{3}(\mathrm{~s})$ & &
\end{tabular}

${ }^{\mathrm{a}} x_{3}{ }^{(\mathrm{s})}$ : initial mole fraction of component 3 in the ternary solvent mixture, $x_{4}{ }^{(\mathrm{s})}$ : initial mole fraction of component 4 in the ternary solvent mixture; $x_{1}$ : mole fraction solubility of the solute; $x_{3}$ : mole fraction of component 3 in the quaternary solution; $x_{4}$ : mole fraction of component 4 in the quaternary solution. 


\section{Auxiliary Information}

Method/Apparatus/Procedure:

Constant-temperature bath, calorimetric thermometer, and an ultraviolet/ visible spectrophotometer.

Ternary solvent mixtures were prepared by mass. Excess solute and solvent were placed in amber glass bottles and allowed to equilibrate for several days at constant temperature. Attainment of equilibrium was verified by several repetitive measurements and by approaching equilibrium from supersaturation. Aliquots of saturated solutions were transferred through a coarse filter into volumetric flasks, weighed and diluted with methanol. Molar concentrations were determined by spectrophotometric measurements at $356 \mathrm{~nm}$.

\section{Source and Purity of Chemicals:}

(1) $99+\%$, Acros Chemical Company, USA, was recrystallized three times from acetone.

(2) $99+\%$, HPLC grade, Aldrich Chemical Company, Milwaukee, WI, USA

(3) $99.3 \%$, anhydrous, Aldrich Chemical Company.

(4) $99.8+\%$, HPLC grade, Aldrich Chemical Company.

Components 2-4 were stored over molecular sieves and distilled shortly before use.

\section{Estimated Error:}

Temperature: $\pm 0.1 \mathrm{~K}$.

$x_{3}{ }^{(\mathrm{s})}: \pm 0.0001$.

$x_{4}{ }^{(\mathrm{s})}: \pm 0.0001$.

$x_{1}: \pm 1.5 \%$ (relative error).

\section{Components:}

(1) Anthracene; $\mathrm{C}_{14} \mathrm{H}_{10}$; [120-12-7]

(2) 2,2,4-Trimethylpentane; $\mathrm{C}_{8} \mathrm{H}_{18}$;

[540-84-1]

(3) 1,1'-Oxybisbutane; $\mathrm{C}_{8} \mathrm{H}_{18} \mathrm{O}$;

[142-96-1]

(4) 2-Butanol; $\mathrm{C}_{4} \mathrm{H}_{10} \mathrm{O}$; [78-92-2]

\begin{tabular}{ll}
\hline Variables: & Prepared by: \\
$T / \mathrm{K}=298.15 ;$ Solvent Composition & W. E. Acree, Jr. \\
\hline
\end{tabular}

\section{Experimental Values}

Solubility of anthracene in 2,2,4-trimethylpentane $+1,1^{\prime}$-oxybisbutane +2 butanol mixtures ${ }^{\text {a }}$

\begin{tabular}{lcccc}
\hline \hline$x_{3}{ }^{(\mathrm{s})}$ & $x_{4}{ }^{(\mathrm{s})}$ & $x_{3}$ & $x_{4}$ & $x_{1}$ \\
\hline 0.0000 & 0.0000 & 0.0000 & 0.0000 & 0.001074 \\
1.0000 & 0.0000 & 0.9964 & 0.0000 & 0.003615 \\
0.0000 & 1.0000 & 0.0000 & 0.9994 & 0.000585 \\
0.2599 & 0.4757 & 0.2595 & 0.4749 & 0.001678 \\
0.1216 & 0.7154 & 0.1215 & 0.7146 & 0.001187 \\
0.2101 & 0.3818 & 0.2098 & 0.3812 & 0.001606 \\
0.1797 & 0.6393 & 0.1794 & 0.6384 & 0.001403 \\
0.6286 & 0.1793 & 0.6269 & 0.1788 & 0.002771 \\
0.5848 & 0.3226 & 0.5832 & 0.3217 & 0.002707 \\
0.1260 & 0.8067 & 0.1259 & 0.8058 & 0.001097 \\
0.0783 & 0.6384 & 0.0788 & 0.6377 & 0.001124 \\
0.2797 & 0.6428 & 0.2792 & 0.6417 & 0.001658 \\
0.3701 & 0.5489 & 0.3694 & 0.5478 & 0.001989 \\
0.1363 & 0.2517 & 0.1361 & 0.2514 & 0.001379
\end{tabular}

$\begin{array}{lllll}0.6043 & 0.2571 & 0.6026 & 0.2564 & 0.002765 \\ 0.4049 & 0.3828 & 0.4040 & 0.3820 & 0.002154 \\ 0.0936 & 0.3132 & 0.0935 & 0.3128 & 0.001305 \\ 0.0635 & 0.7636 & 0.0634 & 0.7628 & 0.000994 \\ 0.1801 & 0.1888 & 0.1798 & 0.1885 & 0.001505 \\ 0.0825 & 0.5438 & 0.0824 & 0.5432 & 0.001189 \\ 0.3591 & 0.1860 & 0.3584 & 0.1856 & 0.001937 \\ 0.4449 & 0.1868 & 0.4439 & 0.1864 & 0.002213\end{array}$

${ }^{\mathrm{a}} x_{3}{ }^{(\mathrm{s})}$ : initial mole fraction of component 3 in the ternary solvent mixture, $x_{4}{ }^{(\mathrm{s})}$ : initial mole fraction of component 4 in the ternary solvent mixture; $x_{1}$ : mole fraction solubility of the solute; $x_{3}$ : mole fraction of component 3 in the quaternary solution; $x_{4}$ : mole fraction of component 4 in the quaternary solution.

\section{Auxiliary Information}

\section{Method/Apparatus/Procedure:}

Constant-temperature bath, calorimetric thermometer, and an ultraviolet/ visible spectrophotometer.

Ternary solvent mixtures were prepared by mass. Excess solute and solvent were placed in amber glass bottles and allowed to equilibrate for several days at constant temperature. Attainment of equilibrium was verified by several repetitive measurements and by approaching equilibrium from supersaturation. Aliquots of saturated solutions were transferred through a coarse filter into volumetric flasks, weighed and diluted with methanol. Molar concentrations were determined by spectrophotometric measurements at $356 \mathrm{~nm}$.

Source and Purity of Chemicals:

(1) $99+\%$, Acros Chemical Company, USA, was recrystallized three times from acetone.

(2) $99+\%$, HPLC grade, Aldrich Chemical Company, Milwaukee, WI, USA.

(3) $99.3 \%$, anhydrous, Aldrich Chemical Company.

(4) $99+\%$, anhydrous, Aldrich Chemical Company.

Components 2-4 were stored over molecular sieves and distilled shortly before use.

Estimated Error:

Temperature: $\pm 0.1 \mathrm{~K}$.

$x_{3}{ }^{(\mathrm{s})}: \pm 0.0001$.

$x_{4}{ }^{(\mathrm{s})}: \pm 0.0001$.

$x_{1}: \pm 1.5 \%$ (relative error).

\begin{tabular}{|c|c|}
\hline $\begin{array}{l}\text { Components: } \\
\text { (1) Anthracene; } \mathrm{C}_{14} \mathrm{H}_{10} ;[120-12-7] \\
\text { (2) } 2,2,4-\text { Trimethylpentane; } \mathrm{C}_{8} \mathrm{H}_{18} \\
\text { [540-84-1] } \\
\text { (3) } 1,1^{\prime} \text {-Oxybisbutane; } \mathrm{C}_{8} \mathrm{H}_{18} \mathrm{O} ; \\
\text { [142-96-1] } \\
\text { (4) 2-Methyl-1-propanol; } \mathrm{C}_{4} \mathrm{H}_{10} \mathrm{O} \text {; } \\
\text { [78-83-1] }\end{array}$ & $\begin{array}{l}\text { Original Measurements: } \\
{ }^{19} \text { K. J. Pribyla, M. A. Spurgin, I. } \\
\text { Chuca, and W. E. Acree, Jr., J. } \\
\text { Chem. Eng. Data 44, } 1265 \text { (1999). }\end{array}$ \\
\hline $\begin{array}{l}\text { Variables: } \\
T / \mathrm{K}=298.15 ; \text { Solvent Composition }\end{array}$ & $\begin{array}{l}\text { Prepared by: } \\
\text { W. E. Acree, Jr. }\end{array}$ \\
\hline
\end{tabular}


Experimental Values

Solubility of anthracene in 2,2,4-trimethylpentane $+1,1^{\prime}$-oxybisbutane +2 methyl-1-propanol mixtures ${ }^{\mathrm{a}}$

\begin{tabular}{lcccc}
\hline \hline$x_{3}{ }^{(\mathrm{s})}$ & $x_{4}{ }^{(\mathrm{s})}$ & $x_{3}$ & $x_{4}$ & $x_{1}$ \\
\hline 0.0000 & 0.0000 & 0.0000 & 0.0000 & 0.001074 \\
1.0000 & 0.0000 & 0.9964 & 0.0000 & 0.003615 \\
0.0000 & 1.0000 & 0.0000 & 0.9995 & 0.000470 \\
0.2585 & 0.4763 & 0.2581 & 0.4755 & 0.001585 \\
0.0982 & 0.7999 & 0.0981 & 0.7992 & 0.000878 \\
0.2043 & 0.3769 & 0.2040 & 0.3763 & 0.001471 \\
0.1784 & 0.6378 & 0.1782 & 0.6370 & 0.001246 \\
0.6270 & 0.1809 & 0.6253 & 0.1804 & 0.002734 \\
0.5870 & 0.3217 & 0.5855 & 0.3209 & 0.002615 \\
0.1664 & 0.7408 & 0.1662 & 0.7400 & 0.001097 \\
0.0766 & 0.6373 & 0.0765 & 0.6367 & 0.000980 \\
0.2818 & 0.6390 & 0.2814 & 0.6380 & 0.001529 \\
0.3696 & 0.5440 & 0.3689 & 0.5430 & 0.001871 \\
0.1332 & 0.2526 & 0.1330 & 0.2523 & 0.001348 \\
0.6055 & 0.2554 & 0.6039 & 0.2547 & 0.002708 \\
0.4056 & 0.3856 & 0.4048 & 0.3848 & 0.002051 \\
0.0922 & 0.3174 & 0.0921 & 0.3170 & 0.001217 \\
0.0646 & 0.8027 & 0.0645 & 0.8021 & 0.000798 \\
0.1823 & 0.1789 & 0.1820 & 0.1786 & 0.001462 \\
0.0775 & 0.5460 & 0.0774 & 0.5454 & 0.001056 \\
0.3585 & 0.1807 & 0.3578 & 0.1804 & 0.001916 \\
0.4490 & 0.1806 & 0.4480 & 0.1802 & 0.002187 \\
\hline
\end{tabular}

${ }^{\mathrm{a}} x_{3}{ }^{(\mathrm{s})}$ : initial mole fraction of component 3 in the ternary solvent mixture, $x_{4}{ }^{(\mathrm{s})}$. initial mole fraction of component 4 in the ternary solvent mixture; $x_{1}$ : mole fraction solubility of the solute; $x_{3}$ : mole fraction of component 3 in the quaternary solution; $x_{4}$ : mole fraction of component 4 in the quaternary solution.

\subsection{Anthracene solubility data in ternary alkane +2 - methoxy-2-methylpropane + alcohol solvent mixtures}

\begin{tabular}{|c|c|}
\hline $\begin{array}{l}\text { Components: } \\
\text { (1) Anthracene; } \mathrm{C}_{14} \mathrm{H}_{10} ;[120-12-7] \\
\text { (2) Heptane; } \mathrm{C}_{7} \mathrm{H}_{16} ;[142-82-5] \\
\text { (3) 2-Methoxy-2-methylpropane; } \\
\mathrm{C}_{5} \mathrm{H}_{12} \mathrm{O} ;[1634-04-4] \\
\text { (4) 1-Propanol; } \mathrm{C}_{3} \mathrm{H}_{8} \mathrm{O} ;[\text { [71-23-8] }\end{array}$ & $\begin{array}{l}\text { Original Measurements: } \\
{ }^{18} \text { K. J. Pribyla, I. Chuca, T. T. } \\
\text { Van, and W. E. Acree, Jr., J. } \\
\text { Chem. Eng. Data 45, } 533 \text { (2000). }\end{array}$ \\
\hline $\begin{array}{l}\text { Variables: } \\
T / \mathrm{K}=298.15 ; \text { Solvent Composition }\end{array}$ & $\begin{array}{l}\text { Prepared by: } \\
\text { W. E. Acree, Jr. }\end{array}$ \\
\hline
\end{tabular}

Experimental Values

Solubility of anthracene in heptane +2 -methoxy-2-methylpropane +1 -propanol mixtures ${ }^{\mathrm{a}}$

\begin{tabular}{lcccc}
\hline \hline$x_{3}{ }^{(\mathrm{s})}$ & $x_{4}{ }^{(\mathrm{s})}$ & $x_{3}$ & $x_{4}$ & $x_{1}$ \\
\hline 0.0000 & 0.0000 & 0.0000 & 0.0000 & 0.001571 \\
1.0000 & 0.0000 & 0.9969 & 0.0000 & 0.003050 \\
0.0000 & 1.0000 & 0.0000 & 0.9994 & 0.000591 \\
0.2947 & 0.4682 & 0.2942 & 0.4674 & 0.001808 \\
0.1100 & 0.7996 & 0.1099 & 0.7988 & 0.001041 \\
0.2370 & 0.3877 & 0.2366 & 0.3870 & 0.001772 \\
0.2008 & 0.6364 & 0.2006 & 0.6355 & 0.001430 \\
0.6685 & 0.1665 & 0.6668 & 0.1661 & 0.002606 \\
0.6234 & 0.2953 & 0.6219 & 0.2946 & 0.002455 \\
0.1479 & 0.7864 & 0.1477 & 0.7855 & 0.001099 \\
0.0972 & 0.6397 & 0.0971 & 0.6389 & 0.001274 \\
0.3048 & 0.6128 & 0.3043 & 0.6118 & 0.001584 \\
0.4049 & 0.5218 & 0.4041 & 0.5208 & 0.001860 \\
0.1617 & 0.2541 & 0.1614 & 0.2537 & 0.001747 \\
0.6442 & 0.2377 & 0.6426 & 0.2371 & 0.002504 \\
0.4380 & 0.3753 & 0.4370 & 0.3745 & 0.002112 \\
0.1122 & 0.3485 & 0.1120 & 0.3479 & 0.001620 \\
0.0817 & 0.8003 & 0.0816 & 0.7995 & 0.001007 \\
0.2252 & 0.1761 & 0.2248 & 0.1758 & 0.001906 \\
0.1045 & 0.5491 & 0.1044 & 0.5483 & 0.001400 \\
0.4171 & 0.1774 & 0.4162 & 0.1770 & 0.002241 \\
0.5027 & 0.1723 & 0.5015 & 0.1719 & 0.002364 \\
\hline
\end{tabular}

${ }^{a_{3}}{ }_{3}{ }^{(\mathrm{s})}$ : initial mole fraction of component 3 in the ternary solvent mixture, $x_{4}{ }^{(\mathrm{s})}$ : initial mole fraction of component 4 in the ternary solvent mixture; $x_{1}$ : mole fraction solubility of the solute; $x_{3}$ : mole fraction of component 3 in the quaternary solution; $x_{4}$ : mole fraction of component 4 in the quaternary solution.

\section{Auxiliary Information} from acetone.

(2) $99+\%$, HPLC grade, Aldrich Chemical Company, Milwaukee, WI, USA. (3) $99.3 \%$, anhydrous, Aldrich Chemical Company.

(4) $99+\%$, anhydrous, Aldrich Chemical Company.

Components $2-4$ were stored over molecular sieves and distilled shortly before use.

\section{Estimated Error:}

Temperature: $\pm 0.1 \mathrm{~K}$.

$x_{3}^{(\mathrm{s})}: \pm 0.0001$.

$x_{4}{ }^{(\mathrm{s})}: \pm 0.0001$

$x_{1}: \pm 1.5 \%$ (relative error).

\section{Method/Apparatus/Procedure:}

Constant-temperature bath, calorimetric thermometer, and an ultraviolet/ visible spectrophotometer.

Ternary solvent mixtures were prepared by mass. Excess solute and solvent were placed in amber glass bottles and allowed to equilibrate for several days at constant temperature. Attainment of equilibrium was verified by several repetitive measurements and by approaching equilibrium from supersaturation. Aliquots of saturated solutions were transferred through a coarse filter into volumetric flasks, weighed and diluted with methanol. Molar concentrations were determined by spectrophotometric measurements at $356 \mathrm{~nm}$. 
Source and Purity of Chemicals:

(1) $99+\%$, Aldrich Chemical Company, Milwaukee, WI, USA, was used as received.

(2) $99+\%$, HPLC grade, Aldrich Chemical Company.

(3) $99.9 \%$, Arco Chemical Company, USA.

(4) $99+\%$, anhydrous, Aldrich Chemical Company.

Components $2-4$ were stored over molecular sieves and distilled shortly before use.

\section{Estimated Error:}

Temperature: $\pm 0.1 \mathrm{~K}$

$x_{3}{ }^{(\mathrm{s})}: \pm 0.0001$.

$x_{4}{ }^{(\mathrm{s})}: \pm 0.0001$

$x_{1}: \pm 1.5 \%$ (relative error).

\section{Components:}

(1) Anthracene; $\mathrm{C}_{14} \mathrm{H}_{10} ;[120-12-7]$

(2) Heptane; $\mathrm{C}_{7} \mathrm{H}_{16} ;[142-82-5]$

(3) 2-Methoxy-2-methylpropane;

$\mathrm{C}_{5} \mathrm{H}_{12} \mathrm{O}$; [1634-04-4]

(4) 2-Propanol; $\mathrm{C}_{3} \mathrm{H}_{8} \mathrm{O}$; [67-63-0]

\begin{tabular}{ll}
\hline Variables: & Prepared by: \\
$T / \mathrm{K}=298.15$; Solvent Composition & W. E. Acree, Jr.
\end{tabular}

\section{Experimental Values}

Solubility of anthracene in heptane +2 -methoxy-2-methylpropane +2 -propanol mixtures ${ }^{\mathrm{a}}$

\begin{tabular}{|c|c|c|c|c|}
\hline$x_{3}{ }^{(\mathrm{s})}$ & $x_{4}{ }^{(\mathrm{s})}$ & $x_{3}$ & $x_{4}$ & $x_{1}$ \\
\hline 0.0000 & 0.0000 & 0.0000 & 0.0000 & 0.001571 \\
\hline 1.0000 & 0.0000 & 0.9969 & 0.0000 & 0.003050 \\
\hline 0.0000 & 1.0000 & 0.0000 & 0.9996 & 0.000411 \\
\hline 0.2981 & 0.4593 & 0.2976 & 0.4585 & 0.001647 \\
\hline 0.1152 & 0.7896 & 0.1151 & 0.7889 & 0.000851 \\
\hline 0.2456 & 0.3720 & 0.2452 & 0.3714 & 0.001677 \\
\hline 0.2049 & 0.6272 & 0.2046 & 0.6264 & 0.001235 \\
\hline 0.6691 & 0.1703 & 0.6674 & 0.1699 & 0.002549 \\
\hline 0.6300 & 0.2941 & 0.6285 & 0.2934 & 0.002436 \\
\hline 0.1504 & 0.7829 & 0.1503 & 0.7822 & 0.000893 \\
\hline 0.0924 & 0.6382 & 0.0923 & 0.6375 & 0.001106 \\
\hline 0.3091 & 0.6181 & 0.3087 & 0.6172 & 0.001385 \\
\hline 0.4051 & 0.5175 & 0.4044 & 0.5166 & 0.001670 \\
\hline 0.1605 & 0.2579 & 0.1602 & 0.2575 & 0.001648 \\
\hline 0.6447 & 0.2380 & 0.6431 & 0.2374 & 0.002438 \\
\hline 0.4527 & 0.3594 & 0.4518 & 0.3587 & 0.001997 \\
\hline 0.1123 & 0.3186 & 0.1121 & 0.3181 & 0.001529 \\
\hline 0.0848 & 0.7918 & 0.0847 & 0.7911 & 0.000828 \\
\hline 0.2166 & 0.1846 & 0.2162 & 0.1843 & 0.001818 \\
\hline 0.0969 & 0.5478 & 0.0968 & 0.5471 & 0.001254 \\
\hline 0.4052 & 0.1804 & 0.4043 & 0.1800 & 0.002107 \\
\hline 0.4890 & 0.1818 & 0.4879 & 0.1814 & 0.002256 \\
\hline
\end{tabular}

${ }^{\mathrm{a}} x_{3}{ }^{(\mathrm{s})}$ : initial mole fraction of component 3 in the ternary solvent mixture, $x_{4}{ }^{(\mathrm{s})}$ : initial mole fraction of component 4 in the ternary solvent mixture; $x_{1}$ : mole fraction solubility of the solute; $x_{3}$ : mole fraction of component 3 in the quaternary solution; $x_{4}$ : mole fraction of component 4 in the quaternary solution.

\section{Auxiliary Information}

Method/Apparatus/Procedure:

Constant-temperature bath, calorimetric thermometer, and an ultraviolet/ visible spectrophotometer.

Ternary solvent mixtures were prepared by mass. Excess solute and solvent were placed in amber glass bottles and allowed to equilibrate for several days at constant temperature. Attainment of equilibrium was verified by several repetitive measurements and by approaching equilibrium from supersaturation. Aliquots of saturated solutions were transferred through a coarse filter into volumetric flasks, weighed and diluted with methanol. Molar concentrations were determined by spectrophotometric measurements at $356 \mathrm{~nm}$.

Source and Purity of Chemicals:

(1) $99+\%$, Aldrich Chemical Company, Milwaukee, WI, USA, was used as received.

(2) $99+\%$, HPLC grade, Aldrich Chemical Company.

(3) $99.9 \%$, Arco Chemical Company, USA.

(4) $99+\%$, anhydrous, Aldrich Chemical Company.

Components $2-4$ were stored over molecular sieves and distilled shortly before use.

Estimated Error:

Temperature: $\pm 0.1 \mathrm{~K}$.

$x_{3}{ }^{(\mathrm{s})}: \pm 0.0001$.

$x_{4}{ }^{(\mathrm{s})}: \pm 0.0001$.

$x_{1}: \pm 1.5 \%$ (relative error).

\begin{tabular}{ll}
\hline \hline Components: & Original Measurements: \\
(1) Anthracene; $\mathrm{C}_{14} \mathrm{H}_{10} ;[120-12-7]$ & ${ }^{18} \mathrm{~K}$. J. Pribyla, I. Chuca, T. T. \\
(2) Heptane; $\mathrm{C}_{7} \mathrm{H}_{16} ;[142-82-5]$ & Van, and W. E. Acree, Jr., J. \\
(3) 2-Methoxy-2-methylpropane; & Chem. Eng. Data 45, 533 (2000). \\
$\mathrm{C}_{5} \mathrm{H}_{12} \mathrm{O} ;[1634-04-4]$ & \\
(4) 1-Butanol; $\mathrm{C}_{4} \mathrm{H}_{10} \mathrm{O} ;[71-36-3]$ & \\
\hline Variables: & Prepared by: \\
$T / \mathrm{K}=298.15 ;$ Solvent Composition & W. E. Acree, Jr. \\
\hline
\end{tabular}




\section{Experimental Values}

Solubility of anthracene in heptane +2 -methoxy-2-methylpropane +1 -butanol mixtures $^{\mathrm{a}}$

\begin{tabular}{lcccc}
\hline \hline$x_{3}{ }^{(\mathrm{s})}$ & $x_{4}{ }^{(\mathrm{s})}$ & $x_{3}$ & $x_{4}$ & $x_{1}$ \\
\hline 0.0000 & 0.0000 & 0.0000 & 0.0000 & 0.001571 \\
1.0000 & 0.0000 & 0.9969 & 0.0000 & 0.003050 \\
0.0000 & 1.0000 & 0.0000 & 0.9992 & 0.000801 \\
0.3186 & 0.4215 & 0.3180 & 0.4207 & 0.001951 \\
0.1244 & 0.7650 & 0.1242 & 0.7640 & 0.001290 \\
0.2514 & 0.3428 & 0.2509 & 0.3422 & 0.001888 \\
0.2343 & 0.6079 & 0.2339 & 0.6069 & 0.001646 \\
0.6864 & 0.1531 & 0.6846 & 0.1527 & 0.002672 \\
0.6494 & 0.2620 & 0.6477 & 0.2613 & 0.002589 \\
0.1646 & 0.7591 & 0.1644 & 0.7581 & 0.001334 \\
0.1017 & 0.6011 & 0.1016 & 0.6002 & 0.001444 \\
0.3504 & 0.5685 & 0.3497 & 0.5674 & 0.001888 \\
0.4497 & 0.4698 & 0.4486 & 0.4688 & 0.002124 \\
0.1650 & 0.2237 & 0.1647 & 0.2233 & 0.001808 \\
0.6740 & 0.1968 & 0.6722 & 0.1963 & 0.002660 \\
0.4775 & 0.3220 & 0.4764 & 0.3213 & 0.002315 \\
0.1085 & 0.2898 & 0.1083 & 0.2893 & 0.001703 \\
0.0924 & 0.7674 & 0.0923 & 0.7664 & 0.001247 \\
0.2210 & 0.1638 & 0.2206 & 0.1635 & 0.001908 \\
0.1105 & 0.5035 & 0.1103 & 0.5027 & 0.001555 \\
0.4260 & 0.1563 & 0.4250 & 0.1559 & 0.002281 \\
0.5097 & 0.1507 & 0.5085 & 0.1503 & 0.002406 \\
\hline$x_{3}(\mathrm{~s}: 15150$
\end{tabular}

${ }^{\mathrm{a}} x_{3}{ }^{(\mathrm{s})}$ : initial mole fraction of component 3 in the ternary solvent mixture, $x_{4}{ }^{(\mathrm{s})}$ : initial mole fraction of component 4 in the ternary solvent mixture; $x_{1}$ : mole fraction solubility of the solute; $x_{3}$ : mole fraction of component 3 in the quaternary solution; $x_{4}$ : mole fraction of component 4 in the quaternary solution.

\section{Auxiliary Information}

\section{Method/Apparatus/Procedure:}

Constant-temperature bath, calorimetric thermometer, and an ultraviolet/ visible spectrophotometer.

Ternary solvent mixtures were prepared by mass. Excess solute and solvent were placed in amber glass bottles and allowed to equilibrate for several days at constant temperature. Attainment of equilibrium was verified by several repetitive measurements and by approaching equilibrium from supersaturation. Aliquots of saturated solutions were transferred through a coarse filter into volumetric flasks, weighed and diluted with methanol. Molar concentrations were determined by spectrophotometric measurements at $356 \mathrm{~nm}$.

\section{Source and Purity of Chemicals:}

(1) $99+\%$, Aldrich Chemical Company, Milwaukee, WI, USA, was used as received.

(2) $99+\%$, HPLC grade, Aldrich Chemical Company.

(3) $99.9 \%$, Arco Chemical Company, USA.

(4) $99.8+\%$, HPLC grade, Aldrich Chemical Company.

Components 2-4 were stored over molecular sieves and distilled shortly before use.

\section{Estimated Error:}

Temperature: $\pm 0.1 \mathrm{~K}$

$x_{3}^{(\mathrm{s})}: \pm 0.0001$.

$x_{4}{ }^{(\mathrm{s})}: \pm 0.0001$.

$x_{1}: \pm 1.5 \%$ (relative error).

\begin{tabular}{ll}
\hline \hline Components: & Original Measurements: \\
(1) Anthracene; $\mathrm{C}_{14} \mathrm{H}_{10} ;[120-12-7]$ & ${ }^{18} \mathrm{~K}$. J. Pribyla, I. Chuca, T. T. \\
(2) Heptane; $\mathrm{C}_{7} \mathrm{H}_{16} ;[142-82-5]$ & Van, and W. E. Acree, Jr., J. \\
(3) 2-Methoxy-2-methylpropane; & Chem. Eng. Data 45, 533 (2000). \\
$\mathrm{C}_{5} \mathrm{H}_{12} \mathrm{O} ;[1634-04-4]$ & \\
(4) 2-Butanol; $\mathrm{C}_{4} \mathrm{H}_{10} \mathrm{O} ;[78-92-2]$ & \\
\hline Variables: & Prepared by: \\
$T / \mathrm{K}=298.15 ;$ Solvent Composition & W. E. Acree, Jr. \\
\hline
\end{tabular}

Experimental Values

Solubility of anthracene in heptane +2 -methoxy-2-methylpropane +2 -butanol mixtures $^{\mathrm{a}}$

\begin{tabular}{lcccc}
\hline \hline$x_{3}{ }^{(\mathrm{s})}$ & $x_{4}{ }^{(\mathrm{s})}$ & $x_{3}$ & $x_{4}$ & $x_{1}$ \\
\hline 0.0000 & 0.0000 & 0.0000 & 0.0000 & 0.001571 \\
1.0000 & 0.0000 & 0.9969 & 0.0000 & 0.003050 \\
0.0000 & 1.0000 & 0.0000 & 0.9994 & 0.000585 \\
0.3189 & 0.4228 & 0.3183 & 0.4220 & 0.001853 \\
0.1326 & 0.7613 & 0.1325 & 0.7605 & 0.001104 \\
0.2637 & 0.3344 & 0.2632 & 0.3338 & 0.001880 \\
0.2319 & 0.5806 & 0.2316 & 0.5797 & 0.001501 \\
0.6940 & 0.1418 & 0.6922 & 0.1414 & 0.002665 \\
0.6557 & 0.2575 & 0.6540 & 0.2568 & 0.002529 \\
0.1704 & 0.7523 & 0.1702 & 0.7514 & 0.001165 \\
0.1045 & 0.5936 & 0.1044 & 0.5928 & 0.001297 \\
0.3546 & 0.5662 & 0.3540 & 0.5652 & 0.001691 \\
0.4394 & 0.4763 & 0.4385 & 0.4754 & 0.001935 \\
0.1649 & 0.2250 & 0.1646 & 0.2246 & 0.001796 \\
0.6730 & 0.1997 & 0.6712 & 0.1992 & 0.002615 \\
0.4787 & 0.3215 & 0.4776 & 0.3208 & 0.002221 \\
0.1207 & 0.2804 & 0.1205 & 0.2799 & 0.001659 \\
0.0938 & 0.7647 & 0.0937 & 0.7639 & 0.001041 \\
0.2274 & 0.1576 & 0.2270 & 0.1573 & 0.001922 \\
0.1113 & 0.5096 & 0.1111 & 0.5089 & 0.001425 \\
0.4222 & 0.1570 & 0.4213 & 0.1567 & 0.002224 \\
0.5136 & 0.1474 & 0.5124 & 0.1470 & 0.002376 \\
\hline
\end{tabular}

${ }^{\mathrm{a}} x_{3}{ }^{(\mathrm{s})}$ : initial mole fraction of component 3 in the ternary solvent mixture, $x_{4}{ }^{(\mathrm{s})}$ : initial mole fraction of component 4 in the ternary solvent mixture; $x_{1}$ : mole fraction solubility of the solute; $x_{3}$ : mole fraction of component 3 in the quaternary solution; $x_{4}$ : mole fraction of component 4 in the quaternary solution.

\section{Auxiliary Information}

\section{Method/Apparatus/Procedure:}

Constant-temperature bath, calorimetric thermometer, and an ultraviolet/ visible spectrophotometer.

Ternary solvent mixtures were prepared by mass. Excess solute and solvent were placed in amber glass bottles and allowed to equilibrate for several days at constant temperature. Attainment of equilibrium was verified by several repetitive measurements and by approaching equilibrium from supersaturation. Aliquots of saturated solutions were transferred through a coarse filter into volumetric flasks, weighed and diluted with methanol. Molar concentrations were determined by spectrophotometric measurements at $356 \mathrm{~nm}$.

Source and Purity of Chemicals:

(1) $99+\%$, Aldrich Chemical Company, Milwaukee, WI, USA, was used as received.

(2) $99+\%$, HPLC grade, Aldrich Chemical Company.

(3) $99.9 \%$, Arco Chemical Company, USA.

(4) $99+\%$, anhydrous, Aldrich Chemical Company.

Components 2-4 were stored over molecular sieves and distilled shortly before use. 
Estimated Error:

Temperature: $\pm 0.1 \mathrm{~K}$.

$x_{3}{ }^{(\mathrm{s})}: \pm 0.0001$.

$x_{4}{ }^{(\mathrm{s})}: \pm 0.0001$.

$x_{1}: \pm 1.5 \%$ (relative error).

\begin{tabular}{ll}
\hline \hline Components: & Original Measurements: \\
(1) Anthracene; $\mathrm{C}_{14} \mathrm{H}_{10} ;[120-12-7]$ & ${ }^{18} \mathrm{~K}$. J. Pribyla, I. Chuca, T. T. \\
(2) Heptane; $\mathrm{C}_{7} \mathrm{H}_{16} ;[142-82-5]$ & Van, and W. E. Acree, Jr., J. \\
(3) 2-Methoxy-2-methylpropane; & Chem. Eng. Data 45, 533 (2000). \\
$\mathrm{C}_{5} \mathrm{H}_{12} \mathrm{O} ;[1634-04-4]$ & \\
(4) 2-Methyl-1-propanol; $\mathrm{C}_{4} \mathrm{H}_{10} \mathrm{O} ;$ & \\
[78-83-1] & \\
\hline Variables: & Prepared by: \\
$T / \mathrm{K}=298.15 ;$ Solvent Composition & W. E. Acree, Jr. \\
\hline
\end{tabular}

Experimental Values

Solubility of anthracene in heptane +2 -methoxy-2-methylpropane +2 methyl-1-propanol mixtures ${ }^{\mathrm{a}}$

\begin{tabular}{lcccc}
\hline \hline$x_{3}{ }^{(\mathrm{s})}$ & $x_{4}{ }^{(\mathrm{s})}$ & $x_{3}$ & $x_{4}$ & $x_{1}$ \\
\hline 0.0000 & 0.0000 & 0.0000 & 0.0000 & 0.001571 \\
1.0000 & 0.0000 & 0.9969 & 0.0000 & 0.003050 \\
0.0000 & 1.0000 & 0.0000 & 0.9995 & 0.000470 \\
0.3156 & 0.4155 & 0.3151 & 0.4148 & 0.001739 \\
0.1463 & 0.7468 & 0.1462 & 0.7461 & 0.000997 \\
0.2614 & 0.3293 & 0.2609 & 0.3287 & 0.001782 \\
0.2333 & 0.5789 & 0.2330 & 0.5781 & 0.001371 \\
0.6904 & 0.1447 & 0.6886 & 0.1443 & 0.002521 \\
0.6657 & 0.2504 & 0.6641 & 0.2598 & 0.002359 \\
0.1724 & 0.7503 & 0.1722 & 0.7496 & 0.000994 \\
0.1072 & 0.5909 & 0.1071 & 0.5902 & 0.001205 \\
0.3531 & 0.5647 & 0.3526 & 0.5638 & 0.001549 \\
0.4487 & 0.4690 & 0.4479 & 0.4681 & 0.001813 \\
0.1707 & 0.2189 & 0.1704 & 0.2185 & 0.001758 \\
0.6803 & 0.2013 & 0.6786 & 0.2008 & 0.002431 \\
0.4752 & 0.3194 & 0.4742 & 0.3187 & 0.002069 \\
0.1239 & 0.2790 & 0.1237 & 0.2786 & 0.001604 \\
0.0943 & 0.7631 & 0.0942 & 0.7624 & 0.000910 \\
0.2230 & 0.1799 & 0.2226 & 0.1796 & 0.001854 \\
0.1141 & 0.4995 & 0.1139 & 0.4988 & 0.001348 \\
0.4188 & 0.1582 & 0.4179 & 0.1579 & 0.002172 \\
0.5096 & 0.1539 & 0.5084 & 0.1536 & 0.002273 \\
\hline${ }^{a} x_{3}{ }^{(s)}:$ initial mole fraction of component 3 in the ternary solvent mixture, $x_{4}{ }^{(\mathrm{s})}$ : \\
initial mole fraction of component 4 in the ternary solvent mixture; $x_{1}:$ mole \\
fraction solubility of the solute; $x_{3}:$ mole fraction of component 3 in the \\
quaternary solution $; x_{4}:$ mole fraction of component 4 in the quaternary \\
solution. & & & & \\
& & & &
\end{tabular}

\section{Auxiliary Information}

\footnotetext{
Method/Apparatus/Procedure:

Constant-temperature bath, calorimetric thermometer, and an ultraviolet/ visible spectrophotometer.

Ternary solvent mixtures were prepared by mass. Excess solute and solvent were placed in amber glass bottles and allowed to equilibrate for several days at constant temperature. Attainment of equilibrium was verified by several repetitive measurements and by approaching equilibrium from supersaturation. Aliquots of saturated solutions were transferred through a coarse filter into volumetric flasks, weighed and diluted with methanol. Molar concentrations were determined by spectrophotometric measurements at $356 \mathrm{~nm}$.
}

Source and Purity of Chemicals:

(1) $99+\%$, Aldrich Chemical Company, Milwaukee, WI, USA, was used as received.

(2) $99+\%$, HPLC grade, Aldrich Chemical Company.

(3) $99.9 \%$, Arco Chemical Company, USA.

(4) $99+\%$, anhydrous, Aldrich Chemical Company.

Components 2-4 were stored over molecular sieves and distilled shortly before use.

\section{Estimated Error:}

Temperature: $\pm 0.1 \mathrm{~K}$.

$x_{3}{ }^{(s)}: \pm 0.0001$.

$x_{4}{ }^{(\mathrm{s})}: \pm 0.0001$.

$x_{1}: \pm 1.5 \%$ (relative error).

\begin{tabular}{ll}
\hline \hline Components: & Original Measurements: \\
(1) Anthracene; $\mathrm{C}_{14} \mathrm{H}_{10} ;[120-12-7]$ & ${ }^{17}$ K. J. Pribyla, M. A. Spurgin, I. \\
(2) Cyclohexane; $\mathrm{C}_{6} \mathrm{H}_{12} ;[110-82-7]$ & Chuca, and W. E. Acree, Jr., J. \\
(3) 2-Methoxy-2-methylpropane; & Chem. Eng. Data 45, 530 (2000). \\
$\mathrm{C}_{5} \mathrm{H}_{12} \mathrm{O}$; [1634-04-4] &
\end{tabular}

(4) 1-Propanol; $\mathrm{C}_{3} \mathrm{H}_{8} \mathrm{O}$; [71-23-8]

\begin{tabular}{ll}
\hline Variables: & Prepared by: \\
$T / \mathrm{K}=298.15 ;$ Solvent Composition & W. E. Acree, Jr. \\
\hline
\end{tabular}

Experimental Values

Solubility of anthracene in cyclohexane +2 -methoxy-2-methylpropane +1 propanol mixtures ${ }^{\mathrm{a}}$

\begin{tabular}{lcccc}
\hline \hline$x_{3}{ }^{(\mathrm{s})}$ & $x_{4}{ }^{(\mathrm{s})}$ & $x_{3}$ & $x_{4}$ & $x_{1}$ \\
\hline 0.0000 & 0.0000 & 0.0000 & 0.0000 & 0.001553 \\
1.0000 & 0.0000 & 0.9969 & 0.0000 & 0.003050 \\
0.0000 & 1.0000 & 0.0000 & 0.9994 & 0.000591 \\
0.3609 & 0.3797 & 0.3601 & 0.3789 & 0.002108 \\
0.1106 & 0.7642 & 0.1105 & 0.7634 & 0.001097 \\
0.2156 & 0.3313 & 0.2152 & 0.3307 & 0.001919 \\
0.1861 & 0.5961 & 0.1858 & 0.5952 & 0.001505 \\
0.6372 & 0.1605 & 0.6354 & 0.1601 & 0.002763 \\
0.6054 & 0.2827 & 0.6039 & 0.2820 & 0.002527 \\
0.1427 & 0.7713 & 0.1425 & 0.7704 & 0.001139 \\
0.0837 & 0.5888 & 0.0836 & 0.5880 & 0.001309 \\
0.3018 & 0.5987 & 0.3013 & 0.5977 & 0.001640 \\
0.3884 & 0.5038 & 0.3877 & 0.5028 & 0.001926 \\
0.1254 & 0.2070 & 0.1252 & 0.2066 & 0.001847 \\
0.4682 & 0.2435 & 0.4671 & 0.2429 & 0.002454 \\
0.4188 & 0.3410 & 0.4179 & 0.3402 & 0.002234 \\
0.0928 & 0.2731 & 0.0926 & 0.2726 & 0.001711 \\
0.0766 & 0.7673 & 0.0765 & 0.7665 & 0.001040 \\
0.1829 & 0.1550 & 0.1825 & 0.1547 & 0.002009 \\
0.0858 & 0.4915 & 0.0857 & 0.4908 & 0.001458 \\
0.3584 & 0.1570 & 0.3576 & 0.1566 & 0.002355 \\
0.4554 & 0.1701 & 0.4543 & 0.1697 & 0.002509 \\
\hline$x_{3}: 1 n$ & 0.159 &
\end{tabular}

${ }^{\mathrm{a}} x_{3}{ }^{(\mathrm{s})}$ : initial mole fraction of component 3 in the ternary solvent mixture, $x_{4}{ }^{(\mathrm{s})}$ : initial mole fraction of component 4 in the ternary solvent mixture; $x_{1}$ : mole fraction solubility of the solute; $x_{3}$ : mole fraction of component 3 in the quaternary solution; $x_{4}$ : mole fraction of component 4 in the quaternary solution. 


\section{Auxiliary Information}

\section{Method/Apparatus/Procedure:}

Constant-temperature bath, calorimetric thermometer, and an ultraviolet/ visible spectrophotometer.

Ternary solvent mixtures were prepared by mass. Excess solute and solvent were placed in amber glass bottles and allowed to equilibrate for several days at constant temperature. Attainment of equilibrium was verified by several repetitive measurements and by approaching equilibrium from supersaturation. Aliquots of saturated solutions were transferred through a coarse filter into volumetric flasks, weighed and diluted with methanol. Molar concentrations were determined by spectrophotometric measurements at $356 \mathrm{~nm}$.

\section{Source and Purity of Chemicals:}

(1) $99.9+\%$, Aldrich Chemical Company, Milwaukee, WI, USA, was used as received.

(2) $99.9+\%$, HPLC grade, Aldrich Chemical Company.

(3) $99.9 \%$, Arco Chemical Company, USA.

(4) $99+\%$, anhydrous, Aldrich Chemical Company.

Components $2-4$ were stored over molecular sieves and distilled shortly before use.

\section{Estimated Error:}

Temperature: $\pm 0.1 \mathrm{~K}$

$x_{3}{ }^{(\mathrm{s})}: \pm 0.0001$.

$x_{4}{ }^{(\mathrm{s})}: \pm 0.0001$.

$x_{1}: \pm 1.5 \%$ (relative error).

\section{Components:}

(1) Anthracene; $\mathrm{C}_{14} \mathrm{H}_{10} ;[120-12-7]$

(2) Cyclohexane; $\mathrm{C}_{6} \mathrm{H}_{12} ;[110-82-7]$

(3) 2-Methoxy-2-methylpropane;

$\mathrm{C}_{5} \mathrm{H}_{12} \mathrm{O}$; [1634-04-4]

(4) 2-Propanol; $\mathrm{C}_{3} \mathrm{H}_{8} \mathrm{O}$; [67-63-0]

\begin{tabular}{ll}
\hline Variables: & Prepared by: \\
$T / \mathrm{K}=298.15 ;$ Solvent Composition & W. E. Acree, Jr. \\
\hline
\end{tabular}

\section{Experimental Values}

Solubility of anthracene in cyclohexane +2 -methoxy-2-methylpropane +2 propanol mixtures

\begin{tabular}{lcccc}
\hline \hline$x_{3}{ }^{(\mathrm{s})}$ & $x_{4}{ }^{(\mathrm{s})}$ & $x_{3}$ & $x_{4}$ & $x_{1}$ \\
\hline 0.0000 & 0.0000 & 0.0000 & 0.0000 & 0.001553 \\
1.0000 & 0.0000 & 0.9969 & 0.0000 & 0.003050 \\
0.0000 & 1.0000 & 0.0000 & 0.9996 & 0.000411 \\
0.2684 & 0.4257 & 0.2679 & 0.4249 & 0.001814 \\
0.1104 & 0.7651 & 0.1103 & 0.7644 & 0.000908 \\
0.2071 & 0.3401 & 0.2067 & 0.3395 & 0.001828 \\
0.1942 & 0.5880 & 0.1939 & 0.5872 & 0.001358 \\
0.6292 & 0.1609 & 0.6275 & 0.1605 & 0.002702 \\
0.6041 & 0.2785 & 0.6026 & 0.2778 & 0.002452 \\
0.1476 & 0.7665 & 0.1475 & 0.7658 & 0.000933 \\
0.0837 & 0.5791 & 0.0836 & 0.5783 & 0.001213 \\
0.3053 & 0.5976 & 0.3048 & 0.5967 & 0.001485 \\
0.3940 & 0.5050 & 0.3433 & 0.5041 & 0.001732 \\
0.1359 & 0.2086 & 0.1356 & 0.2082 & 0.001894
\end{tabular}

$\begin{array}{lllll}0.6158 & 0.2294 & 0.6142 & 0.2288 & 0.002543 \\ 0.4239 & 0.3334 & 0.4230 & 0.3327 & 0.002145 \\ 0.0917 & 0.2700 & 0.0915 & 0.2695 & 0.001695 \\ 0.0785 & 0.7589 & 0.0784 & 0.7582 & 0.000878 \\ 0.1798 & 0.1527 & 0.1794 & 0.1524 & 0.002003 \\ 0.0907 & 0.4852 & 0.0906 & 0.4845 & 0.001384 \\ 0.3556 & 0.1580 & 0.3548 & 0.1576 & 0.002374 \\ 0.4460 & 0.1585 & 0.4449 & 0.1581 & 0.002497\end{array}$

${ }^{\mathrm{a}} x_{3}{ }^{(\mathrm{s})}$ : initial mole fraction of component 3 in the ternary solvent mixture, $x_{4}{ }^{(\mathrm{s})}$ : initial mole fraction of component 4 in the ternary solvent mixture; $x_{1}$ : mole fraction solubility of the solute; $x_{3}$ : mole fraction of component 3 in the quaternary solution; $x_{4}$ : mole fraction of component 4 in the quaternary solution.

\section{Auxiliary Information}

\section{Method/Apparatus/Procedure:}

Constant-temperature bath, calorimetric thermometer, and an ultraviolet/ visible spectrophotometer.

Ternary solvent mixtures were prepared by mass. Excess solute and solvent were placed in amber glass bottles and allowed to equilibrate for several days at constant temperature. Attainment of equilibrium was verified by several repetitive measurements and by approaching equilibrium from supersaturation. Aliquots of saturated solutions were transferred through a coarse filter into volumetric flasks, weighed and diluted with methanol. Molar concentrations were determined by spectrophotometric measurements at $356 \mathrm{~nm}$.

Source and Purity of Chemicals:

(1) $99+\%$, Aldrich Chemical Company, Milwaukee, WI, USA, was used as received.

(2) $99.9+\%$, HPLC grade, Aldrich Chemical Company.

(3) $99.9 \%$, Arco Chemical Company, USA.

(4) $99+\%$, anhydrous, Aldrich Chemical Company.

Components $2-4$ were stored over molecular sieves and distilled shortly before use.

\section{Estimated Error:}

Temperature: $\pm 0.1 \mathrm{~K}$.

$x_{3}{ }^{(\mathrm{s})}: \pm 0.0001$.

$x_{4}{ }^{(\mathrm{s})}: \pm 0.0001$.

$x_{1}: \pm 1.5 \%$ (relative error).

\begin{tabular}{ll}
\hline \hline Components: & Original Measurements: \\
(1) Anthracene; $\mathrm{C}_{14} \mathrm{H}_{10} ;[120-12-7]$ & ${ }^{17} \mathrm{~K}$. J. Pribyla, M. A. Spurgin, I. \\
(2) Cyclohexane; $\mathrm{C}_{6} \mathrm{H}_{12} ;[110-82-7]$ & Chuca, and W. E. Acree, Jr., J. \\
(3) 2-Methoxy-2-methylpropane; & Chem. Eng. Data 45, 530 (2000). \\
$\mathrm{C}_{5} \mathrm{H}_{12} \mathrm{O} ;[1634-04-4]$ & \\
$(4) 1-\mathrm{Butanol} ; \mathrm{C}_{4} \mathrm{H}_{10} \mathrm{O} ;[71-36-3]$ & \\
\hline Variables: & Prepared by: \\
$T / \mathrm{K}=298.15 ;$ Solvent Composition & W. E. Acree, Jr. \\
\hline
\end{tabular}


Experimental Values

Solubility of anthracene in cyclohexane +2 -methoxy-2-methylpropane +1 butanol mixtures ${ }^{\mathrm{a}}$

\begin{tabular}{lcccc}
\hline \hline$x_{3}{ }^{(\mathrm{s})}$ & $x_{4}{ }^{(\mathrm{s})}$ & $x_{3}$ & $x_{4}$ & $x_{1}$ \\
\hline 0.0000 & 0.0000 & 0.0000 & 0.0000 & 0.001553 \\
1.0000 & 0.0000 & 0.9969 & 0.0000 & 0.003050 \\
0.0000 & 1.0000 & 0.0000 & 0.9992 & 0.000801 \\
0.2924 & 0.3834 & 0.2918 & 0.3826 & 0.002029 \\
0.1261 & 0.7353 & 0.1259 & 0.7343 & 0.001317 \\
0.2219 & 0.2995 & 0.2215 & 0.2989 & 0.002012 \\
0.2108 & 0.5456 & 0.2104 & 0.5446 & 0.001703 \\
0.6489 & 0.1337 & 0.6471 & 0.1333 & 0.002751 \\
0.6380 & 0.2493 & 0.6363 & 0.2486 & 0.002632 \\
0.1627 & 0.7392 & 0.1625 & 0.7382 & 0.001372 \\
0.0926 & 0.5394 & 0.0925 & 0.5386 & 0.001513 \\
0.3406 & 0.5590 & 0.3400 & 0.5579 & 0.001892 \\
0.4386 & 0.4583 & 0.4377 & 0.4573 & 0.002157 \\
0.1302 & 0.1867 & 0.1300 & 0.1863 & 0.001887 \\
0.6408 & 0.1929 & 0.6390 & 0.1924 & 0.002721 \\
0.4470 & 0.3027 & 0.4459 & 0.3020 & 0.002385 \\
0.0951 & 0.2279 & 0.0949 & 0.2275 & 0.001804 \\
0.0838 & 0.7343 & 0.0837 & 0.7334 & 0.001240 \\
0.1815 & 0.1329 & 0.1811 & 0.1326 & 0.002011 \\
0.0941 & 0.4406 & 0.0939 & 0.4399 & 0.001613 \\
0.3650 & 0.1327 & 0.3641 & 0.1324 & 0.002401 \\
0.4531 & 0.1367 & 0.4519 & 0.1363 & 0.002578 \\
\hline${ }^{\mathrm{a}}{ }_{3}{ }^{(\mathrm{s})}$ : initial mole fraction of component 3 in the ternary solvent mixture, $x_{4}{ }^{(\mathrm{s})}$ : \\
initial mole fraction of component 4 in the ternary solvent mixture; $x_{1}:$ mole \\
fraction solubility of the solute; $x_{3}:$ mole fraction of component 3 in the \\
quaternary solution; $x_{4}:$ mole fraction of component 4 in the quaternary \\
solution. & & & & \\
& & & &
\end{tabular}

\section{Auxiliary Information}

\section{Method/Apparatus/Procedure:}

Constant-temperature bath, calorimetric thermometer, and an ultraviolet/ visible spectrophotometer.

Ternary solvent mixtures were prepared by mass. Excess solute and solvent were placed in amber glass bottles and allowed to equilibrate for several days at constant temperature. Attainment of equilibrium was verified by several repetitive measurements and by approaching equilibrium from supersaturation. Aliquots of saturated solutions were transferred through a coarse filter into volumetric flasks, weighed and diluted with methanol. Molar concentrations were determined by spectrophotometric measurements at $356 \mathrm{~nm}$.

\section{Source and Purity of Chemicals:}

(1) $99+\%$, Aldrich Chemical Company, Milwaukee, WI, USA, was used as received.

(2) $99.9+\%$, HPLC grade, Aldrich Chemical Company, Milwaukee, WI, USA.

(3) $99.9 \%$, Arco Chemical Company, USA.

(4) $99.8+\%$, HPLC grade, Aldrich Chemical Company.

Components 2-4 were stored over molecular sieves and distilled shortly before use.

\section{Estimated Error:}

Temperature: $\pm 0.1 \mathrm{~K}$

$x_{3}^{(\mathrm{s})}: \pm 0.0001$.

$x_{4}{ }^{(\mathrm{s})}: \pm 0.0001$.

$x_{1}: \pm 1.5 \%$ (relative error).

\begin{tabular}{ll}
\hline \hline Components: & Original Measurements: \\
(1) Anthracene; $\mathrm{C}_{14} \mathrm{H}_{10} ;[120-12-7]$ & ${ }^{17} \mathrm{~K}$. J. Pribyla, M. A. Spurgin, I. \\
(2) Cyclohexane; $\mathrm{C}_{6} \mathrm{H}_{12} ;[110-82-7]$ & Chuca, and W. E. Acree, Jr., J. \\
(3) 2-Methoxy-2-methylpropane; & Chem. Eng. Data 45, 530 (2000). \\
$\mathrm{C}_{5} \mathrm{H}_{12} \mathrm{O} ;[1634-04-4]$ & \\
(4) 2-Butanol; $\mathrm{C}_{4} \mathrm{H}_{10} \mathrm{O} ;[78-92-2]$ & \\
\hline Variables: & Prepared by: \\
$T / \mathrm{K}=298.15 ;$ Solvent Composition & W. E. Acree, Jr. \\
\hline
\end{tabular}

Experimental Values

Solubility of anthracene in cyclohexane +2 -methoxy-2-methylpropane +2 butanol mixtures ${ }^{\mathrm{a}}$

\begin{tabular}{lcccc}
\hline \hline$x_{3}(\mathrm{~s})$ & $x_{4}{ }^{(\mathrm{s})}$ & $x_{3}$ & $x_{4}$ & $x_{1}$ \\
\hline 0.0000 & 0.0000 & 0.0000 & 0.0000 & 0.001553 \\
1.0000 & 0.0000 & 0.9969 & 0.0000 & 0.003050 \\
0.0000 & 1.0000 & 0.0000 & 0.9994 & 0.000585 \\
0.2945 & 0.3777 & 0.2939 & 0.3769 & 0.002005 \\
0.1268 & 0.3717 & 0.1267 & 0.3713 & 0.001145 \\
0.2292 & 0.2879 & 0.2287 & 0.2873 & 0.002021 \\
0.2184 & 0.5452 & 0.2180 & 0.5443 & 0.001632 \\
0.6551 & 0.1352 & 0.6533 & 0.1348 & 0.002762 \\
0.6462 & 0.2438 & 0.6445 & 0.2432 & 0.002619 \\
0.1666 & 0.7336 & 0.1664 & 0.7327 & 0.001192 \\
0.0909 & 0.5448 & 0.0908 & 0.5440 & 0.001403 \\
0.3364 & 0.5587 & 0.3358 & 0.5577 & 0.001730 \\
0.4409 & 0.4484 & 0.4400 & 0.4475 & 0.002060 \\
0.1417 & 0.1748 & 0.1414 & 0.1745 & 0.001923 \\
0.6446 & 0.1956 & 0.6429 & 0.1951 & 0.002631 \\
0.4488 & 0.2987 & 0.4478 & 0.2980 & 0.002332 \\
0.0917 & 0.2284 & 0.0915 & 0.2280 & 0.001802 \\
0.0853 & 0.7320 & 0.0852 & 0.7312 & 0.001076 \\
0.1655 & 0.1368 & 0.1652 & 0.1365 & 0.002016 \\
0.0957 & 0.4342 & 0.0956 & 0.4335 & 0.001567 \\
0.3628 & 0.1365 & 0.3619 & 0.1362 & 0.002403 \\
0.4469 & 0.1467 & 0.4458 & 0.1463 & 0.002520 \\
\hline
\end{tabular}

${ }^{\mathrm{a}} x_{3}{ }^{(\mathrm{s})}$ : initial mole fraction of component 3 in the ternary solvent mixture, $x_{4}{ }^{(\mathrm{s})}$ : initial mole fraction of component 4 in the ternary solvent mixture; $x_{1}$ : mole fraction solubility of the solute; $x_{3}$ : mole fraction of component 3 in the quaternary solution; $x_{4}$ : mole fraction of component 4 in the quaternary solution.

\section{Auxiliary Information}

\section{Method/Apparatus/Procedure:}

Constant-temperature bath, calorimetric thermometer, and an ultraviolet/ visible spectrophotometer.

Ternary solvent mixtures were prepared by mass. Excess solute and solvent were placed in amber glass bottles and allowed to equilibrate for several days at constant temperature. Attainment of equilibrium was verified by several repetitive measurements and by approaching equilibrium from supersaturation. Aliquots of saturated solutions were transferred through a coarse filter into volumetric flasks, weighed and diluted with methanol. Molar concentrations were determined by spectrophotometric measurements at $356 \mathrm{~nm}$.

Source and Purity of Chemicals:

(1) $99+\%$, Aldrich Chemical Company, Milwaukee, WI, USA, was used as received.

(2) $99.9+\%$, HPLC grade, Aldrich Chemical Company.

(3) $99.9 \%$, Arco Chemical Company, USA.

(4) $99+\%$, anhydrous, Aldrich Chemical Company.

Components 2-4 were stored over molecular sieves and distilled shortly before use. 
Estimated Error:

Temperature: $\pm 0.1 \mathrm{~K}$

$x_{3}{ }^{(\mathrm{s})}: \pm 0.0001$.

$x_{4}{ }^{(\mathrm{s})}: \pm 0.0001$

$x_{1}: \pm 1.5 \%$ (relative error)

\begin{tabular}{ll}
\hline \hline Components: & Original Measurements: \\
(1) Anthracene; $\mathrm{C}_{14} \mathrm{H}_{10} ;[120-12-7]$ & ${ }^{17} \mathrm{~K}$. J. Pribyla, M. A. Spurgin, I. \\
(2) Cyclohexane; $\mathrm{C}_{6} \mathrm{H}_{12} ;[110-82-7]$ & Chuca, and W. E. Acree, Jr., J. \\
(3) 2-Methoxy-2-methylpropane; & Chem. Eng. Data 45, 530 (2000). \\
$\mathrm{C}_{5} \mathrm{H}_{12} \mathrm{O} ;[$ [1634-04-4] & \\
(4) 2-Methyl-1-propanol; $\mathrm{C}_{4} \mathrm{H}_{10} \mathrm{O} ;$ & \\
[78-83-1] & \\
\hline Variables: & Prepared by: \\
$T / \mathrm{K}=298.15 ;$ Solvent Composition & W. E. Acree, Jr. \\
\hline
\end{tabular}

Experimental Values

Solubility of anthracene in cyclohexane +2 -methoxy-2-methylpropane +2 methyl-1-propanol mixtures ${ }^{\mathrm{a}}$

\begin{tabular}{|c|c|c|c|c|}
\hline$x_{3}{ }^{(\mathrm{s})}$ & $x_{4}{ }^{(\mathrm{s})}$ & $x_{3}$ & $x_{4}$ & $x_{1}$ \\
\hline 0.0000 & 0.0000 & 0.0000 & 0.0000 & 0.001553 \\
\hline 1.0000 & 0.0000 & 0.9969 & 0.0000 & 0.003050 \\
\hline 0.0000 & 1.0000 & 0.0000 & 0.9995 & 0.000470 \\
\hline 0.2866 & 0.3853 & 0.2861 & 0.3846 & 0.001806 \\
\hline 0.1285 & 0.7310 & 0.1284 & 0.7303 & 0.000984 \\
\hline 0.2268 & 0.2948 & 0.2264 & 0.2943 & 0.001863 \\
\hline 0.2201 & 0.5440 & 0.2198 & 0.5432 & 0.001429 \\
\hline 0.6512 & 0.1402 & 0.6475 & 0.1398 & 0.002672 \\
\hline 0.4803 & 0.4359 & 0.4794 & 0.4350 & 0.001960 \\
\hline 0.1668 & 0.7338 & 0.1666 & 0.7330 & 0.001030 \\
\hline 0.0977 & 0.5295 & 0.0976 & 0.5288 & 0.001265 \\
\hline 0.3440 & 0.5467 & 0.3434 & 0.5458 & 0.001575 \\
\hline 0.4361 & 0.4523 & 0.4353 & 0.4515 & 0.001871 \\
\hline 0.1388 & 0.1978 & 0.1385 & 0.1974 & 0.001824 \\
\hline 0.6134 & 0.1835 & 0.6118 & 0.1830 & 0.002560 \\
\hline 0.4470 & 0.3010 & 0.4460 & 0.3003 & 0.002166 \\
\hline 0.1026 & 0.2305 & 0.1024 & 0.2301 & 0.001714 \\
\hline 0.0936 & 0.7256 & 0.0935 & 0.7249 & 0.000944 \\
\hline 0.1453 & 0.1375 & 0.1450 & 0.1372 & 0.001868 \\
\hline 0.0978 & 0.4371 & 0.0977 & 0.4364 & 0.001426 \\
\hline 0.3661 & 0.1325 & 0.3653 & 0.1322 & 0.002275 \\
\hline 0.3237 & 0.4255 & 0.3231 & 0.4247 & 0.001786 \\
\hline
\end{tabular}

\section{Auxiliary Information}

\section{Method/Apparatus/Procedure:}

Constant-temperature bath, calorimetric thermometer, and an ultraviolet/ visible spectrophotometer.

Ternary solvent mixtures were prepared by mass. Excess solute and solvent were placed in amber glass bottles and allowed to equilibrate for several days at constant temperature. Attainment of equilibrium was verified by several repetitive measurements and by approaching equilibrium from supersaturation. Aliquots of saturated solutions were transferred through a coarse filter into volumetric flasks, weighed and diluted with methanol. Molar concentrations were determined by spectrophotometric measurements at $356 \mathrm{~nm}$.
Source and Purity of Chemicals:

(1) $99+\%$, Aldrich Chemical Company, Milwaukee, WI, USA, was used as received.

(2) $99.9+\%$, HPLC grade, Aldrich Chemical Company.

(3) $99.9 \%$, Arco Chemical Company, USA.

(4) $99+\%$, anhydrous, Aldrich Chemical Company.

Components 2-4 were stored over molecular sieves and distilled shortly before use.

\section{Estimated Error:}

Temperature: $\pm 0.1 \mathrm{~K}$.

$x_{3}{ }^{(\mathrm{s})}: \pm 0.0001$.

$x_{4}{ }^{(\mathrm{s})}: \pm 0.0001$.

$x_{1}: \pm 1.5 \%$ (relative error).

\begin{tabular}{|c|c|}
\hline $\begin{array}{l}\text { Components: } \\
\text { (1) Anthracene; } \mathrm{C}_{14} \mathrm{H}_{10} ;[120-12-7] \\
\text { (2) 2,2,4-Trimethylpentane; } \mathrm{C}_{8} \mathrm{H}_{18} \text {; } \\
\text { [540-84-1] } \\
\text { (3) 2-Methoxy-2-methylpropane; } \\
\mathrm{C}_{5} \mathrm{H}_{12} \mathrm{O} ;[1634-04-4] \\
\text { (4) 1-Propanol; } \mathrm{C}_{3} \mathrm{H}_{8} \mathrm{O} ;[71-23-8]\end{array}$ & $\begin{array}{l}\text { Original Measurements: } \\
{ }^{15} \text { K. J. Pribyla, C. Ezell, T. T. Van, } \\
\text { and W. E. Acree, Jr., J. Chem. } \\
\text { Eng. Data 45, } 974 \text { (2000). }\end{array}$ \\
\hline $\begin{array}{l}\text { Variables: } \\
T / \mathrm{K}=298.15 \text {; Solvent Composition }\end{array}$ & $\begin{array}{l}\text { Prepared by: } \\
\text { W. E. Acree, Jr. }\end{array}$ \\
\hline
\end{tabular}

Solubility of anthracene in 2,2,4-trimethylpentane + 2-methoxy-2-methylpropane +1 -propanol mixtures ${ }^{\mathrm{a}}$

\begin{tabular}{|c|c|c|c|c|}
\hline$x_{3}{ }^{(\mathrm{s})}$ & $x_{4}^{(s)}$ & $x_{3}$ & $x_{4}$ & $x_{1}$ \\
\hline 0.0000 & 0.0000 & 0.0000 & 0.0000 & 0.001074 \\
\hline 1.0000 & 0.0000 & 0.9969 & 0.0000 & 0.003050 \\
\hline 0.0000 & 1.0000 & 0.0000 & 0.9994 & 0.000591 \\
\hline 0.3022 & 0.4800 & 0.3017 & 0.4792 & 0.001600 \\
\hline 0.1115 & 0.8065 & 0.1114 & 0.8057 & 0.000979 \\
\hline 0.2499 & 0.4044 & 0.2495 & 0.4038 & 0.001488 \\
\hline 0.2072 & 0.6457 & 0.2069 & 0.6449 & 0.001276 \\
\hline 0.6787 & 0.1790 & 0.6770 & 0.1786 & 0.002437 \\
\hline 0.6282 & 0.2999 & 0.6267 & 0.2992 & 0.002348 \\
\hline 0.1462 & 0.7985 & 0.1461 & 0.7977 & 0.001023 \\
\hline 0.0925 & 0.6599 & 0.0924 & 0.6591 & 0.001038 \\
\hline 0.3160 & 0.6206 & 0.3155 & 0.6196 & 0.001537 \\
\hline 0.4845 & 0.4368 & 0.4835 & 0.4359 & 0.001987 \\
\hline 0.1683 & 0.2757 & 0.1681 & 0.2753 & 0.001360 \\
\hline 0.6553 & 0.2404 & 0.6537 & 0.2398 & 0.002409 \\
\hline 0.4532 & 0.3780 & 0.4523 & 0.3773 & 0.001930 \\
\hline 0.1153 & 0.3511 & 0.1152 & 0.3507 & 0.001242 \\
\hline 0.0777 & 0.8149 & 0.0776 & 0.8142 & 0.000890 \\
\hline 0.2289 & 0.2005 & 0.2285 & 0.2002 & 0.001464 \\
\hline 0.0987 & 0.5773 & 0.0986 & 0.5767 & 0.001102 \\
\hline 0.4327 & 0.1895 & 0.4319 & 0.1892 & 0.001839 \\
\hline 0.5133 & 0.1858 & 0.5122 & 0.1854 & 0.002064 \\
\hline
\end{tabular}

${ }^{\mathrm{a}} x_{3}{ }^{(\mathrm{s})}$ : initial mole fraction of component 3 in the ternary solvent mixture, $x_{4}{ }^{(\mathrm{s})}$ : initial mole fraction of component 4 in the ternary solvent mixture; $x_{1}$ : mole fraction solubility of the solute; $x_{3}$ : mole fraction of component 3 in the quaternary solution; $x_{4}$ : mole fraction of component 4 in the quaternary solution. 


\section{Auxiliary Information}

Method/Apparatus/Procedure:

Constant-temperature bath, calorimetric thermometer, and an ultraviolet/ visible spectrophotometer.

Ternary solvent mixtures were prepared by mass. Excess solute and solvent were placed in amber glass bottles and allowed to equilibrate for several days at constant temperature. Attainment of equilibrium was verified by several repetitive measurements and by approaching equilibrium from supersaturation. Aliquots of saturated solutions were transferred through a coarse filter into volumetric flasks, weighed and diluted with methanol. Molar concentrations were determined by spectrophotometric measurements at $356 \mathrm{~nm}$.

\section{Source and Purity of Chemicals:}

(1) $99.9+\%$, Aldrich Chemical Company, Milwaukee, WI, USA, was used as received.

(2) $99.7 \%$, HPLC grade, Aldrich Chemical Company.

(3) $99.9 \%$, Arco Chemical Company, USA.

(4) $99+\%$, anhydrous, Aldrich Chemical Company.

Components 2-4 were stored over molecular sieves and distilled shortly before use.

\section{Estimated Error:}

Temperature: $\pm 0.1 \mathrm{~K}$.

$x_{3}{ }^{(\mathrm{s})}: \pm 0.0001$.

$x_{4}{ }^{(\mathrm{s})}: \pm 0.0001$.

$x_{1}: \pm 1.5 \%$ (relative error).

\section{Components:}

(1) Anthracene; $\mathrm{C}_{14} \mathrm{H}_{10}$; [120-12-7]

(2) 2,2,4-Trimethylpentane; $\mathrm{C}_{8} \mathrm{H}_{18}$;

[540-84-1]

(3) 2-Methoxy-2-methylpropane;

$\mathrm{C}_{5} \mathrm{H}_{12} \mathrm{O}$; [1634-04-4]

(4) 2-Propanol; $\mathrm{C}_{3} \mathrm{H}_{8} \mathrm{O}$; [67-63-0]

\begin{tabular}{ll}
\hline Variables: & Prepared by: \\
$T / \mathrm{K}=298.15 ;$ Solvent Composition & W. E. Acree, Jr. \\
\hline
\end{tabular}

\section{Experimental Values}

Solubility of anthracene in 2,2,4-trimethylpentane + 2-methoxy-2-methylpropane +2 -propanol mixtures ${ }^{\mathrm{a}}$

\begin{tabular}{lcccc}
\hline \hline$x_{3}{ }^{(\mathrm{s})}$ & $x_{4}{ }^{(\mathrm{s})}$ & $x_{3}$ & $x_{4}$ & $x_{1}$ \\
\hline 0.0000 & 0.0000 & 0.0000 & 0.0000 & 0.001074 \\
1.0000 & 0.0000 & 0.9969 & 0.0000 & 0.003050 \\
0.0000 & 1.0000 & 0.0000 & 0.9996 & 0.000411 \\
0.3025 & 0.4726 & 0.3021 & 0.4719 & 0.001460 \\
0.1206 & 0.7908 & 0.1205 & 0.7902 & 0.000804 \\
0.2525 & 0.3923 & 0.2521 & 0.3918 & 0.001376 \\
0.2070 & 0.6393 & 0.2068 & 0.6386 & 0.001109 \\
0.6818 & 0.1686 & 0.6802 & 0.1682 & 0.002368 \\
0.6307 & 0.2938 & 0.6293 & 0.2931 & 0.002241 \\
0.1496 & 0.7893 & 0.1495 & 0.7886 & 0.000834 \\
0.0976 & 0.6857 & 0.0975 & 0.6851 & 0.000913 \\
0.3152 & 0.6239 & 0.3148 & 0.6231 & 0.001342 \\
0.4167 & 0.5169 & 0.4160 & 0.5161 & 0.001631
\end{tabular}

$\begin{array}{lllll}0.1770 & 0.2681 & 0.1768 & 0.2677 & 0.001322 \\ 0.6584 & 0.2294 & 0.6569 & 0.2289 & 0.002338 \\ 0.4583 & 0.3673 & 0.4575 & 0.3666 & 0.001810 \\ 0.1127 & 0.3450 & 0.1126 & 0.3446 & 0.001165 \\ 0.0794 & 0.8074 & 0.0793 & 0.8068 & 0.000724 \\ 0.2333 & 0.1945 & 0.2330 & 0.1942 & 0.001405 \\ 0.1051 & 0.5692 & 0.1050 & 0.5686 & 0.001011 \\ 0.4251 & 0.1994 & 0.4243 & 0.1990 & 0.001806 \\ 0.5172 & 0.1817 & 0.5162 & 0.1813 & 0.001986\end{array}$

${ }^{\mathrm{a}} x_{3}{ }^{(\mathrm{s})}$ : initial mole fraction of component 3 in the ternary solvent mixture, $x_{4}{ }^{(\mathrm{s})}$ : initial mole fraction of component 4 in the ternary solvent mixture; $x_{1}$ : mole fraction solubility of the solute; $x_{3}$ : mole fraction of component 3 in the quaternary solution; $x_{4}$ : mole fraction of component 4 in the quaternary solution.

\section{Auxiliary Information}

\section{Method/Apparatus/Procedure:}

Constant-temperature bath, calorimetric thermometer, and an ultraviolet/ visible spectrophotometer.

Ternary solvent mixtures were prepared by mass. Excess solute and solvent were placed in amber glass bottles and allowed to equilibrate for several days at constant temperature. Attainment of equilibrium was verified by several repetitive measurements and by approaching equilibrium from supersaturation. Aliquots of saturated solutions were transferred through a coarse filter into volumetric flasks, weighed and diluted with methanol. Molar concentrations were determined by spectrophotometric measurements at $356 \mathrm{~nm}$.

\section{Source and Purity of Chemicals:}

(1) $99+\%$, Aldrich Chemical Company, Milwaukee, WI, USA, was used as received.

(2) $99.7 \%$, HPLC grade, Aldrich Chemical Company.

(3) $99.9 \%$, Arco Chemical Company, USA.

(4) $99+\%$, anhydrous, Aldrich Chemical Company.

Components 2-4 were stored over molecular sieves and distilled shortly before use.

\section{Estimated Error:}

Temperature: $\pm 0.1 \mathrm{~K}$.

$x_{3}{ }^{(\mathrm{s})}: \pm 0.0001$.

$x_{4}{ }^{(\mathrm{s})}: \pm 0.0001$.

$x_{1}: \pm 1.5 \%$ (relative error)

\begin{tabular}{|c|c|}
\hline $\begin{array}{l}\text { Components: } \\
\text { (1) Anthracene; } \mathrm{C}_{14} \mathrm{H}_{10} ;[120-12-7] \\
\text { (2) } 2,2,4 \text {-Trimethylpentane; } \mathrm{C}_{8} \mathrm{H}_{18} \text {; } \\
\text { [540-84-1] } \\
\text { (3) 2-Methoxy-2-methylpropane; } \\
\mathrm{C}_{5} \mathrm{H}_{12} \mathrm{O} ;[1634-04-4] \\
\text { (4) } 1 \text {-Butanol; } \mathrm{C}_{4} \mathrm{H}_{10} \mathrm{O} ;[71-36-3]\end{array}$ & $\begin{array}{l}\text { Original Measurements: } \\
{ }^{15} \text { K. J. Pribyla, C. Ezell, T. T. Van, } \\
\text { and W. E. Acree, Jr., J. Chem. } \\
\text { Eng. Data 45, } 974 \text { (2000). }\end{array}$ \\
\hline $\begin{array}{l}\text { Variables: } \\
T / \mathrm{K}=298.15 \text {; Solvent Composition }\end{array}$ & $\begin{array}{l}\text { Prepared by: } \\
\text { W. E. Acree, Jr. }\end{array}$ \\
\hline
\end{tabular}




\section{Experimental Values}

Solubility of anthracene in 2,2,4-trimethylpentane + 2-methoxy-2-methylpropane +1 -butanol mixtures ${ }^{\mathrm{a}}$

\begin{tabular}{lcccc}
\hline \hline$x_{3}{ }^{(\mathrm{s})}$ & $x_{4}{ }^{(\mathrm{s})}$ & $x_{3}$ & $x_{4}$ & $x_{1}$ \\
\hline 0.0000 & 0.0000 & 0.0000 & 0.0000 & 0.001074 \\
1.0000 & 0.0000 & 0.9969 & 0.0000 & 0.003050 \\
0.0000 & 1.0000 & 0.0000 & 0.9992 & 0.000801 \\
0.3297 & 0.4312 & 0.3291 & 0.4305 & 0.001732 \\
0.1350 & 0.7684 & 0.1348 & 0.7675 & 0.001194 \\
0.2702 & 0.3551 & 0.2698 & 0.3545 & 0.001591 \\
0.2799 & 0.5637 & 0.2794 & 0.5628 & 0.001543 \\
0.7041 & 0.1461 & 0.7023 & 0.1457 & 0.002518 \\
0.6701 & 0.2538 & 0.6684 & 0.2531 & 0.002571 \\
0.1717 & 0.7595 & 0.1715 & 0.7585 & 0.001302 \\
0.1023 & 0.6194 & 0.1022 & 0.6186 & 0.001223 \\
0.3537 & 0.5745 & 0.3531 & 0.5735 & 0.001791 \\
0.4492 & 0.4738 & 0.4483 & 0.4728 & 0.002040 \\
0.1847 & 0.2425 & 0.1844 & 0.2422 & 0.001402 \\
0.6817 & 0.2069 & 0.6800 & 0.2064 & 0.002490 \\
0.4894 & 0.3289 & 0.4884 & 0.3282 & 0.001993 \\
0.1286 & 0.3028 & 0.1284 & 0.3024 & 0.001332 \\
0.0935 & 0.7783 & 0.0934 & 0.7774 & 0.001105 \\
0.2380 & 0.1708 & 0.2376 & 0.1705 & 0.001473 \\
0.1111 & 0.5273 & 0.1110 & 0.5266 & 0.001279 \\
0.4425 & 0.1612 & 0.4417 & 0.1609 & 0.001862 \\
0.5261 & 0.1594 & 0.5250 & 0.1591 & 0.002062 \\
\hline$x_{3}(5): 1 n i a r y$
\end{tabular}

${ }^{\mathrm{a}} x_{3}{ }^{(\mathrm{s})}$ : initial mole fraction of component 3 in the ternary solvent mixture, $x_{4}{ }^{(\mathrm{s})}$. initial mole fraction of component 4 in the ternary solvent mixture; $x_{1}$ : mole fraction solubility of the solute; $x_{3}$ : mole fraction of component 3 in the quaternary solution; $x_{4}$ : mole fraction of component 4 in the quaternary solution.

\section{Auxiliary Information}

\section{Method/Apparatus/Procedure:}

Constant-temperature bath, calorimetric thermometer, and an ultraviolet/ visible spectrophotometer.

Ternary solvent mixtures were prepared by mass. Excess solute and solvent were placed in amber glass bottles and allowed to equilibrate for several days at constant temperature. Attainment of equilibrium was verified by several repetitive measurements and by approaching equilibrium from supersaturation. Aliquots of saturated solutions were transferred through a coarse filter into volumetric flasks, weighed and diluted with methanol. Molar concentrations were determined by spectrophotometric measurements at $356 \mathrm{~nm}$.

\section{Source and Purity of Chemicals:}

(1) $99+\%$, Aldrich Chemical Company, Milwaukee, WI, USA, was used as received.

(2) $99.7 \%$, HPLC grade, Aldrich Chemical Company, Milwaukee, WI, USA. (3) $99.9 \%$, Arco Chemical Company, USA.

(4) $99.8+\%$, HPLC grade, Aldrich Chemical Company.

Components 2-4 were stored over molecular sieves and distilled shortly before use.

\section{Estimated Error:}

Temperature: $\pm 0.1 \mathrm{~K}$.

$x_{3}^{(\mathrm{s})}: \pm 0.0001$.

$x_{4}^{(\mathrm{s})}: \pm 0.0001$

$x_{1}: \pm 1.5 \%$ (relative error)

\section{Components:}

(1) Anthracene; $\mathrm{C}_{14} \mathrm{H}_{10} ;[120-12-7]$

(2) 2,2,4-Trimethylpentane; $\mathrm{C}_{8} \mathrm{H}_{18}$; [540-84-1]

(3) 2-Methoxy-2-methylpropane; $\mathrm{C}_{5} \mathrm{H}_{12} \mathrm{O}$; [1634-04-4]

(4) 2-Butanol; $\mathrm{C}_{4} \mathrm{H}_{10} \mathrm{O}$; [78-92-2]

\begin{tabular}{ll}
\hline Variables: & Prepared by: \\
$T / \mathrm{K}=298.15$; Solvent Composition & W. E. Acree, Jr. \\
\hline
\end{tabular}

Experimental Values

Solubility of anthracene in 2,2,4-trimethylpentane + 2-methoxy-2-methylpropane +2 -butanol mixtures ${ }^{\mathrm{a}}$

\begin{tabular}{|c|c|c|c|c|}
\hline$x_{3}{ }^{(s)}$ & $x_{4}{ }^{(\mathrm{s})}$ & $x_{3}$ & $x_{4}$ & $x_{1}$ \\
\hline 0.0000 & 0.0000 & 0.0000 & 0.0000 & 0.001074 \\
\hline 1.0000 & 0.0000 & 0.9969 & 0.0000 & 0.003050 \\
\hline 0.0000 & 1.0000 & 0.0000 & 0.9994 & 0.000585 \\
\hline 0.3282 & 0.4312 & 0.3277 & 0.4305 & 0.001618 \\
\hline 0.1349 & 0.7721 & 0.1348 & 0.7713 & 0.001025 \\
\hline 0.2709 & 0.3482 & 0.2705 & 0.3477 & 0.001528 \\
\hline 0.2341 & 0.5960 & 0.2338 & 0.5952 & 0.001357 \\
\hline 0.7080 & 0.1379 & 0.7063 & 0.1376 & 0.002437 \\
\hline 0.6683 & 0.2531 & 0.6667 & 0.2525 & 0.002394 \\
\hline 0.1919 & 0.7358 & 0.1917 & 0.7349 & 0.001168 \\
\hline 0.1111 & 0.6038 & 0.1110 & 0.6031 & 0.001114 \\
\hline 0.3550 & 0.5714 & 0.3544 & 0.5705 & 0.001662 \\
\hline 0.4500 & 0.4744 & 0.4491 & 0.4735 & 0.001924 \\
\hline 0.1751 & 0.2358 & 0.1749 & 0.2355 & 0.001377 \\
\hline 0.6829 & 0.2037 & 0.6813 & 0.2032 & 0.002384 \\
\hline 0.4879 & 0.3308 & 0.4870 & 0.3302 & 0.001945 \\
\hline 0.1069 & 0.3059 & 0.1068 & 0.3055 & 0.001267 \\
\hline 0.0923 & 0.7822 & 0.0922 & 0.7815 & 0.000947 \\
\hline 0.4887 & 0.1040 & 0.4878 & 0.1038 & 0.001881 \\
\hline 0.1066 & 0.5314 & 0.1065 & 0.5308 & 0.001150 \\
\hline 0.4415 & 0.1627 & 0.4407 & 0.1624 & 0.001854 \\
\hline 0.5336 & 0.1569 & 0.5325 & 0.1566 & 0.002052 \\
\hline
\end{tabular}

${ }^{\mathrm{a}} x_{3}{ }^{(\mathrm{s})}$ : initial mole fraction of component 3 in the ternary solvent mixture, $x_{4}{ }^{(\mathrm{s})}$ : initial mole fraction of component 4 in the ternary solvent mixture; $x_{1}$ : mole fraction solubility of the solute; $x_{3}$ : mole fraction of component 3 in the quaternary solution; $x_{4}$ : mole fraction of component 4 in the quaternary solution.

\section{Auxiliary Information}

\section{Method/Apparatus/Procedure:}

Constant-temperature bath, calorimetric thermometer, and an ultraviolet/ visible spectrophotometer.

Ternary solvent mixtures were prepared by mass. Excess solute and solvent were placed in amber glass bottles and allowed to equilibrate for several days at constant temperature. Attainment of equilibrium was verified by several repetitive measurements and by approaching equilibrium from supersaturation. Aliquots of saturated solutions were transferred through a coarse filter into volumetric flasks, weighed and diluted with methanol. Molar concentrations were determined by spectrophotometric measurements at $356 \mathrm{~nm}$.

\section{Source and Purity of Chemicals:}

(1) $99+\%$, Aldrich Chemical Company, Milwaukee, WI, USA, was used as received.

(2) $99.7 \%$, HPLC grade, Aldrich Chemical Company.

(3) $99.9 \%$, Arco Chemical Company, USA.

(4) $99+\%$, anhydrous, Aldrich Chemical Company.

Components 2-4 were stored over molecular sieves and distilled shortly before use. 
Estimated Error:

Temperature: $\pm 0.1 \mathrm{~K}$.

$x_{3}{ }^{(\mathrm{s})}: \pm 0.0001$.

$x_{4}{ }^{(\mathrm{s})}: \pm 0.0001$.

$x_{1}: \pm 1.5 \%$ (relative error).

\begin{tabular}{|c|c|}
\hline $\begin{array}{l}\text { Components: } \\
\text { (1) Anthracene; } \mathrm{C}_{14} \mathrm{H}_{10} ;[120-12-7] \\
\text { (2) 2,2,4-Trimethylpentane; } \mathrm{C}_{8} \mathrm{H}_{18} \text {; } \\
\text { [540-84-1] } \\
\text { (3) 2-Methoxy-2-methylpropane; } \\
\mathrm{C}_{5} \mathrm{H}_{12} \mathrm{O} \text {; [1634-04-4] } \\
\text { (4) 2-Methyl-1-propanol; } \mathrm{C}_{4} \mathrm{H}_{10} \mathrm{O} \text {; } \\
\text { [78-83-1] }\end{array}$ & $\begin{array}{l}\text { Original Measurements: } \\
{ }^{15} \text { K. J. Pribyla, C. Ezell, T. T. Van, } \\
\text { and W. E. Acree, Jr., J. Chem. } \\
\text { Eng. Data 45, } 974 \text { (2000). }\end{array}$ \\
\hline $\begin{array}{l}\text { Variables: } \\
T / \mathrm{K}=298.15 \text {; Solvent Composition }\end{array}$ & $\begin{array}{l}\text { Prepared by: } \\
\text { W. E. Acree, Jr. }\end{array}$ \\
\hline
\end{tabular}

Experimental Values

Solubility of anthracene in 2,2,4-trimethylpentane +2 -methoxy-2-methylpropane +2 -methyl-1-propanol mixtures ${ }^{\mathrm{a}}$

\begin{tabular}{lcccc}
\hline \hline$x_{3}{ }^{(\mathrm{s})}$ & $x_{4}{ }^{(\mathrm{s})}$ & $x_{3}$ & $x_{4}$ & $x_{1}$ \\
\hline 0.0000 & 0.0000 & 0.0000 & 0.0000 & 0.001074 \\
1.0000 & 0.0000 & 0.9969 & 0.0000 & 0.003050 \\
0.0000 & 1.0000 & 0.0000 & 0.9995 & 0.000470 \\
0.3310 & 0.4287 & 0.3305 & 0.4281 & 0.001505 \\
0.1367 & 0.7633 & 0.1366 & 0.7626 & 0.000877 \\
0.2781 & 0.3455 & 0.2777 & 0.3450 & 0.001451 \\
0.2387 & 0.5900 & 0.2384 & 0.5893 & 0.001200 \\
0.7001 & 0.1460 & 0.6985 & 0.1457 & 0.002284 \\
0.6674 & 0.2535 & 0.6659 & 0.2529 & 0.002302 \\
0.1780 & 0.7542 & 0.1778 & 0.7535 & 0.000946 \\
0.1080 & 0.6133 & 0.1079 & 0.6127 & 0.001005 \\
0.3615 & 0.5652 & 0.3610 & 0.5644 & 0.001457 \\
0.4562 & 0.4708 & 0.4554 & 0.4700 & 0.001699 \\
0.1858 & 0.2349 & 0.1856 & 0.2346 & 0.001328 \\
0.6859 & 0.1989 & 0.6843 & 0.1984 & 0.002328 \\
0.4993 & 0.3194 & 0.4984 & 0.3188 & 0.001877 \\
0.1303 & 0.3031 & 0.1301 & 0.3027 & 0.001207 \\
0.0957 & 0.7748 & 0.0956 & 0.7742 & 0.000819 \\
0.2420 & 0.1668 & 0.2417 & 0.1666 & 0.001422 \\
0.1158 & 0.5180 & 0.1157 & 0.5174 & 0.001068 \\
0.4469 & 0.1594 & 0.4461 & 0.1591 & 0.001794 \\
0.5327 & 0.1546 & 0.5317 & 0.1542 & 0.001963 \\
\hline$x_{3}(5)$ & &
\end{tabular}

${ }^{\mathrm{a}} x_{3}{ }^{(\mathrm{s})}$ : initial mole fraction of component 3 in the ternary solvent mixture, $x_{4}{ }^{(\mathrm{s})}$ : initial mole fraction of component 4 in the ternary solvent mixture; $x_{1}$ : mole fraction solubility of the solute; $x_{3}$ : mole fraction of component 3 in the quaternary solution; $x_{4}$ : mole fraction of component 4 in the quaternary solution.

\section{Auxiliary Information}

\section{Method/Apparatus/Procedure:}

Constant-temperature bath, calorimetric thermometer, and an ultraviolet/ visible spectrophotometer.

Ternary solvent mixtures were prepared by mass. Excess solute and solvent were placed in amber glass bottles and allowed to equilibrate for several days at constant temperature. Attainment of equilibrium was verified by several repetitive measurements and by approaching equilibrium from

supersaturation. Aliquots of saturated solutions were transferred through a coarse filter into volumetric flasks, weighed and diluted with methanol. Molar concentrations were determined by spectrophotometric measurements at $356 \mathrm{~nm}$.
Source and Purity of Chemicals:

(1) $99+\%$, Aldrich Chemical Company, Milwaukee, WI, USA, was used as received.

(2) $99.7 \%$, HPLC grade, Aldrich Chemical Company.

(3) $99.9 \%$, Arco Chemical Company, USA.

(4) $99+\%$, anhydrous, Aldrich Chemical Company.

Components 2-4 were stored over molecular sieves and distilled shortly before use.

Estimated Error:

Temperature: $\pm 0.1 \mathrm{~K}$.

$x_{3}{ }^{(s)}: \pm 0.0001$.

$x_{4}{ }^{(\mathrm{s})}: \pm 0.0001$.

$x_{1}: \pm 1.5 \%$ (relative error)

\subsection{Anthracene solubility data in ternary alkane + 1,4-dioxane + alcohol solvent mixtures}

\begin{tabular}{ll}
\hline \hline Components: & Original Measurements: \\
(1) Anthracene; $\mathrm{C}_{14} \mathrm{H}_{10} ;[120-12-7]$ & ${ }^{13}$ K. J. Pribyla, M. A. Spurgin, I. \\
(2) Heptane; $\mathrm{C}_{7} \mathrm{H}_{16} ;[142-82-5]$ & Chuca, and W. E. Acree, Jr., J. \\
(3) 1,4-Dioxane; $\mathrm{C}_{4} \mathrm{H}_{8} \mathrm{O}_{2} ;[123-91-1]$ & Chem. Eng. Data 45, 965 (2000). \\
(4) 1-Propanol; $\mathrm{C}_{3} \mathrm{H}_{8} \mathrm{O} ;[71-23-8]$ & \\
\hline Variables: & Prepared by: \\
$T / \mathrm{K}=298.15 ;$ Solvent Composition & W. E. Acree, Jr. \\
\hline
\end{tabular}

Experimental Values

Solubility of anthracene in heptane +1 ,4-dioxane +1 -propanol mixtures ${ }^{\mathrm{a}}$

\begin{tabular}{lcccc}
\hline \hline$x_{3}{ }^{(\mathrm{s})}$ & $x_{4}{ }^{(\mathrm{s})}$ & $x_{3}$ & $x_{4}$ & $x_{1}$ \\
\hline 0.0000 & 0.0000 & 0.0000 & 0.0000 & 0.001571 \\
1.0000 & 0.0000 & 0.9917 & 0.0000 & 0.008329 \\
0.0000 & 1.0000 & 0.0000 & 0.9994 & 0.000591 \\
0.3712 & 0.4165 & 0.3698 & 0.4150 & 0.003703 \\
0.1536 & 0.7590 & 0.1534 & 0.7578 & 0.001606 \\
0.3074 & 0.3502 & 0.3069 & 0.3490 & 0.003491 \\
0.2641 & 0.5860 & 0.2634 & 0.5845 & 0.002585 \\
0.7397 & 0.1349 & 0.7345 & 0.1340 & 0.007021 \\
0.6998 & 0.2406 & 0.6956 & 0.2392 & 0.006017 \\
0.1930 & 0.7471 & 0.1926 & 0.7457 & 0.001822 \\
0.1225 & 0.6222 & 0.1223 & 0.6121 & 0.001786 \\
0.3907 & 0.5470 & 0.3894 & 0.5452 & 0.003299 \\
0.4856 & 0.4491 & 0.4836 & 0.4473 & 0.004110 \\
0.2154 & 0.2433 & 0.2148 & 0.2426 & 0.002909 \\
0.7117 & 0.1964 & 0.7072 & 0.1951 & 0.006375 \\
0.5300 & 0.3409 & 0.5274 & 0.3392 & 0.004906 \\
0.1528 & 0.3148 & 0.1524 & 0.3140 & 0.002429 \\
0.1116 & 0.7763 & 0.1114 & 0.7752 & 0.001409 \\
0.2817 & 0.1617 & 0.2807 & 0.1611 & 0.003554 \\
0.1363 & 0.5273 & 0.1360 & 0.5262 & 0.002020 \\
0.4946 & 0.1541 & 0.4920 & 0.1533 & 0.005229 \\
0.5777 & 0.1538 & 0.5743 & 0.1529 & 0.005803 \\
\hline$x_{3}: 5$ & & &
\end{tabular}

${ }^{\mathrm{a}} x_{3}{ }^{(\mathrm{s})}$ : initial mole fraction of component 3 in the ternary solvent mixture, $x_{4}{ }^{(\mathrm{s})}$ : initial mole fraction of component 4 in the ternary solvent mixture; $x_{1}$ : mole fraction solubility of the solute; $x_{3}$ : mole fraction of component 3 in the quaternary solution; $x_{4}$ : mole fraction of component 4 in the quaternary solution. 


\section{Auxiliary Information}

\section{Method/Apparatus/Procedure:}

Constant-temperature bath, calorimetric thermometer, and an ultraviolet/ visible spectrophotometer.

Ternary solvent mixtures were prepared by mass. Excess solute and solvent were placed in amber glass bottles and allowed to equilibrate for several days at constant temperature. Attainment of equilibrium was verified by several repetitive measurements and by approaching equilibrium from supersaturation. Aliquots of saturated solutions were transferred through a coarse filter into volumetric flasks, weighed and diluted with methanol. Molar concentrations were determined by spectrophotometric measurements at $356 \mathrm{~nm}$.

\section{Source and Purity of Chemicals:}

(1) $99+\%$, Aldrich Chemical Company, Milwaukee, WI, USA, was used as received.

(2) $99.9+\%$, HPLC grade, Aldrich Chemical Company.

(3) $99.8 \%$, anhydrous, Aldrich Chemical Company.

(4) $99+\%$, anhydrous, Aldrich Chemical Company.

Components $2-4$ were stored over molecular sieves and distilled shortly before use.

\section{Estimated Error:}

Temperature: $\pm 0.1 \mathrm{~K}$

$x_{3}{ }^{(\mathrm{s})}: \pm 0.0001$.

$x_{4}{ }^{(\mathrm{s})}: \pm 0.0001$

$x_{1}: \pm 1.5 \%$ (relative error).

\section{Components:}

(1) Anthracene; $\mathrm{C}_{14} \mathrm{H}_{10} ;[120-12-7]$

(2) Heptane; $\mathrm{C}_{7} \mathrm{H}_{16} ;[142-82-5]$

(3) 1,4-Dioxane; $\mathrm{C}_{4} \mathrm{H}_{8} \mathrm{O}_{2} ;[123-91-1]$

(4) 2-Propanol; $\mathrm{C}_{3} \mathrm{H}_{8} \mathrm{O}$; [67-63-0]

\begin{tabular}{ll}
\hline Variables: & Prepared by: \\
$T / \mathrm{K}=298.15 ;$ Solvent Composition & W. E. Acree, Jr. \\
\hline
\end{tabular}

\section{Experimental Values}

Solubility of anthracene in heptane +1 ,4-dioxane +2 -propanol mixtures ${ }^{\mathrm{a}}$

\begin{tabular}{lcccc}
\hline \hline$x_{3}{ }^{(\mathrm{s})}$ & $x_{4}{ }^{(\mathrm{s})}$ & $x_{3}$ & $x_{4}$ & $x_{1}$ \\
\hline 0.0000 & 0.0000 & 0.0000 & 0.0000 & 0.001571 \\
1.0000 & 0.0000 & 0.9917 & 0.0000 & 0.008329 \\
0.0000 & 1.0000 & 0.0000 & 0.9996 & 0.000411 \\
0.3675 & 0.4154 & 0.3662 & 0.4139 & 0.003551 \\
0.1522 & 0.7588 & 0.1520 & 0.7577 & 0.001408 \\
0.3065 & 0.3374 & 0.3055 & 0.3363 & 0.003358 \\
0.2740 & 0.5728 & 0.2733 & 0.5714 & 0.002476 \\
0.7351 & 0.1327 & 0.7302 & 0.1318 & 0.006720 \\
0.7021 & 0.2307 & 0.6979 & 0.2393 & 0.005916 \\
0.2017 & 0.7363 & 0.2014 & 0.7351 & 0.001642 \\
0.1216 & 0.6242 & 0.1214 & 0.6232 & 0.001593 \\
0.3939 & 0.5433 & 0.3927 & 0.5416 & 0.003137 \\
0.4911 & 0.4421 & 0.4891 & 0.4403 & 0.004024 \\
0.2134 & 0.2442 & 0.2128 & 0.2435 & 0.002804 \\
0.7214 & 0.1835 & 0.7168 & 0.1823 & 0.006332
\end{tabular}

\begin{tabular}{lllll}
0.5299 & 0.3067 & 0.5273 & 0.3052 & 0.004814 \\
0.1573 & 0.3073 & 0.1569 & 0.3066 & 0.002344 \\
0.1135 & 0.7663 & 0.1134 & 0.7654 & 0.001225 \\
0.2785 & 0.1774 & 0.2776 & 0.1768 & 0.003318 \\
0.1279 & 0.5364 & 0.1277 & 0.5354 & 0.001798 \\
0.4940 & 0.1587 & 0.4915 & 0.1579 & 0.004969 \\
0.5810 & 0.1525 & 0.5776 & 0.1516 & 0.005899 \\
\hline${ }^{{ }^{a} x_{3}{ }^{(\mathrm{s})}: \text { initial mole fraction of component } 3 \text { in the ternary solvent mixture, } x_{4}{ }^{(\mathrm{s})}}:$ \\
initial mole fraction of component 4 in the ternary solvent mixture; $x_{1}:$ mole \\
fraction solubility of the solute; $x_{3}:$ mole fraction of component 3 in the \\
quaternary solution; $x_{4}:$ mole fraction of component 4 in the quaternary \\
solution.
\end{tabular}

\section{Auxiliary Information}

\begin{abstract}
Method/Apparatus/Procedure:
Constant-temperature bath, calorimetric thermometer, and an ultraviolet/ visible spectrophotometer.

Ternary solvent mixtures were prepared by mass. Excess solute and solvent were placed in amber glass bottles and allowed to equilibrate for several days at constant temperature. Attainment of equilibrium was verified by several repetitive measurements and by approaching equilibrium from supersaturation. Aliquots of saturated solutions were transferred through a coarse filter into volumetric flasks, weighed and diluted with methanol. Molar concentrations were determined by spectrophotometric measurements at $356 \mathrm{~nm}$.
\end{abstract}

\section{Source and Purity of Chemicals:}

(1) $99+\%$, Aldrich Chemical Company, Milwaukee, WI, USA, was used as received.

(2) $99.9+\%$, HPLC grade, Aldrich Chemical Company.

(3) $99.8 \%$, anhydrous, Aldrich Chemical Company.

(4) $99+\%$, anhydrous, Aldrich Chemical Company.

Components 2-4 were stored over molecular sieves and distilled shortly before use.

\section{Estimated Error:}

Temperature: $\pm 0.1 \mathrm{~K}$.

$x_{3}{ }^{(\mathrm{s})}: \pm 0.0001$.

$x_{4}{ }^{(\mathrm{s})}: \pm 0.0001$.

$x_{1}: \pm 1.5 \%$ (relative error).

\begin{tabular}{ll}
\hline \hline Components: & Original Measurements: \\
(1) Anthracene; $\mathrm{C}_{14} \mathrm{H}_{10} ;[120-12-7]$ & ${ }^{13}$ K. J. Pribyla, M. A. Spurgin, I. \\
(2) Heptane; $\mathrm{C}_{7} \mathrm{H}_{16} ;[142-82-5]$ & Chuca, and W. E. Acree, Jr., J. \\
(3) 1,4-Dioxane; $\mathrm{C}_{4} \mathrm{H}_{8} \mathrm{O}_{2} ;[123-91-1]$ & Chem. Eng. Data 45, 965 (2000). \\
(4) 1-Butanol; $\mathrm{C}_{4} \mathrm{H}_{10} \mathrm{O} ;[71-36-3]$ & \\
\hline Variables: & Prepared by: \\
$T / \mathrm{K}=298.15 ;$ Solvent Composition & W. E. Acree, Jr. \\
\hline
\end{tabular}


Experimental Values

Solubility of anthracene in heptane +1 ,4-dioxane +1 -butanol mixtures ${ }^{\mathrm{a}}$

\begin{tabular}{lcccc}
\hline \hline$x_{3}{ }^{(\mathrm{s})}$ & $x_{4}{ }^{(\mathrm{s})}$ & $x_{3}$ & $x_{4}$ & $x_{1}$ \\
\hline 0.0000 & 0.0000 & 0.0000 & 0.0000 & 0.001571 \\
1.0000 & 0.0000 & 0.9917 & 0.0000 & 0.008329 \\
0.0000 & 1.0000 & 0.0000 & 0.9992 & 0.000801 \\
0.3978 & 0.3721 & 0.3962 & 0.3706 & 0.004077 \\
0.1791 & 0.7173 & 0.1788 & 0.7159 & 0.001927 \\
0.3311 & 0.3028 & 0.3299 & 0.3017 & 0.003643 \\
0.2954 & 0.5348 & 0.2945 & 0.5332 & 0.003049 \\
0.7559 & 0.1144 & 0.7506 & 0.1136 & 0.007020 \\
0.7332 & 0.2023 & 0.7286 & 0.2010 & 0.006308 \\
0.2174 & 0.7125 & 0.2169 & 0.7110 & 0.002165 \\
0.1372 & 0.5726 & 0.1369 & 0.5714 & 0.002046 \\
0.4316 & 0.4963 & 0.4300 & 0.4944 & 0.003815 \\
0.5311 & 0.4000 & 0.5287 & 0.3982 & 0.004590 \\
0.2677 & 0.2487 & 0.2668 & 0.2479 & 0.003252 \\
0.7456 & 0.1586 & 0.7407 & 0.1575 & 0.006637 \\
0.5647 & 0.2682 & 0.5617 & 0.2668 & 0.005332 \\
0.1538 & 0.2737 & 0.1534 & 0.2730 & 0.002415 \\
0.1269 & 0.7392 & 0.1267 & 0.7380 & 0.001638 \\
0.2913 & 0.1419 & 0.2903 & 0.1414 & 0.003510 \\
0.1500 & 0.4790 & 0.1497 & 0.4779 & 0.002254 \\
0.5006 & 0.1305 & 0.4980 & 0.1298 & 0.005255 \\
0.5964 & 0.1223 & 0.5927 & 0.1215 & 0.006243 \\
\hline$x_{3}(\mathrm{~s}): 1 \mathrm{~s}$ & &
\end{tabular}

${ }^{\mathrm{a}} x_{3}{ }^{(\mathrm{s})}$ : initial mole fraction of component 3 in the ternary solvent mixture, $x_{4}{ }^{(\mathrm{s})}$ : initial mole fraction of component 4 in the ternary solvent mixture; $x_{1}$ : mole fraction solubility of the solute; $x_{3}$ : mole fraction of component 3 in the quaternary solution; $x_{4}$ : mole fraction of component 4 in the quaternary solution.

\section{Auxiliary Information}

\section{Method/Apparatus/Procedure:}

Constant-temperature bath, calorimetric thermometer, and an ultraviolet/ visible spectrophotometer.

Ternary solvent mixtures were prepared by mass. Excess solute and solvent were placed in amber glass bottles and allowed to equilibrate for several days at constant temperature. Attainment of equilibrium was verified by several repetitive measurements and by approaching equilibrium from supersaturation. Aliquots of saturated solutions were transferred through a coarse filter into volumetric flasks, weighed and diluted with methanol. Molar concentrations were determined by spectrophotometric measurements at $356 \mathrm{~nm}$.

\section{Source and Purity of Chemicals:}

(1) $99+\%$, Aldrich Chemical Company, Milwaukee, WI, USA, was used as received.

(2) $99.9+\%$, HPLC grade, Aldrich Chemical Company.

(3) $99.8 \%$, anhydrous, Aldrich Chemical Company.

(4) $99.8+\%$, HPLC grade, Aldrich Chemical Company.

Components $2-4$ were stored over molecular sieves and distilled shortly before use.

Estimated Error:

Temperature: $\pm 0.1 \mathrm{~K}$.

$x_{3}{ }^{(\mathrm{s})}: \pm 0.0001$

$x_{4}{ }^{(\mathrm{s})}: \pm 0.0001$

$x_{1}: \pm 1.5 \%$ (relative error).

\begin{tabular}{|c|c|}
\hline $\begin{array}{l}\text { Components: } \\
\text { (1) Anthracene; } \mathrm{C}_{14} \mathrm{H}_{10} ;[120-12-7] \\
\text { (2) Heptane; } \mathrm{C}_{7} \mathrm{H}_{16} ;[142-82-5] \\
\text { (3) 1,4-Dioxane; } \mathrm{C}_{4} \mathrm{H}_{8} \mathrm{O}_{2} ;[123-91-1] \\
\text { (4) 2-Butanol; } \mathrm{C}_{4} \mathrm{H}_{10} \mathrm{O} ;[\text { [78-92-2] }\end{array}$ & $\begin{array}{l}\text { Original Measurements: } \\
{ }^{13} \text { K. J. Pribyla, M. A. Spurgin, I. } \\
\text { Chuca, and W. E. Acree, Jr., J. } \\
\text { Chem. Eng. Data 45, } 965 \text { (2000). }\end{array}$ \\
\hline $\begin{array}{l}\text { Variables: } \\
T / \mathrm{K}=298.15 ; \text { Solvent Composition }\end{array}$ & $\begin{array}{l}\text { Prepared by: } \\
\text { W. E. Acree, Jr. }\end{array}$ \\
\hline
\end{tabular}

Experimental Values

Solubility of anthracene in heptane +1 ,4-dioxane +2 -butanol mixtures ${ }^{\mathrm{a}}$

\begin{tabular}{lcccc}
\hline \hline$x_{3}{ }^{(\mathrm{s})}$ & $x_{4}{ }^{(\mathrm{s})}$ & $x_{3}$ & $x_{4}$ & $x_{1}$ \\
\hline 0.0000 & 0.0000 & 0.0000 & 0.0000 & 0.001571 \\
1.0000 & 0.0000 & 0.9917 & 0.0000 & 0.008329 \\
0.0000 & 1.0000 & 0.0000 & 0.9994 & 0.000585 \\
0.3958 & 0.3749 & 0.3942 & 0.3734 & 0.003924 \\
0.1755 & 0.7208 & 0.1752 & 0.7195 & 0.001766 \\
0.3299 & 0.3063 & 0.3287 & 0.3052 & 0.003605 \\
0.2952 & 0.5319 & 0.2943 & 0.5303 & 0.002922 \\
0.7658 & 0.1021 & 0.7603 & 0.1014 & 0.007224 \\
0.7317 & 0.1986 & 0.7270 & 0.1973 & 0.006391 \\
0.2285 & 0.7000 & 0.2280 & 0.6985 & 0.002135 \\
0.1424 & 0.5685 & 0.1421 & 0.5674 & 0.002015 \\
0.4323 & 0.4953 & 0.4307 & 0.4935 & 0.003699 \\
0.5372 & 0.3914 & 0.5347 & 0.2896 & 0.004569 \\
0.2280 & 0.2060 & 0.2273 & 0.2054 & 0.002913 \\
0.7494 & 0.1508 & 0.7443 & 0.1498 & 0.006777 \\
0.5642 & 0.2633 & 0.5613 & 0.2619 & 0.005162 \\
0.1549 & 0.2741 & 0.1545 & 0.2734 & 0.002456 \\
0.1214 & 0.7442 & 0.1212 & 0.7431 & 0.001536 \\
0.2935 & 0.1365 & 0.2924 & 0.1360 & 0.003614 \\
0.1460 & 0.4828 & 0.1457 & 0.4817 & 0.002226 \\
0.5046 & 0.1277 & 0.5019 & 0.1270 & 0.005263 \\
0.5973 & 0.1211 & 0.5936 & 0.1204 & 0.006181 \\
\hline${ }_{1}{ }^{(\mathrm{s})}:$ & &
\end{tabular}

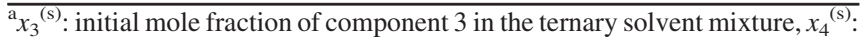
initial mole fraction of component 4 in the ternary solvent mixture; $x_{1}$ : mole fraction solubility of the solute; $x_{3}$ : mole fraction of component 3 in the quaternary solution; $x_{4}$ : mole fraction of component 4 in the quaternary solution.

\section{Auxiliary Information}

\section{Method/Apparatus/Procedure:}

Constant-temperature bath, calorimetric thermometer, and an ultraviolet/ visible spectrophotometer.

Ternary solvent mixtures were prepared by mass. Excess solute and solvent were placed in amber glass bottles and allowed to equilibrate for several days at constant temperature. Attainment of equilibrium was verified by several repetitive measurements and by approaching equilibrium from supersaturation. Aliquots of saturated solutions were transferred through a coarse filter into volumetric flasks, weighed and diluted with methanol. Molar concentrations were determined by spectrophotometric measurements at $356 \mathrm{~nm}$.

Source and Purity of Chemicals:

(1) $99+\%$, Aldrich Chemical Company, Milwaukee, WI, USA, was used as received.

(2) $99.9+\%$, HPLC grade, Aldrich Chemical Company.

(3) $99.8 \%$, anhydrous, Aldrich Chemical Company.

(4) $99+\%$, anhydrous, Aldrich Chemical Company.

Components $2-4$ were stored over molecular sieves and distilled shortly before use. 
Estimated Error:

Temperature: $\pm 0.1 \mathrm{~K}$

$x_{3}{ }^{(\mathrm{s})}: \pm 0.0001$.

$x_{4}{ }^{(\mathrm{s})}: \pm 0.0001$.

$x_{1}: \pm 1.5 \%$ (relative error)

\begin{tabular}{|c|c|}
\hline $\begin{array}{l}\text { Components: } \\
\text { (1) Anthracene; } \mathrm{C}_{14} \mathrm{H}_{10} ;[120-12-7] \\
\text { (2) Heptane; } \mathrm{C}_{7} \mathrm{H}_{16} ;[142-82-5] \\
\text { (3) 1,4-Dioxane; } \mathrm{C}_{4} \mathrm{H}_{8} \mathrm{O}_{2} ;[123-91-1] \\
\text { (4) 2-Methyl-1-propanol; } \mathrm{C}_{4} \mathrm{H}_{10} \mathrm{O} \text {; } \\
\text { [78-83-1] }\end{array}$ & $\begin{array}{l}\text { Original Measurements: } \\
{ }^{13} \text { K. J. Pribyla, M. A. Spurgin, I. } \\
\text { Chuca, and W. E. Acree, Jr., J. } \\
\text { Chem. Eng. Data 45, } 965 \text { (2000). }\end{array}$ \\
\hline $\begin{array}{l}\text { Variables: } \\
T / \mathrm{K}=298.15 ; \text { Solvent Composition }\end{array}$ & $\begin{array}{l}\text { Prepared by: } \\
\text { W. E. Acree, Jr. }\end{array}$ \\
\hline
\end{tabular}

\section{Experimental Values}

Solubility of anthracene in heptane +1 ,4-dioxane +2 -methyl-1-propanol mixtures $^{\mathrm{a}}$

\begin{tabular}{|c|c|c|c|c|}
\hline$x_{3}{ }^{(\mathrm{s})}$ & $x_{4}^{(\mathrm{s})}$ & $x_{3}$ & $x_{4}$ & $x_{1}$ \\
\hline 0.0000 & 0.0000 & 0.0000 & 0.0000 & 0.001571 \\
\hline 1.0000 & 0.0000 & 0.9917 & 0.0000 & 0.008329 \\
\hline 0.0000 & 1.0000 & 0.0000 & 0.9995 & 0.000470 \\
\hline 0.4012 & 0.3672 & 0.3997 & 0.3658 & 0.003745 \\
\hline 0.1782 & 0.7163 & 0.1779 & 0.7152 & 0.001567 \\
\hline 0.3714 & 0.2850 & 0.3700 & 0.2839 & 0.003805 \\
\hline 0.3100 & 0.5193 & 0.3091 & 0.5179 & 0.002760 \\
\hline 0.7631 & 0.1072 & 0.7578 & 0.1065 & 0.006943 \\
\hline 0.7344 & 0.1962 & 0.7298 & 0.1950 & 0.006208 \\
\hline 0.2270 & 0.7036 & 0.2266 & 0.7023 & 0.001791 \\
\hline 0.1515 & 0.5619 & 0.1512 & 0.5609 & 0.001860 \\
\hline 0.4411 & 0.4901 & 0.4396 & 0.4884 & 0.003459 \\
\hline 0.5318 & 0.3966 & 0.5295 & 0.3949 & 0.004341 \\
\hline 0.2283 & 0.2030 & 0.2276 & 0.2024 & 0.002919 \\
\hline 0.7456 & 0.1544 & 0.7405 & 0.1533 & 0.006841 \\
\hline 0.5612 & 0.2702 & 0.5584 & 0.2688 & 0.005041 \\
\hline 0.1623 & 0.2702 & 0.1619 & 0.2695 & 0.002415 \\
\hline 0.1308 & 0.7330 & 0.1306 & 0.7320 & 0.001310 \\
\hline 0.2986 & 0.1420 & 0.2976 & 0.1415 & 0.003492 \\
\hline 0.1437 & 0.4852 & 0.1434 & 0.4842 & 0.001971 \\
\hline 0.5166 & 0.1284 & 0.5138 & 0.1277 & 0.005369 \\
\hline 0.6000 & 0.1242 & 0.5964 & 0.1235 & 0.005988 \\
\hline \multicolumn{5}{|c|}{$\begin{array}{l}{ }^{a} x_{3}{ }_{3}^{(\mathrm{s})} \text { : initial mole fraction of component } 3 \text { in the ternary solvent mixture, } x_{4} \\
\text { initial mole fraction of component } 4 \text { in the ternary solvent mixture; } x_{1}: \text { mo } \\
\text { fraction solubility of the solute; } x_{3}: \text { mole fraction of component } 3 \text { in the } \\
\text { quaternary solution; } x_{4} \text { : mole fraction of component } 4 \text { in the quaternary } \\
\text { solution. }\end{array}$} \\
\hline
\end{tabular}

\section{Auxiliary Information}

\section{Method/Apparatus/Procedure:}

Constant-temperature bath, calorimetric thermometer, and an ultraviolet/ visible spectrophotometer.

Ternary solvent mixtures were prepared by mass. Excess solute and solvent were placed in amber glass bottles and allowed to equilibrate for several days at constant temperature. Attainment of equilibrium was verified by several repetitive measurements and by approaching equilibrium from supersaturation. Aliquots of saturated solutions were transferred through a coarse filter into volumetric flasks, weighed and diluted with methanol. Molar concentrations were determined by spectrophotometric measurements at $356 \mathrm{~nm}$.
Source and Purity of Chemicals:

(1) $99+\%$, Aldrich Chemical Company, Milwaukee, WI, USA, was used as received.

(2) $99.9+\%$, HPLC grade, Aldrich Chemical Company.

(3) $99.9 \%$, Arco Chemical Company, USA.

(4) $99+\%$, anhydrous, Aldrich Chemical Company.

Components 2-4 were stored over molecular sieves and distilled shortly before use.

\section{Estimated Error:}

Temperature: $\pm 0.1 \mathrm{~K}$.

$x_{3}{ }^{(s)}: \pm 0.0001$.

$x_{4}{ }^{(\mathrm{s})}: \pm 0.0001$.

$x_{1}: \pm 1.5 \%$ (relative error).

\section{Components:}

(1) Anthracene; $\mathrm{C}_{14} \mathrm{H}_{10} ;[120-12-7] \quad{ }^{14}$ K. J. Pribyla, M. A. Spurgin, I.

(2) Cyclohexane; $\mathrm{C}_{6} \mathrm{H}_{12} ;[110-82-7] \quad$ Chuca, and W. E. Acree, Jr., J.

(3) 1,4-Dioxane; $\mathrm{C}_{4} \mathrm{H}_{8} \mathrm{O}_{2}$; [123-91-1] Chem. Eng. Data 45, 971 (2000).

(4) 1-Propanol; $\mathrm{C}_{3} \mathrm{H}_{8} \mathrm{O}$; [71-23-8]

\begin{tabular}{ll}
\hline Variables: & Prepared by: \\
$T / \mathrm{K}=298.15 ;$ Solvent Composition & W. E. Acree, Jr. \\
\hline
\end{tabular}

Experimental Values

Solubility of anthracene in cyclohexane $+1,4$-dioxane +1 -propanol mixtures ${ }^{\mathrm{a}}$

\begin{tabular}{lcccc}
\hline \hline$x_{3}{ }^{(\mathrm{s})}$ & $x_{4}{ }^{(\mathrm{s})}$ & $x_{3}$ & $x_{4}$ & $x_{1}$ \\
\hline 0.0000 & 0.0000 & 0.0000 & 0.0000 & 0.001553 \\
1.0000 & 0.0000 & 0.9917 & 0.0000 & 0.008329 \\
0.0000 & 1.0000 & 0.0000 & 0.9994 & 0.000591 \\
0.3419 & 0.3906 & 0.3406 & 0.3891 & 0.003921 \\
0.1437 & 0.7402 & 0.1435 & 0.7390 & 0.001604 \\
0.2742 & 0.3107 & 0.2732 & 0.3096 & 0.003625 \\
0.2542 & 0.5457 & 0.2535 & 0.5442 & 0.002793 \\
0.7091 & 0.1240 & 0.7039 & 0.1231 & 0.007311 \\
0.6853 & 0.2293 & 0.6809 & 0.2278 & 0.006388 \\
0.1887 & 0.7314 & 0.1884 & 0.7300 & 0.001845 \\
0.1125 & 0.5739 & 0.1123 & 0.5728 & 0.001866 \\
0.3795 & 0.5369 & 0.3782 & 0.5350 & 0.003456 \\
0.4784 & 0.4381 & 0.4763 & 0.4362 & 0.004384 \\
0.1771 & 0.2058 & 0.1766 & 0.2052 & 0.003079 \\
0.6976 & 0.1803 & 0.6929 & 0.1791 & 0.006711 \\
0.5025 & 0.2936 & 0.4998 & 0.2920 & 0.005290 \\
0.1199 & 0.2684 & 0.1196 & 0.2677 & 0.002502 \\
0.1043 & 0.7444 & 0.1041 & 0.7433 & 0.001443 \\
0.2339 & 0.1445 & 0.2330 & 0.1440 & 0.003702 \\
0.1165 & 0.4773 & 0.1163 & 0.4763 & 0.002106 \\
0.4377 & 0.1362 & 0.4353 & 0.1354 & 0.005574 \\
0.5291 & 0.1354 & 0.5257 & 0.1345 & 0.006337 \\
\hline$x_{3}{ }^{(\mathrm{s})}:$ & &
\end{tabular}

${ }^{\mathrm{a}} x_{3}{ }^{(\mathrm{s})}$ : initial mole fraction of component 3 in the ternary solvent mixture, $x_{4}{ }^{(\mathrm{s})}$ : initial mole fraction of component 4 in the ternary solvent mixture; $x_{1}$ : mole fraction solubility of the solute; $x_{3}$ : mole fraction of component 3 in the quaternary solution; $x_{4}$ : mole fraction of component 4 in the quaternary solution. 


\section{Auxiliary Information}

Method/Apparatus/Procedure:

Constant-temperature bath, calorimetric thermometer, and an ultraviolet/ visible spectrophotometer.

Ternary solvent mixtures were prepared by mass. Excess solute and solvent were placed in amber glass bottles and allowed to equilibrate for several days at constant temperature. Attainment of equilibrium was verified by several repetitive measurements and by approaching equilibrium from supersaturation. Aliquots of saturated solutions were transferred through a coarse filter into volumetric flasks, weighed and diluted with methanol. Molar concentrations were determined by spectrophotometric measurements at $356 \mathrm{~nm}$.

\section{Source and Purity of Chemicals:}

(1) $99.9+\%$, Aldrich Chemical Company, Milwaukee, WI, USA, was used as received.

(2) $99.9+\%$, HPLC grade, Aldrich Chemical Company.

(3) $99.8 \%$, anhydrous, Aldrich Chemical Company.

(4) $99+\%$, anhydrous, Aldrich Chemical Company.

Components 2-4 were stored over molecular sieves and distilled shortly before use.

\section{Estimated Error:}

Temperature: $\pm 0.1 \mathrm{~K}$.

$x_{3}{ }^{(\mathrm{s})}: \pm 0.0001$.

$x_{4}{ }^{(\mathrm{s})}: \pm 0.0001$.

$x_{1}: \pm 1.5 \%$ (relative error).

\section{Components:}

(1) Anthracene; $\mathrm{C}_{14} \mathrm{H}_{10} ;[120-12-7]$

(2) Cyclohexane; $\mathrm{C}_{6} \mathrm{H}_{12} ;$ [110-82-7]

Original Measurements:

${ }^{14}$ K. J. Pribyla, M. A. Spurgin, I.

(3) 1,4-Dioxane; $\mathrm{C}_{4} \mathrm{H}_{8} \mathrm{O}_{2}$; [123-91-1]

Chuca, and W. E. Acree, Jr., J.

Chem. Eng. Data 45, 971 (2000).

(4) 2-Propanol; $\mathrm{C}_{3} \mathrm{H}_{8} \mathrm{O}$; [67-63-0]

\begin{tabular}{ll}
\hline Variables: & Prepared by: \\
$T / \mathrm{K}=298.15 ;$ Solvent Composition & W. E. Acree, Jr. \\
\hline
\end{tabular}

Experimental Values

Solubility of anthracene in cyclohexane +1 ,4-dioxane +2 -propanol mixtures ${ }^{\mathrm{a}}$

\begin{tabular}{lcccc}
\hline \hline$x_{3}{ }^{(\mathrm{s})}$ & $x_{4}{ }^{(\mathrm{s})}$ & $x_{3}$ & $x_{4}$ & $x_{1}$ \\
\hline 0.0000 & 0.0000 & 0.0000 & 0.0000 & 0.001553 \\
1.0000 & 0.0000 & 0.9917 & 0.0000 & 0.008329 \\
0.0000 & 1.0000 & 0.0000 & 0.9996 & 0.000411 \\
0.3454 & 0.3819 & 0.3441 & 0.3805 & 0.003745 \\
0.1522 & 0.7289 & 0.1520 & 0.7278 & 0.001449 \\
0.2732 & 0.3062 & 0.2722 & 0.3051 & 0.003510 \\
0.2472 & 0.5579 & 0.2466 & 0.5565 & 0.002514 \\
0.7089 & 0.1240 & 0.7039 & 0.1231 & 0.007040 \\
0.6887 & 0.2255 & 0.6845 & 0.2241 & 0.006059 \\
0.1907 & 0.7289 & 0.1904 & 0.7277 & 0.001636 \\
0.1124 & 0.5677 & 0.1122 & 0.5667 & 0.001771 \\
0.3806 & 0.5317 & 0.3784 & 0.5300 & 0.003211
\end{tabular}

\begin{tabular}{lllll}
0.4838 & 0.4300 & 0.4819 & 0.4283 & 0.003943 \\
0.1840 & 0.1975 & 0.1834 & 0.1969 & 0.003121 \\
0.7017 & 0.1760 & 0.6971 & 0.1748 & 0.006600 \\
0.5041 & 0.2918 & 0.5015 & 0.2903 & 0.005073 \\
0.1245 & 0.2594 & 0.1242 & 0.2587 & 0.002507 \\
0.1045 & 0.7401 & 0.1044 & 0.7392 & 0.001248 \\
0.2388 & 0.1420 & 0.2379 & 0.1415 & 0.003656 \\
0.1177 & 0.4675 & 0.1175 & 0.4666 & 0.002004 \\
0.4414 & 0.1324 & 0.4390 & 0.1317 & 0.005403 \\
0.5309 & 0.1327 & 0.5276 & 0.1319 & 0.006263 \\
\hline${ }^{a} x_{3}{ }^{(s)}:$ initial mole fraction of component 3 in the ternary solvent mixture, $x_{4}{ }^{\left({ }^{(s)}\right.}$ : \\
initial mole fraction of component 4 in the ternary solvent mixture; $x_{1}:$ mole \\
fraction solubility of the solute; $x_{3}:$ mole fraction of component 3 in the \\
quaternary solution; $x_{4}:$ mole fraction of component 4 in the quaternary \\
solution.
\end{tabular}

\section{Auxiliary Information}

Method/Apparatus/Procedure:

Constant-temperature bath, calorimetric thermometer, and an ultraviolet/ visible spectrophotometer.

Ternary solvent mixtures were prepared by mass. Excess solute and solvent were placed in amber glass bottles and allowed to equilibrate for several days at constant temperature. Attainment of equilibrium was verified by several repetitive measurements and by approaching equilibrium from supersaturation. Aliquots of saturated solutions were transferred through a coarse filter into volumetric flasks, weighed and diluted with methanol. Molar concentrations were determined by spectrophotometric measurements at $356 \mathrm{~nm}$.

Source and Purity of Chemicals:

(1) $99+\%$, Aldrich Chemical Company, Milwaukee, WI, USA, was used as received.

(2) $99.9+\%$, HPLC grade, Aldrich Chemical Company.

(3) $99.8 \%$, anhydrous, Aldrich Chemical Company.

(4) $99+\%$, anhydrous, Aldrich Chemical Company.

Components 2-4 were stored over molecular sieves and distilled shortly before use.

\section{Estimated Error:}

Temperature: $\pm 0.1 \mathrm{~K}$.

$x_{3}{ }^{(\mathrm{s})}: \pm 0.0001$.

$x_{4}{ }^{(\mathrm{s})}: \pm 0.0001$.

$x_{1}: \pm 1.5 \%$ (relative error).

\begin{tabular}{ll}
\hline \hline Components: & Original Measurements: \\
(1) Anthracene; $\mathrm{C}_{14} \mathrm{H}_{10} ;[120-12-7]$ & ${ }^{14} \mathrm{~K}$. J. Pribyla, M. A. Spurgin, I. \\
(2) Cyclohexane; $\mathrm{C}_{6} \mathrm{H}_{12} ;[110-82-7]$ & Chuca, and W. E. Acree, Jr., J. \\
(3) 1,4-Dioxane; $\mathrm{C}_{4} \mathrm{H}_{8} \mathrm{O}_{2} ;[123-91-1]$ & Chem. Eng. Data 45, 971 (2000). \\
(4) 1-Butanol; $\mathrm{C}_{4} \mathrm{H}_{10} \mathrm{O} ;[71-36-3]$ & \\
\hline Variables: & Prepared by: \\
$T / \mathrm{K}=298.15 ;$ Solvent Composition & W. E. Acree, Jr. \\
\hline
\end{tabular}




\section{Experimental Values}

Solubility of anthracene in cyclohexane +1 ,4-dioxane +1 -butanol mixtures ${ }^{\mathrm{a}}$

\begin{tabular}{lcccc}
\hline \hline$x_{3}{ }^{(\mathrm{s})}$ & $x_{4}{ }^{(\mathrm{s})}$ & $x_{3}$ & $x_{4}$ & $x_{1}$ \\
\hline 0.0000 & 0.0000 & 0.0000 & 0.0000 & 0.001553 \\
1.0000 & 0.0000 & 0.9917 & 0.0000 & 0.008329 \\
0.0000 & 1.0000 & 0.0000 & 0.9992 & 0.000801 \\
0.3714 & 0.3413 & 0.3698 & 0.3399 & 0.004202 \\
0.1720 & 0.6948 & 0.1717 & 0.6934 & 0.001972 \\
0.2908 & 0.2681 & 0.2896 & 0.2670 & 0.004046 \\
0.2791 & 0.5032 & 0.2782 & 0.5016 & 0.003094 \\
0.7231 & 0.1074 & 0.7176 & 0.1066 & 0.007562 \\
0.7159 & 0.1952 & 0.7109 & 0.1938 & 0.006997 \\
0.2179 & 0.6892 & 0.2174 & 0.6877 & 0.002197 \\
0.1276 & 0.5197 & 0.1273 & 0.5186 & 0.002149 \\
0.4196 & 0.4873 & 0.4180 & 0.4854 & 0.003898 \\
0.5203 & 0.3880 & 0.5178 & 0.3861 & 0.004816 \\
0.1852 & 0.1713 & 0.1846 & 0.1707 & 0.003235 \\
0.7253 & 0.1460 & 0.7200 & 0.1449 & 0.007327 \\
0.5313 & 0.2525 & 0.5283 & 0.2510 & 0.005721 \\
0.1316 & 0.2234 & 0.1313 & 0.2228 & 0.002654 \\
0.1199 & 0.7040 & 0.1197 & 0.7028 & 0.001725 \\
0.2390 & 0.1234 & 0.2381 & 0.1229 & 0.003779 \\
0.1300 & 0.4238 & 0.1297 & 0.4228 & 0.002362 \\
0.4455 & 0.1127 & 0.4430 & 0.1121 & 0.005672 \\
0.5417 & 0.1124 & 0.5382 & 0.1117 & 0.006553 \\
\hline
\end{tabular}

${ }^{\mathrm{a}} x_{3}{ }^{(\mathrm{s})}$ : initial mole fraction of component 3 in the ternary solvent mixture, $x_{4}{ }^{(\mathrm{s})}$ : initial mole fraction of component 4 in the ternary solvent mixture; $x_{1}$ : mole fraction solubility of the solute; $x_{3}$ : mole fraction of component 3 in the quaternary solution; $x_{4}$ : mole fraction of component 4 in the quaternary solution.

\section{Auxiliary Information}

\section{Method/Apparatus/Procedure:}

Constant-temperature bath, calorimetric thermometer, and an ultraviolet/ visible spectrophotometer.

Ternary solvent mixtures were prepared by mass. Excess solute and solvent were placed in amber glass bottles and allowed to equilibrate for several days at constant temperature. Attainment of equilibrium was verified by several repetitive measurements and by approaching equilibrium from supersaturation. Aliquots of saturated solutions were transferred through a coarse filter into volumetric flasks, weighed and diluted with methanol. Molar concentrations were determined by spectrophotometric measurements at $356 \mathrm{~nm}$.

\section{Source and Purity of Chemicals:}

(1) $99+\%$, Aldrich Chemical Company, Milwaukee, WI, USA, was used as received.

(2) $99.9+\%$, HPLC grade, Aldrich Chemical Company.

(3) $99.8 \%$, anhydrous, Aldrich Chemical Company.

(4) $99.8+\%$, HPLC grade, Aldrich Chemical Company.

Components $2-4$ were stored over molecular sieves and distilled shortly before use.

\section{Estimated Error:}

Temperature: $\pm 0.1 \mathrm{~K}$.

$x_{3}{ }^{(\mathrm{s})}: \pm 0.0001$

$x_{4}^{(\mathrm{s})}: \pm 0.0001$

$x_{1}: \pm 1.5 \%$ (relative error)

\begin{tabular}{|c|c|}
\hline $\begin{array}{l}\text { Components: } \\
\text { (1) Anthracene; } \mathrm{C}_{14} \mathrm{H}_{10} ;[120-12-7] \\
\text { (2) Cyclohexane; } \mathrm{C}_{6} \mathrm{H}_{12} ;[110-82-7] \\
\text { (3) 1,4-Dioxane; } \mathrm{C}_{4} \mathrm{H}_{8} \mathrm{O}_{2} ;[123-91-1] \\
\text { (4) 2-Butanol; } \mathrm{C}_{4} \mathrm{H}_{10} \mathrm{O} ;[78-92-2]\end{array}$ & $\begin{array}{l}\text { Original Measurements: } \\
{ }^{14} \text { K. J. Pribyla, M. A. Spurgin, I. } \\
\text { Chuca, and W. E. Acree, Jr., J. } \\
\text { Chem. Eng. Data 45, } 971 \text { (2000). }\end{array}$ \\
\hline $\begin{array}{l}\text { Variables: } \\
T / \mathrm{K}=298.15 \text {; Solvent Composition }\end{array}$ & $\begin{array}{l}\text { Prepared by: } \\
\text { W. E. Acree, Jr. }\end{array}$ \\
\hline
\end{tabular}

Experimental Values

Solubility of anthracene in cyclohexane +1 ,4-dioxane +2 -butanol mixtures ${ }^{\mathrm{a}}$

\begin{tabular}{lcccc}
\hline \hline$x_{3}{ }^{(\mathrm{s})}$ & $x_{4}{ }^{(\mathrm{s})}$ & $x_{3}$ & $x_{4}$ & $x_{1}$ \\
\hline 0.0000 & 0.0000 & 0.0000 & 0.0000 & 0.001553 \\
1.0000 & 0.0000 & 0.9917 & 0.0000 & 0.008329 \\
0.0000 & 1.0000 & 0.0000 & 0.9994 & 0.000585 \\
0.3227 & 0.4227 & 0.3215 & 0.4212 & 0.003575 \\
0.1682 & 0.7013 & 0.1679 & 0.7000 & 0.001818 \\
0.2937 & 0.2668 & 0.2926 & 0.2657 & 0.003890 \\
0.2778 & 0.5023 & 0.2769 & 0.5007 & 0.003098 \\
0.7240 & 0.1099 & 0.7187 & 0.1091 & 0.007256 \\
0.7141 & 0.1970 & 0.7093 & 0.1957 & 0.006709 \\
0.2203 & 0.6864 & 0.2198 & 0.6849 & 0.002124 \\
0.1254 & 0.5180 & 0.1251 & 0.5169 & 0.002080 \\
0.4220 & 0.4836 & 0.4204 & 0.4818 & 0.003779 \\
0.5181 & 0.3865 & 0.5157 & 0.3847 & 0.004627 \\
0.1827 & 0.1763 & 0.1821 & 0.1757 & 0.003137 \\
0.7201 & 0.1510 & 0.7150 & 0.1499 & 0.007111 \\
0.5324 & 0.2521 & 0.5294 & 0.2507 & 0.005721 \\
0.1334 & 0.2259 & 0.1330 & 0.2253 & 0.002723 \\
0.1197 & 0.7054 & 0.1195 & 0.7043 & 0.001600 \\
0.2401 & 0.1226 & 0.2392 & 0.1221 & 0.003790 \\
0.1281 & 0.4236 & 0.1278 & 0.4226 & 0.002310 \\
0.4492 & 0.1133 & 0.4467 & 0.1127 & 0.005575 \\
0.5420 & 0.1115 & 0.5386 & 0.1108 & 0.006332 \\
\hline
\end{tabular}

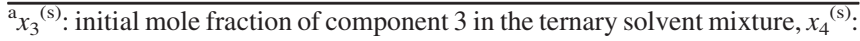
initial mole fraction of component 4 in the ternary solvent mixture; $x_{1}$ : mole fraction solubility of the solute; $x_{3}$ : mole fraction of component 3 in the quaternary solution; $x_{4}$ : mole fraction of component 4 in the quaternary solution.

\section{Auxiliary Information}

\section{Method/Apparatus/Procedure:}

Constant-temperature bath, calorimetric thermometer, and an ultraviolet/ visible spectrophotometer.

Ternary solvent mixtures were prepared by mass. Excess solute and solvent were placed in amber glass bottles and allowed to equilibrate for several days at constant temperature. Attainment of equilibrium was verified by several repetitive measurements and by approaching equilibrium from supersaturation. Aliquots of saturated solutions were transferred through a coarse filter into volumetric flasks, weighed and diluted with methanol. Molar concentrations were determined by spectrophotometric measurements at $356 \mathrm{~nm}$.

Source and Purity of Chemicals:

(1) $99+\%$, Aldrich Chemical Company, Milwaukee, WI, USA, was used as received.

(2) $99.9+\%$, HPLC grade, Aldrich Chemical Company.

(3) $99.8 \%$, anhydrous, Aldrich Chemical Company.

(4) $99+\%$, anhydrous, Aldrich Chemical Company.

Components 2-4 were stored over molecular sieves and distilled shortly before use. 
Estimated Error:

Temperature: $\pm 0.1 \mathrm{~K}$.

$x_{3}{ }^{(\mathrm{s})}: \pm 0.0001$.

$x_{4}{ }^{(\mathrm{s})}: \pm 0.0001$.

$x_{1}: \pm 1.5 \%$ (relative error).

\begin{tabular}{|c|c|}
\hline $\begin{array}{l}\text { Components: } \\
\text { (1) Anthracene; } \mathrm{C}_{14} \mathrm{H}_{10} ;[120-12-7] \\
\text { (2) Cyclohexane; } \mathrm{C}_{6} \mathrm{H}_{12} ;[110-82-7] \\
\text { (3) 1,4-Dioxane; } \mathrm{C}_{4} \mathrm{H}_{8} \mathrm{O}_{2} ;[123-91-1] \\
\text { (4) 2-Methyl-1-propanol; } \mathrm{C}_{4} \mathrm{H}_{10} \mathrm{O} \text {; } \\
\text { [78-83-1] }\end{array}$ & $\begin{array}{l}\text { Original Measurements: } \\
{ }^{14} \text { K. J. Pribyla, M. A. Spurgin, I. } \\
\text { Chuca, and W. E. Acree, Jr., J. } \\
\text { Chem. Eng. Data 45, } 971 \text { (2000). }\end{array}$ \\
\hline $\begin{array}{l}\text { Variables: } \\
T / \mathrm{K}=298.15 ; \text { Solvent Composition }\end{array}$ & $\begin{array}{l}\text { Prepared by: } \\
\text { W. E. Acree, Jr. }\end{array}$ \\
\hline
\end{tabular}

Experimental Values

Solubility of anthracene in cyclohexane $+1,4$-dioxane +2 -methyl-1-propanol mixtures $^{\mathrm{a}}$

\begin{tabular}{lcccc}
\hline \hline$x_{3}{ }^{(\mathrm{s})}$ & $x_{4}{ }^{(\mathrm{s})}$ & $x_{3}$ & $x_{4}$ & $x_{1}$ \\
\hline 0.0000 & 0.0000 & 0.0000 & 0.0000 & 0.001553 \\
1.0000 & 0.0000 & 0.9917 & 0.0000 & 0.008329 \\
0.0000 & 1.0000 & 0.0000 & 0.9995 & 0.000470 \\
0.3721 & 0.3373 & 0.3706 & 0.3360 & 0.003942 \\
0.1727 & 0.6946 & 0.1724 & 0.6935 & 0.001615 \\
0.2899 & 0.2726 & 0.2888 & 0.2716 & 0.003642 \\
0.2805 & 0.5009 & 0.2797 & 0.4996 & 0.002688 \\
0.7276 & 0.1055 & 0.7223 & 0.1047 & 0.007223 \\
0.7153 & 0.1935 & 0.7108 & 0.1923 & 0.006340 \\
0.2321 & 0.6752 & 0.2317 & 0.6739 & 0.001885 \\
0.1277 & 0.5149 & 0.1275 & 0.5139 & 0.001858 \\
0.4243 & 0.4828 & 0.4229 & 0.4812 & 0.003416 \\
0.5263 & 0.3845 & 0.5240 & 0.3828 & 0.004381 \\
0.1906 & 0.1188 & 0.1900 & 0.1184 & 0.003291 \\
0.6442 & 0.1900 & 0.6482 & 0.1912 & 0.006236 \\
0.5355 & 0.2505 & 0.5327 & 0.2492 & 0.005275 \\
0.1403 & 0.2208 & 0.1399 & 0.2202 & 0.002622 \\
0.1224 & 0.7002 & 0.1222 & 0.6992 & 0.001383 \\
0.2507 & 0.1208 & 0.2497 & 0.1203 & 0.003816 \\
0.1281 & 0.4287 & 0.1278 & 0.4278 & 0.002072 \\
0.4406 & 0.1124 & 0.4382 & 0.1118 & 0.005454 \\
0.5388 & 0.1185 & 0.5355 & 0.1178 & 0.006045 \\
\hline${ }_{x_{3}}{ }^{(\mathrm{s})}$ : initial mole fraction of component3 in the ternary solvent mixture, $x_{4}{ }^{\left({ }^{()}\right)}$ \\
initial mole fraction of component 4 in the ternary solvent mixture; $x_{1}:$ mole \\
fraction solubility of the solute; $x_{3}:$ mole fraction of component 3 in the \\
quaternary solution; $x_{4}:$ mole fraction of component 4 in the quaternary \\
solution. & & & & \\
& & & &
\end{tabular}

\section{Auxiliary Information}

\section{Method/Apparatus/Procedure:}

Constant-temperature bath, calorimetric thermometer, and an ultraviolet/ visible spectrophotometer.

Ternary solvent mixtures were prepared by mass. Excess solute and solvent were placed in amber glass bottles and allowed to equilibrate for several days at constant temperature. Attainment of equilibrium was verified by several repetitive measurements and by approaching equilibrium from supersaturation. Aliquots of saturated solutions were transferred through a coarse filter into volumetric flasks, weighed and diluted with methanol. Molar concentrations were determined by spectrophotometric measurements at $356 \mathrm{~nm}$.

Source and Purity of Chemicals:

(1) $99+\%$, Aldrich Chemical Company, Milwaukee, WI, USA, was used as received.

(2) $99.9+\%$, HPLC grade, Aldrich Chemical Company.

(3) $99.8 \%$, anhydrous, Aldrich Chemical Company.

(4) $99+\%$, anhydrous, Aldrich Chemical Company.

Components $2-4$ were stored over molecular sieves and distilled shortly before use.

\section{Estimated Error:}

Temperature: $\pm 0.1 \mathrm{~K}$.

$x_{3}{ }^{(\mathrm{s})}: \pm 0.0001$.

$x_{4}{ }^{(\mathrm{s})}: \pm 0.0001$.

$x_{1}: \pm 1.5 \%$ (relative error).

\section{Components:}

(1) Anthracene; $\mathrm{C}_{14} \mathrm{H}_{10}$; [120-12-7]

(2) 2,2,4-Trimethylpentane; $\mathrm{C}_{8} \mathrm{H}_{18}$;

[540-84-1]

(3) 1,4-Dioxane; $\mathrm{C}_{4} \mathrm{H}_{8} \mathrm{O}_{2}$; [123-91-1]

(4) 1-Propanol; $\mathrm{C}_{3} \mathrm{H}_{8} \mathrm{O}$; [71-23-8]

\begin{tabular}{ll}
\hline Variables: & Prepared by: \\
$T / \mathrm{K}=298.15$; Solvent Composition & W. E. Acree, Jr. \\
\hline
\end{tabular}

Experimental Values

Solubility of anthracene in 2,2,4-trimethylpentane + 1,4-dioxane + 1-propanol mixtures $^{\mathrm{a}}$

\begin{tabular}{lcccc}
\hline \hline$x_{3}{ }^{(\mathrm{s})}$ & $x_{4}{ }^{(\mathrm{s})}$ & $x_{3}$ & $x_{4}$ & $x_{1}$ \\
\hline 0.0000 & 0.0000 & 0.0000 & 0.0000 & 0.001074 \\
1.0000 & 0.0000 & 0.9917 & 0.0000 & 0.008329 \\
0.0000 & 1.0000 & 0.0000 & 0.9994 & 0.000591 \\
0.3824 & 0.4276 & 0.3810 & 0.4261 & 0.003572 \\
0.1520 & 0.7702 & 0.1518 & 0.7691 & 0.001487 \\
0.3239 & 0.3608 & 0.3229 & 0.3597 & 0.002991 \\
0.2666 & 0.5766 & 0.2660 & 0.5752 & 0.002373 \\
0.7407 & 0.1434 & 0.7360 & 0.1425 & 0.006409 \\
0.7078 & 0.2375 & 0.7036 & 0.2361 & 0.005865 \\
0.1971 & 0.7474 & 0.1968 & 0.7461 & 0.001725 \\
0.1287 & 0.6383 & 0.1285 & 0.6373 & 0.001523 \\
0.3874 & 0.5574 & 0.3862 & 0.5557 & 0.003102 \\
0.4932 & 0.4514 & 0.4912 & 0.4496 & 0.003966 \\
0.2277 & 0.2584 & 0.2272 & 0.2578 & 0.002334 \\
0.7244 & 0.1926 & 0.7200 & 0.1914 & 0.006102 \\
0.5445 & 0.3125 & 0.5419 & 0.3110 & 0.004755 \\
0.1604 & 0.3299 & 0.1601 & 0.3293 & 0.001899 \\
0.1079 & 0.7861 & 0.1078 & 0.7851 & 0.001257 \\
0.2997 & 0.1747 & 0.2988 & 0.1742 & 0.002859 \\
0.1319 & 0.5565 & 0.1317 & 0.5556 & 0.001628 \\
0.5152 & 0.1585 & 0.5124 & 0.1576 & 0.005463 \\
0.6016 & 0.1545 & 0.5984 & 0.1537 & 0.005330 \\
\hline$x_{3}: 1 n$ & 0.5615 &
\end{tabular}

${ }^{\mathrm{a}} x_{3}{ }^{(\mathrm{s})}$ : initial mole fraction of component 3 in the ternary solvent mixture, $x_{4}{ }^{(\mathrm{s})}$ : initial mole fraction of component 4 in the ternary solvent mixture; $x_{1}$ : mole fraction solubility of the solute; $x_{3}$ : mole fraction of component 3 in the quaternary solution; $x_{4}$ : mole fraction of component 4 in the quaternary solution. 


\section{Auxiliary Information}

\section{Method/Apparatus/Procedure:}

Constant-temperature bath, calorimetric thermometer, and an ultraviolet/ visible spectrophotometer.

Ternary solvent mixtures were prepared by mass. Excess solute and solvent were placed in amber glass bottles and allowed to equilibrate for several days at constant temperature. Attainment of equilibrium was verified by several repetitive measurements and by approaching equilibrium from supersaturation. Aliquots of saturated solutions were transferred through a coarse filter into volumetric flasks, weighed and diluted with methanol. Molar concentrations were determined by spectrophotometric measurements at $356 \mathrm{~nm}$.

\section{Source and Purity of Chemicals:}

(1) $99.9+\%$, Aldrich Chemical Company, Milwaukee, WI, USA, was used as received.

(2) $99.7 \%$, HPLC grade, Aldrich Chemical Company.

(3) $99.8 \%$, anhydrous, Aldrich Chemical Company.

(4) $99+\%$, anhydrous, Aldrich Chemical Company.

Components $2-4$ were stored over molecular sieves and distilled shortly before use.

\section{Estimated Error:}

Temperature: $\pm 0.1 \mathrm{~K}$

$x_{3}{ }^{(\mathrm{s})}: \pm 0.0001$

$x_{4}{ }^{(\mathrm{s})}: \pm 0.0001$

$x_{1}: \pm 1.5 \%$ (relative error).

\section{Components:}

(1) Anthracene; $\mathrm{C}_{14} \mathrm{H}_{10} ;[120-12-7]$

(2) 2,2,4-Trimethylpentane; $\mathrm{C}_{8} \mathrm{H}_{18}$;

[540-84-1]

(3) 1,4-Dioxane; $\mathrm{C}_{4} \mathrm{H}_{8} \mathrm{O}_{2} ;$; [123-91-1]

(4) 2-Propanol; $\mathrm{C}_{3} \mathrm{H}_{8} \mathrm{O}$; [67-63-0]

\begin{tabular}{ll}
\hline Variables: & Prepared by: \\
$T / \mathrm{K}=298.15 ;$ Solvent Composition & W. E. Acree, Jr. \\
\hline
\end{tabular}

\section{Experimental Values}

Solubility of anthracene in 2,2,4-trimethylpentane + 1,4-dioxane + 2-propanol mixtures $^{\mathrm{a}}$

\begin{tabular}{lcccc}
\hline \hline$x_{3}{ }^{(\mathrm{s})}$ & $x_{4}{ }^{(\mathrm{s})}$ & $x_{3}$ & $x_{4}$ & $x_{1}$ \\
\hline 0.0000 & 0.0000 & 0.0000 & 0.0000 & 0.001074 \\
1.0000 & 0.0000 & 0.9917 & 0.0000 & 0.008329 \\
0.0000 & 1.0000 & 0.0000 & 0.9996 & 0.000411 \\
0.3807 & 0.4253 & 0.3795 & 0.4240 & 0.003153 \\
0.1570 & 0.7615 & 0.1568 & 0.7605 & 0.001304 \\
0.3275 & 0.3555 & 0.3266 & 0.3545 & 0.002821 \\
0.2711 & 0.5875 & 0.2705 & 0.5862 & 0.002297 \\
0.7533 & 0.1324 & 0.7484 & 0.1315 & 0.006562 \\
0.7084 & 0.2322 & 0.7042 & 0.2308 & 0.005881 \\
0.1971 & 0.7463 & 0.1968 & 0.7451 & 0.001560 \\
0.1357 & 0.6304 & 0.1355 & 0.6295 & 0.001403 \\
0.3942 & 0.5466 & 0.3930 & 0.5450 & 0.003006 \\
0.4988 & 0.4431 & 0.4969 & 0.4414 & 0.003806
\end{tabular}

$\begin{array}{lllll}0.2314 & 0.2491 & 0.2309 & 0.2485 & 0.002256 \\ 0.7291 & 0.1844 & 0.7246 & 0.1833 & 0.006114 \\ 0.5434 & 0.3132 & 0.5409 & 0.3118 & 0.004520 \\ 0.1595 & 0.3271 & 0.1592 & 0.3265 & 0.001824 \\ 0.1141 & 0.7783 & 0.1140 & 0.7774 & 0.001111 \\ 0.2960 & 0.1822 & 0.2952 & 0.1817 & 0.002706 \\ 0.1323 & 0.5525 & 0.1321 & 0.5517 & 0.001515 \\ 0.5120 & 0.1600 & 0.5097 & 0.1593 & 0.004478 \\ 0.5969 & 0.1526 & 0.5936 & 0.1518 & 0.005466\end{array}$

${ }^{\mathrm{a}} x_{3}{ }^{(\mathrm{s})}$ : initial mole fraction of component 3 in the ternary solvent mixture, $x_{4}{ }^{(\mathrm{s})}$ : initial mole fraction of component 4 in the ternary solvent mixture; $x_{1}$ : mole fraction solubility of the solute; $x_{3}$ : mole fraction of component 3 in the quaternary solution; $x_{4}$ : mole fraction of component 4 in the quaternary solution.

\section{Auxiliary Information}

\section{Method/Apparatus/Procedure:}

Constant-temperature bath, calorimetric thermometer, and an ultraviolet/ visible spectrophotometer.

Ternary solvent mixtures were prepared by mass. Excess solute and solvent were placed in amber glass bottles and allowed to equilibrate for several days at constant temperature. Attainment of equilibrium was verified by several repetitive measurements and by approaching equilibrium from supersaturation. Aliquots of saturated solutions were transferred through a coarse filter into volumetric flasks, weighed and diluted with methanol. Molar concentrations were determined by spectrophotometric measurements at $356 \mathrm{~nm}$.

\section{Source and Purity of Chemicals:}

(1) $99+\%$, Aldrich Chemical Company, Milwaukee, WI, USA, was used as received.

(2) $99.7 \%$, HPLC grade, Aldrich Chemical Company.

(3) $99.8 \%$, anhydrous, Aldrich Chemical Company.

(4) $99+\%$, anhydrous, Aldrich Chemical Company.

Components 2-4 were stored over molecular sieves and distilled shortly before use.

\section{Estimated Error:}

Temperature: $\pm 0.1 \mathrm{~K}$.

$x_{3}{ }^{(\mathrm{s})}: \pm 0.0001$.

$x_{4}{ }^{(\mathrm{s})}: \pm 0.0001$.

$x_{1}: \pm 1.5 \%$ (relative error)

\begin{tabular}{|c|c|}
\hline $\begin{array}{l}\text { Components: } \\
\text { (1) Anthracene; } \mathrm{C}_{14} \mathrm{H}_{10} ;[120-12-7] \\
\text { (2) 2,2,4-Trimethylpentane; } \mathrm{C}_{8} \mathrm{H}_{18} ; \\
\text { [540-84-1] } \\
\text { (3) 1,4-Dioxane; } \mathrm{C}_{4} \mathrm{H}_{8} \mathrm{O}_{2} ;[123-91-1] \\
\text { (4) 1-Butanol; } \mathrm{C}_{4} \mathrm{H}_{10} \mathrm{O} ;[\text { [1-36-3] }\end{array}$ & $\begin{array}{l}\text { Original Measurements: } \\
{ }^{12} \text { K. J. Pribyla, T. T. Van, C. Ezell, } \\
\text { and W. E. Acree, Jr., J. Chem. } \\
\text { Eng. Data 45, } 968 \text { (2000). }\end{array}$ \\
\hline $\begin{array}{l}\text { Variables: } \\
T / \mathrm{K}=298.15 ; \text { Solvent Composition }\end{array}$ & $\begin{array}{l}\text { Prepared by: } \\
\text { W. E. Acree, Jr. }\end{array}$ \\
\hline
\end{tabular}


Experimental Values

Solubility of anthracene in 2,2,4-trimethylpentane +1 ,4-dioxane +1 -butanol mixtures $^{\mathrm{a}}$

\begin{tabular}{lcccc}
\hline \hline$x_{3}{ }^{(\mathrm{s})}$ & $x_{4}{ }^{(\mathrm{s})}$ & $x_{3}$ & $x_{4}$ & $x_{1}$ \\
\hline 0.0000 & 0.0000 & 0.0000 & 0.0000 & 0.001074 \\
1.0000 & 0.0000 & 0.9917 & 0.0000 & 0.008329 \\
0.0000 & 1.0000 & 0.0000 & 0.9992 & 0.000801 \\
0.4126 & 0.3784 & 0.4111 & 0.3770 & 0.003645 \\
0.1800 & 0.7311 & 0.1797 & 0.7298 & 0.001842 \\
0.3476 & 0.3129 & 0.3464 & 0.3118 & 0.003398 \\
0.3025 & 0.5422 & 0.3016 & 0.5406 & 0.002818 \\
0.7721 & 0.1123 & 0.7669 & 0.1115 & 0.006796 \\
0.7336 & 0.2039 & 0.7290 & 0.2026 & 0.006256 \\
0.2219 & 0.7118 & 0.2214 & 0.7103 & 0.002104 \\
0.1431 & 0.5956 & 0.1428 & 0.5945 & 0.001775 \\
0.4396 & 0.4986 & 0.4380 & 0.4967 & 0.003752 \\
0.5378 & 0.3996 & 0.5353 & 0.3978 & 0.004567 \\
0.2378 & 0.2256 & 0.2367 & 0.2246 & 0.002389 \\
0.7535 & 0.1575 & 0.7487 & 0.1565 & 0.006357 \\
0.5751 & 0.2735 & 0.5722 & 0.2721 & 0.005086 \\
0.1659 & 0.2925 & 0.1656 & 0.2919 & 0.002014 \\
0.1281 & 0.7505 & 0.1279 & 0.7493 & 0.001547 \\
0.3010 & 0.1574 & 0.3001 & 0.1569 & 0.002888 \\
0.1510 & 0.5004 & 0.1507 & 0.4995 & 0.001891 \\
0.5303 & 0.1386 & 0.5278 & 0.1379 & 0.004769 \\
0.6200 & 0.1263 & 0.6166 & 0.1256 & 0.005643 \\
\hline${ }_{3}{ }_{3}{ }^{(\mathrm{s})}:$ initial mole fraction of component 3 in the ternary solvent mixture, $x_{4}{ }^{\left({ }^{(s)}\right.}$ : \\
initial mole fraction of component 4 in the ternary solvent mixture; $x_{1}:$ mole \\
fraction solubility of the solute; $x_{3}:$ mole fraction of component 3 in the \\
quaternary solution; $x_{4}:$ mole fraction of component 4 in the quaternary \\
solution. & & & & \\
& & & &
\end{tabular}

\section{Auxiliary Information}

\section{Method/Apparatus/Procedure:}

Constant-temperature bath, calorimetric thermometer, and an ultraviolet/ visible spectrophotometer.

Ternary solvent mixtures were prepared by mass. Excess solute and solvent were placed in amber glass bottles and allowed to equilibrate for several days at constant temperature. Attainment of equilibrium was verified by several repetitive measurements and by approaching equilibrium from supersaturation. Aliquots of saturated solutions were transferred through a coarse filter into volumetric flasks, weighed and diluted with methanol. Molar concentrations were determined by spectrophotometric measurements at $356 \mathrm{~nm}$.

\section{Source and Purity of Chemicals:}

(1) $99+\%$, Aldrich Chemical Company, Milwaukee, WI, USA, was used as received.

(2) $99.7 \%$, HPLC grade, Aldrich Chemical Company.

(3) $99.8 \%$, anhydrous, Aldrich Chemical Company.

(4) $99.8+\%$, HPLC grade, Aldrich Chemical Company.

Components $2-4$ were stored over molecular sieves and distilled shortly before use.

\section{Estimated Error:}

Temperature: $\pm 0.1 \mathrm{~K}$.

$x_{3}^{(\mathrm{s})}: \pm 0.0001$.

$x_{4}{ }^{(\mathrm{s})}: \pm 0.0001$.

$x_{1}: \pm 1.5 \%$ (relative error).

\begin{tabular}{|c|c|}
\hline $\begin{array}{l}\text { Components: } \\
\text { (1) Anthracene; } \mathrm{C}_{14} \mathrm{H}_{10} ;[120-12-7] \\
\text { (2) 2,2,4-Trimethylpentane; } \mathrm{C}_{8} \mathrm{H}_{18} \text {; } \\
\text { [540-84-1] } \\
\text { (3) 1,4-Dioxane; } \mathrm{C}_{4} \mathrm{H}_{8} \mathrm{O}_{2} ;[123-91-1] \\
\text { (4) 2-Butanol; } \mathrm{C}_{4} \mathrm{H}_{10} \mathrm{O} ;[78-92-2]\end{array}$ & $\begin{array}{l}\text { Original Measurements: } \\
{ }^{12} \text { K. J. Pribyla, T. T. Van, C. Ezell, } \\
\text { and W. E. Acree, Jr., J. Chem. } \\
\text { Eng. Data 45, } 968 \text { (2000). }\end{array}$ \\
\hline $\begin{array}{l}\text { Variables: } \\
T / \mathrm{K}=298.15 ; \text { Solvent Composition }\end{array}$ & $\begin{array}{l}\text { Prepared by: } \\
\text { W. E. Acree, Jr. }\end{array}$ \\
\hline
\end{tabular}

Experimental Values

Solubility of anthracene in 2,2,4-trimethylpentane + 1,4-dioxane +2 -butanol mixtures $^{\text {a }}$

\begin{tabular}{lcccc}
\hline \hline$x_{3}(\mathrm{~s})$ & $x_{4}{ }^{(\mathrm{s})}$ & $x_{3}$ & $x_{4}$ & $x_{1}$ \\
\hline 0.0000 & 0.0000 & 0.0000 & 0.0000 & 0.001074 \\
1.0000 & 0.0000 & 0.9917 & 0.0000 & 0.008329 \\
0.0000 & 1.0000 & 0.0000 & 0.9994 & 0.000585 \\
0.4110 & 0.3781 & 0.4095 & 0.3767 & 0.003696 \\
0.1809 & 0.7261 & 0.1806 & 0.7249 & 0.001721 \\
0.3419 & 0.3168 & 0.3408 & 0.3158 & 0.003151 \\
0.3009 & 0.5439 & 0.3001 & 0.5424 & 0.002690 \\
0.7612 & 0.1210 & 0.7560 & 0.1202 & 0.006849 \\
0.7369 & 0.2019 & 0.7321 & 0.2006 & 0.006540 \\
0.2264 & 0.7101 & 0.2259 & 0.7087 & 0.001991 \\
0.1425 & 0.5908 & 0.1423 & 0.5898 & 0.001664 \\
0.4354 & 0.4994 & 0.4338 & 0.4976 & 0.003591 \\
0.5362 & 0.4014 & 0.5337 & 0.3995 & 0.004637 \\
0.2397 & 0.2177 & 0.2391 & 0.2172 & 0.002407 \\
0.7523 & 0.1578 & 0.7472 & 0.1567 & 0.006739 \\
0.5753 & 0.2752 & 0.5723 & 0.2738 & 0.005168 \\
0.1697 & 0.2876 & 0.1694 & 0.2870 & 0.001971 \\
0.1310 & 0.7489 & 0.1308 & 0.7478 & 0.001438 \\
0.3092 & 0.1515 & 0.3083 & 0.1511 & 0.002872 \\
0.1539 & 0.5008 & 0.1536 & 0.4999 & 0.001788 \\
0.5263 & 0.1385 & 0.5238 & 0.1379 & 0.004659 \\
0.6128 & 0.1296 & 0.6093 & 0.1289 & 0.005635 \\
\hline
\end{tabular}

${ }^{a} x_{3}{ }^{(\mathrm{s})}$ : initial mole fraction of component 3 in the ternary solvent mixture, $x_{4}{ }^{(\mathrm{s})}$ : initial mole fraction of component 4 in the ternary solvent mixture; $x_{1}$ : mole fraction solubility of the solute; $x_{3}$ : mole fraction of component 3 in the quaternary solution; $x_{4}$ : mole fraction of component 4 in the quaternary solution.

\section{Method/Apparatus/Procedure:}

Constant-temperature bath, calorimetric thermometer, and an ultraviolet/ visible spectrophotometer.

Ternary solvent mixtures were prepared by mass. Excess solute and solvent were placed in amber glass bottles and allowed to equilibrate for several days at constant temperature. Attainment of equilibrium was verified by several repetitive measurements and by approaching equilibrium from supersaturation. Aliquots of saturated solutions were transferred through a coarse filter into volumetric flasks, weighed and diluted with methanol. Molar concentrations were determined by spectrophotometric measurements at $356 \mathrm{~nm}$. 
Source and Purity of Chemicals:

(1) $99+\%$, Aldrich Chemical Company, Milwaukee, WI, USA, was used as received.

(2) $99.7 \%$, HPLC grade, Aldrich Chemical Company.

(3) $99.8 \%$, anhydrous, Aldrich Chemical Company.

(4) $99+\%$, anhydrous, Aldrich Chemical Company.

Components $2-4$ were stored over molecular sieves and distilled shortly before use.

Estimated Error:

Temperature: $\pm 0.1 \mathrm{~K}$

$x_{3}{ }^{(\mathrm{s})}: \pm 0.0001$.

$x_{4}{ }^{(\mathrm{s})}: \pm 0.0001$.

$x_{1}: \pm 1.5 \%$ (relative error).

\section{Components:}

(1) Anthracene; $\mathrm{C}_{14} \mathrm{H}_{10} ;[120-12-7]$

(2) 2,2,4-Trimethylpentane; $\mathrm{C}_{8} \mathrm{H}_{18}$;

[540-84-1]

(3) 1,4-Dioxane; $\mathrm{C}_{4} \mathrm{H}_{8} \mathrm{O}_{2} ;[123-91-1]$

(4) 2-Methyl-1-propanol; $\mathrm{C}_{4} \mathrm{H}_{10} \mathrm{O}$;

[78-83-1]

\begin{tabular}{ll}
\hline Variables: & Prepared by: \\
$T / \mathrm{K}=298.15$; Solvent Composition & W. E. Acree, Jr. \\
\hline
\end{tabular}

Experimental Values

Solubility of anthracene in 2,2,4-trimethylpentane + 1,4-dioxane + 2-methyl1-propanol mixtures ${ }^{\mathrm{a}}$

\begin{tabular}{lcccc}
\hline \hline$x_{3}{ }^{(\mathrm{s})}$ & $x_{4}{ }^{(\mathrm{s})}$ & $x_{3}$ & $x_{4}$ & $x_{1}$ \\
\hline 0.0000 & 0.0000 & 0.0000 & 0.0000 & 0.001074 \\
1.0000 & 0.0000 & 0.9917 & 0.0000 & 0.008329 \\
0.0000 & 1.0000 & 0.0000 & 0.9995 & 0.000470 \\
0.4093 & 0.3787 & 0.4079 & 0.3774 & 0.003409 \\
0.1800 & 0.7254 & 0.1797 & 0.7244 & 0.001444 \\
0.3446 & 0.3136 & 0.3436 & 0.3127 & 0.002977 \\
0.3053 & 0.5398 & 0.3046 & 0.5385 & 0.002401 \\
0.7692 & 0.1149 & 0.7640 & 0.1141 & 0.006714 \\
0.7352 & 0.2016 & 0.7307 & 0.2004 & 0.006135 \\
0.2201 & 0.6960 & 0.2197 & 0.6948 & 0.001663 \\
0.1583 & 0.5389 & 0.1581 & 0.5381 & 0.001514 \\
0.4370 & 0.5014 & 0.4356 & 0.4998 & 0.003199 \\
0.5390 & 0.3968 & 0.5369 & 0.3952 & 0.003952 \\
0.2394 & 0.2206 & 0.2388 & 0.2201 & 0.002337 \\
0.7509 & 0.1623 & 0.7462 & 0.1613 & 0.006201 \\
0.5763 & 0.2733 & 0.5736 & 0.2720 & 0.004698 \\
0.1731 & 0.2856 & 0.1728 & 0.2851 & 0.001893 \\
0.1281 & 0.7457 & 0.1279 & 0.7448 & 0.001182 \\
0.3013 & 0.1651 & 0.3005 & 0.1647 & 0.002702 \\
0.1618 & 0.4950 & 0.1615 & 0.4942 & 0.001561 \\
0.5260 & 0.1375 & 0.5237 & 0.1369 & 0.004388 \\
0.6187 & 0.1247 & 0.6155 & 0.1240 & 0.005230 \\
\hline$x_{3}(5)$
\end{tabular}

${ }^{\mathrm{a}} x_{3}{ }^{(\mathrm{s})}$ : initial mole fraction of component 3 in the ternary solvent mixture, $x_{4}{ }^{(\mathrm{s})}$ : initial mole fraction of component 4 in the ternary solvent mixture; $x_{1}$ : mole fraction solubility of the solute; $x_{3}$ : mole fraction of component 3 in the quaternary solution; $x_{4}$ : mole fraction of component 4 in the quaternary solution.

\section{Auxiliary Information}

Method/Apparatus/Procedure:

Constant-temperature bath, calorimetric thermometer, and an ultraviolet/ visible spectrophotometer.

Ternary solvent mixtures were prepared by mass. Excess solute and solvent were placed in amber glass bottles and allowed to equilibrate for several days at constant temperature. Attainment of equilibrium was verified by several repetitive measurements and by approaching equilibrium from supersaturation. Aliquots of saturated solutions were transferred through a coarse filter into volumetric flasks, weighed and diluted with methanol. Molar concentrations were determined by spectrophotometric measurements at $356 \mathrm{~nm}$.

Source and Purity of Chemicals:

(1) $99+\%$, Aldrich Chemical Company, Milwaukee, WI, USA, was used as received.

(2) $99.7 \%$, HPLC grade, Aldrich Chemical Company.

(3) $99.8 \%$, anhydrous, Aldrich Chemical Company.

(4) $99+\%$, anhydrous, Aldrich Chemical Company.

Components $2-4$ were stored over molecular sieves and distilled shortly before use.

Estimated Error:

Temperature: $\pm 0.1 \mathrm{~K}$.

$x_{3}{ }^{(\mathrm{s})}: \pm 0.0001$.

$x_{4}{ }^{(\mathrm{s})}: \pm 0.0001$.

$x_{1}: \pm 1.5 \%$ (relative error).

\section{Solubility of Anthracene in Ternary Alkane + Alkane + Alkoxyalcohol Solvent Mixtures}

\subsection{Critical evaluation of experimental solubility data}

Deng and co-workers ${ }^{24}$ measured the solubility of anthracene in ternary cyclohexane + heptane + alkoxyalcohol and cyclohexane $+2,2,4$-trimethylpentane + alkoxyalcohol solvent mixtures at $298.15 \mathrm{~K}$. Two alkoxyalcohol cosolvents, 2ethoxyethanol and 2-butoxyethanol, were studied. There has only been a single experimental determination for the solubility of anthracene in each of the ternary solvent systems studied. The experimental values were evaluated by curvefitting the measured solubility data for the respective subbinary solvent systems to the Combined NIBS/Redlich-Kister equation, and then using Eq. (2) to estimate the mole fraction solubility of anthracene dissolved in ternary alkane + alkane + alkoxyalcohol solvent mixtures.

The calculated $S_{23, i}$ parameters for all of the contributing binary alkane + alkane and alkane + alcohol solvent systems studied are summarized in Table 1, along with the average absolute deviation between the experimental and back-calculated mole fraction solubilities. The predictive ability of Eq. (2) is given in Table 6 for the four ternary alkane + alkane + alcohol solvent systems. Examination of the numerical entries in the last column of Table 6 reveals that Eq. (2) does provide a 
TABLE 6. Summarized comparison between experimental solubilities of anthracene in ternary alkane + alkane + alkoxyalcohol solvent mixtures and predicted values based on Eq. (2)

\begin{tabular}{lc}
\hline \hline Ternary solvent mixture & $\%$ Dev. ${ }^{\mathrm{a}}$ \\
\hline Heptane + cyclohexane + 2-ethoxyethanol & 1.30 \\
Cyclohexane + 2,2,4-trimethylpentane + 2-ethoxyethanol & 1.53 \\
Heptane + cyclohexane + 2-butoxyethanol & 1.26 \\
Cyclohexane + 2,2,4-trimethylpentane + 2-butoxyethanol & 1.60 \\
\hline
\end{tabular}

${ }^{\mathrm{a}} \operatorname{Dev}(\%)=(100 / N) \Sigma\left|\left[x_{1}{ }^{\mathrm{exp}}-x_{1}{ }^{\mathrm{cal}}\right] / x_{1}{ }^{\mathrm{exp}}\right|$.

very accurate mathematical representation for how the measured mole fraction solubilities vary with binary solvent composition. Average percent deviations range from $1.3 \%$ to $1.6 \%$, suggesting that there are no obvious outliers in an individual ternary solvent data set. None of the experimental data points was flagged as an outlier.

The experimental anthracene solubilities for ternary alkane + alkane + alkoxyalcohol solvent mixtures are reported in Secs. 5.2 and 5.3.

\subsection{Anthracene solubility data in ternary alkane + alkane + 2-ethoxyethanol solvent mixtures}

\begin{tabular}{ll}
\hline \hline Components: & Original Measurements: \\
(1) Anthracene; $\mathrm{C}_{14} \mathrm{H}_{10} ;[120-12-7]$ & ${ }^{24}$ T. Deng, S. D. Childress, K. M. \\
(2) Heptane; $\mathrm{C}_{7} \mathrm{H}_{16} ;[142-82-5]$ & De Fina, C. E. Hernández, L. E. \\
(3) Cyclohexane; $\mathrm{C}_{6} \mathrm{H}_{12} ;[110-82-7]$ & Roy, T. L. Sharp, and W. E. Acree, \\
(4) 2-Ethoxyethanol; $\mathrm{C}_{4} \mathrm{H}_{10} \mathrm{O}_{2} ;$ & Jr., J. Chem. Eng. Data 44, 357 \\
{$[110-80-5]$} & $(1999)$. \\
\hline Variables: & Prepared by: \\
$T / \mathrm{K}=298.15 ;$ Solvent Composition & W. E. Acree, Jr. \\
\hline
\end{tabular}

\section{Experimental Values}

Solubility of anthracene in heptane + cyclohexane +2 -ethoxyethanol mixtures ${ }^{\mathrm{a}}$

\begin{tabular}{lcccc}
\hline \hline$x_{3}^{(\mathrm{s})}$ & $x_{4}{ }^{(\mathrm{s})}$ & $x_{3}$ & $x_{4}$ & $x_{1}$ \\
\hline 0.0000 & 0.0000 & 0.0000 & 0.0000 & 0.001571 \\
1.0000 & 0.0000 & 0.9984 & 0.0000 & 0.001553 \\
0.0000 & 1.0000 & 0.0000 & 0.9971 & 0.002921 \\
0.3505 & 0.3938 & 0.3495 & 0.3927 & 0.002892 \\
0.7122 & 0.1742 & 0.7105 & 0.1738 & 0.002319 \\
0.2773 & 0.3113 & 0.2766 & 0.3105 & 0.002617 \\
0.5167 & 0.2919 & 0.5154 & 0.2911 & 0.002589 \\
0.0982 & 0.7590 & 0.0979 & 0.7565 & 0.003310 \\
0.1898 & 0.7391 & 0.1892 & 0.7366 & 0.003337 \\
0.7008 & 0.2226 & 0.6991 & 0.2221 & 0.002437 \\
0.5498 & 0.1275 & 0.5486 & 0.1272 & 0.002110 \\
0.4905 & 0.4380 & 0.4890 & 0.4367 & 0.003064
\end{tabular}

\begin{tabular}{lllll}
0.3868 & 0.5417 & 0.3855 & 0.5399 & 0.003242 \\
0.1774 & 0.2028 & 0.1770 & 0.2023 & 0.002297 \\
0.1408 & 0.7536 & 0.1403 & 0.7511 & 0.003301 \\
0.2558 & 0.5575 & 0.2550 & 0.5557 & 0.003142 \\
0.2412 & 0.1366 & 0.2407 & 0.1363 & 0.002072 \\
0.7309 & 0.1170 & 0.7294 & 0.1168 & 0.002108 \\
0.1167 & 0.2665 & 0.1164 & 0.2659 & 0.002402 \\
0.4481 & 0.1358 & 0.4471 & 0.1355 & 0.002131 \\
0.1123 & 0.4867 & 0.1120 & 0.4853 & 0.002894 \\
0.1085 & 0.5839 & 0.1082 & 0.5821 & 0.003140 \\
\hline${ }^{a} x_{3}{ }^{(s)}:$ initial mole fraction of component 3 in the ternary solvent mixture, $x_{4}{ }^{\left({ }^{(s)}\right.}$ : \\
initial mole fraction of component 4 in the ternary solvent mixture; $x_{1}:$ mole \\
fraction solubility of the solute; $x_{3}:$ mole fraction of component 3 in the \\
quaternary solution; $x_{4}:$ mole fraction of component 4 in the quaternary \\
solution.
\end{tabular}

\section{Auxiliary Information}

\begin{abstract}
Method/Apparatus/Procedure:
Constant-temperature bath, calorimetric thermometer, and an ultraviolet/ visible spectrophotometer.

Ternary solvent mixtures were prepared by mass. Excess solute and solvent were placed in amber glass bottles and allowed to equilibrate for several days at constant temperature. Attainment of equilibrium was verified by several repetitive measurements and by approaching equilibrium from supersaturation. Aliquots of saturated solutions were transferred through a coarse filter into volumetric flasks, weighed and diluted with methanol. Molar concentrations were determined by spectrophotometric measurements at $356 \mathrm{~nm}$.
\end{abstract}

Source and Purity of Chemicals:

(1) $99+\%$, Acros Chemical Company, USA, recrystallized three times from acetone.

(2) 99\%, HPLC grade, Aldrich Chemical Company, Milwaukee, WI, USA.

(3) $99.9+\%$, HPLC grade, Aldrich Chemical Company.

(4) $99 \%$, Aldrich Chemical Company.

Components 2-4 were stored over molecular sieves and distilled shortly before use.

Estimated Error:

Temperature: $\pm 0.1 \mathrm{~K}$.

$x_{3}{ }^{(\mathrm{s})}: \pm 0.0001$.

$x_{4}{ }^{(\mathrm{s})}: \pm 0.0001$.

$x_{1}: \pm 1.7 \%$ (relative error).

\begin{tabular}{|c|c|}
\hline $\begin{array}{l}\text { Components: } \\
\text { (1) Anthracene; } \mathrm{C}_{14} \mathrm{H}_{10} ;[120-12-7] \\
\text { (2) } 2,2,4-\text { Trimethylpentane; } \mathrm{C}_{8} \mathrm{H}_{18} \text {; } \\
\text { [540-84-1] } \\
\text { (3) Cyclohexane; } \mathrm{C}_{6} \mathrm{H}_{12} ;[110-82-7] \\
\text { (4) 2-Ethoxyethanol; } \mathrm{C}_{4} \mathrm{H}_{10} \mathrm{O}_{2} ; \\
\text { [110-80-5] }\end{array}$ & $\begin{array}{l}\text { Original Measurements: } \\
{ }^{24} \text { T. Deng, S. D. Childress, K. M. } \\
\text { De Fina, C. E. Hernández, L. E. } \\
\text { Roy, T. L. Sharp, and W. E. Acree, } \\
\text { Jr., J. Chem. Eng. Data 44, } 357 \\
\text { (1999). }\end{array}$ \\
\hline $\begin{array}{l}\text { Variables: } \\
T / \mathrm{K}=298.15 \text {; Solvent Composition }\end{array}$ & $\begin{array}{l}\text { Prepared by: } \\
\text { W. E. Acree, Jr. }\end{array}$ \\
\hline
\end{tabular}


Experimental Values

Solubility of anthracene in 2,2,4-trimethylpentane + cyclohexane +2 ethoxyethanol mixtures ${ }^{\mathrm{a}}$

\begin{tabular}{lcccc}
\hline \hline$x_{3}{ }^{(\mathrm{s})}$ & $x_{4}{ }^{(\mathrm{s})}$ & $x_{3}$ & $x_{4}$ & $x_{1}$ \\
\hline 0.0000 & 0.0000 & 0.0000 & 0.0000 & 0.001074 \\
1.0000 & 0.0000 & 0.9984 & 0.0000 & 0.001553 \\
0.0000 & 1.0000 & 0.0000 & 0.9971 & 0.002921 \\
0.3604 & 0.4026 & 0.3595 & 0.4015 & 0.002614 \\
0.7223 & 0.1747 & 0.7207 & 0.1743 & 0.002240 \\
0.2930 & 0.3268 & 0.2923 & 0.3261 & 0.002262 \\
0.5293 & 0.2976 & 0.5280 & 0.2069 & 0.002470 \\
0.1010 & 0.7683 & 0.1007 & 0.7659 & 0.003173 \\
0.1890 & 0.7498 & 0.1884 & 0.7473 & 0.003333 \\
0.7079 & 0.2269 & 0.7062 & 0.2263 & 0.002436 \\
0.5702 & 0.1315 & 0.5619 & 0.1313 & 0.001846 \\
0.4944 & 0.4415 & 0.4929 & 0.4402 & 0.002953 \\
0.3923 & 0.5460 & 0.3911 & 0.5443 & 0.003110 \\
0.1914 & 0.2183 & 0.1911 & 0.2179 & 0.001821 \\
0.1461 & 0.7585 & 0.1456 & 0.7561 & 0.003190 \\
0.2574 & 0.5739 & 0.2566 & 0.5722 & 0.002963 \\
0.2619 & 0.1402 & 0.2613 & 0.1400 & 0.001613 \\
0.7428 & 0.1182 & 0.7414 & 0.1180 & 0.001946 \\
0.1289 & 0.2889 & 0.1286 & 0.2883 & 0.001947 \\
0.4810 & 0.1297 & 0.4801 & 0.1295 & 0.001768 \\
0.1169 & 0.5128 & 0.1166 & 0.5115 & 0.002549 \\
0.1080 & 0.6075 & 0.1077 & 0.6058 & 0.002739 \\
\hline $\mathrm{a}_{x^{(\mathrm{s})}}$ initial mole fraction of & & &
\end{tabular}

initial mole fraction of component 4 in the ternary solvent mixture; $x_{1}$ : mole fraction solubility of the solute; $x_{3}$ : mole fraction of component 3 in the quaternary solution; $x_{4}$ : mole fraction of component 4 in the quaternary solution.

\subsection{Anthracene solubility data in ternary alkane + alkane + 2-butoxyethanol solvent mixtures}

\begin{tabular}{ll}
\hline \hline Components: & Original Measurements: \\
(1) Anthracene; $\mathrm{C}_{14} \mathrm{H}_{10} ;[120-12-7]$ & ${ }^{24}$ T. Deng, S. D. Childress, K. M. \\
(2) Heptane; $\mathrm{C}_{7} \mathrm{H}_{16} ;[142-82-5]$ & De Fina, C. E. Hernández, L. E. \\
(3) Cyclohexane; $\mathrm{C}_{6} \mathrm{H}_{12} ;[110-82-7]$ & Roy, T. L. Sharp, and W. E. Acree, \\
(4) 2-Butoxyethanol; $\mathrm{C}_{6} \mathrm{H}_{14} \mathrm{O}_{2} ;$ & Jr., J. Chem. Eng. Data 44, 357 \\
{$[111-76-2]$} & $(1999)$. \\
\hline Variables: & Prepared by: \\
$T / \mathrm{K}=298.15 ;$ Solvent Composition & W. E. Acree, Jr. \\
\hline
\end{tabular}

Experimental Values

Solubility of anthracene in heptane + cyclohexane +2 -butoxyethanol mixtures

\begin{tabular}{|c|c|c|c|c|}
\hline$x_{3}{ }^{(s)}$ & $x_{4}{ }^{(s)}$ & $x_{3}$ & $x_{4}$ & $x_{1}$ \\
\hline 0.0000 & 0.0000 & 0.0000 & 0.0000 & 0.001571 \\
\hline 1.0000 & 0.0000 & 0.9984 & 0.0000 & 0.001553 \\
\hline 0.0000 & 1.0000 & 0.0000 & 0.9962 & 0.003785 \\
\hline 0.3877 & 0.3226 & 0.3867 & 0.3217 & 0.002669 \\
\hline 0.7450 & 0.1332 & 0.7433 & 0.1329 & 0.002227 \\
\hline 0.3028 & 0.2521 & 0.3021 & 0.2515 & 0.002429 \\
\hline 0.5579 & 0.2324 & 0.5565 & 0.2318 & 0.002487 \\
\hline 0.1207 & 0.7006 & 0.1203 & 0.6982 & 0.003491 \\
\hline 0.2368 & 0.6783 & 0.2360 & 0.6760 & 0.003414 \\
\hline 0.7492 & 0.1750 & 0.7474 & 0.1746 & 0.002373 \\
\hline 0.5734 & 0.0922 & 0.5722 & 0.0920 & 0.002019 \\
\hline 0.5559 & 0.3636 & 0.5543 & 0.3625 & 0.002903 \\
\hline 0.4525 & 0.4648 & 0.4511 & 0.4634 & 0.003026 \\
\hline 0.1889 & 0.1572 & 0.1885 & 0.1569 & 0.002150 \\
\hline 0.1745 & 0.6915 & 0.1739 & 0.6891 & 0.003417 \\
\hline 0.2944 & 0.4873 & 0.2935 & 0.4859 & 0.003064 \\
\hline 0.2485 & 0.1052 & 0.2480 & 0.1050 & 0.002015 \\
\hline 0.7553 & 0.0890 & 0.7538 & 0.0888 & 0.002029 \\
\hline 0.1302 & 0.2089 & 0.1299 & 0.2084 & 0.002317 \\
\hline 0.4699 & 0.0966 & 0.4690 & 0.0964 & 0.001975 \\
\hline 0.1287 & 0.4116 & 0.1283 & 0.4104 & 0.002882 \\
\hline 0.1243 & 0.5109 & 0.1239 & 0.5093 & 0.003068 \\
\hline
\end{tabular}

${ }^{\mathrm{a}} x_{3}{ }^{(\mathrm{s})}$ : initial mole fraction of component 3 in the ternary solvent mixture, $x_{4}{ }^{(\mathrm{s})}$ : initial mole fraction of component 4 in the ternary solvent mixture; $x_{1}$ : mole fraction solubility of the solute; $x_{3}$ : mole fraction of component 3 in the quaternary solution; $x_{4}$ : mole fraction of component 4 in the quaternary solution.

\section{Method/Apparatus/Procedure: \\ Constant-temperature bath, calorimetric thermometer, and an ultraviolet/ visible spectrophotometer. \\ Ternary solvent mixtures were prepared by mass. Excess solute and solvent were placed in amber glass bottles and allowed to equilibrate for several days a repetitive measurements and by approaching equilibrium from supersaturation. Aliquots of saturated solutions were transferred through a coarse filter into volumetric flasks, weighed and diluted with methanol. Molar concentrations were determined by spectrophotometric measurements at $356 \mathrm{~nm}$.}

\section{Source and Purity of Chemicals:}

(1) $99+\%$, Acros Chemical Company, USA, recrystallized three times from acetone.

(2) $99.7+\%$, HPLC grade, Aldrich Chemical Company, Milwaukee, WI, USA.

(3) $99.9+\%$, HPLC grade, Aldrich Chemical Company.

(4) $99 \%$, Aldrich Chemical Company.

Components $2-4$ were stored over molecular sieves and distilled shortly before use.

\section{Estimated Error:}

Temperature: $\pm 0.1 \mathrm{~K}$

$x_{3}^{(\mathrm{s})}: \pm 0.0001$.

$x_{4}{ }^{(\mathrm{s})}: \pm 0.0001$

$x_{1}: \pm 1.7 \%$ (relative error)

\section{Auxiliary Information}

\section{Method/Apparatus/Procedure:}

Constant-temperature bath, calorimetric thermometer, and an ultraviolet/ visible spectrophotometer.

Ternary solvent mixtures were prepared by mass. Excess solute and solvent were placed in amber glass bottles and allowed to equilibrate for several days at constant temperature. Attainment of equilibrium was verified by several repetitive measurements and by approaching equilibrium from supersaturation. Aliquots of saturated solutions were transferred through a coarse filter into volumetric flasks, weighed and diluted with methanol. Molar concentrations were determined by spectrophotometric measurements at $356 \mathrm{~nm}$. 
Source and Purity of Chemicals:

(1) $99+\%$, Acros Chemical Company, USA, recrystallized three times from acetone.

(2) $99 \%$, HPLC grade, Aldrich Chemical Company, Milwaukee, WI, USA.

(3) $99.9+\%$, HPLC grade, Aldrich Chemical Company.

(4) $99+\%$, Acros Chemical Company.

Components $2-4$ were stored over molecular sieves and distilled shortly before use.

Estimated Error:

Temperature: $\pm 0.1 \mathrm{~K}$

$x_{3}{ }^{(s)}: \pm 0.0001$.

$x_{4}^{(\mathrm{s})}: \pm 0.0001$.

$x_{1}: \pm 1.7 \%$ (relative error).

\section{Components:}

(1) Anthracene; $\mathrm{C}_{14} \mathrm{H}_{10} ;[120-12-7]$

(2) 2,2,4-Trimethylpentane; $\mathrm{C}_{8} \mathrm{H}_{18}$;

[540-84-1]

(3) Cyclohexane; $\mathrm{C}_{6} \mathrm{H}_{12} ;[110-82-7]$

(4) 2-Butoxyethanol; $\mathrm{C}_{6} \mathrm{H}_{14} \mathrm{O}_{2}$;

[111-76-2]

\begin{tabular}{ll}
\hline Variables: & Prepared by: \\
$T / \mathrm{K}=298.15 ;$ Solvent Composition & W. E. Acree, Jr. \\
\hline
\end{tabular}

Experimental Values

Solubility of anthracene in 2,2,4-trimethylpentane + cyclohexane +2 butoxyethanol mixtures ${ }^{\mathrm{a}}$

\begin{tabular}{lcccc}
\hline \hline$x_{3}{ }^{(\mathrm{s})}$ & $x_{4}{ }^{(\mathrm{s})}$ & $x_{3}$ & $x_{4}$ & $x_{1}$ \\
\hline 0.0000 & 0.0000 & 0.0000 & 0.0000 & 0.001074 \\
1.0000 & 0.0000 & 0.9984 & 0.0000 & 0.001553 \\
0.0000 & 1.0000 & 0.0000 & 0.9962 & 0.003785 \\
0.4025 & 0.3300 & 0.4015 & 0.3292 & 0.002415 \\
0.7585 & 0.1357 & 0.7569 & 0.1354 & 0.002099 \\
0.3146 & 0.2686 & 0.3139 & 0.2680 & 0.002113 \\
0.5732 & 0.2386 & 0.5719 & 0.2380 & 0.002314 \\
0.1220 & 0.7169 & 0.1216 & 0.7146 & 0.003227 \\
0.2348 & 0.6873 & 0.2340 & 0.6850 & 0.003313 \\
0.7500 & 0.1766 & 0.7483 & 0.1762 & 0.002304 \\
0.5913 & 0.0997 & 0.5903 & 0.0995 & 0.001739 \\
0.5568 & 0.3692 & 0.5552 & 0.3682 & 0.002813 \\
0.4506 & 0.4706 & 0.4492 & 0.4692 & 0.003019 \\
0.2036 & 0.1718 & 0.2032 & 0.1715 & 0.001726 \\
0.1814 & 0.6986 & 0.1808 & 0.6963 & 0.003283 \\
0.3059 & 0.4965 & 0.3050 & 0.4951 & 0.002823 \\
0.2664 & 0.1153 & 0.2660 & 0.1151 & 0.001601 \\
0.7656 & 0.0935 & 0.7642 & 0.0933 & 0.001863 \\
0.1388 & 0.2308 & 0.1385 & 0.2304 & 0.001860 \\
0.4913 & 0.1027 & 0.4905 & 0.1025 & 0.001702 \\
0.1323 & 0.4376 & 0.1320 & 0.4365 & 0.002436 \\
0.1280 & 0.5343 & 0.1277 & 0.5328 & 0.002729 \\
\hline${ }^{a} x_{3}{ }^{(s)}:$ initial mole fraction of component 3 in the ternary solvent mixture, $x_{4}{ }^{(\mathrm{s})}$ : \\
initial mole fraction of component 4 in the ternary solvent mixture; $x_{1}:$ mole \\
fraction solubility of the solute; $x_{3}:$ mole fraction of component 3 in the \\
quaternary solution; $x_{4}:$ mole fraction of component 4 in the quaternary \\
solution. & & & & \\
& & & &
\end{tabular}

\section{Auxiliary Information}

Method/Apparatus/Procedure:

Constant-temperature bath, calorimetric thermometer, and an ultraviolet/ visible spectrophotometer.

Ternary solvent mixtures were prepared by mass. Excess solute and solvent were placed in amber glass bottles and allowed to equilibrate for several days at constant temperature. Attainment of equilibrium was verified by several repetitive measurements and by approaching equilibrium from supersaturation. Aliquots of saturated solutions were transferred through a coarse filter into volumetric flasks, weighed and diluted with methanol. Molar concentrations were determined by spectrophotometric measurements at $356 \mathrm{~nm}$.

Source and Purity of Chemicals:

(1) $99+\%$, Acros Chemical Company, USA, recrystallized three times from acetone.

(2) $99 \%$, HPLC grade, Aldrich Chemical Company, Milwaukee, WI, USA.

(3) $99.9+\%$, HPLC grade, Aldrich Chemical Company.

(4) $99+\%$, Acros Chemical Company.

Components $2-4$ were stored over molecular sieves and distilled shortly before use.

Estimated Error:

Temperature: $\pm 0.1 \mathrm{~K}$.

$x_{3}{ }^{(\mathrm{s})}: \pm 0.0001$.

$x_{4}^{(s)}: \pm 0.0001$.

$x_{1}: \pm 1.7 \%$ (relative error)

\section{Solubility of Anthracene in Ternary Alkane + Alcohol + Alkoxyalcohol Solvent Mixtures}

\subsection{Critical evaluation of experimental solubility data}

Deng and co-workers ${ }^{25}$ determined the solubility of anthracene in ternary solvent mixtures containing heptane + propanol + 2-butoxyethanol and cyclohexane + propanol + 2-butoxyethanol at $298.15 \mathrm{~K}$. There has only been a single experimental determination for the solubility of anthracene in each of the ternary solvent systems studied. In the absence of replicate independent measurements, the experimental values were evaluated by curve-fitting the measured solubility data in the sub-binary solvent systems, and then using Eq. (2) to estimate the mole fraction solubility of anthracene dissolved in ternary alkane + alcohol + alkoxyalcohol solvent mixtures.

The calculated $S_{23, i}$ parameters for all of the contributing binary alkane + alcohol, alkane + alkoxyalcohol, and alcohol + alkoxyalcohol solvent systems studied are summarized in Table 1, along with the average absolute deviation between the experimental and back-calculated mole fraction solubilities. The predictive ability of Eq. (2) is given in Table 7 for the four ternary alkane + alcohol + alkoxyalcohol solvent systems. Examination of the numerical entries in the last column of Table 7 reveals that Eq. (2) does provide a very accurate 
TABLE 7. Summarized comparison between experimental solubilities of anthracene in ternary alkane + alcohol + alkoxyalcohol solvent mixtures and predicted values based on Eq. (2)

\begin{tabular}{lc}
\hline \hline Ternary solvent mixture & $\%$ Dev. \\
\hline Heptane + 1-propanol + 2-butoxyethanol & 1.46 \\
Heptane + 2-propanol + 2-butoxyethanol & 1.25 \\
Cyclohexane + 1-propanol + 2-butoxyethanol & 1.63 \\
Cyclohexane + 2-propanol + 2-butoxyethanol & 1.58 \\
\hline${ }^{a}$ Dev $(\%)=(100 / N) \sum\left|\left[x^{\exp }-x^{\text {cal }}\right] / x^{\exp }\right|$
\end{tabular}

${ }^{\mathrm{a}} \operatorname{Dev}(\%)=(100 / N) \sum\left|\left[x_{1}{ }^{\text {exp }}-x_{1}{ }^{\text {cal }}\right] / x_{1}{ }^{\text {exp }}\right|$.

mathematical representation for how the measured mole fraction solubilities vary with binary solvent composition. Average percent deviations range from $1.3 \%$ to $1.6 \%$, suggesting that there are no obvious outliers in an individual ternary solvent data set. None of the experimental data points was flagged as an outlier.

The experimental anthracene solubility data for ternary heptane + propanol + 2-butoxyethanol and cyclohexane + propanol +2 -butoxyethanol solvent mixtures are given in Sec. 6.2.

\subsection{Anthracene solubility data in ternary alkane + alcohol + 2-butoxyethanol solvent mixtures}

\begin{tabular}{|c|c|}
\hline $\begin{array}{l}\text { Components: } \\
\text { (1) Anthracene; } \mathrm{C}_{14} \mathrm{H}_{10} ;[120-12-7] \\
\text { (2) Heptane; } \mathrm{C}_{7} \mathrm{H}_{16} ;[142-82-5] \\
\text { (3) 1-Propanol; } \mathrm{C}_{3} \mathrm{H}_{8} \mathrm{O} ;[71-23-8] \\
\text { (4) 2-Butoxyethanol; } \mathrm{C}_{6} \mathrm{H}_{14} \mathrm{O}_{2} ; \\
\text { [111-76-2] }\end{array}$ & $\begin{array}{l}\text { Original Measurements: } \\
{ }^{25} \text { T. Deng, S. Horiuchi, L. E. Roy, } \\
\text { and W. E. Acree, Jr., J. Chem. } \\
\text { Eng. Data 44, } 258 \text { (1999). }\end{array}$ \\
\hline $\begin{array}{l}\text { Variables: } \\
T / \mathrm{K}=298.15 ; \text { Solvent Composition }\end{array}$ & $\begin{array}{l}\text { Prepared by: } \\
\text { W. E. Acree, Jr. }\end{array}$ \\
\hline
\end{tabular}

Experimental Values

Solubility of anthracene in heptane +1 -propanol +2 -butoxyethanol mixtures ${ }^{\mathrm{a}}$

\begin{tabular}{lcccc}
\hline \hline$x_{2}{ }^{(\mathrm{s})}$ & $x_{4}{ }^{(\mathrm{s})}$ & $x_{2}$ & $x_{4}$ & $x_{1}$ \\
\hline 0.0000 & 0.0000 & 0.0000 & 0.0000 & 0.000591 \\
1.0000 & 0.0000 & 0.9984 & 0.0000 & 0.001571 \\
0.0000 & 1.0000 & 0.0000 & 0.9962 & 0.003785 \\
0.2433 & 0.2770 & 0.2420 & 0.2765 & 0.001844 \\
0.5948 & 0.1463 & 0.5937 & 0.1460 & 0.001931 \\
0.1644 & 0.1856 & 0.1642 & 0.1853 & 0.001426 \\
0.3881 & 0.2274 & 0.3874 & 0.2270 & 0.001932 \\
0.0798 & 0.6136 & 0.0796 & 0.6120 & 0.002601 \\
0.1688 & 0.6615 & 0.1683 & 0.6595 & 0.003033 \\
0.6251 & 0.2017 & 0.6237 & 0.2013 & 0.002182 \\
0.3568 & 0.0822 & 0.3563 & 0.0821 & 0.001378 \\
0.4377 & 0.3921 & 0.4366 & 0.3911 & 0.002591 \\
0.3442 & 0.4854 & 0.3432 & 0.4841 & 0.002769
\end{tabular}

$\begin{array}{lllll}0.0882 & 0.1003 & 0.0881 & 0.1002 & 0.001013 \\ 0.1221 & 0.6392 & 0.1217 & 0.6374 & 0.002888 \\ 0.1908 & 0.4298 & 0.1904 & 0.4288 & 0.002221 \\ 0.1174 & 0.0687 & 0.1173 & 0.0686 & 0.000945 \\ 0.5804 & 0.0962 & 0.5794 & 0.0960 & 0.001727 \\ 0.0594 & 0.1336 & 0.0953 & 0.1335 & 0.001061 \\ 0.2680 & 0.0768 & 0.2677 & 0.0767 & 0.001261 \\ 0.0670 & 0.2925 & 0.0669 & 0.2921 & 0.001508 \\ 0.0697 & 0.3893 & 0.0696 & 0.3886 & 0.001845\end{array}$

${ }^{\mathrm{a}} x_{2}{ }^{(\mathrm{s})}$ : initial mole fraction of component 2 in the ternary solvent mixture, $x_{4}{ }^{(\mathrm{s})}$ : initial mole fraction of component 4 in the ternary solvent mixture; $x_{1}$ : mole fraction solubility of the solute; $x_{2}$ : mole fraction of component 2 in the quaternary solution; $x_{4}$ : mole fraction of component 4 in the quaternary solution.

\section{Auxiliary Information}

\section{Method/Apparatus/Procedure:}

Constant-temperature bath, calorimetric thermometer, and an ultraviolet/ visible spectrophotometer.

Ternary solvent mixtures were prepared by mass. Excess solute and solvent were placed in amber glass bottles and allowed to equilibrate for several days at constant temperature. Attainment of equilibrium was verified by several repetitive measurements and by approaching equilibrium from supersaturation. Aliquots of saturated solutions were transferred through a coarse filter into volumetric flasks, weighed and diluted with methanol. Molar concentrations were determined by spectrophotometric measurements at $356 \mathrm{~nm}$.

Source and Purity of Chemicals:

(1) $99+\%$, Acros Chemical Company, USA, recrystallized three times from acetone.

(2) $99 \%$, HPLC grade, Aldrich Chemical Company, Milwaukee, WI, USA.

(3) $99+\%$, anhydrous, Aldrich Chemical Company.

(4) $99+\%$, Acros Chemical Company.

Components 2-4 were stored over molecular sieves and distilled shortly before use.

\section{Estimated Error:}

Temperature: $\pm 0.1 \mathrm{~K}$.

$x_{2}{ }^{(\mathrm{s})}: \pm 0.0001$.

$x_{4}{ }^{(s)}: \pm 0.0001$.

$x_{1}: \pm 1.5 \%$ (relative error)

\begin{tabular}{|c|c|}
\hline $\begin{array}{l}\text { Components: } \\
\text { (1) Anthracene; } \mathrm{C}_{14} \mathrm{H}_{10} ;[120-12-7] \\
\text { (2) Heptane; } \mathrm{C}_{7} \mathrm{H}_{16} ;[142-82-5] \\
\text { (3) 2-Propanol; } \mathrm{C}_{3} \mathrm{H}_{8} \mathrm{O} ;[67-63-0] \\
\text { (4) 2-Butoxyethanol; } \mathrm{C}_{6} \mathrm{H}_{14} \mathrm{O}_{2} ; \\
\text { [111-76-2] }\end{array}$ & $\begin{array}{l}\text { Original Measurements: } \\
{ }^{25} \text { T. Deng, S. Horiuchi, L. E. Roy, } \\
\text { and W. E. Acree, Jr., J. Chem. } \\
\text { Eng. Data 44, } 258 \text { (1999). }\end{array}$ \\
\hline $\begin{array}{l}\text { Variables: } \\
T / \mathrm{K}=298.15 ; \text { Solvent Composition }\end{array}$ & $\begin{array}{l}\text { Prepared by: } \\
\text { W. E. Acree, Jr. }\end{array}$ \\
\hline
\end{tabular}


Experimental Values

Solubility of anthracene in heptane +2 -propanol +2 -butoxyethanol mixtures ${ }^{\mathrm{a}}$

\begin{tabular}{|c|c|c|c|c|}
\hline$x_{2}{ }^{(\mathrm{s})}$ & $x_{4}{ }^{(\mathrm{s})}$ & $x_{2}$ & $x_{4}$ & $x_{1}$ \\
\hline 0.0000 & 0.0000 & 0.0000 & 0.0000 & 0.000411 \\
\hline 1.0000 & 0.0000 & 0.9984 & 0.0000 & 0.001571 \\
\hline 0.0000 & 1.0000 & 0.0000 & 0.9962 & 0.003785 \\
\hline 0.2506 & 0.2779 & 0.2502 & 0.2774 & 0.001699 \\
\hline 0.6020 & 0.1451 & 0.6003 & 0.1448 & 0.001867 \\
\hline 0.1686 & 0.1894 & 0.1684 & 0.1892 & 0.001255 \\
\hline 0.3978 & 0.2240 & 0.3971 & 0.2236 & 0.001817 \\
\hline 0.0806 & 0.6184 & 0.0804 & 0.6168 & 0.002595 \\
\hline 0.1708 & 0.6671 & 0.1703 & 0.6651 & 0.002968 \\
\hline 0.6202 & 0.2021 & 0.6189 & 0.2017 & 0.002087 \\
\hline 0.3594 & 0.0818 & 0.3590 & 0.0817 & 0.001217 \\
\hline 0.4316 & 0.3921 & 0.4305 & 0.3911 & 0.002482 \\
\hline 0.3448 & 0.4800 & 0.3439 & 0.4787 & 0.002665 \\
\hline 0.0933 & 0.1035 & 0.0932 & 0.1034 & 0.000853 \\
\hline 0.1245 & 0.6409 & 0.1242 & 0.6391 & 0.002783 \\
\hline 0.1941 & 0.4340 & 0.1937 & 0.4331 & 0.002170 \\
\hline 0.1211 & 0.0686 & 0.1210 & 0.0685 & 0.000803 \\
\hline 0.5846 & 0.0967 & 0.5836 & 0.0965 & 0.001634 \\
\hline 0.0600 & 0.1353 & 0.0599 & 0.1352 & 0.000872 \\
\hline 0.2694 & 0.0785 & 0.2691 & 0.0784 & 0.001085 \\
\hline 0.0662 & 0.2971 & 0.0661 & 0.2967 & 0.001334 \\
\hline 0.0700 & 0.3943 & 0.0699 & 0.3936 & 0.001704 \\
\hline
\end{tabular}

${ }^{\mathrm{a}} x_{2}{ }^{(\mathrm{s})}$ : initial mole fraction of component 2 in the ternary solvent mixture, $x_{4}{ }^{(\mathrm{s})}$ : initial mole fraction of component 4 in the ternary solvent mixture; $x_{1}$ : mole fraction solubility of the solute; $x_{2}$ : mole fraction of component 2 in the quaternary solution; $x_{4}$ : mole fraction of component 4 in the quaternary solution.

\section{Auxiliary Information}

\section{Method/Apparatus/Procedure:}

Constant-temperature bath, calorimetric thermometer, and an ultraviolet/ visible spectrophotometer.

Ternary solvent mixtures were prepared by mass. Excess solute and solvent were placed in amber glass bottles and allowed to equilibrate for several days at constant temperature. Attainment of equilibrium was verified by several repetitive measurements and by approaching equilibrium from supersaturation. Aliquots of saturated solutions were transferred through a coarse filter into volumetric flasks, weighed and diluted with methanol. Molar concentrations were determined by spectrophotometric measurements at $356 \mathrm{~nm}$.

\section{Source and Purity of Chemicals:}

(1) $99+\%$, Acros Chemical Company, USA, recrystallized three times from acetone.

(2) $99 \%$, HPLC grade, Aldrich Chemical Company, Milwaukee, WI, USA.

(3) $99+\%$, anhydrous, Aldrich Chemical Company.

(4) $99+\%$, Acros Chemical Company.

Components $2-4$ were stored over molecular sieves and distilled shortly before use.

\section{Estimated Error:}

Temperature: $\pm 0.1 \mathrm{~K}$.

$x_{2}{ }^{(\mathrm{s})}: \pm 0.0001$.

$x_{4}{ }^{(\mathrm{s})}: \pm 0.0001$.

$x_{1}: \pm 1.5 \%$ (relative error).

\begin{tabular}{|c|c|}
\hline $\begin{array}{l}\text { Components: } \\
\text { (1) Anthracene; } \mathrm{C}_{14} \mathrm{H}_{10} ;[120-12-7] \\
\text { (2) Cyclohexane; } \mathrm{C}_{6} \mathrm{H}_{12} ;[110-82-7] \\
\text { (3) 1-Propanol; } \mathrm{C}_{3} \mathrm{H}_{8} \mathrm{O} ;[71-23-8] \\
\text { (4) 2-Butoxyethanol; } \mathrm{C}_{6} \mathrm{H}_{14} \mathrm{O}_{2} ; \\
\text { [111-76-2] }\end{array}$ & $\begin{array}{l}\text { Original Measurements: } \\
{ }^{25} \text { T. Deng, S. Horiuchi, L. E. Roy, } \\
\text { and W. E. Acree, Jr., J. Chem. } \\
\text { Eng. Data 44, } 258 \text { (1999). }\end{array}$ \\
\hline $\begin{array}{l}\text { Variables: } \\
T / \mathrm{K}=298.15 \text {; Solvent Composition }\end{array}$ & $\begin{array}{l}\text { Prepared by: } \\
\text { W. E. Acree, Jr. }\end{array}$ \\
\hline
\end{tabular}

Experimental Values

Solubility of anthracene in cyclohexane +1 -propanol +2 -butoxyethanol mixtures $^{\mathrm{a}}$

\begin{tabular}{lcccc}
\hline \hline$x_{2}{ }^{(\mathrm{s})}$ & $x_{4}{ }^{(\mathrm{s})}$ & $x_{2}$ & $x_{4}$ & $x_{1}$ \\
\hline 0.0000 & 0.0000 & 0.0000 & 0.0000 & 0.000591 \\
1.0000 & 0.0000 & 0.9984 & 0.0000 & 0.001553 \\
0.0000 & 1.0000 & 0.0000 & 0.9962 & 0.003785 \\
0.3071 & 0.2553 & 0.3065 & 0.2548 & 0.001884 \\
0.6708 & 0.1209 & 0.6695 & 0.1207 & 0.001931 \\
0.2124 & 0.1765 & 0.2121 & 0.1763 & 0.001402 \\
0.4688 & 0.1946 & 0.4679 & 0.1942 & 0.001936 \\
0.1057 & 0.5976 & 0.1054 & 0.5961 & 0.002560 \\
0.2189 & 0.6221 & 0.2182 & 0.6202 & 0.003045 \\
0.6923 & 0.1638 & 0.6908 & 0.1534 & 0.002182 \\
0.4298 & 0.0721 & 0.4292 & 0.0720 & 0.001487 \\
0.5103 & 0.3389 & 0.5090 & 0.3380 & 0.002520 \\
0.4151 & 0.4313 & 0.4139 & 0.4301 & 0.002801 \\
0.1162 & 0.0983 & 0.1161 & 0.0982 & 0.001007 \\
0.1596 & 0.6117 & 0.1592 & 0.6100 & 0.002790 \\
0.2454 & 0.4030 & 0.2448 & 0.4021 & 0.002262 \\
0.1545 & 0.0645 & 0.1544 & 0.0644 & 0.000957 \\
0.6533 & 0.0758 & 0.6521 & 0.0757 & 0.001791 \\
0.0793 & 0.1281 & 0.0792 & 0.1280 & 0.001038 \\
0.3297 & 0.0692 & 0.3293 & 0.0691 & 0.001272 \\
0.0866 & 0.2874 & 0.0865 & 0.2870 & 0.001531 \\
0.0952 & 0.3752 & 0.0950 & 0.3745 & 0.001855 \\
\hline
\end{tabular}

${ }^{\mathrm{a}} x_{2}{ }^{(\mathrm{s})}$ : initial mole fraction of component 2 in the ternary solvent mixture, $x_{4}{ }^{(\mathrm{s})}$ : initial mole fraction of component 4 in the ternary solvent mixture; $x_{1}$ : mole fraction solubility of the solute; $x_{2}$ : mole fraction of component 2 in the quaternary solution; $x_{4}$ : mole fraction of component 4 in the quaternary solution.

\section{Auxiliary Information}

\section{Method/Apparatus/Procedure:}

Constant-temperature bath, calorimetric thermometer, and an ultraviolet/ visible spectrophotometer.

Ternary solvent mixtures were prepared by mass. Excess solute and solvent were placed in amber glass bottles and allowed to equilibrate for several days at constant temperature. Attainment of equilibrium was verified by several repetitive measurements and by approaching equilibrium from supersaturation. Aliquots of saturated solutions were transferred through a coarse filter into volumetric flasks, weighed and diluted with methanol. Molar concentrations were determined by spectrophotometric measurements at $356 \mathrm{~nm}$. 
Source and Purity of Chemicals:

(1) $99+\%$, Acros Chemical Company, USA, recrystallized three times from acetone.

(2) $99.9+\%$, HPLC grade, Aldrich Chemical Company, Milwaukee, WI, USA

(3) $99+\%$, anhydrous, Aldrich Chemical Company.

(4) $99+\%$, Acros Chemical Company.

Components $2-4$ were stored over molecular sieves and distilled shortly before use.

Estimated Error:

Temperature: $\pm 0.1 \mathrm{~K}$.

$x_{2}{ }^{(\mathrm{s})}: \pm 0.0001$.

$x_{4}{ }^{(\mathrm{s})}: \pm 0.0001$.

$x_{1}: \pm 1.5 \%$ (relative error)

\begin{tabular}{ll}
\hline \hline Components: & Original Measurements: \\
(1) Anthracene; $\mathrm{C}_{14} \mathrm{H}_{10} ;[120-12-7]$ & ${ }^{25}$ T. Deng, S. Horiuchi, L. E. Roy, \\
(2) Cyclohexane; $\mathrm{C}_{6} \mathrm{H}_{12} ;[110-82-7]$ & and W. E. Acree, Jr., J. Chem. \\
(3) 2-Propanol; $\mathrm{C}_{3} \mathrm{H}_{8} \mathrm{O} ;[67-63-0]$ & Eng. Data 44, 258 (1999). \\
(4) 2-Butoxyethanol; $\mathrm{C}_{6} \mathrm{H}_{14} \mathrm{O}_{2} ;$ & \\
[111-76-2] & \\
\hline
\end{tabular}

\begin{tabular}{ll}
\hline Variables: & Prepared by: \\
$T / \mathrm{K}=298.15 ;$ Solvent Composition & W. E. Acree, Jr. \\
\hline
\end{tabular}

Experimental Values

Solubility of anthracene in cyclohexane +2 -propanol +2 -butoxyethanol mixtures $^{\mathrm{a}}$

\begin{tabular}{|c|c|c|c|c|}
\hline$x_{2}{ }^{(\mathrm{s})}$ & $x_{4}^{(s)}$ & $x_{2}$ & $x_{4}$ & $x_{1}$ \\
\hline 0.0000 & 0.0000 & 0.0000 & 0.0000 & 0.000411 \\
\hline 1.0000 & 0.0000 & 0.9984 & 0.0000 & 0.001553 \\
\hline 0.0000 & 1.0000 & 0.0000 & 0.9962 & 0.003785 \\
\hline 0.3097 & 0.2543 & 0.3091 & 0.2538 & 0.001786 \\
\hline 0.6769 & 0.1193 & 0.6756 & 0.1191 & 0.001912 \\
\hline 0.2147 & 0.1768 & 0.2144 & 0.1766 & 0.001283 \\
\hline 0.4718 & 0.1954 & 0.4709 & 0.1950 & 0.001899 \\
\hline 0.1050 & 0.6002 & 0.1047 & 0.5987 & 0.002560 \\
\hline 0.2178 & 0.6257 & 0.2151 & 0.6238 & 0.002977 \\
\hline 0.6909 & 0.1632 & 0.6894 & 0.1629 & 0.002115 \\
\hline 0.4356 & 0.0745 & 0.4350 & 0.0744 & 0.001342 \\
\hline 0.5148 & 0.3403 & 0.5135 & 0.3394 & 0.002607 \\
\hline 0.4198 & 0.4308 & 0.4187 & 0.4296 & 0.002712 \\
\hline 0.1191 & 0.0980 & 0.1190 & 0.0979 & 0.000846 \\
\hline 0.1608 & 0.6122 & 0.1604 & 0.6105 & 0.002776 \\
\hline 0.2476 & 0.4048 & 0.2471 & 0.4039 & 0.002169 \\
\hline 0.1560 & 0.0651 & 0.1559 & 0.0650 & 0.000817 \\
\hline 0.6558 & 0.0780 & 0.6547 & 0.0779 & 0.001723 \\
\hline 0.0805 & 0.1322 & 0.0804 & 0.1321 & 0.000874 \\
\hline 0.3352 & 0.0704 & 0.3348 & 0.0703 & 0.001153 \\
\hline 0.0898 & 0.2889 & 0.0897 & 0.2885 & 0.001360 \\
\hline 0.0938 & 0.3828 & 0.0936 & 0.3822 & 0.001676 \\
\hline
\end{tabular}

${ }^{\mathrm{a}} x_{2}{ }^{(\mathrm{s})}$ : initial mole fraction of component 2 in the ternary solvent mixture, $x_{4}{ }^{(\mathrm{s})}$ : initial mole fraction of component 4 in the ternary solvent mixture; $x_{1}$ : mole fraction solubility of the solute; $x_{2}$ : mole fraction of component 2 in the quaternary solution; $x_{4}$ : mole fraction of component 4 in the quaternary solution.

\section{Auxiliary Information}

Method/Apparatus/Procedure:

Constant-temperature bath, calorimetric thermometer, and an ultraviolet/ visible spectrophotometer.

Ternary solvent mixtures were prepared by mass. Excess solute and solvent were placed in amber glass bottles and allowed to equilibrate for several days at constant temperature. Attainment of equilibrium was verified by several repetitive measurements and by approaching equilibrium from supersaturation. Aliquots of saturated solutions were transferred through a coarse filter into volumetric flasks, weighed and diluted with methanol. Molar concentrations were determined by spectrophotometric measurements at $356 \mathrm{~nm}$.

Source and Purity of Chemicals:

(1) $99+\%$, Acros Chemical Company, USA, recrystallized three times from acetone.

(2) $99 \%$, HPLC grade, Aldrich Chemical Company, Milwaukee, WI, USA

(3) $99+\%$, anhydrous, Aldrich Chemical Company.

(4) $99+\%$, Acros Chemical Company.

Components $2-4$ were stored over molecular sieves and distilled shortly before use.

Estimated Error:

Temperature: $\pm 0.1 \mathrm{~K}$.

$x_{2}{ }^{(\mathrm{s})}: \pm 0.0001$.

$x_{4}{ }^{(\mathrm{s})}: \pm 0.0001$.

$x_{1}: \pm 1.5 \%$ (relative error).

\section{Solubility of Anthracene in Ternary Alcohol + Alcohol + Alkoxyalcohol Solvent Mixtures}

\subsection{Critical evaluation of experimental solubility data}

Deng and Acree ${ }^{22}$ determined the solubility of anthracene in four ternary propanol + butanol + 2-butoxyethanol solvent mixtures at $298.15 \mathrm{~K}$. There has only been a single experimental determination for the solubility of anthracene in each of the ternary solvent systems studied. As before, the experimental values were evaluated by curve-fitting the measured solubility data in the contributing sub-binary solvent mixtures to Eq. (1), and then using the ternary solvent version of the basic model to estimate the mole fraction solubility of anthracene dissolved in ternary alcohol + alcohol + alkoxyalcohol solvent mixtures.

The calculated $S_{23, i}$ parameters for all of the contributing binary alcohol + alcohol and alcohol + alkoxyalcohol solvent systems studied are summarized in Table 1, along with the average absolute deviation between the experimental and back-calculated mole fraction solubilities. The predictive ability of Eq. (2) is given in Table 8 for the four ternary alcohol + alcohol + alkoxyalcohol solvent systems. Examination of the numerical entries in the last column of Table 8 
TABLE 8. Summarized comparison between experimental solubilities of anthracene in ternary alcohol + alcohol + alkoxyalcohol solvent mixtures and predicted values based on Eq. (2)

\begin{tabular}{lc}
\hline \hline Ternary solvent mixture & $\%$ Dev. \\
\hline 1-Propanol + 1-butanol + 2-butoxyethanol & 1.20 \\
1-Propanol + 2-butanol + 2-butoxyethanol & 1.69 \\
2-Propanol + 1-butanol + 2-butoxyethanol & 1.42 \\
2-Propanol + 2-butanol + 2-butoxyethanol & 1.75 \\
\hline a
\end{tabular}

${ }^{\mathrm{a}} \operatorname{Dev}(\%)=(100 / N) \Sigma\left|\left[x_{1}{ }^{\mathrm{exp}}-x_{1}{ }^{\mathrm{cal}}\right] / x_{1}{ }^{\mathrm{exp}}\right|$.

indicates that Eq. (2) does provide a very accurate mathematical representation for how the measured mole fraction solubilities vary with binary solvent composition. Average percent deviations range from $1.2 \%$ to $1.8 \%$, suggesting that there are no obvious outliers in an individual ternary solvent data set. None of the experimental data points was flagged as an outlier.

The experimental anthracene solubility data for ternary propanol + butanol + 2-butoxyethanol solvent mixtures are given in Sec. 7.2.

\subsection{Anthracene solubility data in ternary alcohol + alcohol + 2-butoxyethanol solvent mixtures}

\begin{tabular}{|c|c|}
\hline $\begin{array}{l}\text { Components: } \\
\text { (1) Anthracene; } \mathrm{C}_{14} \mathrm{H}_{10} ;[120-12-7] \\
\text { (2) 1-Propanol; } \mathrm{C}_{3} \mathrm{H}_{8} \mathrm{O} ;[71-23-8] \\
\text { (3) 1-Butanol; } \mathrm{C}_{4} \mathrm{H}_{10} \mathrm{O} ;[71-36-3] \\
\text { (4) 2-Butoxyethanol; } \mathrm{C}_{6} \mathrm{H}_{14} \mathrm{O}_{2} ; \\
\text { [111-76-2] }\end{array}$ & $\begin{array}{l}\text { Original Measurements: } \\
{ }^{22} \text { T. Deng and W. E. Acree, Jr., J. } \\
\text { Chem. Eng. Data 44, } 544 \text { (1999). }\end{array}$ \\
\hline $\begin{array}{l}\text { Variables: } \\
T / \mathrm{K}=298.15 ; \text { Solvent Composition }\end{array}$ & $\begin{array}{l}\text { Prepared by: } \\
\text { W. E. Acree, Jr. }\end{array}$ \\
\hline
\end{tabular}

Experimental Values

Solubility of anthracene in 1-propanol + 1-butanol + 2-butoxyethanol mixtures $^{\mathrm{a}}$

\begin{tabular}{lcccc}
\hline \hline$x_{2}{ }^{(\mathrm{s})}$ & $x_{4}{ }^{(\mathrm{s})}$ & $x_{2}$ & $x_{4}$ & $x_{1}$ \\
\hline 0.0000 & 0.0000 & 0.0000 & 0.0000 & 0.000591 \\
1.0000 & 0.0000 & 0.9992 & 0.0000 & 0.000801 \\
0.0000 & 1.0000 & 0.0000 & 0.9962 & 0.003785 \\
0.4227 & 0.2408 & 0.4222 & 0.2405 & 0.001293 \\
0.7688 & 0.0966 & 0.7681 & 0.0965 & 0.000853 \\
0.3140 & 0.1805 & 0.3136 & 0.1803 & 0.001141 \\
0.5866 & 0.1713 & 0.5860 & 0.1711 & 0.001061 \\
0.1473 & 0.6026 & 0.1470 & 0.6012 & 0.002376 \\
0.2886 & 0.5871 & 0.2879 & 0.5857 & 0.002305 \\
0.7796 & 0.1294 & 0.7789 & 0.1293 & 0.000918 \\
0.5681 & 0.0647 & 0.5676 & 0.0646 & 0.000853 \\
0.6189 & 0.2802 & 0.6180 & 0.2798 & 0.001387 \\
0.5212 & 0.3734 & 0.5203 & 0.3728 & 0.001651 \\
0.1887 & 0.1077 & 0.1885 & 0.1076 & 0.001003
\end{tabular}

$\begin{array}{lllll}0.2240 & 0.5947 & 0.2235 & 0.5933 & 0.002333 \\ 0.3407 & 0.3817 & 0.3401 & 0.3811 & 0.001696 \\ 0.2390 & 0.0719 & 0.2388 & 0.0718 & 0.000915 \\ 0.7572 & 0.0641 & 0.7566 & 0.0641 & 0.000778 \\ 0.1274 & 0.1453 & 0.1273 & 0.1451 & 0.001109 \\ 0.4630 & 0.0677 & 0.4626 & 0.0676 & 0.000887 \\ 0.1403 & 0.3062 & 0.1401 & 0.3057 & 0.001544 \\ 0.1413 & 0.4004 & 0.1410 & 0.3997 & 0.001805\end{array}$

${ }^{\mathrm{a}} x_{2}{ }^{(\mathrm{s})}$ : initial mole fraction of component 2 in the ternary solvent mixture, $x_{4}{ }^{(\mathrm{s})}$ : initial mole fraction of component 4 in the ternary solvent mixture; $x_{1}$ : mole fraction solubility of the solute; $x_{2}$ : mole fraction of component 2 in the quaternary solution; $x_{4}$ : mole fraction of component 4 in the quaternary solution.

\section{Auxiliary Information}

\section{Method/Apparatus/Procedure:}

Constant-temperature bath, calorimetric thermometer, and an ultraviolet/ visible spectrophotometer.

Ternary solvent mixtures were prepared by mass. Excess solute and solvent were placed in amber glass bottles and allowed to equilibrate for several days at constant temperature. Attainment of equilibrium was verified by several repetitive measurements and by approaching equilibrium from supersaturation. Aliquots of saturated solutions were transferred through a coarse filter into volumetric flasks, weighed and diluted with methanol. Molar concentrations were determined by spectrophotometric measurements at $356 \mathrm{~nm}$.

Source and Purity of Chemicals:

(1) $99+\%$, Acros Chemical Company, USA, recrystallized three times from acetone.

(2) $99+\%$, anhydrous, Aldrich Chemical Company, Milwaukee, WI, USA.

(3) $99.8+\%$, HPLC grade, Aldrich Chemical Company.

(4) $99+\%$, Acros Chemical Company.

Components 2-4 were stored over molecular sieves and distilled shortly before use.

Estimated Error:

Temperature: $\pm 0.1 \mathrm{~K}$.

$x_{2}{ }^{(\mathrm{s})}: \pm 0.0001$.

$x_{4}{ }^{(\mathrm{s})}: \pm 0.0001$.

$x_{1}: \pm 1.5 \%$ (relative error).

\begin{tabular}{|c|c|}
\hline $\begin{array}{l}\text { Components: } \\
\text { (1) Anthracene; } \mathrm{C}_{14} \mathrm{H}_{10} ;[120-12-7] \\
\text { (2) 1-Propanol; } \mathrm{C}_{3} \mathrm{H}_{8} \mathrm{O} ;[71-23-8] \\
\text { (3) 2-Butanol; } \mathrm{C}_{4} \mathrm{H}_{10} \mathrm{O} ;[78-92-2] \\
\text { (4) 2-Butoxyethanol; } \mathrm{C}_{6} \mathrm{H}_{14} \mathrm{O}_{2} ; \\
\text { [111-76-2] }\end{array}$ & $\begin{array}{l}\text { Original Measurements: } \\
{ }^{22} \text { T. Deng and W. E. Acree, Jr., J. } \\
\text { Chem. Eng. Data 44, } 544 \text { (1999). }\end{array}$ \\
\hline $\begin{array}{l}\text { Variables: } \\
T / \mathrm{K}=298.15 ; \text { Solvent Composition }\end{array}$ & $\begin{array}{l}\text { Prepared by: } \\
\text { W. E. Acree, Jr. }\end{array}$ \\
\hline
\end{tabular}




\section{Experimental Values}

Solubility of anthracene in 1-propanol +2 -butanol +2 -butoxyethanol mixtures $^{\mathrm{a}}$

\begin{tabular}{lcccc}
\hline \hline$x_{2}{ }^{(\mathrm{s})}$ & $x_{4}{ }^{(\mathrm{s})}$ & $x_{2}$ & $x_{4}$ & $x_{1}$ \\
\hline 0.0000 & 0.0000 & 0.0000 & 0.0000 & 0.000585 \\
1.0000 & 0.0000 & 0.9994 & 0.0000 & 0.000591 \\
0.0000 & 1.0000 & 0.0000 & 0.9962 & 0.003785 \\
0.4230 & 0.2394 & 0.4225 & 0.2391 & 0.001192 \\
0.7728 & 0.0950 & 0.7722 & 0.0949 & 0.000820 \\
0.3143 & 0.1790 & 0.3140 & 0.1788 & 0.001027 \\
0.5914 & 0.1698 & 0.5908 & 0.1696 & 0.001018 \\
0.1541 & 0.6047 & 0.1537 & 0.6033 & 0.002330 \\
0.2959 & 0.5833 & 0.2952 & 0.5820 & 0.002304 \\
0.7818 & 0.1282 & 0.7811 & 0.1281 & 0.000905 \\
0.5665 & 0.0657 & 0.5661 & 0.0656 & 0.000738 \\
0.6186 & 0.2816 & 0.6178 & 0.2812 & 0.001326 \\
0.5234 & 0.3723 & 0.5226 & 0.3717 & 0.001602 \\
0.1889 & 0.1058 & 0.1887 & 0.1057 & 0.000840 \\
0.2268 & 0.5924 & 0.2263 & 0.5910 & 0.002283 \\
0.3386 & 0.3858 & 0.3381 & 0.3852 & 0.001612 \\
0.2415 & 0.0697 & 0.2413 & 0.0696 & 0.000751 \\
0.7612 & 0.0638 & 0.7606 & 0.0638 & 0.000745 \\
0.1300 & 0.1492 & 0.1299 & 0.1491 & 0.000960 \\
0.4614 & 0.0682 & 0.4611 & 0.0681 & 0.000741 \\
0.1375 & 0.3104 & 0.1373 & 0.3100 & 0.001401 \\
0.1411 & 0.4016 & 0.1409 & 0.4009 & 0.001684 \\
\hline $\mathrm{a}_{x_{2}}{ }^{(\mathrm{s})}$. initial mole fraction of component 2 in the ternary solvent mixture, $x_{4}^{(\mathrm{s})}$
\end{tabular}

. initial mole fraction of component 4 in the ternary solvent mixture; $x_{1}$ : mole fraction solubility of the solute; $x_{2}$ : mole fraction of component 2 in the quaternary solution; $x_{4}$ : mole fraction of component 4 in the quaternary solution.

\section{Auxiliary Information}

\section{Method/Apparatus/Procedure:}

Constant-temperature bath, calorimetric thermometer, and an ultraviolet/ visible spectrophotometer.

Ternary solvent mixtures were prepared by mass. Excess solute and solvent were placed in amber glass bottles and allowed to equilibrate for several days at constant temperature. Attainment of equilibrium was verified by several repetitive measurements and by approaching equilibrium from supersaturation. Aliquots of saturated solutions were transferred through a coarse filter into volumetric flasks, weighed and diluted with methanol. Molar concentrations were determined by spectrophotometric measurements at $356 \mathrm{~nm}$.

\section{Source and Purity of Chemicals:}

(1) $99+\%$, Acros Chemical Company, USA, recrystallized three times from acetone.

(2) $99+\%$, anhydrous, Aldrich Chemical Company, Milwaukee, WI, USA.

(3) $99+\%$, anhydrous, Aldrich Chemical Company.

(4) $99+\%$, Acros Chemical Company.

Components 2-4 were stored over molecular sieves and distilled shortly before use.

\section{Estimated Error:}

Temperature: $\pm 0.1 \mathrm{~K}$

$x_{2}^{(\mathrm{s})}: \pm 0.0001$.

$x_{4}{ }^{(\mathrm{s})}: \pm 0.0001$

$x_{1}: \pm 1.5 \%$ (relative error)

\begin{tabular}{|c|c|}
\hline $\begin{array}{l}\text { Components: } \\
\text { (1) Anthracene; } \mathrm{C}_{14} \mathrm{H}_{10} ;[120-12-7] \\
\text { (2) 2-Propanol; } \mathrm{C}_{3} \mathrm{H}_{8} \mathrm{O} ;[67-63-0] \\
\text { (3) 1-Butanol; } \mathrm{C}_{4} \mathrm{H}_{10} \mathrm{O} ;[71-36-3] \\
\text { (4) 2-Butoxyethanol; } \mathrm{C}_{6} \mathrm{H}_{14} \mathrm{O}_{2} \text {; } \\
\text { [111-76-2] }\end{array}$ & $\begin{array}{l}\text { Original Measurements: } \\
{ }^{22} \text { T. Deng and W. E. Acree, Jr., J. } \\
\text { Chem. Eng. Data 44, } 544 \text { (1999). }\end{array}$ \\
\hline $\begin{array}{l}\text { Variables: } \\
T / \mathrm{K}=298.15 \text {; Solvent Composition }\end{array}$ & $\begin{array}{l}\text { Prepared by: } \\
\text { W. E. Acree, Jr. }\end{array}$ \\
\hline
\end{tabular}

Experimental Values

Solubility of anthracene in 2-propanol + 1-butanol + 2-butoxyethanol mixtures $^{\mathrm{a}}$

\begin{tabular}{lcccc}
\hline \hline$x_{2}^{(\mathrm{s})}$ & $x_{4}{ }^{(\mathrm{s})}$ & $x_{2}$ & $x_{4}$ & $x_{1}$ \\
\hline 0.0000 & 0.0000 & 0.0000 & 0.0000 & 0.000801 \\
1.0000 & 0.0000 & 0.9996 & 0.0000 & 0.000411 \\
0.0000 & 1.0000 & 0.0000 & 0.9962 & 0.003785 \\
0.4100 & 0.2420 & 0.4095 & 0.2417 & 0.001193 \\
0.7641 & 0.0968 & 0.7636 & 0.0967 & 0.000695 \\
0.3042 & 0.1807 & 0.3039 & 0.1805 & 0.001059 \\
0.5813 & 0.1716 & 0.5808 & 0.1714 & 0.000940 \\
0.1519 & 0.6011 & 0.1515 & 0.5997 & 0.002359 \\
0.2894 & 0.5887 & 0.2887 & 0.5874 & 0.002248 \\
0.7780 & 0.1301 & 0.7774 & 0.1300 & 0.000766 \\
0.5599 & 0.0657 & 0.5595 & 0.0657 & 0.000706 \\
0.6128 & 0.2848 & 0.6121 & 0.2845 & 0.001210 \\
0.5170 & 0.3765 & 0.5161 & 0.3759 & 0.001507 \\
0.1800 & 0.1079 & 0.1798 & 0.1078 & 0.000987 \\
0.2177 & 0.5985 & 0.2172 & 0.5972 & 0.002210 \\
0.3312 & 0.3888 & 0.3307 & 0.3882 & 0.001626 \\
0.2412 & 0.0714 & 0.2410 & 0.0713 & 0.000869 \\
0.7557 & 0.0644 & 0.7552 & 0.0644 & 0.000633 \\
0.1263 & 0.1474 & 0.1262 & 0.1472 & 0.001106 \\
0.4570 & 0.0677 & 0.4567 & 0.0676 & 0.000753 \\
0.1349 & 0.3110 & 0.1347 & 0.3106 & 0.001523 \\
0.1378 & 0.4032 & 0.1376 & 0.4025 & 0.001765 \\
\hline
\end{tabular}

${ }^{\mathrm{a}} x_{2}{ }^{(\mathrm{s})}$ : initial mole fraction of component 2 in the ternary solvent mixture, $x_{4}{ }^{(\mathrm{s})}$ : initial mole fraction of component 4 in the ternary solvent mixture; $x_{1}$ : mole fraction solubility of the solute; $x_{2}$ : mole fraction of component 2 in the quaternary solution; $x_{4}$ : mole fraction of component 4 in the quaternary solution.

\section{Auxiliary Information}

\section{Method/Apparatus/Procedure:}

Constant-temperature bath, calorimetric thermometer, and an ultraviolet/ visible spectrophotometer.

Ternary solvent mixtures were prepared by mass. Excess solute and solvent were placed in amber glass bottles and allowed to equilibrate for several days at constant temperature. Attainment of equilibrium was verified by several repetitive measurements and by approaching equilibrium from supersaturation. Aliquots of saturated solutions were transferred through a coarse filter into volumetric flasks, weighed and diluted with methanol. Molar concentrations were determined by spectrophotometric measurements at $356 \mathrm{~nm}$.

Source and Purity of Chemicals:

(1) $99+\%$, Acros Chemical Company, USA, recrystallized three times from acetone.

(2) $99+\%$, anhydrous, Aldrich Chemical Company, Milwaukee, WI, USA.

(3) $99.8+\%$, HPLC grade, Aldrich Chemical Company.

(4) $99+\%$, Acros Chemical Company.

Components 2-4 were stored over molecular sieves and distilled shortly before use. 
Estimated Error:

Temperature: $\pm 0.1 \mathrm{~K}$.

$x_{2}{ }^{(\mathrm{s})}: \pm 0.0001$.

$x_{4}{ }^{(\mathrm{s})}: \pm 0.0001$.

$x_{1}: \pm 1.5 \%$ (relative error).

\begin{tabular}{ll}
\hline \hline Components: & $\begin{array}{l}\text { Original Measurements: } \\
\text { (1) Anthracene; } \mathrm{C}_{14} \mathrm{H}_{10} ;[120-12-7]\end{array}$ \\
$\begin{array}{ll}{ }^{22} \mathrm{~T} \text {. Deng and W. E. Acree, Jr., J. } \\
\text { (2) 2-Propanol; } \mathrm{C}_{3} \mathrm{H}_{8} \mathrm{O} ;[67-63-0]\end{array}$ & Chem. Eng. Data 44, 544 (1999). \\
(3) 2-Butanol; $\mathrm{C}_{4} \mathrm{H}_{10} \mathrm{O} ;[78-92-2]$ & \\
(4) 2-Butoxyethanol; $\mathrm{C}_{6} \mathrm{H}_{14} \mathrm{O}_{2} ;$ & \\
{$[111-76-2]$} & Prepared by: \\
\hline Variables: & W. E. Acree, Jr. \\
$T / \mathrm{K}=298.15 ;$ Solvent Composition &
\end{tabular}

Experimental Values

Solubility of anthracene in 2-propanol +2 -butanol +2 -butoxyethanol mixtures $^{\mathrm{a}}$

\begin{tabular}{lcccc}
\hline \hline$x_{2}{ }^{(\mathrm{s})}$ & $x_{4}{ }^{(\mathrm{s})}$ & $x_{2}$ & $x_{4}$ & $x_{1}$ \\
\hline 0.0000 & 0.0000 & 0.0000 & 0.0000 & 0.000585 \\
1.0000 & 0.0000 & 0.9996 & 0.0000 & 0.000411 \\
0.0000 & 1.0000 & 0.0000 & 0.9962 & 0.003785 \\
0.4116 & 0.2397 & 0.4112 & 0.2394 & 0.001088 \\
0.7613 & 0.0985 & 0.7608 & 0.0984 & 0.000664 \\
0.3082 & 0.1799 & 0.3079 & 0.1797 & 0.000938 \\
0.5850 & 0.1717 & 0.5845 & 0.1716 & 0.000873 \\
0.1487 & 0.6039 & 0.1484 & 0.6025 & 0.002280 \\
0.2887 & 0.5870 & 0.2881 & 0.5857 & 0.002202 \\
0.7772 & 0.1299 & 0.7766 & 0.1298 & 0.000750 \\
0.5594 & 0.0659 & 0.5590 & 0.0659 & 0.000628 \\
0.6114 & 0.2853 & 0.6107 & 0.2850 & 0.001189 \\
0.5166 & 0.3768 & 0.5159 & 0.3763 & 0.001448 \\
0.1813 & 0.1081 & 0.1812 & 0.1080 & 0.000802 \\
0.2181 & 0.5944 & 0.2176 & 0.5931 & 0.002215 \\
0.3340 & 0.3857 & 0.3335 & 0.3851 & 0.001507 \\
0.2362 & 0.0701 & 0.2390 & 0.0701 & 0.000712 \\
0.7591 & 0.0605 & 0.7587 & 0.0605 & 0.000590 \\
0.1265 & 0.1483 & 0.1264 & 0.1482 & 0.000917 \\
0.4599 & 0.0674 & 0.4596 & 0.0674 & 0.000651 \\
0.1341 & 0.3120 & 0.1339 & 0.3117 & 0.001360 \\
0.1440 & 0.4016 & 0.1438 & 0.4009 & 0.001627 \\
\hline$x_{2}(5)$ & &
\end{tabular}

${ }^{\mathrm{a}} x_{2}{ }^{(\mathrm{s})}$ : initial mole fraction of component 2 in the ternary solvent mixture, $x_{4}{ }^{(\mathrm{s})}$ : initial mole fraction of component 4 in the ternary solvent mixture; $x_{1}$ : mole fraction solubility of the solute; $x_{2}$ : mole fraction of component 2 in the quaternary solution; $x_{4}$ : mole fraction of component 4 in the quaternary solution.

\section{Auxiliary Information}

\section{Method/Apparatus/Procedure:}

Constant-temperature bath, calorimetric thermometer, and an ultraviolet/ visible spectrophotometer.

Ternary solvent mixtures were prepared by mass. Excess solute and solvent were placed in amber glass bottles and allowed to equilibrate for several days at constant temperature. Attainment of equilibrium was verified by several repetitive measurements and by approaching equilibrium from supersaturation. Aliquots of saturated solutions were transferred through a coarse filter into volumetric flasks, weighed and diluted with methanol. Molar concentrations were determined by spectrophotometric measurements at $356 \mathrm{~nm}$.
Source and Purity of Chemicals:

(1) $99+\%$, Acros Chemical Company, USA, recrystallized three times from acetone.

(2) $99+\%$, anhydrous, Aldrich Chemical Company, Milwaukee, WI, USA.

(3) $99+\%$, anhydrous, Aldrich Chemical Company.

(4) $99+\%$, Acros Chemical Company.

Components $2-4$ were stored over molecular sieves and distilled shortly before use.

Estimated Error:

Temperature: $\pm 0.1 \mathrm{~K}$.

$x_{2}{ }^{(s)}: \pm 0.0001$.

$x_{4}{ }^{(\mathrm{s})}: \pm 0.0001$.

$x_{1}: \pm 1.5 \%$ (relative error).

\section{Solubility of Anthracene in Ternary Alkane + Alkanone + Alcohol Solvent Mixtures}

\subsection{Critical evaluation of experimental solubility data}

Shayanfar et al. ${ }^{10}$ determined the solubility of anthracene in ternary solvent mixtures containing 2,2,4-trimethylpentane and propanone with methanol, ethanol, 1-propanol and 2propanol at $298.15 \mathrm{~K}$. The experimental solubility data are given in Sec. 8.2. There has only been a single experimental determination for the solubility of anthracene in each of the ternary solvent systems studied. The experimental values were evaluated by curve-fitting the measured solubility data to the molarity-based version of the Combined NIBS/Redlich-Kister equation

$$
\begin{aligned}
\ln c_{1}{ }^{\text {sat }}= & x_{2}{ }^{(\mathrm{s})} \ln \left(c_{1}{ }^{\mathrm{sat}}\right)_{2}+x_{3}{ }^{(\mathrm{s})} \ln \left(c_{1}{ }^{\mathrm{sat}}\right)_{3} \\
& +x_{2}{ }^{(\mathrm{s})} x_{3}{ }^{(\mathrm{s})} \sum_{j=0}^{r} S_{23, j}\left(x_{2}{ }^{(\mathrm{s})}-x_{3}{ }^{(\mathrm{s})}\right)^{j}
\end{aligned}
$$

and then using the ternary solvent version of the basic model to estimate the molar solubility of anthracene dissolved in ternary alkane + alkanone + alcohol solvent mixtures. The predictive expression takes the form of

$$
\begin{aligned}
\ln c_{1}{ }^{\mathrm{sat}}= & x_{2}{ }^{(\mathrm{s})} \ln \left(c_{1}{ }^{\mathrm{sat}}\right)_{2}+x_{3}{ }^{(\mathrm{s})} \ln \left(c_{1}^{\mathrm{sat}}\right)_{3}+x_{4}{ }^{(\mathrm{s})} \ln \left(c_{1}{ }^{\mathrm{sat}}\right)_{4} \\
& +x_{2}{ }^{(\mathrm{s})} x_{3}{ }^{(\mathrm{s})} \sum_{j=0}^{r} S_{23, j}\left(x_{2}{ }^{(\mathrm{s})}-x_{3}{ }^{(\mathrm{s})}\right)^{j} \\
& +x_{2}{ }^{(\mathrm{s})} x_{4}{ }^{(\mathrm{s})} \sum_{k=0}^{s} S_{24, k}\left(x_{2}{ }^{(\mathrm{s})}-x_{4}{ }^{(\mathrm{s})}\right)^{k} \\
& +x_{3}{ }^{(\mathrm{s})} x_{4}{ }^{(\mathrm{s})} \sum_{l=0}^{t} S_{34, l}\left(x_{3}{ }^{(\mathrm{s})}-x_{4}{ }^{(\mathrm{s})}\right)^{l} .
\end{aligned}
$$

In the above equation, $x_{i}{ }^{(\mathrm{s})}$ refer to the initial mole fraction solvent composition of component $i$ calculated as if the solute were not present, and $\left(c_{1}{ }^{\text {sat }}\right)_{i}$ denotes the measured molar solute solubility in pure solvent $i$. The various $S_{23, i}$ parameters are determined by regression analysis. The authors stated that the average mean percent deviation between experimental and 
predicted values based on Eq. (5) for anthracene dissolved in ternary 2,2,4-trimethylpentane + propanone + methanol, 2,2,4-trimethylpentane + propanone + ethanol, 2,2,4-trimethylpentane + propanone +1 -propanol, and 2,2,4-trimethylpentane + propanone +2 -propanol mixtures were $15.7 \%, 9.8 \%, 16.1 \%$, and $12.3 \%$, respectively.

\subsection{Anthracene solubility data in ternary $2,2,4-$ trimethylpentane + propanone + alcohol solvent mixtures}

\begin{tabular}{|c|c|}
\hline $\begin{array}{l}\text { Components: } \\
\text { (1) Anthracene; } \mathrm{C}_{14} \mathrm{H}_{10} ;[120-12-7] \\
\text { (2) } 2,2,4-\text { Trimethylpentane; } \mathrm{C}_{8} \mathrm{H}_{18} \text {; } \\
\text { [540-84-1] } \\
\text { (3) Propanone; } \mathrm{C}_{3} \mathrm{H}_{6} \mathrm{O} ;[67-64-1] \\
\text { (4) Methanol; } \mathrm{CH}_{4} \mathrm{O} ;[67-56-1]\end{array}$ & $\begin{array}{l}\text { Original Measurements: } \\
{ }^{10} \text { A. Shayanfar, S. Soltani, F. } \\
\text { Jabbaribar, E. Tamizi, W. E. } \\
\text { Acree, Jr., and A. Jouyban, J. } \\
\text { Chem. Eng. Data 53, } 890 \text { (2008). }\end{array}$ \\
\hline $\begin{array}{l}\text { Variables: } \\
T / \mathrm{K}=298.15 \text {; Solvent Composition }\end{array}$ & $\begin{array}{l}\text { Prepared by: } \\
\text { W. E. Acree, Jr. }\end{array}$ \\
\hline
\end{tabular}

Experimental Values

Solubility of anthracene in 2,2,4-trimethylmethylpentane + propanone + methanol mixtures ${ }^{\mathrm{a}}$

\begin{tabular}{|c|c|c|}
\hline$x_{2}{ }^{(\mathrm{s})}$ & $x_{3}{ }^{(\mathrm{s})}$ & $c_{1}$ \\
\hline 0.000 & 0.000 & 0.00580 \\
\hline 1.000 & 0.000 & 0.00669 \\
\hline 0.000 & 1.000 & 0.04353 \\
\hline 0.402 & 0.129 & 0.014 \\
\hline 0.449 & 0.289 & 0.019 \\
\hline 0.380 & 0.263 & 0.018 \\
\hline 0.212 & 0.095 & 0.014 \\
\hline 0.251 & 0.339 & 0.022 \\
\hline 0.277 & 0.498 & 0.028 \\
\hline 0.150 & 0.084 & 0.013 \\
\hline 0.207 & 0.582 & 0.029 \\
\hline 0.124 & 0.371 & 0.024 \\
\hline 0.104 & 0.470 & 0.027 \\
\hline 0.061 & 0.069 & 0.013 \\
\hline 0.069 & 0.232 & 0.016 \\
\hline 0.092 & 0.722 & 0.033 \\
\hline 0.045 & 0.101 & 0.011 \\
\hline 0.064 & 0.674 & 0.033 \\
\hline 0.033 & 0.296 & 0.019 \\
\hline 0.035 & 0.394 & 0.022 \\
\hline 0.030 & 0.170 & 0.013 \\
\hline 0.040 & 0.632 & 0.028 \\
\hline
\end{tabular}

${ }^{\mathrm{a}} x_{2}{ }^{(\mathrm{s})}$ : initial mole fraction of component 2 in the ternary solvent mixture, $x_{3}{ }^{(\mathrm{s})}$ initial mole fraction of component 3 in the ternary solvent mixture; $c_{1}$ : molar solubility of the solute.

\section{Auxiliary Information}

\section{Method/Apparatus/Procedure:}

Shaker bath, incubator equipped with temperature controlling system, and an ultraviolet/visible spectrophotometer.
Ternary solvent mixtures were prepared by volume. Excess solute and solvent were placed in sealed containers and allowed to equilibrate for three days at constant temperature. Attainment of equilibrium was verified by several repetitive measurements and by approaching equilibrium from supersaturation. Aliquots of saturated solutions were transferred through a hydrophobic Drapore filters $(0.45 \mu \mathrm{m}$, Millipore, Ireland) into tared volumetric flasks, weighed and diluted with methanol. Molar concentrations were determined by spectrophotometric measurements at $356 \mathrm{~nm}$. The measured molar solubilities were converted to mole fraction solubilities using the measured densities of the saturated solutions.

\section{Source and Purity of Chemicals:}

(1) $96 \%$, Fluka Chemical Company, recrystallized several times from acetone and ethyl ethanoate.

(2) $99+\%$, Merck Chemical Company, Germany.

(3) Purity and chemical source not specified.

(4) $99.5 \%$, Merck Chemical Company, Germany.

\section{Estimated Error:}

Temperature: $\pm 0.2 \mathrm{~K}$.

$x_{2}{ }^{(s)}: \pm 0.005$.

$x_{3}{ }^{(s)}: \pm 0.005$.

$c_{1}: \pm 2.0 \%$ (relative error).

\begin{tabular}{|c|c|}
\hline $\begin{array}{l}\text { Components: } \\
\text { (1) Anthracene; } \mathrm{C}_{14} \mathrm{H}_{10} ;[120-12-7] \\
\text { (2) } 2,2,4-\text {-Trimethylpentane; } \mathrm{C}_{8} \mathrm{H}_{18} \text {; } \\
\text { [540-84-1] } \\
\text { (3) Propanone; } \mathrm{C}_{3} \mathrm{H}_{6} \mathrm{O} ;[67-64-1] \\
\text { (4) Ethanol; } \mathrm{C}_{2} \mathrm{H}_{6} \mathrm{O} ;[64-17-5]\end{array}$ & $\begin{array}{l}\text { Original Measurements: } \\
{ }^{10} \text { A. Shayanfar, S. Soltani, F. } \\
\text { Jabbaribar, E. Tamizi, W. E. } \\
\text { Acree, Jr., and A. Jouyban, J. } \\
\text { Chem. Eng. Data 53, } 890 \text { (2008). }\end{array}$ \\
\hline $\begin{array}{l}\text { Variables: } \\
T / \mathrm{K}=298.15 \text {; Solvent Composition }\end{array}$ & $\begin{array}{l}\text { Prepared by: } \\
\text { W. E. Acree, Jr. }\end{array}$ \\
\hline
\end{tabular}

Experimental Values

Solubility of anthracene in 2,2,4-trimethylmethylpentane + propanone + ethanol mixtures ${ }^{\mathrm{a}}$

\begin{tabular}{lll}
\hline \hline$x_{2}{ }^{(\mathrm{s})}$ & $x_{3}{ }^{(\mathrm{s})}$ & $c_{1}$ \\
\hline 0.000 & 0.000 & 0.00868 \\
1.000 & 0.000 & 0.00669 \\
0.000 & 1.000 & 0.04353 \\
0.465 & 0.150 & 0.014 \\
0.486 & 0.313 & 0.019 \\
0.423 & 0.293 & 0.020 \\
0.265 & 0.119 & 0.014 \\
0.285 & 0.385 & 0.023 \\
0.296 & 0.533 & 0.030 \\
0.193 & 0.108 & 0.016 \\
0.220 & 0.620 & 0.034 \\
0.145 & 0.435 & 0.027 \\
0.119 & 0.536 & 0.031 \\
0.081 & 0.092 & 0.014 \\
0.086 & 0.290 & 0.022 \\
0.097 & 0.763 & 0.038 \\
0.060 & 0.134 & 0.016 \\
0.069 & 0.729 & 0.035 \\
0.041 & 0.367 & 0.025 \\
0.042 & 0.472 & 0.029 \\
0.039 & 0.221 & 0.020 \\
0.044 & 0.698 & 0.036 \\
\hline$x_{2}$ & $1 \mathrm{n}$ &
\end{tabular}

${ }^{\mathrm{a}} x_{2}{ }^{(\mathrm{s})}$ : initial mole fraction of component 2 in the ternary solvent mixture, $x_{3}{ }^{(\mathrm{s})}$ : initial mole fraction of component 3 in the ternary solvent mixture; $c_{1}$ : molar solubility of the solute. 


\section{Auxiliary Information}

\section{Method/Apparatus/Procedure:}

Shaker bath, incubator equipped with temperature controlling system, and an ultraviolet/visible spectrophotometer.

Ternary solvent mixtures were prepared by volume. Excess solute and solvent were placed in sealed containers and allowed to equilibrate for three days at constant temperature. Attainment of equilibrium was verified by several repetitive measurements and by approaching equilibrium from supersaturation. Aliquots of saturated solutions were transferred through a hydrophobic Drapore filters $(0.45 \mu \mathrm{m}$, Millipore, Ireland $)$ into tared volumetric flasks, weighed and diluted with methanol. Molar concentrations were determined by spectrophotometric measurements at $356 \mathrm{~nm}$. The measured molar solubilities were converted to mole fraction solubilities using the measured densities of the saturated solutions.

\section{Source and Purity of Chemicals:}

(1) $96 \%$, Fluka Chemical Company, recrystallized several times from acetone and ethyl ethanoate.

(2) $99+\%$, Merck Chemical Company, Germany.

(3) Purity and chemical source not specified.

(4) $99.9 \%$, absolute, Merck Chemical Company, Germany.

\section{Estimated Error:}

Temperature: $\pm 0.2 \mathrm{~K}$.

$x_{2}{ }^{(\mathrm{s})}: \pm 0.005$.

$x_{3}{ }^{(\mathrm{s})}: \pm 0.005$.

$c_{1}: \pm 2.0 \%$ (relative error).

\section{Components:}

(1) Anthracene; $\mathrm{C}_{14} \mathrm{H}_{10} ;[120-12-7]$

(2) 2,2,4-Trimethylpentane; $\mathrm{C}_{8} \mathrm{H}_{18}$;

[540-84-1]

(3) Propanone; $\mathrm{C}_{3} \mathrm{H}_{6} \mathrm{O}$; [67-64-1]

(4) 1-Propanol; $\mathrm{C}_{3} \mathrm{H}_{8} \mathrm{O}$; [71-23-8]

\begin{tabular}{ll}
\hline Variables: & Prepared by: \\
$T / \mathrm{K}=298.15 ;$ Solvent Composition & W. E. Acree, Jr. \\
\hline
\end{tabular}

\section{Experimental Values}

Solubility of anthracene in 2,2,4-trimethylmethylpentane + propanone +1 propanol mixtures ${ }^{\mathrm{a}}$

\begin{tabular}{lll}
\hline \hline$x_{2}{ }^{(\mathrm{s})}$ & $x_{3}{ }^{(\mathrm{s})}$ & \multicolumn{1}{c}{$c_{1}$} \\
\hline 0.000 & 0.000 & 0.00822 \\
1.000 & 0.000 & 0.00669 \\
0.000 & 1.000 & 0.04353 \\
0.513 & 0.165 & 0.016 \\
0.511 & 0.328 & 0.019 \\
0.454 & 0.315 & 0.021 \\
0.311 & 0.140 & 0.017 \\
0.309 & 0.418 & 0.026 \\
0.309 & 0.556 & 0.028 \\
0.232 & 0.130 & 0.017 \\
0.229 & 0.645 & 0.036 \\
0.161 & 0.484 & 0.031 \\
0.130 & 0.584 & 0.034 \\
0.102 & 0.114 & 0.014
\end{tabular}

$\begin{array}{lll}0.101 & 0.342 & 0.028 \\ 0.100 & 0.789 & 0.040 \\ 0.074 & 0.166 & 0.017 \\ 0.073 & 0.766 & 0.040 \\ 0.048 & 0.428 & 0.030 \\ 0.047 & 0.534 & 0.033 \\ 0.048 & 0.269 & 0.023 \\ 0.047 & 0.745 & 0.043\end{array}$

${ }^{\mathrm{a}} x_{2}{ }^{(\mathrm{s})}$ : initial mole fraction of component 2 in the ternary solvent mixture, $x_{3}{ }^{(\mathrm{s})}$ : initial mole fraction of component 3 in the ternary solvent mixture; $c_{1}$ : molar solubility of the solute.

Auxiliary Information

Method/Apparatus/Procedure:

Shaker bath, incubator equipped with temperature controlling system, and an ultraviolet/visible spectrophotometer.

Ternary solvent mixtures were prepared by volume. Excess solute and solvent were placed in sealed containers and allowed to equilibrate for three days at constant temperature. Attainment of equilibrium was verified by several repetitive measurements and by approaching equilibrium from supersaturation. Aliquots of saturated solutions were transferred through a hydrophobic Drapore filters $(0.45 \mu \mathrm{m}$, Millipore, Ireland) into tared volumetric flasks, weighed and diluted with methanol. Molar concentrations were determined by spectrophotometric measurements at $356 \mathrm{~nm}$. The measured molar solubilities were converted to mole fraction solubilities using the measured densities of the saturated solutions.

\section{Source and Purity of Chemicals:}

(1) $96 \%$, Fluka Chemical Company, recrystallized several times from acetone and ethyl ethanoate.

(2) $99+\%$, Merck Chemical Company, Germany.

(3) Purity and chemical source not specified.

(4) $99.5 \%$, Merck Chemical Company, Germany.

\section{Estimated Error:}

Temperature: $\pm 0.2 \mathrm{~K}$.

$x_{2}{ }^{(\mathrm{s})}: \pm 0.005$.

$x_{3}{ }^{(\mathrm{s})}: \pm 0.005$.

$c_{1}: \pm 2.0 \%$ (relative error).

\begin{tabular}{ll}
\hline \hline Components: & Original Measurements: \\
(1) Anthracene; $\mathrm{C}_{14} \mathrm{H}_{10} ;[120-12-7]$ & ${ }^{10}$ A. Shayanfar, S. Soltani, F. \\
(2) 2,2,4-Trimethylpentane; $\mathrm{C}_{8} \mathrm{H}_{18} ;$ & Jabbaribar, E. Tamizi, W. E. \\
[540-84-1] & Acree, Jr., and A. Jouyban, J. \\
(3) Propanone; $\mathrm{C}_{3} \mathrm{H}_{6} \mathrm{O} ;[67-64-1]$ & Chem. Eng. Data 53, 890 (2008). \\
(4) 2-Propanol; $\mathrm{C}_{3} \mathrm{H}_{8} \mathrm{O} ;[67-63-0]$ & \\
\hline Variables: & Prepared by: \\
$T / \mathrm{K}=298.15 ;$ Solvent Composition & W. E. Acree, Jr. \\
\hline
\end{tabular}


Experimental Values

Solubility of anthracene in 2,2,4-trimethylmethylpentane + propanone +2 propanol mixtures ${ }^{\mathrm{a}}$

\begin{tabular}{lll}
\hline \hline$x_{2}{ }^{(\mathrm{s})}$ & $x_{3}{ }^{(\mathrm{s})}$ & $c_{1}$ \\
\hline 0.000 & 0.000 & 0.00571 \\
1.000 & 0.000 & 0.00669 \\
0.000 & 1.000 & 0.04353 \\
0.516 & 0.166 & 0.014 \\
0.512 & 0.329 & 0.019 \\
0.456 & 0.316 & 0.018 \\
0.314 & 0.141 & 0.012 \\
0.311 & 0.420 & 0.024 \\
0.309 & 0.557 & 0.031 \\
0.234 & 0.132 & 0.013 \\
0.230 & 0.646 & 0.034 \\
0.162 & 0.487 & 0.037 \\
0.130 & 0.587 & 0.031 \\
0.103 & 0.116 & 0.012 \\
0.102 & 0.345 & 0.020 \\
0.100 & 0.791 & 0.040 \\
0.075 & 0.169 & 0.014 \\
0.073 & 0.768 & 0.039 \\
0.048 & 0.432 & 0.025 \\
0.048 & 0.538 & 0.029 \\
0.048 & 0.272 & 0.014 \\
0.047 & 0.747 & 0.037 \\
${ }^{a} x_{2}{ }^{(\mathrm{s})}:$ initial mole fraction of component 2 in the ternary solvent mixture, $x_{3}{ }^{(\mathrm{s})}:$ \\
initial mole fraction of component 3 in the ternary solvent mixture; $c_{1}:$ molar \\
solubility of the solute. & &
\end{tabular}

\section{Auxiliary Information}

\begin{abstract}
Method/Apparatus/Procedure:
Shaker bath, incubator equipped with temperature controlling system, and an ultraviolet/visible spectrophotometer.

Ternary solvent mixtures were prepared by volume. Excess solute and solvent were placed in sealed containers and allowed to equilibrate for three days at constant temperature. Attainment of equilibrium was verified by several repetitive measurements and by approaching equilibrium from supersaturation. Aliquots of saturated solutions were transferred through a hydrophobic Drapore filters $(0.45 \mu \mathrm{m}$, Millipore, Ireland) into tared volumetric flasks, weighed and diluted with methanol. Molar concentrations were determined by spectrophotometric measurements at $356 \mathrm{~nm}$. The measured molar solubilities were converted to mole fraction solubilities using the measured densities of the saturated solutions.
\end{abstract}

Source and Purity of Chemicals:

(1) $96 \%$, Fluka Chemical Company, recrystallized several times from acetone and ethyl ethanoate.

(2) $99+\%$, Merck Chemical Company, Germany.

(3) Purity and chemical source not specified.

(4) $99.5 \%$, Merck Chemical Company, Germany.

Estimated Error:

Temperature: $\pm 0.2 \mathrm{~K}$

$x_{2}{ }^{(\mathrm{s})}: \pm 0.005$.

$x_{3}{ }^{\text {(s) }}: \pm 0.005$.

$c_{1}: \pm 2.0 \%$ (relative error).

\section{Solubility of Anthracene in Miscellaneous Ternary Solvent Mixtures}

\subsection{Critical evaluation of experimental solubility data}

Jouyban $e t a l . .^{33}$ determined the solubility of anthracene in ternary cyclohexanone + ethyl ethanoate + methanol solvent mixtures at $298.15 \mathrm{~K}$. The authors studied 16 different ternary solvent compositions covering the entire range of mole fraction, plus the three neat organic solvents. There has only been a single experimental determination for the solubility of anthracene in each of the ternary solvent systems studied. The experimental values were evaluated by curve-fitting the measured solubility data in the contributing sub-binary solvent systems to Eq. (5) and then using the ternary solvent version of the basic model to estimate the molar solubility of anthracene dissolved in ternary alkane + alkanone + alcohol solvent mixtures.

The numerical values of the curve-fit parameters for the cyclohexanone (2) + ethyl ethanoate (3) $\left(S_{23,0}=0.835 ; S_{23,1}=\right.$ -1.041 ; and $S_{23,2}=1.209$ ), cyclohexanone (2) + methanol (3) $\left(S_{23,0}=3.601 ; S_{23,1}=-2.715\right.$; and $\left.S_{23,2}=3.790\right)$, and ethyl ethanoate (2) + methanol (3) $\left(S_{23,0}=2.907 ; S_{23,1}=-1.990\right.$; and $S_{23,2}=1.159$ ) were determined through analysis of measured anthracene molar solubilities in the respective binary solvent system. Equation (5) was found to predict the experimental molar solubilities of anthracene in ternary cyclohexanone + ethyl ethanoate + methanol to within an average absolute percent deviation of $20.6 \%$. The large deviations result in part from the large range of molar solubilities covered by the neat organic solvents. Anthracene is over 22 times more soluble in cyclohexanone than in methanol on the molar solubility scale.

\subsection{Anthracene solubility data in ternary cyclohexanone + ethyl ethanoate + methanol solvent mixtures}

\begin{tabular}{|c|c|}
\hline $\begin{array}{l}\text { Components: } \\
\text { (1) Anthracene; } \mathrm{C}_{14} \mathrm{H}_{10} ;[120-12-7] \\
\text { (2) Cyclohexanone; } \mathrm{C}_{6} \mathrm{H}_{10} \mathrm{O} ; \\
\text { [108-94-1] } \\
\text { (3) Ethyl ethanoate; } \mathrm{C}_{4} \mathrm{H}_{8} \mathrm{O}_{2} ; \\
\text { [141-78-6] } \\
\text { (4) Methanol; } \mathrm{CH}_{4} \mathrm{O} ;[67-56-1]\end{array}$ & $\begin{array}{l}\text { Original Measurements: } \\
{ }^{33} \text { A. Jouyban, J. L. Manzoori, } \\
\text { V. Panahi-Azar, J. Soleymani, } \\
\text { M. A. A. Fakhree, A. Shayanfar, } \\
\text { and W. E. Acree, Jr., J. Chem. } \\
\text { Eng. Data 55, 2607 (2010). }\end{array}$ \\
\hline $\begin{array}{l}\text { Variables: } \\
T / \mathrm{K}=298.15 \text {; Solvent Composition }\end{array}$ & $\begin{array}{l}\text { Prepared by: } \\
\text { W. E. Acree, Jr. }\end{array}$ \\
\hline
\end{tabular}


Experimental Values

Solubility of anthracene in cyclohexanone + ethyl ethanoate + methanol mixtures $^{\mathrm{a}}$

\begin{tabular}{lll}
\hline \hline$x_{2}{ }^{(\mathrm{s})}$ & $x_{3}{ }^{(\mathrm{s})}$ & $c_{1}$ \\
\hline 0.000 & 0.000 & 0.006 \\
1.000 & 0.000 & 0.136 \\
0.000 & 1.000 & 0.049 \\
0.044 & 0.047 & 0.011 \\
0.056 & 0.233 & 0.027 \\
0.061 & 0.318 & 0.032 \\
0.084 & 0.702 & 0.042 \\
0.122 & 0.255 & 0.031 \\
0.176 & 0.222 & 0.041 \\
0.202 & 0.282 & 0.052 \\
0.218 & 0.227 & 0.050 \\
0.224 & 0.059 & 0.035 \\
0.246 & 0.128 & 0.042 \\
0.270 & 0.212 & 0.057 \\
0.301 & 0.315 & 0.055 \\
0.308 & 0.064 & 0.052 \\
0.339 & 0.444 & 0.069 \\
0.425 & 0.357 & 0.078 \\
0.690 & 0.090 & 0.105 \\
${ }^{\mathrm{a}} x_{2}(\mathrm{~s})$ initial mole fraction of component 2 in the ternary solvent mixture $x_{3}{ }^{(\mathrm{s})}$ & .
\end{tabular}

${ }^{x_{2}}{ }$ : initial mole fraction of component 2 in the ternary solvent mixture, $x_{3}{ }^{2}$ : solubility of the solute.

\section{Solubility of Phenanthrene in Ternary Alcohol + Alcohol + Alcohol Solvent Mixtures}

\subsection{Critical evaluation of experimental solubility data}

Fakhree et $a l .{ }^{34}$ reported the solubility of phenanthrene in five different ternary solvent mixtures containing various combinations of methanol, ethanol, 1-propanol, 2-propanol, and 1-butanol. There has only been a single experimental determination for the solubility of phenanthrene in each of the five ternary solvent systems studied. In the absence of replicate independent measurements, the experimental values were evaluated by curve-fitting the measured binary solvent solubility data to Eq. (4), and then using the ternary solvent version of the basic model to estimate the mole fraction solubility of phenanthrene dissolved in ternary alcohol + alcohol + alcohol solvent mixtures.

The calculated $S_{23, i}$ parameters for all of the contributing binary alcohol + alcohol solvent systems studied are summarized in Table 9, along with the average absolute deviation between the experimental and back-calculated mole fraction solubilities. The predictive ability of Eq. (5) is given in

TABLE 9. Combined NIBS/Redlich-Kister $S_{23, i}$ parameters for mathematical representation of phenanthrene molar solubilities in binary solvent mixtures

\section{Auxiliary Information}

\section{Method/Apparatus/Procedure:}

Shaker bath, incubator equipped with temperature controlling system, and an ultraviolet/visible spectrophotometer.

Ternary solvent mixtures were prepared by mass. Excess solute and solvent were placed in sealed containers and allowed to equilibrate for three days at constant temperature. Attainment of equilibrium was verified by several repetitive measurements and by approaching equilibrium from supersaturation. Aliquots of saturated solutions were transferred through a hydrophobic Drapore filters $(0.45 \mu \mathrm{m}$, Millipore, Ireland $)$ into tared volumetric flasks, weighed and diluted with methanol. Molar concentrations were determined by spectrophotometric measurements at $356 \mathrm{~nm}$. The measured molar solubilities were converted to mole fraction solubilities using the measured densities of the saturated solutions.

\section{Source and Purity of Chemicals:}

(1) $96 \%$, Fluka Chemical Company, recrystallized several times from acetone and ethyl ethanoate.

(2) $99 \%$, Merck Chemical Company, Germany.

(3) $99.5 \%$, Merck Chemical Company, Germany.

(4) $99.5 \%$, Merck Chemical Company, Germany.

\section{Estimated Error:}

Temperature: $\pm 0.2 \mathrm{~K}$

$x_{2}{ }^{(s)}: \pm 0.005$.

$x_{3}{ }^{(\mathrm{s})}: \pm 0.005$.

$c_{1}: \pm 2.0 \%$ (relative error).

\begin{tabular}{|c|c|c|}
\hline Solvent (2) + solvent (3) & $S_{23, i}{ }^{\mathrm{a}}$ & $\% \operatorname{Dev}^{b}$ \\
\hline Ethanol (2) + methanol (3) & $\begin{array}{r}0.450 \\
-0.192 \\
-0.535\end{array}$ & 1.5 \\
\hline Methanol (2) + 1-propanol (3) & $\begin{array}{l}1.079 \\
0.847 \\
0.864\end{array}$ & 1.5 \\
\hline Methanol (2) + 1-butanol (3) & $\begin{array}{l}1.255 \\
0.604 \\
0.356\end{array}$ & 0.8 \\
\hline Ethanol (2) + 1-propanol (3) & $\begin{array}{r}0.260 \\
-0.165 \\
0.065\end{array}$ & 0.4 \\
\hline Ethanol (2) + 1-butanol (3) & $\begin{array}{r}0.339 \\
0.346 \\
-0.244\end{array}$ & 1.1 \\
\hline 1-Propanol (2) + 1-butanol (3) & $\begin{array}{r}-0.018 \\
-0.191 \\
0.066\end{array}$ & 0.8 \\
\hline Methanol (2) + 2-propanol (3) & $\begin{array}{r}0.741 \\
0.400 \\
-0.063\end{array}$ & 1.7 \\
\hline Ethanol (2) + 2-propanol (3) & $\begin{array}{r}0.238 \\
-0.141 \\
0.106\end{array}$ & 1.5 \\
\hline 1-Propanol (2) + 2-propanol (3) & $\begin{array}{r}0.037 \\
0.156 \\
-0.606\end{array}$ & 0.6 \\
\hline 1-Butanol (2) + 2-propanol (3) & $\begin{array}{r}0.116 \\
0.232 \\
-0.263\end{array}$ & 1.5 \\
\hline
\end{tabular}

${ }^{a}$ Combined NIBS/Redlich-Kister curve-fit parameters are ordered as $S_{23,0}$, $S_{23,1}$, and $S_{23,2}$.

${ }^{\mathrm{b}} \operatorname{Dev}(\%)=(100 / N) \Sigma\left|\left[c_{1}{ }^{\exp }-c_{1}{ }^{\mathrm{cal}}\right] / c_{1}{ }^{\exp }\right|$. 
TABLE 10. Summarized comparison between experimental solubilities of phenanthrene in ternary alcohol + alcohol + alcohol solvent mixtures and predicted values based on Eq. (5)

\begin{tabular}{lc}
\hline \hline Ternary solvent mixture & $\%$ Dev. $^{\text {a }}$ \\
\hline Ethanol + 1-propanol + methanol & 3.4 \\
Ethanol + 1-propanol + 2-propanol & 2.4 \\
1-Butanol + methanol + 2-propanol & 10.4 \\
1-Butanol + ethanol + 1-propanol & 2.9 \\
1-Butanol + 1-propanol + methanol & 4.6 \\
\hline
\end{tabular}

${ }^{\mathrm{a}} \operatorname{Dev}(\%)=(100 / N) \sum\left|\left[x_{1}{ }^{\text {exp }}-x_{1}{ }^{\text {cal }}\right] / x_{1}{ }^{\text {exp }}\right|$.

Table 10 for the five ternary alcohol + alcohol + alcohol solvent systems. Examination of the numerical entries in the last column of Table 10 reveals that Eq. (5) does provide reasonable predictions for how the measured molar solubilities vary with ternary solvent composition. Average relative percent deviations range from $2.4 \%$ to $10.4 \%$, with the largest deviation of $10.4 \%$ being observed in the ternary 1-butanol + methanol +2 -propanol system. Equation (5) underestimates the observed solubility in this ternary system.

The experimental phenanthrene solubility data for ternary alcohol + alcohol + alcohol solvent mixtures are listed in Sec. 10.2.

\subsection{Phenanthrene solubility data in ternary alcohol + alcohol + alcohol solvent mixtures}

\begin{tabular}{ll}
\hline \hline Components: & Original Measurements: \\
(1) Phenanthrene; $\mathrm{C}_{14} \mathrm{H}_{10} ;[85-01-8]$ & ${ }^{34} \mathrm{M}$. A. A. Fakhree, W. E. Acree, \\
(2) Ethanol; $\mathrm{C}_{2} \mathrm{H}_{6} \mathrm{O} ;[64-17-5]$ & Jr., and A. Jouyban, Ind. Eng. \\
(3) 1-Propanol; $\mathrm{C}_{3} \mathrm{H}_{8} \mathrm{O} ;[71-23-8]$ & Chem. Res. 49, 6238 (2010). \\
(4) Methanol; $\mathrm{CH}_{4} \mathrm{O} ;[67-56-1]$ & \\
\hline Variables: & Prepared by: \\
$T / \mathrm{K}=298.15 ;$ Solvent Composition & W. E. Acree, Jr. \\
\hline
\end{tabular}

\section{Experimental Values}

Solubility of phenanthrene in ethanol +1 -propanol + methanol mixtures $^{\mathrm{a}}$

\begin{tabular}{lcc}
\hline \hline$x_{2}{ }^{(\mathrm{s})}$ & $x_{3}{ }^{(\mathrm{s})}$ & $c_{1}$ \\
\hline 0.000 & 0.000 & 0.133 \\
1.000 & 0.000 & 0.187 \\
0.000 & 1.000 & 0.174 \\
0.783 & 0.076 & 0.186 \\
0.700 & 0.156 & 0.185 \\
0.656 & 0.073 & 0.185 \\
0.574 & 0.149 & 0.186 \\
0.523 & 0.326 & 0.192 \\
0.489 & 0.229 & 0.189 \\
0.433 & 0.068 & 0.177 \\
0.428 & 0.418 & 0.195
\end{tabular}

\begin{tabular}{lcc}
0.333 & 0.065 & 0.173 \\
0.306 & 0.399 & 0.201 \\
0.287 & 0.299 & 0.186 \\
0.225 & 0.613 & 0.192 \\
0.173 & 0.203 & 0.180 \\
0.155 & 0.061 & 0.160 \\
0.115 & 0.719 & 0.196 \\
0.107 & 0.584 & 0.197 \\
0.094 & 0.366 & 0.188 \\
0.088 & 0.275 & 0.174 \\
0.079 & 0.123 & 0.168 \\
0.075 & 0.059 & 0.158 \\
\hline${ }^{a} x_{2}{ }^{(s)}:$ initial mole fraction of component 2 in the ternary solvent mixture, $x_{3}{ }^{(s)}:$ \\
initial mole fraction of component 3 in the ternary solvent mixture; $c_{1}:$ molar \\
solubility of the solute.
\end{tabular}

\section{Auxiliary Information}

\section{Method/Apparatus/Procedure:}

Shaker bath, incubator equipped with temperature controlling system, and an ultraviolet/visible spectrophotometer.

Binary solvent mixtures were prepared by volume. Excess solute and solvent were placed in sealed containers and allowed to equilibrate for three days at constant temperature. Attainment of equilibrium was verified by several repetitive measurements and by approaching equilibrium from supersaturation. Aliquots of saturated solutions were transferred through a hydrophobic Drapore filters ( $0.45 \mu \mathrm{m}$, Millipore, Ireland) into tared volumetric flasks, weighed and diluted with propanone. Molar concentrations were determined by spectrophotometric measurements at $345 \mathrm{~nm}$. The measured molar solubilities were converted to mole fraction solubilities using the measured densities of the saturated solutions.

\section{Source and Purity of Chemicals:}

(1) $98 \%$, Merck Chemical Company, Germany, was recrystallized several times from acetone.

(2) $99.9 \%$, Merck Chemical Company.

(3) $99.5 \%$, Merck Chemical Company.

(4) $99.5 \%$, Merck Chemical Company.

Estimated Error:

Temperature: $\pm 0.2 \mathrm{~K}$.

$x_{2}{ }^{(\mathrm{s})}: \pm 0.001$.

$x_{3}{ }^{(\mathrm{s})}: \pm 0.001$

$c_{1}: \pm 2.7 \%$ (relative error)

\begin{tabular}{ll}
\hline \hline Components: & Original Measurements: \\
(1) Phenanthrene; $\mathrm{C}_{14} \mathrm{H}_{10} ;[85-01-8]$ & ${ }^{34} \mathrm{M}$. A. A. Fakhree, W. E. \\
(2) Ethanol; $\mathrm{C}_{2} \mathrm{H}_{6} \mathrm{O} ;[64-17-5]$ & Acree, Jr., and A. Jouyban, \\
(3) 1-Propanol; $\mathrm{C}_{3} \mathrm{H}_{8} \mathrm{O} ;[71-23-8]$ & Ind. Eng. Chem. Res. 49, 6238 \\
(4) 2-Propanol; $\mathrm{C}_{3} \mathrm{H}_{8} \mathrm{O} ;[67-63-0]$ & $(2010)$. \\
\hline Variables: & Prepared by: \\
$T / \mathrm{K}=298.15 ;$ Solvent Composition & W. E. Acree, Jr. \\
\hline
\end{tabular}


Experimental Values

Solubility of phenanthrene in ethanol +1 -propanol +2 -propanol mixtures ${ }^{\mathrm{a}}$

\begin{tabular}{lcc}
\hline \hline$x_{2}{ }^{(\mathrm{s})}$ & $x_{3}{ }^{(\mathrm{s})}$ & $c_{1}$ \\
\hline 0.000 & 0.000 & 0.124 \\
1.000 & 0.000 & 0.187 \\
0.000 & 1.000 & 0.174 \\
0.838 & 0.082 & 0.183 \\
0.751 & 0.167 & 0.179 \\
0.752 & 0.084 & 0.175 \\
0.660 & 0.172 & 0.175 \\
0.563 & 0.351 & 0.182 \\
0.564 & 0.264 & 0.179 \\
0.566 & 0.088 & 0.165 \\
0.462 & 0.450 & 0.188 \\
0.465 & 0.091 & 0.161 \\
0.356 & 0.463 & 0.178 \\
0.357 & 0.371 & 0.164 \\
0.243 & 0.664 & 0.177 \\
0.245 & 0.287 & 0.154 \\
0.246 & 0.096 & 0.144 \\
0.125 & 0.780 & 0.178 \\
0.125 & 0.684 & 0.170 \\
0.126 & 0.490 & 0.159 \\
0.126 & 0.393 & 0.164 \\
0.126 & 0.197 & 0.148 \\
0.127 & 0.099 & 0.142 \\
\hline${ }_{x_{2}{ }^{(\mathrm{s})}: \text { initial mole fraction of component } 2 \text { in the ternary solvent mixture, } x_{3}{ }^{(\mathrm{s})}:}$ \\
initial mole fraction of component 3 in the ternary solvent mixture; $c_{1}:$ molar \\
solubility of the solute. & &
\end{tabular}

\section{Auxiliary Information}

\section{Method/Apparatus/Procedure:}

Shaker bath, incubator equipped with temperature controlling system, and an ultraviolet/visible spectrophotometer.

Binary solvent mixtures were prepared by volume. Excess solute and solvent were placed in sealed containers and allowed to equilibrate for three days at constant temperature. Attainment of equilibrium was verified by several repetitive measurements and by approaching equilibrium from supersaturation. Aliquots of saturated solutions were transferred through a hydrophobic Drapore filters $(0.45 \mu \mathrm{m}$, Millipore, Ireland $)$ into tared volumetric flasks, weighed and diluted with propanone. Molar concentrations were determined by spectrophotometric measurements at $345 \mathrm{~nm}$. The measured molar solubilities were converted to mole fraction solubilities using the measured densities of the saturated solutions.

\section{Source and Purity of Chemicals:}

(1) $98 \%$, Merck Chemical Company, Germany, was recrystallized several times from acetone.

(2) $99.5 \%$, Merck Chemical Company.

(3) $99.5 \%$, Merck Chemical Company.

(4) $99.5 \%$, Merck Chemical Company.

\section{Estimated Error:}

Temperature: $\pm 0.2 \mathrm{~K}$.

$x_{2}{ }^{(\mathrm{s})}: \pm 0.001$.

$x_{3}{ }^{(s)}: \pm 0.001$.

$c_{1}: \pm 2.7 \%$ (relative error).

\begin{tabular}{ll}
\hline \hline Components: & Original Measurements: \\
(1) Phenanthrene; $\mathrm{C}_{14} \mathrm{H}_{10} ;[85-01-8]$ & ${ }^{34} \mathrm{M}$. A. A. Fakhree, W. E. Acree, \\
(2) 1-Butanol; $\mathrm{C}_{4} \mathrm{H}_{10} \mathrm{O} ;[71-36-3]$ & Jr., and A. Jouyban, Ind. Eng. \\
(3) Methanol; $\mathrm{CH}_{4} \mathrm{O} ;[67-56-1]$ & Chem. Res. 49, 6238 (2010). \\
(4) 2-Propanol; $\mathrm{C}_{3} \mathrm{H}_{8} \mathrm{O} ;[67-63-0]$ & \\
\hline Variables: & Prepared by: \\
$T / \mathrm{K}=298.15 ;$ Solvent Composition & W. E. Acree, Jr. \\
\hline
\end{tabular}

Experimental Values

Solubility of phenanthrene in 1-butanol + methanol +2 -propanol mixtures ${ }^{\mathrm{a}}$

\begin{tabular}{llc}
\hline \hline$x_{2}{ }^{(\mathrm{s})}$ & $x_{3}{ }^{(\mathrm{s})}$ & $c_{1}$ \\
\hline 0.000 & 0.000 & 0.124 \\
1.000 & 0.000 & 0.188 \\
0.000 & 1.000 & 0.133 \\
0.698 & 0.197 & 0.234 \\
0.550 & 0.356 & 0.242 \\
0.601 & 0.194 & 0.222 \\
0.328 & 0.594 & 0.214 \\
0.381 & 0.345 & 0.217 \\
0.415 & 0.188 & 0.195 \\
0.242 & 0.685 & 0.214 \\
0.327 & 0.185 & 0.189 \\
0.206 & 0.466 & 0.200 \\
0.105 & 0.832 & 0.185 \\
0.111 & 0.755 & 0.200 \\
0.118 & 0.669 & 0.199 \\
0.135 & 0.459 & 0.185 \\
0.158 & 0.179 & 0.172 \\
0.049 & 0.892 & 0.172 \\
0.052 & 0.823 & 0.177 \\
0.058 & 0.661 & 0.184 \\
0.062 & 0.564 & 0.183 \\
0.072 & 0.325 & 0.172 \\
0.078 & 0.176 & 0.162 \\
\hline$x_{2}(\mathrm{~s} i$ & &
\end{tabular}

$\frac{}{{ }^{\mathrm{a}} x_{2}{ }^{(\mathrm{s})} \text { : initial mole fraction of component } 2 \text { in the ternary solvent mixture, } x_{3}{ }^{(\mathrm{s})} \text { : }}$ initial mole fraction of component 3 in the ternary solvent mixture; $c_{1}$ : molar solubility of the solute.

\section{Auxiliary Information}

Method/Apparatus/Procedure:

Shaker bath, incubator equipped with temperature controlling system, and an ultraviolet/visible spectrophotometer.

Binary solvent mixtures were prepared by volume. Excess solute and solvent were placed in sealed containers and allowed to equilibrate for three days at constant temperature. Attainment of equilibrium was verified by several repetitive measurements and by approaching equilibrium from supersaturation. Aliquots of saturated solutions were transferred through a hydrophobic Drapore filters $(0.45 \mu \mathrm{m}$, Millipore, Ireland $)$ into tared volumetric flasks, weighed and diluted with propanone. Molar concentrations were determined by spectrophotometric measurements at $345 \mathrm{~nm}$. The measured molar solubilities were converted to mole fraction solubilities using the measured densities of the saturated solutions.

Source and Purity of Chemicals:

(1) $98 \%$, Merck Chemical Company, Germany, was recrystallized several times from acetone.

(2) $99.5 \%$, Merck Chemical Company.

(3) $99.5 \%$, Merck Chemical Company.

(4) $99.5 \%$, Merck Chemical Company. 
Estimated Error:

Temperature: $\pm 0.2 \mathrm{~K}$

$x_{2}{ }^{(\mathrm{s})}: \pm 0.001$.

$x_{3}{ }^{(\mathrm{s})}: \pm 0.001$.

$c_{1}: \pm 2.7 \%$ (relative error)

\begin{tabular}{|c|c|}
\hline $\begin{array}{l}\text { Components: } \\
\text { (1) Phenanthrene; } \mathrm{C}_{14} \mathrm{H}_{10} ;[85-01-8] \\
\text { (2) 1-Butanol; } \mathrm{C}_{4} \mathrm{H}_{10} \mathrm{O} ;[71-36-3] \\
\text { (3) Ethanol; } \mathrm{C}_{2} \mathrm{H}_{6} \mathrm{O} ;[64-17-5] \\
\text { (4) 1-Propanol; } \mathrm{C}_{3} \mathrm{H}_{8} \mathrm{O} ;[71-23-8]\end{array}$ & $\begin{array}{l}\text { Original Measurements: } \\
{ }^{34} \text { M. A. A. Fakhree, W. E. Acree, } \\
\text { Jr., and A. Jouyban, Ind. Eng. } \\
\text { Chem. Res. 49, } 6238 \text { (2010). }\end{array}$ \\
\hline $\begin{array}{l}\text { Variables: } \\
T / \mathrm{K}=298.15 \text {; Solvent Composition } \\
\end{array}$ & $\begin{array}{l}\text { Prepared by: } \\
\text { W. E. Acree, Jr. }\end{array}$ \\
\hline
\end{tabular}

\section{Experimental Values}

Solubility of phenanthrene in 1-butanol + ethanol +1 -propanol mixtures ${ }^{\mathrm{a}}$

\begin{tabular}{lcc}
\hline \hline$x_{2}{ }^{(\mathrm{s})}$ & $x_{3}{ }^{(\mathrm{s})}$ & $c_{1}$ \\
\hline 0.000 & 0.000 & 0.174 \\
1.000 & 0.000 & 0.188 \\
0.000 & 1.000 & 0.187 \\
0.741 & 0.145 & 0.199 \\
0.616 & 0.276 & 0.203 \\
0.636 & 0.142 & 0.201 \\
0.400 & 0.502 & 0.207 \\
0.424 & 0.266 & 0.202 \\
0.436 & 0.137 & 0.200 \\
0.306 & 0.600 & 0.201 \\
0.342 & 0.134 & 0.201 \\
0.238 & 0.373 & 0.199 \\
0.141 & 0.773 & 0.194 \\
0.144 & 0.679 & 0.196 \\
0.148 & 0.580 & 0.195 \\
0.156 & 0.367 & 0.198 \\
0.165 & 0.129 & 0.190 \\
0.068 & 0.849 & 0.189 \\
0.069 & 0.761 & 0.188 \\
0.073 & 0.571 & 0.195 \\
0.075 & 0.468 & 0.197 \\
0.079 & 0.247 & 0.189 \\
0.081 & 0.127 & 0.188 \\
\hline$x_{2}{ }^{(\mathrm{s})}:$ initial mole fraction of component 2 in the ternary solvent mixture, $x_{3}{ }^{(\mathrm{s})}$ & \\
initial mole fraction of component 3 in the ternary solvent mixture; $c_{1}:$ molar \\
solubility of the solute. & &
\end{tabular}

\section{Auxiliary Information}

\section{Method/Apparatus/Procedure:}

Shaker bath, incubator equipped with temperature controlling system, and an ultraviolet/visible spectrophotometer.

Binary solvent mixtures were prepared by volume. Excess solute and solvent were placed in sealed containers and allowed to equilibrate for three days at constant temperature. Attainment of equilibrium was verified by several repetitive measurements and by approaching equilibrium from supersaturation. Aliquots of saturated solutions were transferred through a hydrophobic Drapore filters ( $0.45 \mu \mathrm{m}$, Millipore, Ireland) into tared volumetric flasks, weighed and diluted with propanone. Molar concentrations were determined by spectrophotometric measurements at $345 \mathrm{~nm}$. The measured molar solubilities were converted to mole fraction solubilities using the measured densities of the saturated solutions.
Source and Purity of Chemicals:

(1) $98 \%$, Merck Chemical Company, Germany, was recrystallized several times from acetone.

(2) $99.5 \%$, Merck Chemical Company.

(3) $99.5 \%$, Merck Chemical Company.

(4) $99.5 \%$, Merck Chemical Company.

\section{Estimated Error:}

Temperature: $\pm 0.2 \mathrm{~K}$.

$x_{2}{ }^{(\mathrm{s})}: \pm 0.001$

$x_{3}{ }^{(s)}: \pm 0.001$.

$c_{1}: \pm 2.7 \%$ (relative error).

\begin{tabular}{|c|c|}
\hline $\begin{array}{l}\text { Components: } \\
\text { (1) Phenanthrene; } \mathrm{C}_{14} \mathrm{H}_{10} ;[85-01-8] \\
\text { (2) 1-Butanol; } \mathrm{C}_{4} \mathrm{H}_{10} \mathrm{O} ;[71-36-3] \\
\text { (3) 1-Propanol; } \mathrm{C}_{3} \mathrm{H}_{8} \mathrm{O} ;[71-23-8] \\
\text { (4) Methanol; } \mathrm{CH}_{4} \mathrm{O} ;[67-56-1]\end{array}$ & $\begin{array}{l}\text { Original Measurements: } \\
{ }^{34} \text { M. A. A. Fakhree, W. E. Acree, } \\
\text { Jr., and A. Jouyban, Ind. Eng. } \\
\text { Chem. Res. 49, } 6238 \text { (2010). }\end{array}$ \\
\hline $\begin{array}{l}\text { Variables: } \\
T / \mathrm{K}=298.15 ; \text { Solvent Composition }\end{array}$ & $\begin{array}{l}\text { Prepared by: } \\
\text { W. E. Acree, Jr. }\end{array}$ \\
\hline
\end{tabular}

Experimental Values

Solubility of phenanthrene in 1-butanol +1 -propanol + methanol mixtures ${ }^{\mathrm{a}}$

\begin{tabular}{llc}
\hline \hline$x_{2}^{(\mathrm{s})}$ & $x_{3}{ }^{(\mathrm{s})}$ & $c_{1}$ \\
\hline 0.000 & 0.000 & 0.133 \\
1.000 & 0.000 & 0.188 \\
0.000 & 1.000 & 0.174 \\
0.697 & 0.107 & 0.221 \\
0.598 & 0.209 & 0.225 \\
0.549 & 0.096 & 0.223 \\
0.411 & 0.403 & 0.214 \\
0.379 & 0.278 & 0.221 \\
0.327 & 0.080 & 0.227 \\
0.323 & 0.494 & 0.216 \\
0.242 & 0.074 & 0.219 \\
0.204 & 0.333 & 0.218 \\
0.156 & 0.668 & 0.210 \\
0.134 & 0.410 & 0.217 \\
0.118 & 0.216 & 0.211 \\
0.111 & 0.136 & 0.202 \\
0.105 & 0.064 & 0.192 \\
0.077 & 0.750 & 0.208 \\
0.071 & 0.608 & 0.206 \\
0.062 & 0.378 & 0.229 \\
0.058 & 0.284 & 0.205 \\
0.052 & 0.127 & 0.187 \\
0.049 & 0.060 & 0.178 \\
\hline$x_{2}(\mathrm{sin}$ & &
\end{tabular}

${ }^{\mathrm{a}}{ }_{x_{2}}{ }^{(\mathrm{s})}$ : initial mole fraction of component 2 in the ternary solvent mixture, $x_{3}{ }^{(\mathrm{s})}$ : initial mole fraction of component 3 in the ternary solvent mixture; $c_{1}$ : molar solubility of the solute. 


\section{Auxiliary Information}

\section{Method/Apparatus/Procedure:}

Shaker bath, incubator equipped with temperature controlling system, and an ultraviolet/visible spectrophotometer.

Binary solvent mixtures were prepared by volume. Excess solute and solvent were placed in sealed containers and allowed to equilibrate for three days at constant temperature. Attainment of equilibrium was verified by several repetitive measurements and by approaching equilibrium from supersaturation. Aliquots of saturated solutions were transferred through a hydrophobic Drapore filters $(0.45 \mu \mathrm{m}$, Millipore, Ireland) into tared volumetric flasks, weighed and diluted with propanone. Molar concentrations were determined by spectrophotometric measurements at $345 \mathrm{~nm}$. The measured molar solubilities were converted to mole fraction solubilities using the measured densities of the saturated solutions.

Source and Purity of Chemicals:

(1) $98 \%$, Merck Chemical Company, Germany, was recrystallized several times from acetone.

(2) $99.5 \%$, Merck Chemical Company.

(3) $99.5 \%$, Merck Chemical Company.

(4) $99.5 \%$, Merck Chemical Company.

Estimated Error:

Temperature: $\pm 0.2 \mathrm{~K}$.

$x_{2}{ }^{(\mathrm{s})}: \pm 0.001$.

$x_{3}{ }^{(\mathrm{s})}: \pm 0.001$.

$c_{1}: \pm 2.7 \%$ (relative error).

\section{Solubility of Pyrene in Ternary Alkane + Alkane + Alcohol Solvent Mixtures}

\subsection{Critical evaluation of experimental solubility data}

Debase and Acree ${ }^{31}$ determined the solubility of pyrene in ternary cyclohexane + heptane + alcohol solvent mixtures at $299.15 \mathrm{~K}$ based on spectroscopic absorbance measurements. The five alcohol cosolvents used were 1-propanol, 2-propanol, 1-butanol, 2-butanol, and 2-methyl-1-propanol. There has only been a single experimental determination for the solubility of pyrene in each of the ternary solvent systems studied. The experimental values were evaluated by curve-fitting the measured solubility data for the sub-binary solvent systems to the Combined NIBS/Redlich-Kister equation, and then using Eq. (2) to estimate the mole fraction solubility of pyrene dissolved in ternary alkane + alkane + alcohol solvent mixtures.

The calculated $S_{23, i}$ parameters for all of the contributing binary alkane + alkane and alkane + alcohol solvent systems studied are summarized in Table 2, along with the average absolute deviation between the experimental and back-calculated mole fraction solubilities. The predictive ability of Eq. (2) is given in Table 11 for the five ternary alcohol + alcohol + alkoxyalcohol solvent systems. Examination of the numerical entries in the last column of Table 11 reveals that Eq. (2) does provide accurate estimations for how the measured mole fraction solubilities vary with ternary solvent composition. Average percent deviations range from $1.0 \%$ to $1.6 \%$, suggesting that there are no obvious outliers in an individual ternary solvent data set. None of the experimental data points was flagged as an outlier.
TABLE 11. Summarized comparison between experimental solubilities of pyrene in ternary alkane + alkane + alcohol solvent mixtures and predicted values based on Eq. (2)

\begin{tabular}{lc}
\hline \hline Ternary solvent mixture & $\%$ Dev. \\
\hline Heptane + cyclohexane + 1-propanol & 1.63 \\
Heptane + cyclohexane + 2-propanol & 1.13 \\
Heptane + cyclohexane + 1-butanol & 1.00 \\
Heptane + cyclohexane + 2-butanol & 1.06 \\
Heptane + cyclohexane + 2-methyl-1-propanol & 1.52 \\
\hline${ }^{a}$ Dev $(\%)=(100 / N) \Sigma$
\end{tabular}

${ }^{\mathrm{a}} \operatorname{Dev}(\%)=(100 / N) \Sigma\left|\left[x_{1}{ }^{\text {exp }}-x_{1}{ }^{\mathrm{cal}}\right] / x_{1}{ }^{\mathrm{exp}}\right|$.

The experimental pyrene solubility data for ternary heptane + cyclohexane + alcohol solvent mixtures are reported in Sec. 11.2.

\subsection{Pyrene solubility data in ternary heptane + cyclohexane + alcohol solvent mixtures}

\begin{tabular}{|c|c|}
\hline $\begin{array}{l}\text { Components: } \\
\text { (1) Pyrene; } \mathrm{C}_{16} \mathrm{H}_{10} ;[129-00-0] \\
\text { (2) Cyclohexane; } \mathrm{C}_{6} \mathrm{H}_{12} ;[110-82-7] \\
\text { (3) Heptane; } \mathrm{C}_{7} \mathrm{H}_{16} ;[142-82-5] \\
\text { (4) 1-Propanol; } \mathrm{C}_{3} \mathrm{H}_{8} \mathrm{O} ;[71-23-8]\end{array}$ & $\begin{array}{l}\text { Original Measurements: } \\
{ }^{31} \text { E. M. Debase and W. E. Acree, } \\
\text { Jr., J. Chem. Eng. Data 46, } 1297 \\
(2001) \text {. }\end{array}$ \\
\hline $\begin{array}{l}\text { Variables: } \\
T / \mathrm{K}=299.15 \text {; Solvent Composition }\end{array}$ & $\begin{array}{l}\text { Prepared by: } \\
\text { W. E. Acree, Jr. }\end{array}$ \\
\hline
\end{tabular}

Experimental Values

Solubility of pyrene in cyclohexane + heptane +1 -propanol mixtures ${ }^{\mathrm{a}}$

\begin{tabular}{lcccc}
\hline \hline$x_{2}{ }^{(\mathrm{s})}$ & $x_{4}{ }^{(\mathrm{s})}$ & $x_{2}$ & $x_{4}$ & $x_{1}$ \\
\hline 0.0000 & 0.0000 & 0.0000 & 0.0000 & 0.01102 \\
1.0000 & 0.0000 & 0.9890 & 0.0000 & 0.01100 \\
0.0000 & 1.0000 & 0.0000 & 0.9957 & 0.00426 \\
0.3186 & 0.4484 & 0.3153 & 0.4437 & 0.01048 \\
0.6746 & 0.2184 & 0.6664 & 0.2157 & 0.01218 \\
0.2561 & 0.3649 & 0.2533 & 0.3609 & 0.01107 \\
0.4769 & 0.3407 & 0.4715 & 0.3368 & 0.01132 \\
0.0869 & 0.7921 & 0.0863 & 0.7867 & 0.00688 \\
0.1601 & 0.7809 & 0.1590 & 0.7755 & 0.00682 \\
0.6594 & 0.2700 & 0.6518 & 0.2669 & 0.01147 \\
0.5280 & 0.1693 & 0.5216 & 0.1672 & 0.01216 \\
0.4296 & 0.5026 & 0.4254 & 0.4977 & 0.00984 \\
0.3286 & 0.6083 & 0.3221 & 0.5962 & 0.00879 \\
0.1701 & 0.2510 & 0.1681 & 0.2481 & 0.01148 \\
0.1227 & 0.7897 & 0.1219 & 0.7844 & 0.00677 \\
0.2147 & 0.6229 & 0.2155 & 0.6175 & 0.00868 \\
0.2221 & 0.1805 & 0.2195 & 0.1784 & 0.01186 \\
0.6989 & 0.1497 & 0.6904 & 0.1479 & 0.01221 \\
0.1097 & 0.3248 & 0.1085 & 0.3212 & 0.01109 \\
0.4284 & 0.1719 & 0.4232 & 0.1698 & 0.01214 \\
0.0990 & 0.5488 & 0.0989 & 0.5483 & 0.00946 \\
0.0905 & 0.6468 & 0.0904 & 0.6462 & 0.00851 \\
\hline${ }^{(\mathrm{s})}:$ & &
\end{tabular}

${ }^{\mathrm{a}} x_{2}{ }^{(\mathrm{s})}$ : initial mole fraction of component 2 in the ternary solvent mixture, $x_{4}{ }^{(\mathrm{s})}$ : initial mole fraction of component 4 in the ternary solvent mixture; $x_{1}$ : mole fraction solubility of the solute; $x_{2}$ : mole fraction of component 2 in the quaternary solution; $x_{4}$ : mole fraction of component 4 in the quaternary solution. 


\section{Auxiliary Information}

\section{Method/Apparatus/Procedure:}

Constant-temperature bath, calorimetric thermometer, and an ultraviolet/ visible spectrophotometer.

Ternary solvent mixtures were prepared by mass. Excess solute and solvent were placed in amber glass bottles and allowed to equilibrate for several days at constant temperature. Attainment of equilibrium was verified by several repetitive measurements and by approaching equilibrium from supersaturation. Aliquots of saturated solutions were transferred through a coarse filter into volumetric flasks, weighed and diluted with methanol. Molar concentrations were determined by spectrophotometric measurements at $372 \mathrm{~nm}$.

\section{Source and Purity of Chemicals:}

(1) $98 \%$, Aldrich Chemical Company, Milwaukee, WI, USA, recrystallized three times from anhydrous methanol.

(2) $99.9 \%$, HPLC grade, Aldrich Chemical Company.

(3) $99 \%$, HPLC grade, Aldrich Chemical Company.

(4) $99+\%$, anhydrous, Aldrich Chemical Company.

Components $2-4$ were stored over molecular sieves and distilled shortly before use.

\section{Estimated Error:}

Temperature: $\pm 0.1 \mathrm{~K}$

$x_{2}{ }^{(\mathrm{s})}: \pm 0.0001$.

$x_{4}{ }^{(\mathrm{s})}: \pm 0.0001$

$x_{1}: \pm 1.5 \%$ (relative error).

\begin{tabular}{|c|c|}
\hline $\begin{array}{l}\text { Components: } \\
\text { (1) Pyrene; } \mathrm{C}_{16} \mathrm{H}_{10} ;[129-00-0] \\
\text { (2) Cyclohexane; } \mathrm{C}_{6} \mathrm{H}_{12} ;[110-82-7] \\
\text { (3) Heptane; } \mathrm{C}_{7} \mathrm{H}_{16} ;[142-82-5] \\
\text { (4) 2-Propanol; } \mathrm{C}_{3} \mathrm{H}_{8} \mathrm{O} ;[67-63-0]\end{array}$ & $\begin{array}{l}\text { Original Measurements: } \\
{ }^{31} \text { E. M. Debase and W. E. Acree, } \\
\text { Jr., J. Chem. Eng. Data 46, } 1297 \\
\text { (2001). }\end{array}$ \\
\hline $\begin{array}{l}\text { Variables: } \\
T / \mathrm{K}=299.15 ; \text { Solvent Composition }\end{array}$ & $\begin{array}{l}\text { Prepared by: } \\
\text { W. E. Acree, Jr. }\end{array}$ \\
\hline
\end{tabular}

Experimental Values

Solubility of pyrene in cyclohexane + heptane +2 -propanol mixtures ${ }^{\mathrm{a}}$

\begin{tabular}{lcccc}
\hline \hline$x_{2}{ }^{(\mathrm{s})}$ & $x_{4}{ }^{(\mathrm{s})}$ & $x_{2}$ & $x_{4}$ & $x_{1}$ \\
\hline 0.0000 & 0.0000 & 0.0000 & 0.0000 & 0.01102 \\
1.0000 & 0.0000 & 0.9890 & 0.0000 & 0.01100 \\
0.0000 & 1.0000 & 0.0000 & 0.9971 & 0.00290 \\
0.3148 & 0.4546 & 0.3117 & 0.4502 & 0.00973 \\
0.6816 & 0.2110 & 0.6737 & 0.2085 & 0.01164 \\
0.2581 & 0.3624 & 0.2554 & 0.3586 & 0.01048 \\
0.4789 & 0.3384 & 0.4738 & 0.3348 & 0.01067 \\
0.0848 & 0.7960 & 0.0843 & 0.7917 & 0.00545 \\
0.1614 & 0.7801 & 0.1605 & 0.7758 & 0.00557 \\
0.6539 & 0.2693 & 0.6466 & 0.2663 & 0.01121 \\
0.5202 & 0.1628 & 0.5141 & 0.1609 & 0.01177 \\
0.4378 & 0.4923 & 0.4338 & 0.4878 & 0.00923 \\
0.3366 & 0.5946 & 0.3340 & 0.5899 & 0.00787 \\
0.1794 & 0.2470 & 0.1774 & 0.2442 & 0.01115 \\
0.1220 & 0.7881 & 0.1213 & 0.7837 & 0.00557 \\
0.2227 & 0.6154 & 0.2210 & 0.6106 & 0.00777
\end{tabular}

$\begin{array}{lllll}0.2202 & 0.1688 & 0.2176 & 0.1668 & 0.01163 \\ 0.6942 & 0.1583 & 0.6860 & 0.1564 & 0.01190 \\ 0.1250 & 0.3082 & 0.1236 & 0.3049 & 0.01085 \\ 0.4273 & 0.1628 & 0.4222 & 0.1609 & 0.01188 \\ 0.1025 & 0.5557 & 0.1016 & 0.5510 & 0.00848 \\ 0.1059 & 0.6309 & 0.1051 & 0.6261 & 0.00754\end{array}$

${ }^{\mathrm{a}} x_{2}{ }^{(\mathrm{s})}$ : initial mole fraction of component 2 in the ternary solvent mixture, $x_{4}{ }^{(\mathrm{s})}$ : initial mole fraction of component 4 in the ternary solvent mixture; $x_{1}$ : mole fraction solubility of the solute; $x_{2}$ : mole fraction of component 2 in the quaternary solution; $x_{4}$ : mole fraction of component 4 in the quaternary solution.

\section{Method/Apparatus/Procedure:}

Constant-temperature bath, calorimetric thermometer, and an ultraviolet/ visible spectrophotometer.

Ternary solvent mixtures were prepared by mass. Excess solute and solvent were placed in amber glass bottles and allowed to equilibrate for several days at constant temperature. Attainment of equilibrium was verified by several repetitive measurements and by approaching equilibrium from supersaturation. Aliquots of saturated solutions were transferred through a coarse filter into volumetric flasks, weighed and diluted with methanol. Molar concentrations were determined by spectrophotometric measurements at $372 \mathrm{~nm}$.

\section{Source and Purity of Chemicals:}

(1) $98 \%$, Aldrich Chemical Company, Milwaukee, WI, USA, recrystallized three times from anhydrous methanol.

(2) $99.9 \%$, HPLC grade, Aldrich Chemical Company.

(3) $99 \%$, HPLC grade, Aldrich Chemical Company.

(4) $99+\%$, anhydrous, Aldrich Chemical Company.

Components 2-4 were stored over molecular sieves and distilled shortly before use.

Estimated Error:

Temperature: $\pm 0.1 \mathrm{~K}$.

$x_{2}{ }^{(\mathrm{s})}: \pm 0.0001$.

$x_{4}{ }^{(\mathrm{s})}: \pm 0.0001$.

$x_{1}: \pm 1.5 \%$ (relative error).

\begin{tabular}{|c|c|}
\hline $\begin{array}{l}\text { Components: } \\
\text { (1) Pyrene; } \mathrm{C}_{16} \mathrm{H}_{10} ;[129-00-0] \\
\text { (2) Cyclohexane; } \mathrm{C}_{6} \mathrm{H}_{12} ;[110-82-7] \\
\text { (3) Heptane; } \mathrm{C}_{7} \mathrm{H}_{16} ;[142-82-5] \\
\text { (4) 1-Butanol; } \mathrm{C}_{4} \mathrm{H}_{10} \mathrm{O} ;[71-36-3]\end{array}$ & $\begin{array}{l}\text { Original Measurements: } \\
{ }^{31} \text { E. M. Debase and W. E. Acree, } \\
\text { Jr., J. Chem. Eng. Data 46, } 1297 \\
\text { (2001). }\end{array}$ \\
\hline $\begin{array}{l}\text { Variables: } \\
T / \mathrm{K}=299.15 \text {; Solvent Composition }\end{array}$ & $\begin{array}{l}\text { Prepared by: } \\
\text { W. E. Acree, Jr. }\end{array}$ \\
\hline
\end{tabular}


Experimental Values

Solubility of pyrene in cyclohexane + heptane +1 -butanol mixtures ${ }^{\mathrm{a}}$

\begin{tabular}{|c|c|c|c|c|}
\hline$x_{2}{ }^{(\mathrm{s})}$ & $x_{4}{ }^{(\mathrm{s})}$ & $x_{2}$ & $x_{4}$ & $x_{1}$ \\
\hline 0.0000 & 0.0000 & 0.0000 & 0.0000 & 0.01102 \\
\hline 1.0000 & 0.0000 & 0.9890 & 0.0000 & 0.01100 \\
\hline 0.0000 & 1.0000 & 0.0000 & 0.9938 & 0.00622 \\
\hline 0.3345 & 0.4068 & 0.3306 & 0.4021 & 0.01159 \\
\hline 0.7051 & 0.1855 & 0.6964 & 0.1832 & 0.01233 \\
\hline 0.4535 & 0.2629 & 0.4480 & 0.2597 & 0.01219 \\
\hline 0.5010 & 0.2919 & 0.4949 & 0.2884 & 0.01215 \\
\hline 0.0988 & 0.7669 & 0.0980 & 0.7604 & 0.00846 \\
\hline 0.1875 & 0.7432 & 0.1859 & 0.7367 & 0.00868 \\
\hline 0.6853 & 0.2360 & 0.6770 & 0.2331 & 0.01218 \\
\hline 0.5520 & 0.1381 & 0.5451 & 0.1364 & 0.01243 \\
\hline 0.4787 & 0.4539 & 0.4734 & 0.4489 & 0.01110 \\
\hline 0.3759 & 0.5528 & 0.3720 & 0.5471 & 0.01029 \\
\hline 0.1775 & 0.2166 & 0.1754 & 0.2140 & 0.01204 \\
\hline 0.1391 & 0.7568 & 0.1379 & 0.7503 & 0.00861 \\
\hline 0.2416 & 0.5759 & 0.2391 & 0.5699 & 0.01035 \\
\hline 0.2330 & 0.1468 & 0.2302 & 0.1450 & 0.01218 \\
\hline 0.7131 & 0.1318 & 0.7043 & 0.1302 & 0.01229 \\
\hline 0.1223 & 0.2776 & 0.1208 & 0.2743 & 0.01194 \\
\hline 0.4468 & 0.1432 & 0.4413 & 0.1414 & 0.01239 \\
\hline 0.1151 & 0.5024 & 0.1139 & 0.4969 & 0.01085 \\
\hline 0.1094 & 0.5965 & 0.1083 & 0.5905 & 0.01007 \\
\hline
\end{tabular}

${ }^{\mathrm{a}} x_{2}{ }^{(\mathrm{s})}$ : initial mole fraction of component 2 in the ternary solvent mixture, $x_{4}{ }^{(\mathrm{s})}$ : initial mole fraction of component 4 in the ternary solvent mixture; $x_{1}$ : mole fraction solubility of the solute; $x_{2}$ : mole fraction of component 2 in the quaternary solution; $x_{4}$ : mole fraction of component 4 in the quaternary solution.

\section{Auxiliary Information}

\section{Method/Apparatus/Procedure:}

Constant-temperature bath, calorimetric thermometer, and an ultraviolet/ visible spectrophotometer.

Ternary solvent mixtures were prepared by mass. Excess solute and solvent were placed in amber glass bottles and allowed to equilibrate for several days at constant temperature. Attainment of equilibrium was verified by several repetitive measurements and by approaching equilibrium from supersaturation. Aliquots of saturated solutions were transferred through a coarse filter into volumetric flasks, weighed and diluted with methanol. Molar concentrations were determined by spectrophotometric measurements at $372 \mathrm{~nm}$.

\section{Source and Purity of Chemicals:}

(1) $98 \%$, Aldrich Chemical Company, Milwaukee, WI, USA, recrystallized three times from anhydrous methanol.

(2) $99.9 \%$, HPLC grade, Aldrich Chemical Company.

(3) $99 \%$, HPLC grade, Aldrich Chemical Company.

(4) $99+\%$, anhydrous, Aldrich Chemical Company.

Components $2-4$ were stored over molecular sieves and distilled shortly before use.

Estimated Error:

Temperature: $\pm 0.1 \mathrm{~K}$.

$x_{2}{ }^{(\mathrm{s})}: \pm 0.0001$.

$x_{4}{ }^{(\mathrm{s})}: \pm 0.0001$

$x_{1}: \pm 1.5 \%$ (relative error).

\begin{tabular}{|c|c|}
\hline $\begin{array}{l}\text { Components: } \\
\text { (1) Pyrene; } \mathrm{C}_{16} \mathrm{H}_{10} ;[129-00-0] \\
\text { (2) Cyclohexane; } \mathrm{C}_{6} \mathrm{H}_{12} ;[110-82-7] \\
\text { (3) Heptane; } \mathrm{C}_{7} \mathrm{H}_{16} ;[142-82-5] \\
\text { (4) 2-Butanol; } \mathrm{C}_{4} \mathrm{H}_{10} \mathrm{O} ;[78-92-2]\end{array}$ & $\begin{array}{l}\text { Original Measurements: } \\
{ }^{31} \text { E. M. Debase and W. E. Acree, } \\
\text { Jr., J. Chem. Eng. Data 46, } 1297 \\
\text { (2001). }\end{array}$ \\
\hline $\begin{array}{l}\text { Variables: } \\
T / \mathrm{K}=299.15 ; \text { Solvent Composition }\end{array}$ & $\begin{array}{l}\text { Prepared by: } \\
\text { W. E. Acree, Jr. }\end{array}$ \\
\hline
\end{tabular}

Experimental Values

Solubility of pyrene in cyclohexane + heptane +2 -butanol mixtures ${ }^{\mathrm{a}}$

\begin{tabular}{|c|c|c|c|c|}
\hline$x_{2}{ }^{(\mathrm{s})}$ & $x_{4}{ }^{(\mathrm{s})}$ & $x_{2}$ & $x_{4}$ & $x_{1}$ \\
\hline 0.0000 & 0.0000 & 0.0000 & 0.0000 & 0.01102 \\
\hline 1.0000 & 0.0000 & 0.9890 & 0.0000 & 0.01100 \\
\hline 0.0000 & 1.0000 & 0.0000 & 0.9956 & 0.00439 \\
\hline 0.3407 & 0.4054 & 0.3369 & 0.4008 & 0.01124 \\
\hline 0.7051 & 0.1862 & 0.6966 & 0.1840 & 0.01208 \\
\hline 0.2654 & 0.3354 & 0.2623 & 0.3315 & 0.01159 \\
\hline 0.5033 & 0.3041 & 0.4975 & 0.3006 & 0.01158 \\
\hline 0.0971 & 0.7602 & 0.0964 & 0.7546 & 0.00742 \\
\hline 0.1804 & 0.7513 & 0.1803 & 0.7503 & 0.00762 \\
\hline 0.6902 & 0.2344 & 0.6821 & 0.2316 & 0.01174 \\
\hline 0.5376 & 0.1329 & 0.5310 & 0.1313 & 0.01222 \\
\hline 0.4671 & 0.4472 & 0.4621 & 0.4424 & 0.01065 \\
\hline 0.3813 & 0.5460 & 0.3776 & 0.5407 & 0.00962 \\
\hline 0.1304 & 0.2277 & 0.1289 & 0.2250 & 0.01170 \\
\hline 0.1415 & 0.7576 & 0.1404 & 0.7519 & 0.00748 \\
\hline 0.2526 & 0.5699 & 0.2504 & 0.5650 & 0.00860 \\
\hline 0.2403 & 0.1508 & 0.2374 & 0.1490 & 0.01209 \\
\hline 0.7118 & 0.1357 & 0.7031 & 0.1340 & 0.01223 \\
\hline 0.1286 & 0.2737 & 0.1271 & 0.2705 & 0.01168 \\
\hline 0.4416 & 0.1532 & 0.4362 & 0.1513 & 0.01220 \\
\hline 0.1125 & 0.5036 & 0.1114 & 0.4985 & 0.01022 \\
\hline 0.1077 & 0.5894 & 0.1067 & 0.5839 & 0.00936 \\
\hline
\end{tabular}

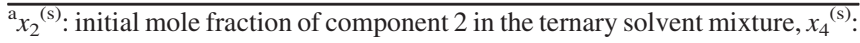
initial mole fraction of component 4 in the ternary solvent mixture; $x_{1}$ : mole fraction solubility of the solute; $x_{2}$ : mole fraction of component 2 in the quaternary solution; $x_{4}$ : mole fraction of component 4 in the quaternary solution.

\section{Auxiliary Information}

\section{Method/Apparatus/Procedure:}

Constant-temperature bath, calorimetric thermometer, and an ultraviolet/ visible spectrophotometer.

Ternary solvent mixtures were prepared by mass. Excess solute and solvent were placed in amber glass bottles and allowed to equilibrate for several days at constant temperature. Attainment of equilibrium was verified by several repetitive measurements and by approaching equilibrium from supersaturation. Aliquots of saturated solutions were transferred through a coarse filter into volumetric flasks, weighed and diluted with methanol. Molar concentrations were determined by spectrophotometric measurements at $372 \mathrm{~nm}$.

Source and Purity of Chemicals:

(1) $98 \%$, Aldrich Chemical Company, Milwaukee, WI, USA, recrystallized three times from anhydrous methanol.

(2) $99.9 \%$, HPLC grade, Aldrich Chemical Company.

(3) $99 \%$, HPLC grade, Aldrich Chemical Company.

(4) $99+\%$, anhydrous, Aldrich Chemical Company.

Components $2-4$ were stored over molecular sieves and distilled shortly before use. 
Estimated Error:

Temperature: $\pm 0.1 \mathrm{~K}$

$x_{2}{ }^{(\mathrm{s})}: \pm 0.0001$

$x_{4}{ }^{(\mathrm{s})}: \pm 0.0001$.

$x_{1}: \pm 1.5 \%$ (relative error)

\begin{tabular}{|c|c|}
\hline $\begin{array}{l}\text { Components: } \\
\text { (1) Pyrene; } \mathrm{C}_{16} \mathrm{H}_{10} ;[129-00-0] \\
\text { (2) Cyclohexane; } \mathrm{C}_{6} \mathrm{H}_{12} ;[110-82-7] \\
\text { (3) Heptane; } \mathrm{C}_{7} \mathrm{H}_{16} ;[142-82-5] \\
\text { (4) 2-Methyl-1-propanol; } \mathrm{C}_{4} \mathrm{H}_{10} \mathrm{O} \text {; } \\
\text { [78-83-1] }\end{array}$ & $\begin{array}{l}\text { Original Measurements: } \\
{ }^{31} \text { E. M. Debase and W. E. Acree, } \\
\text { Jr., J. Chem. Eng. Data 46, } 1297 \\
(2001) \text {. }\end{array}$ \\
\hline $\begin{array}{l}\text { Variables: } \\
T / \mathrm{K}=299.15 \text {; Solvent Composition }\end{array}$ & $\begin{array}{l}\text { Prepared by: } \\
\text { W. E. Acree, Jr. }\end{array}$ \\
\hline
\end{tabular}

Experimental Values

Solubility of pyrene in cyclohexane + heptane +2 -methyl-1-propanol mixtures $^{\mathrm{a}}$

\begin{tabular}{lcccc}
\hline \hline$x_{2}{ }^{(\mathrm{s})}$ & $x_{4}{ }^{(\mathrm{s})}$ & $x_{2}$ & $x_{4}$ & $x_{1}$ \\
\hline 0.0000 & 0.0000 & 0.0000 & 0.0000 & 0.01102 \\
1.0000 & 0.0000 & 0.9890 & 0.0000 & 0.01100 \\
0.0000 & 1.0000 & 0.0000 & 0.9967 & 0.00326 \\
0.3471 & 0.3977 & 0.3436 & 0.3937 & 0.01009 \\
0.6971 & 0.1900 & 0.6891 & 0.1878 & 0.01141 \\
0.2734 & 0.3231 & 0.2705 & 0.3196 & 0.01074 \\
0.5178 & 0.3002 & 0.5122 & 0.2970 & 0.01076 \\
0.0951 & 0.7610 & 0.0588 & 0.7565 & 0.00586 \\
0.1837 & 0.7559 & 0.1827 & 0.7516 & 0.00568 \\
0.6945 & 0.2339 & 0.6868 & 0.2313 & 0.01103 \\
0.5481 & 0.1347 & 0.5416 & 0.1331 & 0.01191 \\
0.4675 & 0.4568 & 0.4632 & 0.4526 & 0.00914 \\
0.3788 & 0.5524 & 0.3757 & 0.5479 & 0.00818 \\
0.1780 & 0.2139 & 0.1760 & 0.2115 & 0.01135 \\
0.1436 & 0.7555 & 0.1428 & 0.7511 & 0.00587 \\
0.2460 & 0.5735 & 0.2440 & 0.5689 & 0.00800 \\
0.2322 & 0.1465 & 0.2295 & 0.1448 & 0.01183 \\
0.7147 & 0.1345 & 0.7063 & 0.1329 & 0.01171 \\
0.1219 & 0.2834 & 0.1206 & 0.2803 & 0.01098 \\
0.4479 & 0.1369 & 0.4426 & 0.1353 & 0.01181 \\
0.1078 & 0.5026 & 0.1068 & 0.4981 & 0.00905 \\
0.1033 & 0.5980 & 0.1025 & 0.5933 & 0.00787 \\
\hline$x_{2} \mathrm{~s}:$ & 0.969
\end{tabular}

${ }^{\mathrm{a}} x_{2}{ }^{(\mathrm{s})}$ : initial mole fraction of component 2 in the ternary solvent mixture, $x_{4}{ }^{(\mathrm{s})}$ : initial mole fraction of component 4 in the ternary solvent mixture; $x_{1}$ : mole fraction solubility of the solute; $x_{2}$ : mole fraction of component 2 in the quaternary solution; $x_{4}$ : mole fraction of component 4 in the quaternary solution.

\section{Auxiliary Information}

\section{Method/Apparatus/Procedure:}

Constant-temperature bath, calorimetric thermometer, and an ultraviolet/ visible spectrophotometer.

Ternary solvent mixtures were prepared by mass. Excess solute and solvent were placed in amber glass bottles and allowed to equilibrate for several days at constant temperature. Attainment of equilibrium was verified by several repetitive measurements and by approaching equilibrium from supersaturation. Aliquots of saturated solutions were transferred through a coarse filter into volumetric flasks, weighed and diluted with methanol. Molar concentrations were determined by spectrophotometric measurements at $372 \mathrm{~nm}$.
Source and Purity of Chemicals:

(1) $98 \%$, Aldrich Chemical Company, Milwaukee, WI, USA, recrystallized three times from anhydrous methanol.

(2) $99.9 \%$, HPLC grade, Aldrich Chemical Company.

(3) $99 \%$, HPLC grade, Aldrich Chemical Company.

(4) $99+\%$, anhydrous, Aldrich Chemical Company.

Components 2-4 were stored over molecular sieves and distilled shortly before use.

Estimated Error:

Temperature: $\pm 0.1 \mathrm{~K}$.

$x_{2}{ }^{(s)}: \pm 0.0001$.

$x_{4}{ }^{(\mathrm{s})}: \pm 0.0001$.

$x_{1}: \pm 1.5 \%$ (relative error).

\section{Solubility of Pyrene in Ternary Alkane + Alcohol + Alcohol Solvent Mixtures}

\subsection{Critical evaluation of experimental solubility data}

Fishback et al. ${ }^{29}$ and Debase and Acree ${ }^{30,32}$ determined the solubility of pyrene in ternary heptane + propanol + butanol, cyclohexane + propanol + butanol, and 2,2,4-trimethylpentane + propanol + butanol solvent mixtures at $299.15 \mathrm{~K}$. There has only been a single experimental determination for the solubility of pyrene in each of the ternary solvent systems studied. In the absence of replicate independent measurements, the experimental values were evaluated by curve-fitting the measured binary solvent solubility data to Eq. (1), and then using the ternary solvent version of the basic model, Eq. (2), to estimate the mole fraction solubility of pyrene dissolved in ternary alkane + alcohol + alcohol solvent mixtures.

The calculated $S_{23, i}$ parameters for all of the contributing binary alkane + alcohol and alcohol + alcohol solvent systems studied are summarized in Table 2, along with the average absolute deviation between the experimental and back-calculated mole fraction solubilities. The predictive ability of Eq. (2) is given in Table 12 for the 12 ternary alkane + alcohol + alcohol solvent systems. Examination of the numerical entries in the last column of Table 12 reveals that Eq. (2) does provide fairly accurate mathematical predictions for how the measured

TABLE 12. Summarized comparison between experimental solubilities of pyrene in ternary alkane + alcohol + alcohol solvent mixtures and predicted values based on Eq. (2)

\begin{tabular}{lc}
\hline \hline Ternary solvent mixture & $\%$ Dev. \\
\hline Heptane + 1-propanol + 1-butanol & 1.74 \\
Heptane + 2-propanol + 1-butanol & 1.45 \\
Heptane + 1-propanol + 2-butanol & 1.70 \\
Heptane + 2-propanol + 2-butanol & 1.36 \\
Cyclohexane + 1-propanol + 1-butanol & 1.43 \\
Cyclohexane + 2-propanol + 1-butanol & 1.06 \\
Cyclohexane + 1-propanol + 2-butanol & 0.96 \\
Cyclohexane + 2-propanol + 2-butanol & 1.44 \\
2,2,4-Trimethylpentane + 1-propanol + 1-butanol & 1.66 \\
2,2,4-Trimethylpentane + 2-propanol + 1-butanol & 1.42 \\
2,2,4-Trimethylpentane + 1-propanol + 2-butanol & 1.69 \\
2,2,4-Trimethylpentane + 2-propanol + 2-butanol & 1.62 \\
\hline${ }^{\mathrm{a}}$ Dev (\%) $=(100 / N) \Sigma\left|\left[x_{1}{ }^{\text {exp }}-x_{1}{ }^{\text {cal }}\right] / x_{1}{ }^{\exp }\right|$. &
\end{tabular}


mole fraction solubilities vary with ternary solvent composition. Average percent deviations range from $1.0 \%$ to $1.7 \%$, suggesting that there are no obvious outliers in an individual ternary solvent data set. None of the experimental data points was flagged as an outlier.

The experimental pyrene solubility data for ternary heptane + propanol + butanol, cyclohexane + propanol + butanol, and 2,2,4-trimethylpentane + propanol + butanol solvent mixtures are tabulated in Secs. 12.2-12.4.

\subsection{Pyrene solubility data in ternary heptane + alcohol + alcohol solvent mixtures}

\begin{tabular}{ll}
\hline \hline Components: & Original Measurements: \\
(1) Pyrene; $\mathrm{C}_{16} \mathrm{H}_{10} ;[129-00-0]$ & ${ }^{29} \mathrm{~S}$. Fishback, S. Duenas, N. \\
(2) Heptane; $\mathrm{C}_{7} \mathrm{H}_{16} ;[142-82-5]$ & Kuehn, J. Pacheco, and W. E. \\
(3) 1-Propanol; $\mathrm{C}_{3} \mathrm{H}_{8} \mathrm{O} ;[71-23-8]$ & Acree, Jr., J. Chem. Eng. Data 47, \\
(4) 1-Butanol; $\mathrm{C}_{4} \mathrm{H}_{10} \mathrm{O} ;[71-36-3]$ & $62(2002)$. \\
\hline Variables: & Prepared by: \\
$T / \mathrm{K}=299.15 ;$ Solvent Composition & W. E. Acree, Jr. \\
\hline
\end{tabular}

Experimental Values

Solubility of pyrene in heptane +1 -propanol +1 -butanol mixtures ${ }^{\mathrm{a}}$

\begin{tabular}{|c|c|c|c|c|}
\hline$x_{3}{ }^{(\mathrm{s})}$ & $x_{4}{ }^{(\mathrm{s})}$ & $x_{3}$ & $x_{4}$ & $x_{1}$ \\
\hline 0.0000 & 0.0000 & 0.0000 & 0.0000 & 0.01102 \\
\hline 1.0000 & 0.0000 & 0.9957 & 0.0000 & 0.00426 \\
\hline 0.0000 & 1.0000 & 0.0000 & 0.9938 & 0.00622 \\
\hline 0.4268 & 0.3473 & 0.4235 & 0.3446 & 0.00784 \\
\hline 0.1904 & 0.7216 & 0.1891 & 0.7166 & 0.00689 \\
\hline 0.3478 & 0.2863 & 0.3446 & 0.2837 & 0.00916 \\
\hline 0.3137 & 0.5261 & 0.3114 & 0.5222 & 0.00734 \\
\hline 0.7862 & 0.0923 & 0.7814 & 0.0917 & 0.00605 \\
\hline 0.7548 & 0.1880 & 0.7506 & 0.1869 & 0.00560 \\
\hline 0.2449 & 0.6912 & 0.2433 & 0.6867 & 0.00651 \\
\hline 0.1503 & 0.5605 & 0.1490 & 0.5555 & 0.00891 \\
\hline 0.4578 & 0.4763 & 0.4550 & 0.4734 & 0.00608 \\
\hline 0.5646 & 0.3767 & 0.5613 & 0.3745 & 0.00582 \\
\hline 0.2405 & 0.2000 & 0.2379 & 0.1978 & 0.01099 \\
\hline 0.7624 & 0.1528 & 0.7580 & 0.1519 & 0.00575 \\
\hline 0.5900 & 0.2515 & 0.5860 & 0.2498 & 0.00677 \\
\hline 0.1687 & 0.2741 & 0.1668 & 0.2711 & 0.01109 \\
\hline 0.1371 & 0.7247 & 0.1361 & 0.7193 & 0.00749 \\
\hline 0.3088 & 0.1357 & 0.3055 & 0.1342 & 0.01073 \\
\hline 0.1610 & 0.4716 & 0.1593 & 0.4671 & 0.00958 \\
\hline 0.5346 & 0.1263 & 0.5300 & 0.1252 & 0.00860 \\
\hline 0.6266 & 0.1086 & 0.6216 & 0.1077 & 0.00798 \\
\hline \multicolumn{5}{|c|}{$\begin{array}{l}{ }^{a} x_{3}{ }^{(\mathrm{s})} \text { : initial mole fraction of component } 3 \text { in the ternary solvent mixture, } x_{4}{ }^{(\mathrm{s}} \\
\text { initial mole fraction of component } 4 \text { in the ternary solvent mixture; } x_{1}: \text { mol } \\
\text { fraction solubility of the solute; } x_{3} \text { : mole fraction of component } 3 \text { in the } \\
\text { quaternary solution; } x_{4} \text { : mole fraction of component } 4 \text { in the quaternary } \\
\text { solution. }\end{array}$} \\
\hline
\end{tabular}

\section{Auxiliary Information}

\section{Method/Apparatus/Procedure:}

Constant-temperature bath, calorimetric thermometer, and an ultraviolet/ visible spectrophotometer.
Ternary solvent mixtures were prepared by mass. Excess solute and solvent were placed in amber glass bottles and allowed to equilibrate for several days at constant temperature. Attainment of equilibrium was verified by several repetitive measurements and by approaching equilibrium from supersaturation. Aliquots of saturated solutions were transferred through a coarse filter into volumetric flasks, weighed and diluted with methanol. Molar concentrations were determined by spectrophotometric measurements at $372 \mathrm{~nm}$.

\section{Source and Purity of Chemicals:}

(1) $98 \%$, Aldrich Chemical Company, Milwaukee, WI, USA, recrystallized three times from anhydrous methanol.

(2) $99 \%$, HPLC grade, Aldrich Chemical Company.

(3) $99+\%$, anhydrous, Aldrich Chemical Company.

(4) $99+\%$, anhydrous, Aldrich Chemical Company.

Components 2-4 were stored over molecular sieves and distilled shortly before use.

\section{Estimated Error:}

Temperature: $\pm 0.1 \mathrm{~K}$.

$x_{3}{ }^{(s)}: \pm 0.0001$.

$x_{4}{ }^{(\mathrm{s})}: \pm 0.0001$.

$x_{1}: \pm 1.5 \%$ (relative error).

\begin{tabular}{ll}
\hline \hline Components: & Original Measurements: \\
(1) Pyrene; $\mathrm{C}_{16} \mathrm{H}_{10} ;[129-00-0]$ & ${ }^{29}$ S. Fishback, S. Duenas, N. \\
(2) Heptane; $\mathrm{C}_{7} \mathrm{H}_{16} ;[142-82-5]$ & Kuehn, J. Pacheco, and W. E. \\
(3) 2-Propanol; $\mathrm{C}_{3} \mathrm{H}_{8} \mathrm{O} ;[67-63-0]$ & Acree, Jr., J. Chem. Eng. Data 47, \\
(4) 1-Butanol; $\mathrm{C}_{4} \mathrm{H}_{10} \mathrm{O} ;[71-36-3]$ & $62(2002)$. \\
\hline Variables: & Prepared by: \\
$T / \mathrm{K}=299.15 ;$ Solvent Composition & W. E. Acree, Jr. \\
\hline
\end{tabular}

Experimental Values

Solubility of pyrene in heptane +2 -propanol +1 -butanol mixtures ${ }^{\mathrm{a}}$

\begin{tabular}{lcccc}
\hline \hline$x_{3}{ }^{(\mathrm{s})}$ & $x_{4}{ }^{(\mathrm{s})}$ & $x_{3}$ & $x_{4}$ & $x_{1}$ \\
\hline 0.0000 & 0.0000 & 0.0000 & 0.0000 & 0.01102 \\
1.0000 & 0.0000 & 0.9971 & 0.0000 & 0.00290 \\
0.0000 & 1.0000 & 0.0000 & 0.9938 & 0.00622 \\
0.4260 & 0.3485 & 0.4230 & 0.3460 & 0.00708 \\
0.1891 & 0.7151 & 0.1878 & 0.7104 & 0.00663 \\
0.3457 & 0.2954 & 0.3428 & 0.2929 & 0.00847 \\
0.3120 & 0.5326 & 0.3099 & 0.5290 & 0.00676 \\
0.7617 & 0.0855 & 0.7578 & 0.0851 & 0.00507 \\
0.7621 & 0.1806 & 0.7589 & 0.1798 & 0.00420 \\
0.2429 & 0.6918 & 0.2414 & 0.6875 & 0.00623 \\
0.1416 & 0.5685 & 0.1404 & 0.5636 & 0.00866 \\
0.4558 & 0.4820 & 0.4534 & 0.4794 & 0.00536 \\
0.5569 & 0.3776 & 0.5541 & 0.3757 & 0.00498 \\
0.2383 & 0.1934 & 0.2368 & 0.1914 & 0.01053 \\
0.7364 & 0.1824 & 0.7331 & 0.1816 & 0.00453 \\
0.5874 & 0.2533 & 0.5840 & 0.2518 & 0.00573 \\
0.1624 & 0.2618 & 0.1607 & 0.2591 & 0.01038 \\
0.1236 & 0.7389 & 0.1227 & 0.7335 & 0.00731 \\
0.3130 & 0.1334 & 0.3099 & 0.1321 & 0.00991 \\
0.1500 & 0.4773 & 0.1486 & 0.4728 & 0.00942 \\
0.5294 & 0.1233 & 0.5253 & 0.1223 & 0.00782 \\
0.6180 & 0.1175 & 0.6138 & 0.1167 & 0.00672 \\
\hline${ }_{3}{ }_{3}^{(\mathrm{s})}:$ initial mole fraction of component 3 in the ternary solvent mixture, $x_{4}^{(\mathrm{s})}$ : & initial mole fraction of component 4 in the ternary solvent mixture; $x_{1}:$ mole \\
fraction solubility of the solute; $x_{3}:$ mole fraction of component 3 in the \\
quaternary solution; $x_{4}:$ mole fraction of component 4 in the quaternary \\
solution. & & & & \\
& & & &
\end{tabular}




\section{Auxiliary Information}

\section{Method/Apparatus/Procedure:}

Constant-temperature bath, calorimetric thermometer, and an ultraviolet/ visible spectrophotometer.

Ternary solvent mixtures were prepared by mass. Excess solute and solvent were placed in amber glass bottles and allowed to equilibrate for several days at constant temperature. Attainment of equilibrium was verified by several repetitive measurements and by approaching equilibrium from supersaturation. Aliquots of saturated solutions were transferred through a coarse filter into volumetric flasks, weighed and diluted with methanol. Molar concentrations were determined by spectrophotometric measurements at $372 \mathrm{~nm}$.

\section{Source and Purity of Chemicals:}

(1) $98 \%$, Aldrich Chemical Company, Milwaukee, WI, USA, recrystallized three times from anhydrous methanol.

(2) $99 \%$, HPLC grade, Aldrich Chemical Company.

(3) $99+\%$, anhydrous, Aldrich Chemical Company.

(4) $99+\%$, anhydrous, Aldrich Chemical Company.

Components $2-4$ were stored over molecular sieves and distilled shortly before use.

\section{Estimated Error:}

Temperature: $\pm 0.1 \mathrm{~K}$

$x_{3}{ }^{(\mathrm{s})}: \pm 0.0001$.

$x_{4}{ }^{(\mathrm{s})}: \pm 0.0001$.

$x_{1}: \pm 1.5 \%$ (relative error).

\section{Components:}

(1) Pyrene; $\mathrm{C}_{16} \mathrm{H}_{10} ;[129-00-0]$

(2) Heptane; $\mathrm{C}_{7} \mathrm{H}_{16} ;[142-82-5]$

(3) 1-Propanol; $\mathrm{C}_{3} \mathrm{H}_{8} \mathrm{O}$; [71-23-8]

(4) 2-Butanol; $\mathrm{C}_{4} \mathrm{H}_{10} \mathrm{O} ;[$ [78-92-2]

\section{Original Measurements:}

${ }^{29}$ S. Fishback, S. Duenas, N. Kuehn, J. Pacheco, and W. E. Acree, Jr., J. Chem. Eng. Data 47, $62(2002)$

\begin{tabular}{ll}
\hline Variables: & Prepared by: \\
$T / \mathrm{K}=299.15 ;$ Solvent Composition & W. E. Acree, Jr. \\
\hline
\end{tabular}

Experimental Values

Solubility of pyrene in heptane +1 -propanol +2 -butanol mixtures ${ }^{\mathrm{a}}$

\begin{tabular}{lcccc}
\hline \hline$x_{3}{ }^{(\mathrm{s})}$ & $x_{4}{ }^{(\mathrm{s})}$ & $x_{3}$ & $x_{4}$ & $x_{1}$ \\
\hline 0.0000 & 0.0000 & 0.0000 & 0.0000 & 0.01102 \\
1.0000 & 0.0000 & 0.9957 & 0.0000 & 0.00426 \\
0.0000 & 1.0000 & 0.0000 & 0.9956 & 0.00439 \\
0.4121 & 0.3645 & 0.4092 & 0.3619 & 0.00701 \\
0.1800 & 0.7236 & 0.1790 & 0.7195 & 0.00564 \\
0.3364 & 0.2948 & 0.3335 & 0.2922 & 0.00871 \\
0.3126 & 0.5283 & 0.3106 & 0.5249 & 0.00638 \\
0.7874 & 0.0952 & 0.7828 & 0.0946 & 0.00581 \\
0.7481 & 0.1966 & 0.7443 & 0.1956 & 0.00504 \\
0.2276 & 0.7153 & 0.2264 & 0.7116 & 0.00520 \\
0.1313 & 0.5819 & 0.1302 & 0.5772 & 0.00813 \\
0.4578 & 0.4799 & 0.4554 & 0.4774 & 0.00517 \\
0.5656 & 0.3757 & 0.5627 & 0.3738 & 0.00507 \\
0.2324 & 0.1840 & 0.2299 & 0.1820 & 0.01071 \\
0.7708 & 0.1359 & 0.7667 & 0.1352 & 0.00535
\end{tabular}

$\begin{array}{lllll}0.6009 & 0.2438 & 0.5972 & 0.2423 & 0.00622 \\ 0.1114 & 0.3023 & 0.1102 & 0.2990 & 0.01086 \\ 0.1173 & 0.7440 & 0.1166 & 0.7394 & 0.00629 \\ 0.3165 & 0.1362 & 0.3132 & 0.1348 & 0.01033 \\ 0.1632 & 0.4591 & 0.1618 & 0.4550 & 0.00885 \\ 0.5306 & 0.1133 & 0.5261 & 0.1123 & 0.00853 \\ 0.6259 & 0.1045 & 0.6212 & 0.1037 & 0.00752\end{array}$

${ }^{\mathrm{a}}{ }_{x_{3}}{ }^{(\mathrm{s})}$ : initial mole fraction of component 3 in the ternary solvent mixture, $x_{4}{ }^{(\mathrm{s})}$ : initial mole fraction of component 4 in the ternary solvent mixture; $x_{1}$ : mole fraction solubility of the solute; $x_{3}$ : mole fraction of component 3 in the quaternary solution; $x_{4}$ : mole fraction of component 4 in the quaternary solution.

\section{Auxiliary Information}

\section{Method/Apparatus/Procedure:}

Constant-temperature bath, calorimetric thermometer, and an ultraviolet/ visible spectrophotometer.

Ternary solvent mixtures were prepared by mass. Excess solute and solvent were placed in amber glass bottles and allowed to equilibrate for several days at constant temperature. Attainment of equilibrium was verified by several repetitive measurements and by approaching equilibrium from supersaturation. Aliquots of saturated solutions were transferred through a coarse filter into volumetric flasks, weighed and diluted with methanol. Molar concentrations were determined by spectrophotometric measurements at $372 \mathrm{~nm}$.

\section{Source and Purity of Chemicals:}

(1) $98 \%$, Aldrich Chemical Company, Milwaukee, WI, USA, recrystallized three times from anhydrous methanol.

(2) $99 \%$, HPLC grade, Aldrich Chemical Company.

(3) $99+\%$, anhydrous, Aldrich Chemical Company.

(4) $99+\%$, anhydrous, Aldrich Chemical Company.

Components $2-4$ were stored over molecular sieves and distilled shortly before use.

\section{Estimated Error:}

Temperature: $\pm 0.1 \mathrm{~K}$.

$x_{3}{ }^{(\mathrm{s})}: \pm 0.0001$.

$x_{4}{ }^{(\mathrm{s})}: \pm 0.0001$.

$x_{1}: \pm 1.5 \%$ (relative error).

\begin{tabular}{ll}
\hline \hline Components: & Original Measurements: \\
(1) Pyrene; $\mathrm{C}_{16} \mathrm{H}_{10} ;[129-00-0]$ & ${ }^{29} \mathrm{~S}$. Fishback, S. Duenas, N. \\
(2) Heptane; $\mathrm{C}_{7} \mathrm{H}_{16} ;[142-82-5]$ & Kuehn, J. Pacheco, and W. E. \\
(3) 2-Propanol; $\mathrm{C}_{3} \mathrm{H}_{8} \mathrm{O} ;[67-63-0]$ & Acree, Jr., J. Chem. Eng. Data 47, \\
(4) 2-Butanol; $\mathrm{C}_{4} \mathrm{H}_{10} \mathrm{O} ;[78-92-2]$ & $62(2002)$. \\
\hline Variables: & Prepared by: \\
$T / \mathrm{K}=299.15 ;$ Solvent Composition & W. E. Acree, Jr. \\
\hline
\end{tabular}


Experimental Values

Solubility of pyrene in heptane +2 -propanol +2 -butanol mixtures ${ }^{\mathrm{a}}$

\begin{tabular}{|c|c|c|c|c|}
\hline$x_{3}{ }^{(\mathrm{s})}$ & $x_{4}{ }^{(\mathrm{s})}$ & $x_{3}$ & $x_{4}$ & $x_{1}$ \\
\hline 0.0000 & 0.0000 & 0.0000 & 0.0000 & 0.01102 \\
\hline 1.0000 & 0.0000 & 0.9971 & 0.0000 & 0.00290 \\
\hline 0.0000 & 1.0000 & 0.0000 & 0.9956 & 0.00439 \\
\hline 0.4256 & 0.3557 & 0.4229 & 0.3534 & 0.00643 \\
\hline 0.1939 & 0.7079 & 0.1928 & 0.7040 & 0.00552 \\
\hline 0.3249 & 0.2931 & 0.3222 & 0.2906 & 0.00840 \\
\hline 0.3231 & 0.5142 & 0.3211 & 0.5111 & 0.00604 \\
\hline 0.7823 & 0.0998 & 0.7787 & 0.0993 & 0.00455 \\
\hline 0.7542 & 0.1869 & 0.7511 & 0.1861 & 0.00412 \\
\hline 0.2420 & 0.6862 & 0.2408 & 0.6827 & 0.00515 \\
\hline 0.1477 & 0.5632 & 0.1465 & 0.5588 & 0.00789 \\
\hline 0.4706 & 0.4670 & 0.4685 & 0.4649 & 0.00455 \\
\hline 0.5602 & 0.3759 & 0.5577 & 0.3742 & 0.00446 \\
\hline 0.2437 & 0.1969 & 0.2412 & 0.1949 & 0.01012 \\
\hline 0.7699 & 0.1406 & 0.7666 & 0.1400 & 0.00435 \\
\hline 0.5856 & 0.2544 & 0.5824 & 0.2530 & 0.00552 \\
\hline 0.1731 & 0.2669 & 0.1713 & 0.2642 & 0.01030 \\
\hline 0.1311 & 0.7358 & 0.1303 & 0.7313 & 0.00605 \\
\hline 0.3008 & 0.1362 & 0.2978 & 0.1348 & 0.01008 \\
\hline 0.1517 & 0.4706 & 0.1503 & 0.4664 & 0.00895 \\
\hline 0.5248 & 0.1234 & 0.5208 & 0.1225 & 0.00764 \\
\hline 0.6183 & 0.1150 & 0.6142 & 0.1142 & 0.00657 \\
\hline
\end{tabular}

${ }^{\mathrm{a}} x_{3}{ }^{(\mathrm{s})}$ : initial mole fraction of component 3 in the ternary solvent mixture, $x_{4}{ }^{(\mathrm{s})}$. initial mole fraction of component 4 in the ternary solvent mixture; $x_{1}$ : mole fraction solubility of the solute; $x_{3}$ : mole fraction of component 3 in the quaternary solution; $x_{4}$ : mole fraction of component 4 in the quaternary solution.

\section{Auxiliary Information}

Method/Apparatus/Procedure:

Constant-temperature bath, calorimetric thermometer, and an ultraviolet/ visible spectrophotometer.

Ternary solvent mixtures were prepared by mass. Excess solute and solvent were placed in amber glass bottles and allowed to equilibrate for several days at constant temperature. Attainment of equilibrium was verified by several repetitive measurements and by approaching equilibrium from supersaturation. Aliquots of saturated solutions were transferred through a coarse filter into volumetric flasks, weighed and diluted with methanol. Molar concentrations were determined by spectrophotometric measurements at $372 \mathrm{~nm}$.

Source and Purity of Chemicals:

(1) $98 \%$, Aldrich Chemical Company, Milwaukee, WI, USA, recrystallized three times from anhydrous methanol.

(2) $99 \%$, HPLC grade, Aldrich Chemical Company.

(3) $99+\%$, anhydrous, Aldrich Chemical Company.

(4) $99+\%$, anhydrous, Aldrich Chemical Company.

Components 2-4 were stored over molecular sieves and distilled shortly before use.

Estimated Error:

Temperature: $\pm 0.1 \mathrm{~K}$.

$x_{3}{ }^{(\mathrm{s})}: \pm 0.0001$.

$x_{4}{ }^{(\mathrm{s})}: \pm 0.0001$.

$x_{1}: \pm 1.5 \%$ (relative error).
12.3. Pyrene solubility data in ternary cyclohexane + alcohol + alcohol solvent mixtures

\begin{tabular}{ll}
\hline \hline Components: & Original Measurements: \\
(1) Pyrene; $\mathrm{C}_{16} \mathrm{H}_{10} ;[129-00-0]$ & ${ }^{32}$ E. M. Debase and W. E. Acree, \\
(2) Cyclohexane; $\mathrm{C}_{6} \mathrm{H}_{12} ;[110-82-7]$ & Jr., J. Chem. Eng. Data 46, 991 \\
(3) 1-Propanol; $\mathrm{C}_{3} \mathrm{H}_{8} \mathrm{O} ;[71-23-8]$ & $(2001)$. \\
(4) 1-Butanol; $\mathrm{C}_{4} \mathrm{H}_{10} \mathrm{O} ;[71-36-3]$ & \\
\hline Variables: & Prepared by: \\
$T / \mathrm{K}=299.15 ;$ Solvent Composition & W. E. Acree, Jr. \\
\hline
\end{tabular}

Experimental Values

Solubility of pyrene in cyclohexane +1 -propanol +1 -butanol mixtures ${ }^{\mathrm{a}}$

\begin{tabular}{lcccc}
\hline \hline$x_{3}{ }^{(\mathrm{s})}$ & $x_{4}{ }^{(\mathrm{s})}$ & $x_{3}$ & $x_{4}$ & $x_{1}$ \\
\hline 0.0000 & 0.0000 & 0.0000 & 0.0000 & 0.01100 \\
1.0000 & 0.0000 & 0.9957 & 0.0000 & 0.00426 \\
0.0000 & 1.0000 & 0.0000 & 0.9938 & 0.00622 \\
0.3996 & 0.3217 & 0.3963 & 0.3190 & 0.00826 \\
0.1828 & 0.6929 & 0.1815 & 0.6879 & 0.00718 \\
0.3156 & 0.2545 & 0.3126 & 0.2521 & 0.00960 \\
0.3003 & 0.4963 & 0.2980 & 0.4925 & 0.00762 \\
0.7506 & 0.0984 & 0.7460 & 0.0978 & 0.00617 \\
0.7400 & 0.1808 & 0.7360 & 0.1798 & 0.00533 \\
0.2374 & 0.6769 & 0.2358 & 0.6724 & 0.00665 \\
0.1367 & 0.5129 & 0.1354 & 0.5082 & 0.00921 \\
0.4579 & 0.4597 & 0.4551 & 0.4568 & 0.00622 \\
0.5612 & 0.3576 & 0.5579 & 0.3555 & 0.00595 \\
01954 & 0.1593 & 0.1952 & 0.1591 & 0.01177 \\
0.7515 & 0.1345 & 0.7471 & 0.1337 & 0.00587 \\
0.5642 & 0.2383 & 0.5602 & 0.2366 & 0.00701 \\
0.1414 & 0.2154 & 0.1398 & 0.2129 & 0.01159 \\
0.1262 & 0.7009 & 0.1252 & 0.6956 & 0.00756 \\
0.2726 & 0.1028 & 0.2696 & 0.1017 & 0.01118 \\
0.1407 & 0.4232 & 0.1393 & 0.4189 & 0.01015 \\
0.4867 & 0.1066 & 0.4822 & 0.1056 & 0.00925 \\
0.5812 & 0.1014 & 0.5765 & 0.1006 & 0.00817 \\
\hline$x_{3}{ }^{(\mathrm{s})}: 1 \mathrm{n}$ & 0.569 &
\end{tabular}

${ }^{\mathrm{a}} x_{3}{ }^{(\mathrm{s})}$ : initial mole fraction of component 3 in the ternary solvent mixture, $x_{4}{ }^{(\mathrm{s})}$ : initial mole fraction of component 4 in the ternary solvent mixture; $x_{1}$ : mole fraction solubility of the solute; $x_{3}$ : mole fraction of component 3 in the quaternary solution; $x_{4}$ : mole fraction of component 4 in the quaternary solution.

\section{Method/Apparatus/Procedure:}

Constant-temperature bath, calorimetric thermometer, and an ultraviolet/ visible spectrophotometer.

Ternary solvent mixtures were prepared by mass. Excess solute and solvent were placed in amber glass bottles and allowed to equilibrate for several days at constant temperature. Attainment of equilibrium was verified by several repetitive measurements and by approaching equilibrium from supersaturation. Aliquots of saturated solutions were transferred through a coarse filter into volumetric flasks, weighed and diluted with methanol. Molar concentrations were determined by spectrophotometric measurements at $372 \mathrm{~nm}$. 
Source and Purity of Chemicals:

(1) $98 \%$, Aldrich Chemical Company, Milwaukee, WI, USA, recrystallized three times from anhydrous methanol.

(2) $99.9+\%$, HPLC grade, Aldrich Chemical Company.

(3) $99+\%$, anhydrous, Aldrich Chemical Company.

(4) $99+\%$, anhydrous, Aldrich Chemical Company.

Components 2-4 were stored over molecular sieves and distilled shortly before use.

Estimated Error:

Temperature: $\pm 0.1 \mathrm{~K}$

$x_{3}{ }^{(\mathrm{s})}: \pm 0.0001$

$x_{4}{ }^{(\mathrm{s})}: \pm 0.0001$

$x_{1}: \pm 1.5 \%$ (relative error)

\begin{tabular}{ll}
\hline \hline Components: & Original Measurements: \\
(1) Pyrene; $\mathrm{C}_{16} \mathrm{H}_{10} ;[129-00-0]$ & ${ }^{32}$ E. M. Debase and W. E. Acree, \\
(2) Cyclohexane; $\mathrm{C}_{6} \mathrm{H}_{12} ;[110-82-7]$ & Jr., J. Chem. Eng. Data 46, 991 \\
(3) 2-Propanol; $\mathrm{C}_{3} \mathrm{H}_{8} \mathrm{O} ;[67-63-0]$ & $(2001)$. \\
(4) 1-Butanol; $\mathrm{C}_{4} \mathrm{H}_{10} \mathrm{O} ;[71-36-3]$ & \\
\hline Variables: & Prepared by: \\
$T / \mathrm{K}=299.15 ;$ Solvent Composition & W. E. Acree, Jr. \\
\hline
\end{tabular}

\section{Experimental Values}

Solubility of pyrene in cyclohexane +2 -propanol +1 -butanol mixtures ${ }^{\mathrm{a}}$

\begin{tabular}{lcccc}
\hline \hline$x_{3}{ }^{(\mathrm{s})}$ & $x_{4}{ }^{(\mathrm{s})}$ & $x_{3}$ & $x_{4}$ & $x_{1}$ \\
\hline 0.0000 & 0.0000 & 0.0000 & 0.0000 & 0.01100 \\
1.0000 & 0.0000 & 0.9971 & 0.0000 & 0.00290 \\
0.0000 & 1.0000 & 0.0000 & 0.9938 & 0.00622 \\
0.4549 & 0.2995 & 0.4718 & 0.2975 & 0.00684 \\
0.1788 & 0.7010 & 0.1776 & 0.6963 & 0.00669 \\
0.3076 & 0.2679 & 0.3049 & 0.2655 & 0.00887 \\
0.2958 & 0.4970 & 0.2937 & 0.4935 & 0.00696 \\
0.7460 & 0.0979 & 0.7423 & 0.0974 & 0.00495 \\
0.7437 & 0.1814 & 0.7405 & 0.1806 & 0.00429 \\
0.2443 & 0.6615 & 0.2428 & 0.6574 & 0.00617 \\
0.1300 & 0.5116 & 0.1288 & 0.5070 & 0.00898 \\
0.4572 & 0.4615 & 0.4547 & 0.4591 & 0.00530 \\
0.5517 & 0.3664 & 0.5490 & 0.3646 & 0.00499 \\
0.1786 & 0.1734 & 0.1767 & 0.1715 & 0.01082 \\
0.7434 & 0.1399 & 0.7399 & 0.1392 & 0.00473 \\
0.5627 & 0.2380 & 0.5593 & 0.2366 & 0.00603 \\
0.1376 & 0.2218 & 0.1361 & 0.2194 & 0.01101 \\
0.1316 & 0.7029 & 0.1307 & 0.6779 & 0.00715 \\
0.2681 & 0.1042 & 0.2653 & 0.1031 & 0.01056 \\
0.1360 & 0.4241 & 0.1347 & 0.4200 & 0.00965 \\
0.4748 & 0.1071 & 0.4708 & 0.1062 & 0.00835 \\
0.5665 & 0.1045 & 0.5624 & 0.1037 & 0.00727 \\
\hline$x_{3} \mathrm{~s}:$ & 0.1675
\end{tabular}

${ }^{\mathrm{a}} x_{3}{ }^{(\mathrm{s})}$ : initial mole fraction of component 3 in the ternary solvent mixture, $x_{4}{ }^{(\mathrm{s})}$ : initial mole fraction of component 4 in the ternary solvent mixture; $x_{1}$ : mole fraction solubility of the solute; $x_{3}$ : mole fraction of component 3 in the quaternary solution; $x_{4}$ : mole fraction of component 4 in the quaternary solution.

\section{Auxiliary Information}

\section{Method/Apparatus/Procedure:}

Constant-temperature bath, calorimetric thermometer, and an ultraviolet/ visible spectrophotometer.
Ternary solvent mixtures were prepared by mass. Excess solute and solvent were placed in amber glass bottles and allowed to equilibrate for several days at constant temperature. Attainment of equilibrium was verified by several repetitive measurements and by approaching equilibrium from supersaturation. Aliquots of saturated solutions were transferred through a coarse filter into volumetric flasks, weighed and diluted with methanol. Molar concentrations were determined by spectrophotometric measurements at $372 \mathrm{~nm}$.

\section{Source and Purity of Chemicals:}

(1) $98 \%$, Aldrich Chemical Company, Milwaukee, WI, USA, recrystallized three times from anhydrous methanol.

(2) $99.9+\%$, HPLC grade, Aldrich Chemical Company.

(3) $99+\%$, anhydrous, Aldrich Chemical Company.

(4) $99+\%$, anhydrous, Aldrich Chemical Company.

Components 2-4 were stored over molecular sieves and distilled shortly before use.

\section{Estimated Error:}

Temperature: $\pm 0.1 \mathrm{~K}$.

$x_{3}{ }^{(s)}: \pm 0.0001$.

$x_{4}{ }^{(\mathrm{s})}: \pm 0.0001$.

$x_{1}: \pm 1.5 \%$ (relative error).

\begin{tabular}{ll}
\hline \hline Components: & Original Measurements: \\
(1) Pyrene; $\mathrm{C}_{16} \mathrm{H}_{10} ;[129-00-0]$ & ${ }^{32}$ E. M. Debase and W. E. Acree, \\
(2) Cyclohexane; $\mathrm{C}_{6} \mathrm{H}_{12} ;[110-82-7]$ & Jr., J. Chem. Eng. Data 46, 991 \\
(3) 1-Propanol; $\mathrm{C}_{3} \mathrm{H}_{8} \mathrm{O} ;[71-23-8]$ & $(2001)$. \\
(4) 2-Butanol; $\mathrm{C}_{4} \mathrm{H}_{10} \mathrm{O} ;[78-92-2]$ &
\end{tabular}

\begin{tabular}{ll}
\hline Variables: & Prepared by: \\
$T / \mathrm{K}=299.15 ;$ Solvent Composition & W. E. Acree, Jr. \\
\hline
\end{tabular}

Experimental Values

Solubility of pyrene in cyclohexane +1 -propanol +2 -butanol mixtures ${ }^{\mathrm{a}}$

\begin{tabular}{|c|c|c|c|c|}
\hline$x_{3}{ }^{(s)}$ & $x_{4}^{(\mathrm{s})}$ & $x_{3}$ & $x_{4}$ & $x_{1}$ \\
\hline 0.0000 & 0.0000 & 0.0000 & 0.0000 & 0.01100 \\
\hline 1.0000 & 0.0000 & 0.9957 & 0.0000 & 0.00426 \\
\hline 0.0000 & 1.0000 & 0.0000 & 0.9956 & 0.00439 \\
\hline 0.4037 & 0.3266 & 0.4007 & 0.3242 & 0.00733 \\
\hline 0.1889 & 0.6895 & 0.1878 & 0.6856 & 0.00565 \\
\hline 0.3144 & 0.2626 & 0.3116 & 0.2602 & 0.00900 \\
\hline 0.3088 & 0.4762 & 0.3067 & 0.4730 & 0.00667 \\
\hline 0.7493 & 0.0941 & 0.7447 & 0.0935 & 0.00608 \\
\hline 0.7448 & 0.1788 & 0.7409 & 0.1779 & 0.00517 \\
\hline 0.2384 & 0.6688 & 0.2371 & 0.6653 & 0.00529 \\
\hline 0.1330 & 0.5069 & 0.1319 & 0.5026 & 0.00849 \\
\hline 0.4606 & 0.4567 & 0.4582 & 0.4543 & 0.00521 \\
\hline 0.5526 & 0.3608 & 0.5497 & 0.3589 & 0.00517 \\
\hline 0.2072 & 0.1706 & 0.2050 & 0.1688 & 0.01080 \\
\hline 0.7413 & 0.1413 & 0.7371 & 0.1406 & 0.00560 \\
\hline 0.5650 & 0.2337 & 0.5613 & 0.2322 & 0.00648 \\
\hline 0.1479 & 0.2207 & 0.1463 & 0.2183 & 0.01087 \\
\hline 0.1338 & 0.6917 & 0.1330 & 0.6873 & 0.00634 \\
\hline 0.2636 & 0.1214 & 0.2608 & 0.1201 & 0.01064 \\
\hline 0.1357 & 0.4208 & 0.1344 & 0.4169 & 0.00933 \\
\hline 0.4769 & 0.1107 & 0.4726 & 0.1097 & 0.00897 \\
\hline$\underline{0.5716}$ & 0.1049 & 0.5670 & 0.1041 & 0.00797 \\
\hline \multicolumn{5}{|c|}{$\begin{array}{l}{ }^{a} x_{3}{ }^{(\mathrm{s})} \text { : initial mole fraction of component } 3 \text { in the ternary solvent mixture, } x_{4}{ }^{\mathrm{s}} \\
\text { initial mole fraction of component } 4 \text { in the ternary solvent mixture; } x_{1}: \text { mol } \\
\text { fraction solubility of the solute; } x_{3} \text { : mole fraction of component } 3 \text { in the } \\
\text { quaternary solution; } x_{4} \text { : mole fraction of component } 4 \text { in the quaternary } \\
\text { solution. }\end{array}$} \\
\hline
\end{tabular}




\section{Auxiliary Information}

Method/Apparatus/Procedure:

Constant-temperature bath, calorimetric thermometer, and an ultraviolet/ visible spectrophotometer.

Ternary solvent mixtures were prepared by mass. Excess solute and solvent were placed in amber glass bottles and allowed to equilibrate for several days at constant temperature. Attainment of equilibrium was verified by several repetitive measurements and by approaching equilibrium from supersaturation. Aliquots of saturated solutions were transferred through a coarse filter into volumetric flasks, weighed and diluted with methanol. Molar concentrations were determined by spectrophotometric measurements at $372 \mathrm{~nm}$.

\section{Source and Purity of Chemicals:}

(1) $98 \%$, Aldrich Chemical Company, Milwaukee, WI, USA, recrystallized three times from anhydrous methanol.

(2) $99.9+\%$, HPLC grade, Aldrich Chemical Company.

(3) $99+\%$, anhydrous, Aldrich Chemical Company.

(4) $99+\%$, anhydrous, Aldrich Chemical Company.

Components 2-4 were stored over molecular sieves and distilled shortly before use.

\section{Estimated Error:}

Temperature: $\pm 0.1 \mathrm{~K}$.

$x_{3}{ }^{(\mathrm{s})}: \pm 0.0001$.

$x_{4}{ }^{(\mathrm{s})}: \pm 0.0001$.

$x_{1}: \pm 1.5 \%$ (relative error).

\section{Components:}

(1) Pyrene; $\mathrm{C}_{16} \mathrm{H}_{10}$; [129-00-0]

(2) Cyclohexane; $\mathrm{C}_{6} \mathrm{H}_{12} ;$ [110-82-7]

(3) 2-Propanol; $\mathrm{C}_{3} \mathrm{H}_{8} \mathrm{O}$; [67-63-0]

(4) 2-Butanol; $\mathrm{C}_{4} \mathrm{H}_{10} \mathrm{O}$; [78-92-2]

\section{Original Measurements:}

${ }^{32}$ E. M. Debase and W. E. Acree,

Jr., J. Chem. Eng. Data 46, 991

(2001).

\begin{tabular}{ll}
\hline Variables: & Prepared by: \\
$T / \mathrm{K}=299.15 ;$ Solvent Composition & W. E. Acree, Jr. \\
\hline
\end{tabular}

Experimental Values

Solubility of pyrene in cyclohexane +2 -propanol +2 -butanol mixtures ${ }^{\mathrm{a}}$

\begin{tabular}{lcccc}
\hline \hline$x_{3}{ }^{(\mathrm{s})}$ & $x_{4}{ }^{(\mathrm{s})}$ & $x_{3}$ & $x_{4}$ & $x_{1}$ \\
\hline 0.0000 & 0.0000 & 0.0000 & 0.0000 & 0.01100 \\
1.0000 & 0.0000 & 0.9971 & 0.0000 & 0.00290 \\
0.0000 & 1.0000 & 0.0000 & 0.9956 & 0.00439 \\
0.3935 & 0.3290 & 0.3908 & 0.3268 & 0.00682 \\
0.1841 & 0.6901 & 0.1831 & 0.6863 & 0.00556 \\
0.3142 & 0.2591 & 0.3115 & 0.2568 & 0.00873 \\
0.3008 & 0.4867 & 0.2989 & 0.4836 & 0.00632 \\
0.7502 & 0.0973 & 0.7465 & 0.0968 & 0.00488 \\
0.7393 & 0.1797 & 0.7362 & 0.1789 & 0.00420 \\
0.2415 & 0.6756 & 0.2403 & 0.6722 & 0.00498 \\
0.1350 & 0.5138 & 0.1339 & 0.5096 & 0.00821 \\
0.4476 & 0.4691 & 0.4455 & 0.4669 & 0.00460 \\
0.5398 & 0.3687 & 0.5373 & 0.3670 & 0.00458 \\
0.1975 & 0.1683 & 0.1954 & 0.1665 & 0.01058 \\
0.7449 & 0.1408 & 0.7416 & 0.1402 & 0.00443 \\
0.5560 & 0.2438 & 0.5528 & 0.2424 & 0.00570
\end{tabular}

0.2438

0.5528

0.2424

$\begin{array}{lllll}0.1389 & 0.2273 & 0.1374 & 0.2249 & 0.01071 \\ 0.1324 & 0.7012 & 0.1316 & 0.6970 & 0.00603 \\ 0.2614 & 0.1141 & 0.2587 & 0.1129 & 0.01048 \\ 0.1380 & 0.4184 & 0.1367 & 0.4146 & 0.00913 \\ 0.4650 & 0.1198 & 0.4612 & 0.1188 & 0.00823 \\ 0.5674 & 0.1067 & 0.5634 & 0.1060 & 0.00698\end{array}$

${ }^{\mathrm{a}} x_{3}{ }^{(\mathrm{s})}$ : initial mole fraction of component 3 in the ternary solvent mixture, $x_{4}{ }^{(\mathrm{s})}$ : initial mole fraction of component 4 in the ternary solvent mixture; $x_{1}$ : mole fraction solubility of the solute; $x_{3}$ : mole fraction of component 3 in the quaternary solution; $x_{4}$ : mole fraction of component 4 in the quaternary solution.
Method/Apparatus/Procedure:

Constant-temperature bath, calorimetric thermometer, and an ultraviolet/ visible spectrophotometer.

Ternary solvent mixtures were prepared by mass. Excess solute and solvent were placed in amber glass bottles and allowed to equilibrate for several days at constant temperature. Attainment of equilibrium was verified by several repetitive measurements and by approaching equilibrium from supersaturation. Aliquots of saturated solutions were transferred through a coarse filter into volumetric flasks, weighed and diluted with methanol. Molar concentrations were determined by spectrophotometric measurements at $372 \mathrm{~nm}$.

\section{Source and Purity of Chemicals:}

(1) $98 \%$, Aldrich Chemical Company, Milwaukee, WI, USA, recrystallized three times from anhydrous methanol.

(2) $99.9+\%$, HPLC grade, Aldrich Chemical Company.

(3) $99+\%$, anhydrous, Aldrich Chemical Company.

(4) $99+\%$, anhydrous, Aldrich Chemical Company.

Components 2-4 were stored over molecular sieves and distilled shortly before use.

Estimated Error:

Temperature: $\pm 0.1 \mathrm{~K}$.

$x_{3}{ }^{(\mathrm{s})}: \pm 0.0001$.

$x_{4}{ }^{(\mathrm{s})}: \pm 0.0001$.

$x_{1}: \pm 1.5 \%$ (relative error)

\subsection{Pyrene solubility data in ternary $2,2,4-$ trimethylpentane + alcohol + alcohol solvent mixtures}

\section{Components:}

(1) Pyrene; $\mathrm{C}_{16} \mathrm{H}_{10}$; [129-00-0]

(2) 2,2,4-Trimethylpentane; $\mathrm{C}_{8} \mathrm{H}_{18}$;

[540-84-1]

(3) 1-Propanol; $\mathrm{C}_{3} \mathrm{H}_{8} \mathrm{O}$; [71-23-8]

(4) 1-Butanol; $\mathrm{C}_{4} \mathrm{H}_{10} \mathrm{O}$; [71-36-3]

\begin{tabular}{ll}
\hline Variables: & Prepared by: \\
$T / \mathrm{K}=299.15 ;$ Solvent Composition & W. E. Acree, Jr. \\
\hline
\end{tabular}

Original Measurements:

${ }^{30}$ E. M. Debase and W. E. Acree, Jr., J. Chem. Eng. Data 46, 1464 (2001). 


\section{Experimental Values}

Solubility of pyrene in 2,2,4-trimethylpentane +1 -propanol +1 -butanol mixtures $^{\mathrm{a}}$

\begin{tabular}{lcccc}
\hline \hline$x_{3}{ }^{(\mathrm{s})}$ & $x_{4}{ }^{(\mathrm{s})}$ & $x_{3}$ & $x_{4}$ & $x_{1}$ \\
\hline 0.0000 & 0.0000 & 0.0000 & 0.0000 & 0.00720 \\
1.0000 & 0.0000 & 0.9957 & 0.0000 & 0.00426 \\
0.0000 & 1.0000 & 0.0000 & 0.9938 & 0.00622 \\
0.4337 & 0.3632 & 0.4308 & 0.3608 & 0.00661 \\
0.1954 & 0.7132 & 0.1941 & 0.7085 & 0.00653 \\
0.3581 & 0.2965 & 0.3556 & 0.2944 & 0.00709 \\
0.3244 & 0.5305 & 0.3223 & 0.5270 & 0.00661 \\
0.7957 & 0.1003 & 0.7914 & 0.0998 & 0.00536 \\
0.7661 & 0.1791 & 0.7621 & 0.1782 & 0.00517 \\
0.2455 & 0.6917 & 0.2439 & 0.6873 & 0.00638 \\
0.1611 & 0.5824 & 0.1599 & 0.5781 & 0.00732 \\
0.4596 & 0.4840 & 0.4569 & 0.4812 & 0.00580 \\
0.5712 & 0.3729 & 0.5681 & 0.3708 & 0.00551 \\
0.2562 & 0.2094 & 0.2542 & 0.2078 & 0.00781 \\
0.7831 & 0.1395 & 0.7790 & 0.1388 & 0.00528 \\
0.6042 & 0.2581 & 0.6007 & 0.2566 & 0.00584 \\
0.1896 & 0.2805 & 0.1881 & 0.2783 & 0.00792 \\
01432 & 0.7376 & 0.1422 & 0.7326 & 0.00684 \\
0.3384 & 0.1481 & 0.3358 & 0.1470 & 0.00758 \\
0.1596 & 0.4958 & 0.1584 & 0.4920 & 0.00770 \\
0.5475 & 0.1267 & 0.5439 & 0.1259 & 0.00653 \\
0.6513 & 0.1161 & 0.6473 & 0.1154 & 0.00616 \\
\hline $\mathrm{a}_{x_{3}{ }^{(\mathrm{s})} \text {. initial mole fraction }}$ & &
\end{tabular}

: initial mole fraction of component 3 in the ternary solvent mixture, $x_{4}{ }^{(\mathrm{s})}$ initial mole fraction of component 4 in the ternary solvent mixture; $x_{1}$ : mole fraction solubility of the solute; $x_{3}$ : mole fraction of component 3 in the quaternary solution; $x_{4}$ : mole fraction of component 4 in the quaternary solution.

\section{Auxiliary Information}

\section{Method/Apparatus/Procedure:}

Constant-temperature bath, calorimetric thermometer, and an ultraviolet/ visible spectrophotometer.

Ternary solvent mixtures were prepared by mass. Excess solute and solvent were placed in amber glass bottles and allowed to equilibrate for several days at constant temperature. Attainment of equilibrium was verified by several repetitive measurements and by approaching equilibrium from supersaturation. Aliquots of saturated solutions were transferred through a coarse filter into volumetric flasks, weighed and diluted with methanol. Molar concentrations were determined by spectrophotometric measurements at $372 \mathrm{~nm}$.

\section{Source and Purity of Chemicals:}

(1) $98 \%$, Aldrich Chemical Company, Milwaukee, WI, USA, recrystallized three times from anhydrous methanol.

(2) $99.8 \%$, anhydrous, Aldrich Chemical Company.

(3) $99+\%$, anhydrous, Aldrich Chemical Company.

(4) $99+\%$, anhydrous, Aldrich Chemical Company.

Components $2-4$ were stored over molecular sieves and distilled shortly before use.

\section{Estimated Error:}

Temperature: $\pm 0.1 \mathrm{~K}$

$x_{3}^{(\mathrm{s})}: \pm 0.0001$.

$x_{4}{ }^{(\mathrm{s})}: \pm 0.0001$.

$x_{1}: \pm 1.5 \%$ (relative error)

\begin{tabular}{|c|c|}
\hline $\begin{array}{l}\text { Components: } \\
\text { (1) Pyrene; } \mathrm{C}_{16} \mathrm{H}_{10} ;[129-00-0] \\
\text { (2) 2,2,4-Trimethylpentane; } \mathrm{C}_{8} \mathrm{H}_{18} \text {; } \\
\text { [540-84-1] } \\
\text { (3) 2-Propanol; } \mathrm{C}_{3} \mathrm{H}_{8} \mathrm{O} ;[67-63-0] \\
\text { (4) 1-Butanol; } \mathrm{C}_{4} \mathrm{H}_{10} \mathrm{O} ;[71-36-3]\end{array}$ & $\begin{array}{l}\text { Original Measurements: } \\
{ }^{30} \text { E. M. Debase and W. E. Acree, } \\
\text { Jr., J. Chem. Eng. Data 46, } 1464 \\
(2001) \text {. }\end{array}$ \\
\hline $\begin{array}{l}\text { Variables: } \\
T / \mathrm{K}=299.15 ; \text { Solvent Composition }\end{array}$ & $\begin{array}{l}\text { Prepared by: } \\
\text { W. E. Acree, Jr. }\end{array}$ \\
\hline
\end{tabular}

Experimental Values

Solubility of pyrene in 2,2,4-trimethylpentane +2 -propanol + 1-butanol mixtures $^{\mathrm{a}}$

\begin{tabular}{lcccc}
\hline \hline$x_{3}^{(\mathrm{s})}$ & $x_{4}^{(\mathrm{s})}$ & $x_{3}$ & $x_{4}$ & $x_{1}$ \\
\hline 0.0000 & 0.0000 & 0.0000 & 0.0000 & 0.00720 \\
1.0000 & 0.0000 & 0.9971 & 0.0000 & 0.00290 \\
0.0000 & 1.0000 & 0.0000 & 0.9938 & 0.00622 \\
0.3918 & 0.3835 & 0.3895 & 0.3812 & 0.00596 \\
0.1896 & 0.7216 & 0.1884 & 0.7171 & 0.00627 \\
0.3640 & 0.3114 & 0.3616 & 0.3094 & 0.00654 \\
0.3236 & 0.5234 & 0.3216 & 0.5202 & 0.00606 \\
0.7981 & 0.0970 & 0.7948 & 0.0966 & 0.00419 \\
0.7632 & 0.1826 & 0.7601 & 0.1819 & 0.00405 \\
0.2404 & 0.7010 & 0.2390 & 0.6969 & 0.00590 \\
0.1507 & 0.5985 & 0.1496 & 0.5943 & 0.00699 \\
0.4519 & 0.4870 & 0.4496 & 0.4845 & 0.00507 \\
0.5603 & 0.3834 & 0.5577 & 0.3816 & 0.00458 \\
0.2550 & 0.2061 & 0.2531 & 0.2046 & 0.00748 \\
0.7777 & 0.1382 & 0.7745 & 0.1376 & 0.00414 \\
0.6063 & 0.2474 & 0.6033 & 0.2462 & 0.00498 \\
0.1768 & 0.2812 & 0.1754 & 0.2790 & 0.00771 \\
0.1364 & 0.7433 & 0.1355 & 0.7385 & 0.00650 \\
0.3285 & 0.1478 & 0.3261 & 0.1467 & 0.00716 \\
0.1608 & 0.4971 & 0.1596 & 0.4935 & 0.00730 \\
0.5479 & 0.1281 & 0.5447 & 0.1273 & 0.00592 \\
0.6421 & 0.1195 & 0.6388 & 0.1189 & 0.00521 \\
\hline
\end{tabular}

${ }^{\mathrm{a}} x_{3}{ }^{(\mathrm{s})}$ : initial mole fraction of component 3 in the ternary solvent mixture, $x_{4}{ }^{(\mathrm{s})}$ : initial mole fraction of component 4 in the ternary solvent mixture; $x_{1}$ : mole fraction solubility of the solute; $x_{3}$ : mole fraction of component 3 in the quaternary solution; $x_{4}$ : mole fraction of component 4 in the quaternary solution.

\section{Auxiliary Information}

\section{Method/Apparatus/Procedure:}

Constant-temperature bath, calorimetric thermometer, and an ultraviolet/ visible spectrophotometer.

Ternary solvent mixtures were prepared by mass. Excess solute and solvent were placed in amber glass bottles and allowed to equilibrate for several days at constant temperature. Attainment of equilibrium was verified by several repetitive measurements and by approaching equilibrium from supersaturation. Aliquots of saturated solutions were transferred through a coarse filter into volumetric flasks, weighed and diluted with methanol. Molar concentrations were determined by spectrophotometric measurements at $372 \mathrm{~nm}$.

\section{Source and Purity of Chemicals:}

(1) $98 \%$, Aldrich Chemical Company, Milwaukee, WI, USA, recrystallized three times from anhydrous methanol.

(2) $99.8 \%$, anhydrous, Aldrich Chemical Company.

(3) $99+\%$, anhydrous, Aldrich Chemical Company.

(4) $99+\%$, anhydrous, Aldrich Chemical Company.

Components 2-4 were stored over molecular sieves and distilled shortly before use. 
Estimated Error:

Temperature: $\pm 0.1 \mathrm{~K}$.

$x_{3}{ }^{(\mathrm{s})}: \pm 0.0001$.

$x_{4}{ }^{(\mathrm{s})}: \pm 0.0001$.

$x_{1}: \pm 1.5 \%$ (relative error).

\begin{tabular}{|c|c|}
\hline $\begin{array}{l}\text { Components: } \\
\text { (1) Pyrene; } \mathrm{C}_{16} \mathrm{H}_{10} ;[129-00-0] \\
\text { (2) 2,2,4-Trimethylpentane; } \mathrm{C}_{8} \mathrm{H}_{18} \text {; } \\
\text { [540-84-1] } \\
\text { (3) 1-Propanol; } \mathrm{C}_{3} \mathrm{H}_{8} \mathrm{O} ;[71-23-8] \\
\text { (4) 2-Butanol; } \mathrm{C}_{4} \mathrm{H}_{10} \mathrm{O} ;[78-92-2]\end{array}$ & $\begin{array}{l}\text { Original Measurements: } \\
{ }^{30} \text { E. M. Debase and W. E. Acree, } \\
\text { Jr., J. Chem. Eng. Data 46, } 1464 \\
(2001) \text {. }\end{array}$ \\
\hline $\begin{array}{l}\text { Variables: } \\
T / \mathrm{K}=299.15 ; \text { Solvent Composition }\end{array}$ & $\begin{array}{l}\text { Prepared by: } \\
\text { W. E. Acree, Jr. }\end{array}$ \\
\hline
\end{tabular}

Experimental Values

Solubility of pyrene in 2,2,4-trimethylpentane+ 1-propanol + 2-butanol mixtures $^{\mathrm{a}}$

\begin{tabular}{|c|c|c|c|c|}
\hline$x_{3}{ }^{(\mathrm{s})}$ & $x_{4}{ }^{(\mathrm{s})}$ & $x_{3}$ & $x_{4}$ & $x_{1}$ \\
\hline 0.0000 & 0.0000 & 0.0000 & 0.0000 & 0.00720 \\
\hline 1.0000 & 0.0000 & 0.9957 & 0.0000 & 0.00426 \\
\hline 0.0000 & 1.0000 & 0.0000 & 0.9956 & 0.00439 \\
\hline 0.4351 & 0.3621 & 0.4326 & 0.3600 & 0.00581 \\
\hline 0.2009 & 0.7124 & 0.1999 & 0.7088 & 0.00511 \\
\hline 0.3685 & 0.3007 & 0.3661 & 0.2987 & 0.00650 \\
\hline 0.3263 & 0.5201 & 0.3245 & 0.5173 & 0.00545 \\
\hline 0.7961 & 0.0993 & 0.7922 & 0.0988 & 0.00495 \\
\hline 0.7673 & 0.1830 & 0.7637 & 0.1821 & 0.00471 \\
\hline 0.2508 & 0.6924 & 0.2496 & 0.6890 & 0.00493 \\
\hline 0.1544 & 0.5877 & 0.1534 & 0.5839 & 0.00639 \\
\hline 0.4716 & 0.4710 & 0.4693 & 0.4687 & 0.00482 \\
\hline 0.5737 & 0.3703 & 0.5710 & 0.3685 & 0.00479 \\
\hline 0.2627 & 0.2108 & 0.2608 & 0.2092 & 0.00738 \\
\hline 0.7883 & 0.1359 & 0.7844 & 0.1352 & 0.00492 \\
\hline 0.6038 & 0.2523 & 0.6005 & 0.2509 & 0.00544 \\
\hline 0.1756 & 0.2783 & 0.1743 & 0.2762 & 0.00750 \\
\hline 0.1372 & 0.7424 & 0.1365 & 0.7384 & 0.00541 \\
\hline 0.3489 & 0.1292 & 0.3864 & 0.1283 & 0.00725 \\
\hline 0.1669 & 0.4869 & 0.1658 & 0.4836 & 0.00671 \\
\hline 0.5649 & 0.1134 & 0.5613 & 0.1127 & 0.00640 \\
\hline 0.6598 & 0.1033 & 0.6559 & 0.1027 & 0.00593 \\
\hline
\end{tabular}

${ }^{\mathrm{a}} x_{3}{ }^{(\mathrm{s})}$ : initial mole fraction of component 3 in the ternary solvent mixture, $x_{4}{ }^{(\mathrm{s})}$ : initial mole fraction of component 4 in the ternary solvent mixture; $x_{1}$ : mole fraction solubility of the solute; $x_{3}$ : mole fraction of component 3 in the quaternary solution; $x_{4}$ : mole fraction of component 4 in the quaternary solution.

\section{Auxiliary Information}

\section{Method/Apparatus/Procedure:}

Constant-temperature bath, calorimetric thermometer, and an ultraviolet/ visible spectrophotometer.

Ternary solvent mixtures were prepared by mass. Excess solute and solvent were placed in amber glass bottles and allowed to equilibrate for several days at constant temperature. Attainment of equilibrium was verified by several repetitive measurements and by approaching equilibrium from supersaturation. Aliquots of saturated solutions were transferred through a coarse filter into volumetric flasks, weighed and diluted with methanol. Molar concentrations were determined by spectrophotometric measurements at $372 \mathrm{~nm}$.

Source and Purity of Chemicals:

(1) $98 \%$, Aldrich Chemical Company, Milwaukee, WI, USA, recrystallized three times from anhydrous methanol.

(2) $99.8 \%$, anhydrous, Aldrich Chemical Company.

(3) $99+\%$, anhydrous, Aldrich Chemical Company.

(4) $99+\%$, anhydrous, Aldrich Chemical Company.

Components 2-4 were stored over molecular sieves and distilled shortly before use.

\section{Estimated Error:}

Temperature: $\pm 0.1 \mathrm{~K}$.

$x_{3}{ }^{(s)}: \pm 0.0001$.

$x_{4}{ }^{(\mathrm{s})}: \pm 0.0001$.

$x_{1}: \pm 1.5 \%$ (relative error).

\section{Components:}

(1) Pyrene; $\mathrm{C}_{16} \mathrm{H}_{10} ;$ [129-00-0]

(2) 2,2,4-Trimethylpentane; $\mathrm{C}_{8} \mathrm{H}_{18}$; [540-84-1]

(3) 2-Propanol; $\mathrm{C}_{3} \mathrm{H}_{8} \mathrm{O}$; [67-63-0]

(4) 2-Butanol; $\mathrm{C}_{4} \mathrm{H}_{10} \mathrm{O}$; [78-92-2]

\begin{tabular}{ll}
\hline Variables: & Prepared by: \\
$T / \mathrm{K}=299.15$; Solvent Composition & W. E. Acree, Jr. \\
\hline
\end{tabular}

Experimental Values

Solubility of pyrene in 2,2,4-trimethylpentane +2 -propanol + 2-butanol mixtures $^{\mathrm{a}}$

\begin{tabular}{lcccc}
\hline \hline$x_{3}(\mathrm{~s})$ & $x_{4}{ }^{(\mathrm{s})}$ & $x_{3}$ & $x_{4}$ & $x_{1}$ \\
\hline 0.0000 & 0.0000 & 0.0000 & 0.0000 & 0.00720 \\
1.0000 & 0.0000 & 0.9971 & 0.0000 & 0.00290 \\
0.0000 & 1.0000 & 0.0000 & 0.9956 & 0.00439 \\
0.4306 & 0.3642 & 0.4283 & 0.3623 & 0.00523 \\
0.1915 & 0.7210 & 0.1906 & 0.7175 & 0.00483 \\
0.3719 & 0.2972 & 0.3697 & 0.2954 & 0.00597 \\
0.3282 & 0.5236 & 0.3265 & 0.5210 & 0.00504 \\
0.7910 & 0.1024 & 0.7878 & 0.1020 & 0.00402 \\
0.7705 & 0.1780 & 0.7677 & 0.1773 & 0.00367 \\
0.2433 & 0.7056 & 0.2422 & 0.7025 & 0.00442 \\
0.1640 & 0.5736 & 0.1630 & 0.5701 & 0.00610 \\
0.4714 & 0.4747 & 0.4695 & 0.4727 & 0.00409 \\
0.5725 & 0.3750 & 0.5703 & 0.3735 & 0.00390 \\
0.2544 & 0.2113 & 0.2526 & 0.2098 & 0.00704 \\
0.7782 & 0.1440 & 0.7752 & 0.1434 & 0.00387 \\
0.6006 & 0.2592 & 0.5978 & 0.2580 & 0.00464 \\
0.1811 & 0.2819 & 0.1798 & 0.2799 & 0.00714 \\
0.1420 & 0.7372 & 0.1413 & 0.7334 & 0.00511 \\
0.3332 & 0.1459 & 0.3309 & 0.1449 & 0.00680 \\
0.1607 & 0.4922 & 0.1596 & 0.4890 & 0.00657 \\
0.5584 & 0.1214 & 0.5553 & 0.1207 & 0.00549 \\
0.6505 & 0.1106 & 0.6472 & 0.1100 & 0.00504 \\
\hline$x_{3}(9)$ & &
\end{tabular}

${ }^{\mathrm{a}} x_{3}{ }^{(\mathrm{s})}$ : initial mole fraction of component 3 in the ternary solvent mixture, $x_{4}{ }^{(\mathrm{s})}$ : initial mole fraction of component 4 in the ternary solvent mixture; $x_{1}$ : mole fraction solubility of the solute; $x_{3}$ : mole fraction of component 3 in the quaternary solution; $x_{4}$ : mole fraction of component 4 in the quaternary solution. 


\section{Auxiliary Information}

\section{Method/Apparatus/Procedure:}

Constant-temperature bath, calorimetric thermometer, and an ultraviolet/ visible spectrophotometer.

Ternary solvent mixtures were prepared by mass. Excess solute and solvent were placed in amber glass bottles and allowed to equilibrate for several days at constant temperature. Attainment of equilibrium was verified by several repetitive measurements and by approaching equilibrium from supersaturation. Aliquots of saturated solutions were transferred through a coarse filter into volumetric flasks, weighed and diluted with methanol. Molar concentrations were determined by spectrophotometric measurements at $372 \mathrm{~nm}$.

\section{Source and Purity of Chemicals:}

(1) $98 \%$, Aldrich Chemical Company, Milwaukee, WI, USA, recrystallized three times from anhydrous methanol.

(2) $99.8 \%$, anhydrous, Aldrich Chemical Company.

(3) $99+\%$, anhydrous, Aldrich Chemical Company.

(4) $99+\%$, anhydrous, Aldrich Chemical Company.

Components 2-4 were stored over molecular sieves and distilled shortly before use.

\section{Estimated Error:}

Temperature: $\pm 0.1 \mathrm{~K}$

$x_{3}{ }^{(\mathrm{s})}: \pm 0.0001$

$x_{4}{ }^{(\mathrm{s})}: \pm 0.0001$

$x_{1}: \pm 1.5 \%$ (relative error)

\section{References}

${ }^{1}$ Polycyclic Aromatic Hydrocarbons in Pure and Binary Solvent Mixtures, edited by W. E. Acree, Jr., IUPAC Solubility Data Series Vol. 54 (Oxford University Press, London, 1994).

${ }^{2}$ Polycyclic Aromatic Hydrocarbons: Binary Nonaqueous Systems, Part I: Solutes A-E, edited by W. E. Acree, Jr., IUPAC Solubility Data Series Vol. 58 (Oxford University Press, London, 1995).

${ }^{3}$ Polycyclic Aromatic Hydrocarbons: Binary Nonaqueous Systems, Part 2. Solutes F-Z, edited by W. E. Acree, Jr., IUPAC Solubility Data Series Vol 59 (Oxford University Press, London, 1995).

${ }^{4}$ W. E. Acree, Jr., J. Phys. Chem. Ref. Data 42, 013103 (2013).

${ }^{5}$ W. E. Acree, Jr., and A. I. Zvaigzne, Thermochim. Acta 178, 151 (1991)

${ }^{6}$ W. E. Acree, Jr., J. W. McCargar, A. I. Zvaigzne, and I.-L. Teng, Phys. Chem. Liq. 23, 27 (1991).

${ }^{7}$ A. Proctor, B. H. Blake-Taylor, and W. E. Acree, Jr., J. Chem. Eng. Data 53, 2910 (2008).
${ }^{8}$ A. Proctor, B. A. Martine, and W. E. Acree, Jr., J. Chem. Eng. Data 53, 2197 (2008).

${ }^{9}$ B. H. Blake-Taylor, B. A. Martine, and W. E. Acree, Jr., J. Chem. Eng. Data 53, 970 (2008)

${ }^{10}$ A. Shayanfar, S. Soltani, F. Jabbaribar, E. Tamizi, W. E. Acree, Jr., and A. Jouyban, J. Chem. Eng. Data 53, 890 (2008).

${ }^{11}$ B. A. Martine, B. H. Blake-Taylor, and W. E. Acree, Jr., J. Chem. Eng. Data 53, 556 (2008)

${ }^{12}$ K. J. Pribyla, T. T. Van, C. Ezell, and W. E. Acree, Jr., J. Chem. Eng. Data 45, 968 (2000).

${ }^{13}$ K. J. Pribyla, M. A. Spurgin, I. Chuca, and W. E. Acree, Jr., J. Chem. Eng. Data 45, 965 (2000).

${ }^{14}$ K. J. Pribyla, M. A. Spurgin, I. Chuca, and W. E. Acree, Jr., J. Chem. Eng. Data 45, 971 (2000).

${ }^{15}$ K. J. Pribyla, C. Ezell, T. T. Van, and W. E. Acree, Jr., J. Chem. Eng. Data 45, 974 (2000).

${ }^{16}$ T. Deng, S. D. Childress, K. M. De Fina, and W. E. Acree, Jr., Chem. Eng. Commun. 172, 217 (1999).

${ }^{17}$ K. J. Pribyla, M. A. Spurgin, I. Chuca, and W. E. Acree, Jr., J. Chem. Eng. Data 45, 530 (2000).

${ }^{18}$ K. J. Pribyla, I. Chuca, T. T. Van, and W. E. Acree, Jr., J. Chem. Eng. Data 45, 533 (2000).

${ }^{19}$ K. J. Pribyla, M. A. Spurgin, I. Chuca, and W. E. Acree, Jr., J. Chem. Eng. Data 44, 1265 (1999).

${ }^{20}$ K. J. Pribyla and W. E. Acree, Jr., J. Chem. Eng. Data 44, 1259 (1999).

${ }^{21}$ K. J. Pribyla and W. E. Acree, Jr., J. Chem. Eng. Data 44, 1020 (1999).

${ }^{22}$ T. Deng and W. E. Acree, Jr., J. Chem. Eng. Data 44, 544 (1999).

${ }^{23}$ T. Deng, C. E. Hernandez, L. E. Roy, and W. E. Acree, Jr., J. Chem. Thermodyn. 31, 205 (1999).

${ }^{24}$ T. Deng, S. D. Childress, K. M. Fina, C. E. Hernandez, L. E. Roy, T. L. Sharp, and W. E. Acree, Jr., J. Chem. Eng. Data 44, 357 (1999).

${ }^{25}$ T. Deng, S. Horiuchi, L. E. Roy, and W. E. Acree, Jr., J. Chem. Eng. Data 44, 258 (1999).

${ }^{26}$ T. Deng, S. D. Childress, K. M. De Fina, T. L. Sharp, and W. E. Acree, Jr., J. Chem. Eng. Data 43, 1065 (1998).

${ }^{27}$ T. Deng and W. E. Acree, Jr., J. Chem. Eng. Data 43, 1059 (1998).

${ }^{28}$ T. Deng and W. E. Acree, Jr., J. Chem. Eng. Data 43, 1062 (1998).

${ }^{29}$ S. Fishback, S. Duenas, N. Kuehn, J. Pacheco, and W. E. Acree, Jr., J. Chem. Eng. Data 47, 62 (2002).

${ }^{30}$ E. M. Debase and W. E. Acree, Jr., J. Chem. Eng. Data 46, 1464 (2001).

${ }^{31}$ E. M. Debase and W. E. Acree, Jr., J. Chem. Eng. Data 46, 1297 (2001).

${ }^{32}$ E. M. Debase and W. E. Acree, Jr., J. Chem. Eng. Data 46, 991 (2001).

${ }^{33}$ A. Jouyban, J. L. Manzoori, V. Panahi-Azar, J. Soleymani, M. A. A. Fakhree, A. Shayanfar, and W. E. Acree, Jr., J. Chem. Eng. Data 55, 2607 (2010).

${ }^{34}$ M. A. A. Fakhree, W. E. Acree, Jr., and A. Jouyban, Ind. Eng. Chem. Res. 49, 6238 (2010). 